\title{
Effects of Surface Acoustic Waves in Ferromagnetic Nanostripes
}

\author{
By David Castilla Aragón
}

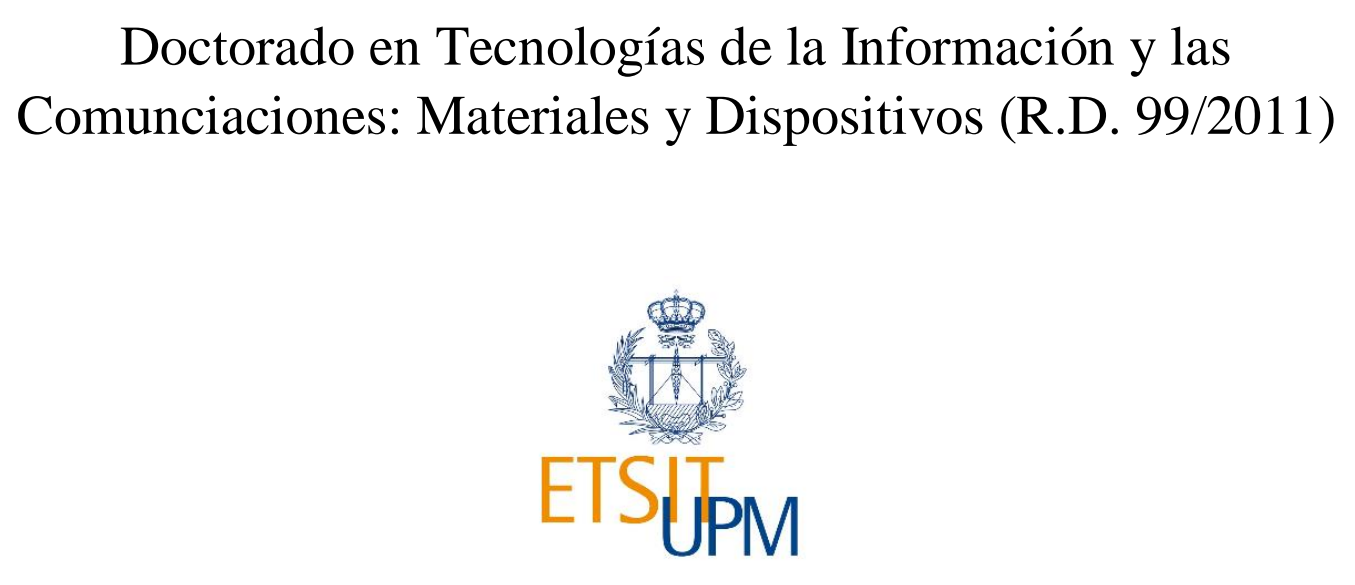

Advisors:

Dr. Jose Luis Prieto Martín

Dr. Manuel Muñoz Sánchez

Departamento de Física Electrónica

Escuela Técnica Superior de Ingenieros de Telecomunicación Universidad Politécnica de Madrid

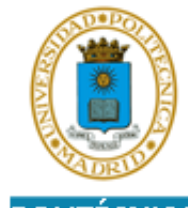


Title: Effects of Surface Acoustic waves on Ferromagnetic nanostripes 


\section{Abstract}

The study of magnetic nanostripes has gained importance in the last decade since this nanostructure is the basic element of proposed non-volatile memory devices. The achievement of the low power control of the magnetization states and the DW dynamics of the nanostripes would mean a considerable step forward for the technological development of these devices.

The magnetoelastic effect can be taken advantage of in order to assist the magnetization dynamics in the nanostripes, either in addition or instead of the most common method, the injection of a spin-polarized current. Surface acoustic waves (SAW's) are able to propagate strain in a periodic fashion and generate changes in the magnetic properties of the nanostructures. SAW's can induce an additional anisotropy in the direction of propagation of the waves as well as a decrease in the coercive fields of magnetic nanostructures.

The application of surface acoustic waves to magnetic nanostructure can also force the magnetic precession of the spins within the ferromagnets and the emission of a spin current in ferromagnetic/non-magnetic bilayers, in analogy to the mechanism of the ferromagnetic resonance. 


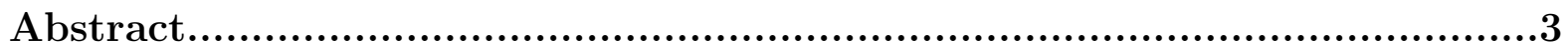

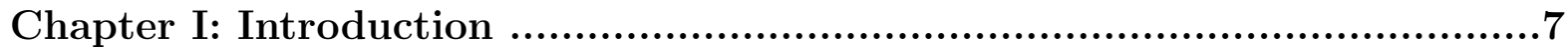

Chapter II: Experimental Techniques ..........................................15

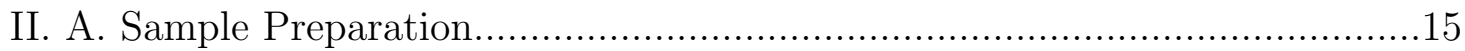

II. B. Structural Characterization............................................................. 22

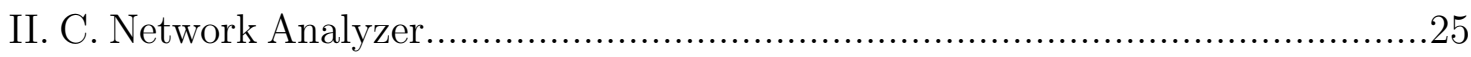

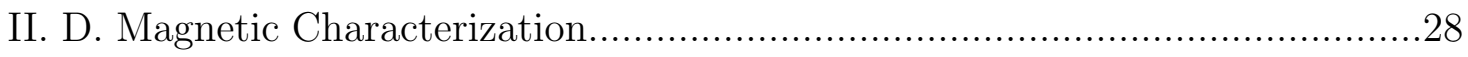

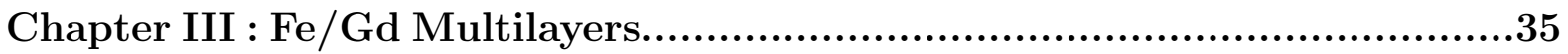

III. A. Ferromagnetic Resonance ….................................................. 36

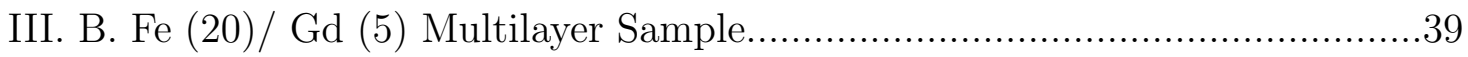

III. C. Fe (5)/ Gd (15) Multilayer Sample ......................................................42

III. D. Fe (10)/ Gd (10) Multilayer Sample...................................................... 45

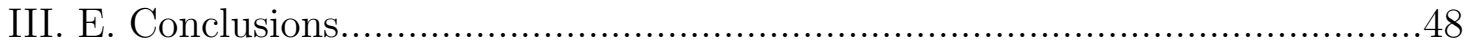

Chapter IV : Micromagnetic Simulations On Ni Nanowires.....................51

IV. A. Non-Magnetic Constraint ..............................................................52

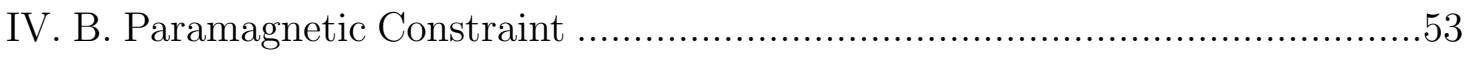

IV. C. Nucleating an Initial Transverse DW ..............................................55

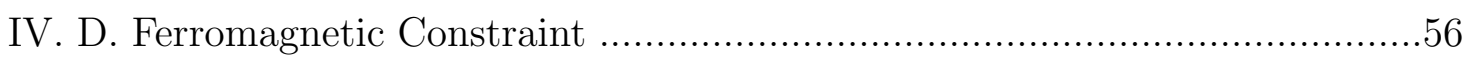

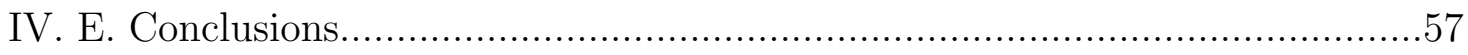

Chapter V : Structural Characterization.......................................59

V. A. Characterization of ScAlN Thin Films......................................................61

V. B. Characterization of Ni Thin Films...................................................64

Chapter VI : Magnetic Characterization...........................................73

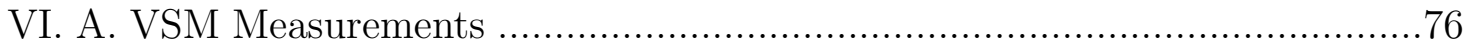

Chapter VII : Straintronics Device.............................................81

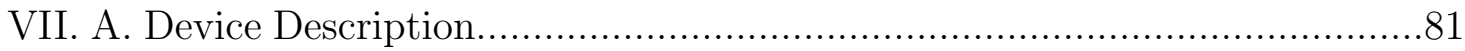

VII. B. SAW Resonator Characterization................................................ 84

VII. C. Nanostripes Characterization...................................................... 86

VII. D. Magnetic Structure of Ni Nanostripes............................................90

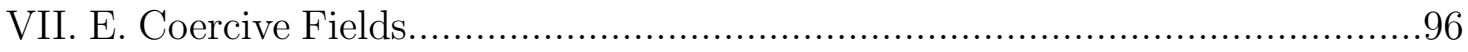




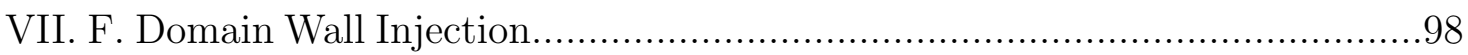

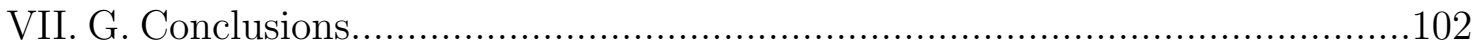

Chapter VIII : Effects of SAW's in Magnetostrictive Nanostripes ..............105

VIII. A. Characteristics of the Applied SAW's..........................................106

VIII. B. Effects of SAW's in Magnetoresistance of Ni nanostripes .....................108

VIII. C. DW Dynamics Assisted by SAW's...............................................116

VIII. D. MR Measurements on an AC Current ......................................... 121

VIII. E. Effects of SAW's in Magnetoresistance of FeCoB Nanostripes.................125

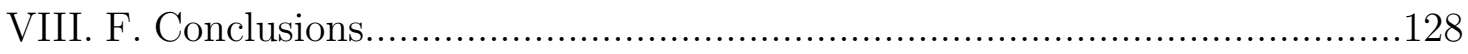

Chapter IX : SAW's-Induced Voltage in Magnetostrictive Nanostripes ......131

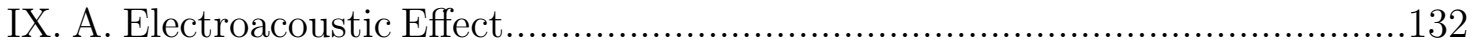

IX. B. Direct Measruement of the Induced Voltage in the Nanostripes.............135

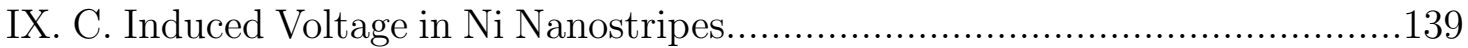

IX. D. Induced Voltage in FeCoB Nanostripes..................................................146

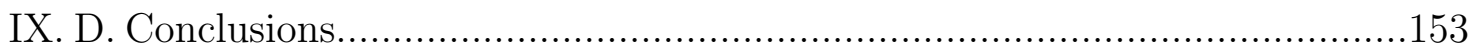

Appendix: Surface Acoustic Waves .................................................. 157

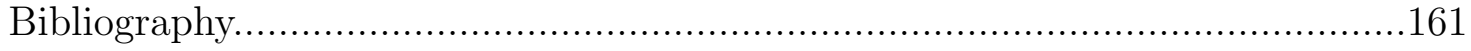




\section{INTRODUCTION}

The objective of this introductory chapter is to present a background of the spintronics field in order to offer the reader a context in which the development of our experiments constitute a logical step forward. This chapter consists in a brief summary of the current state of the spintronics field. A large amount of spintronics devices rely on the spin transfer torque (STT) for the control of magnetization states, however this effect typically needs high current densities in order to perform switching. This has pushed the scientific community in the pursuit for another paradigm to control and assist these processes. The concept of Straintronics is introduced as a new approach for the low power control of magnetization states of nanostructures via magnetoelastic effect (ME).

Spintronics is the area of condensed matter physics that studies the properties of the electron spin, with a view to improve the efficiency of electronic devices and to enrich them with new functionalities [Pullizi, 2012]. In this broad definition are included a wide variety of phenomena such as spin Hall effect (SHE), Rashba effect, Dresselhaus Effect, spin transfer torque (STT), giant magnetoresistance (GMR), tunnel magnetoresistance (TMR) among others. Spintronics emerged from discoveries in the 80's concerning spin dependent electron transport phenomena in solid state devices. This includes the observation of spin-polarized electron injection from a ferromagnetic metal to a normal metal by Johnson and Silsbee [Johnson, 1985] and the discovery of giant magnetoresistance independently by Albert Fert [Baibich, 1988] and Peter Grünberg [Binasch, 1988]. In the last decades the field has experienced a big boost, meanwhile the major problem which is the realization and fabrication of spintronics-based devices is gradually solved. Spintronics has already delivered functional devices (GMR read heads in large capacity hard disk drives), and magnetic RAM of insulator spintronics (magnetic tunnel junctions) is in an advance phase of development. An important line of research aims to the fabrication of memory devices based in magnetic structures delimited by different magnetic domains (bits) used to store information. The spin transfer torque is usually proposed in these devices as the mechanism that governs the processes of reading and writing via the injection of spin currents. 
Spin transfer occurs when a current of polarized electrons enters a ferromagnet, since there is generally a transfer of angular momentum between the propagating electrons and the magnetization of the film. This concept of spin transfer was proposed independently by [Slonczewski, 1996] and [Berger, 1996]. Experimental confirmation of STT was obtained when GMR was used to detect magnetization reversal in ferromagnetic multilayers with large current densities flowing perpendicular to the plane of the layers [Myers, 2000], [Grollier, 2001], [Wegrowe, 2001]. Subsequently, spin transfer has been implicated to explain the observation of spin precession for spin-polarized electrons that traverse a magnetic thin film [Weber, 2001] and enhanced Gilbert damping in magnetic multilayers compared to onecomponent magnetic films [Urban, 2001]. A great advance in STT research was reached in early 2004 by first demonstration of STT switching in $\mathrm{Al}_{2} \mathrm{O}_{3}$ based magnetic tunnel junctions (MTJ's) by [Huai, 2004]. The resistance of MTJ's depends on the relative orientation of magnetization in two ferromagnetic layers. This resistance change is called the tunnel magneto-resistance (TMR) ratio, defined by $\Delta R / \mathrm{R}=\left(R_{a p}-R_{p}\right) / R_{p}$, where Rap and $\mathrm{Rp}$ are the resistances for antiparallel (AP) and parallel (P) magnetization configurations between the two ferromagnetic films, respectively.

STT switching demonstrated in submicron sized MTJ's stimulated considerable interest for developments of STT switched magnetic random access memory (STT-MRAM) [Kishi, 2008], [Zhao, 2012]. STT driven by a radio-frequency (RF) electrical current were carried out in MTJ's in the work of [Sankey, 2008], where the magnitude of the STT is measured by ferromagnetic resonance (STT-FMR). The fast switching in current-induced magnetization precession also enables the fabrication of magnetic nanostructures to be new type of high frequency (microwave) tuneable nanoscale oscillators, namely spin-transfer nanooscillators (STNO's). They are frequency tuneable by current and magnetic field, low operation voltage and easy to integration on-chip [Rippard, 2005] [Mancoff, 2005], [Zeng, 2013].

A widely-spread experiment that takes advantage of the momentum transfer via STT and spin Hall effect (SHE) consists in the application of a RF current in a ferromagnetic/nonmagnetic bilayer [Liu, 2011]. In this experiment the RF current is forced to flow along the bilayer sample, while a dc voltage is generated across the sample due to the mixing of the RF current and the oscillating resistance. The non-magnetic layer must have strong spin-orbit 
scattering, since it has to convert the longitudinal charge current into a transversal spin current via SHE. When the RF current flows along the non magnetic layer, an oscillating spin current is injected into the ferromagnetic layer and when the resonance condition is reached, the spin current forces the precession of the spins within the ferromagnet, which generates a dc voltage across the sample due to the oscillating magnetization. Furthermore, the RF current generates an Oersted field that at the resonance condition also forces the precession of the spin in the ferromagnet. These two mechanisms that force the precession of the spins have two different contributions to the final de voltage signal. The separation of each contribution is used in many works to compute quantitatively the ratio of the spin current density entering the ferromagnet to the charge current density flowing through the non-magnetic layer. This ratio is usually known as the spin Hall angle. This procedure has been carried out in order to compute the spin Hall angle in a wide variety of hard metals, like Pt, Pd, Au and Mo [Mosendz, 2010]. Several parameters can be extracted out of this type of measurement, such as the spin mixing conductance in the bilayers [Yoshino, 2011] and the spin diffusion length in the hard metal [Roy, 2017]. Another mechanism that appears in this type of bilayer structures is the inverse spin Hall effect, which consists in the conversion of a spin current density into a charge current density due to the spin-orbit scattering. Experiments that bring out this type of mechanism use microwave excitations in microwave cavities in order to force the precession of the spins in the ferromagnet. The precession of the magnetization generates the emission of a transversal spin current from the ferromagnet to the hard metal. The spin current injected in the hard metal generates a longitudinal dc voltage due to the inverse spin Hall effect. This process is the counterpart of the SHE, and requires the same structures and the computation of the same parameters [Czeschka, 2011].

STT is also involved in the reading and writing processes that take part in proposed non-volatile memories like the Racetrack Memory (RM) [Parkin, 2004]. A RM is a memory device whose basic elements are magnetic nanowires (NW's). In the RM the nanowires are U-shaped and arranged perpendicularly to the surface of a silicon wafer. The magnetic domains of the NW's are used to store information with the data encoded as a pattern of magnetic domains along a portion of the NW's. Domain walls (DWs) are formed at the boundaries between magnetic domains magnetized in opposite directions along a racetrack. 
The spacing between consecutive DW's (bit length) is controlled by pinning sites fabricated along the NW's. The DWs in the magnetic racetrack can be read with magnetic tunnel junction magnetoresistive devices placed close to or in contact with the nanowires. Writing DWs can be carried out using the self-field of currents passed along neighboring metallic nanowires; using the spin-momentum transfer torque effect derived from current injected into the racetrack from magnetic nanoelements [Parkin, 2004].

Since the RM was first proposed, the amount of researches that have focused on the control of the motion, nucleation, pinning and depinning of DWs in nanostripes and nanowires has grown very rapidly. Nowadays the literature in this topic is extremely rich. One of the most promising achievements in the field was the controlled DW injection and pinning by single current pulses [Parkin, 2008], [Muñoz, 2011]. To this day all the works that study nanostripes and nanowires use STT as the only effect to control the magnetization dynamics. However STT still has a main disadvantage that remains to be solved, which is the high current densities required in these nanostructures for the DW motion assistance. Several works have shown that the necessary current densities are too high $\left(\sim 10^{7} \mathrm{~A} / \mathrm{cm}^{2}\right)$, e.g. in Permalloy (Py) [Parkin, 2007], [Thiaville, 2009], [Grollier, 2011] and [Lepadatu, 2012]. Nevertheless, this is not exclusive of nanostripes, for example, typical critical current densities for excitations in point-contact MTJ-devices are $10^{8}-10^{9} \mathrm{~A} / \mathrm{cm}^{2}$, whereas nanopillar MTJ-devices have achieved current densities of $<10^{7} \mathrm{~A} / \mathrm{cm}^{2}$. This has encouraged us to follow a line of work to explore the possibilities of magnetization dynamics assistance in magnetic nanostripes beyond the STT.

One of this novel direction of spintronics is called spin-orbitronics and exploits the spinorbit coupling (SOC) in non-magnetic materials instead of the exchange interaction in magnetic materials to generate or detect spin polarized currents. This opens the way to spin devices made of only nonmagnetic materials and operated without magnetic fields. The interplay between current-driven spin-orbit torques and chiral magnetic texture at transition metal interfaces has resulted in very fast current-driven magnetization reversal [Miron, 2011], [Liu, 2012], [Garello, 2014] and ultrafast domain wall propagation [Miron, 2011], [Yang, 2015]. Spin-orbit coupling can also be used to create new types of topological magnetic objects as the magnetic skyrmions [Romming, 2013], [Hsu, 2016]. 
Another new field that has developed rapidly in the last decade in this direction, is the possibility of control the magnetic properties via strain and deformation. This new field has been named as "Straintronics" and takes advantage of the magneto-elastic effect also known as the Villari effect, which is the change of the magnetic susceptibility of a material when subjected to a mechanical stress. The effects of static stress in magnetic materials have been known for many years, but only to put into perspective we comment some works that use stress to modify the magnetic behaviour of nanostructures; The works of [Zhukov, 2012] and [bhatti, 2018] showed an enhacement in the DW velocity due to static strain in FeCoB nano- and microwires, static strain can also achieved the decrease in coercive field in galfenol thin films [Li, 2014] and the work of [Finizio, 2014] proved the change in relative area domains of Ni nanosquares under a stress induced via magnetoelastic coupling. Since a large magnetostriction coefficient is a requisite for a strong magneto-elastic effect, we choose nickel $(\mathrm{Ni})$ and iron-cobalt-boron $(\mathrm{FeCoB})$, materials with a high magnetostriction of up to 100 ppm [Lee, 1971], [Diaz, 2012] in our experiments in nanostripes.

Nickel is not used very often when fabricating nanostripes or nanowires for studying magnetization dynamics, due to its crystalline anisotropy, magnetostrictive behaviour and polycrystalline structure which make it unsuitable to study DW dynamics, since the grain boundaries force unpredictable pinning along the nanostripes during the reversal process [Kozlov, 2019]. This makes Ni a difficult material for the DW injection and magnetization dynamics study at nanometer range. However, the choice for the $\mathrm{Ni}$ is based in its magnetostrictive behaviour which has been well studied and because of its well-known magnetic paramateres, which make it appropriate for simulation studies. Actually, in this manuscript we present the results of a series of magnetic simulations performed with the object oriented micromagnetic framework (OOMF) software, where Ni cylindrical-shaped nanowires are simulated with a chemical constraint (paramagnetic and non-magnetic) in the middle of it. Different types of DW (vortex and transverse) are initially nucleated in one of the ends of the nanowires. Pinning and depinning fields as well as the evolution of the DW's behaviour in the constraint and in motion are studied. Results of this simulations works were published [Castilla, 2017]. Other material more common in the fabrication of nanowires and nanostripes is permalloy (Py) [Beach, 2005], [Parkin, 2007], [Thiaville, 2009], [Munoz, 
2011], whose crystalline anisotropy is much lower and the DW dynamics is governed by the shape anisotropy, what makes it more suitable for the magnetization dynamics study.

The field of straintronics has recently started to explore the advantages of coupling surface acoustic waves (SAW's) with nanomagnets. SAW's are often generated at the surface of a piezoelectric substrate with the help of an interdigital transducer (IDT). The works of [Davis, 2010] and [Förster, 2016] show that SAW's induce an additional anisotropy in the nanomagnets in the direction of propagation of the wave. [Li, 2014] and [Thevenard, 2016] achieved the reduction of coercive fields in galfenol thin films and in (Ga,Mn)(As,P) thin films respectively in presence of SAW's. SAW's have also been shown to promote the switching in ferromagnetic films or nano-elements [Sampath, 2016] and [Tejada, 2017] to assist magnetic recording [Li, 20014] or to reduce the energy dissipation in spin transfer-torque random access memories [Biswas, 2013]. Additionally, as many of the modern spintronic devices are based on magnetic domain walls (DW), there have been interesting proposals to use SAW's to control the movement of DWs and indeed there has been a recent experimental demonstration of SAW assisted DW motion in films with perpendicular magnetic anisotropy [Edrington, 2018]. Nevertheless, despite being the ferromagnetic nanostripes the fundamental unit of many spintronic nanodevices, to our knowledge, there are still no experimental studies dealing with the interactions between SAW's and DW's in nanostripes. In this work we explore the effect of SAW's on the magnetization process of $\mathrm{Ni}$ and $\mathrm{FeCoB}$ ferromagnetic nanostripe.

From fundamental point of view, SAW's have been used to promote elastically driven ferromagnetic resonance in a thin film, in the work of [Weiler, 2011], where the SAW's are sent towards a ferromagnetic thin film and the power absorbed by the magnetic structure is measured as a function of the amplitude and orientation of an external magnetic field. In this work it is exposed that the absorption of the SAW's by the ferromagnet is dependent on the magnetization orientation, which in practice is a compromise between the anisotropy of the magnetic film and the Zeeman energy. Furthermore, it is conclude that the acousticallydriven resonance is excited by an internal RF magnetic field generated by the periodical strain in contrast to the classical FMR, which exploits an external RF magnetic field to drive the resonance [Thevenard, 2014]. SAW's have also been proven to pump spin current 


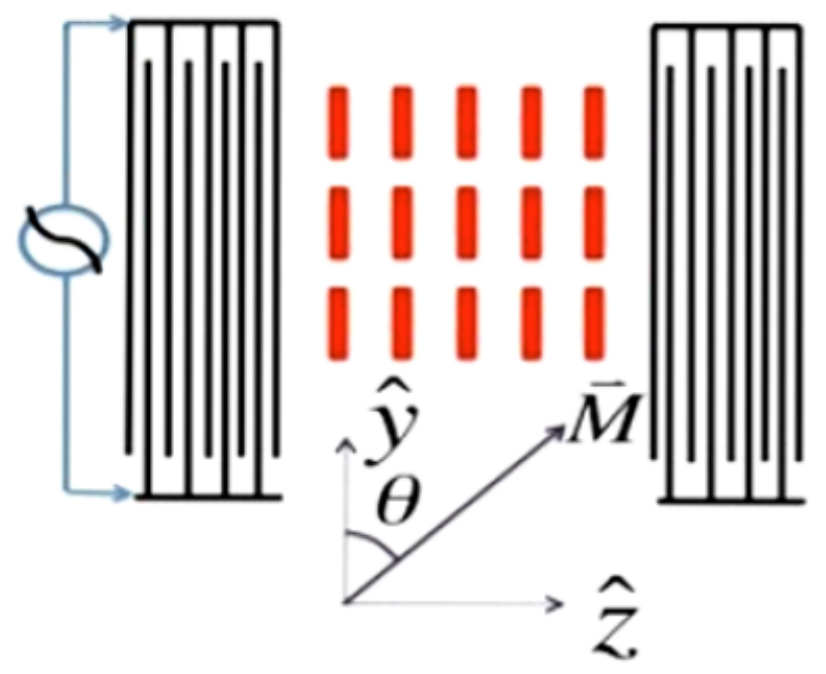

FIG. 1: Example of an experiment setup of the SAW's application in nanostructures. Rectangle nanomagnets are placed in front of a SAW device. [Davis, 2010].

in ferromagnetic/non-magnetic bilayers [Weiler, 2012]. This type of experiment takes advantage of the SAW's, similarly to an electromagnetic excitation in order to promote the precession of the spins within the ferromagnet. The precession of the spin can relax via emission of a spin current into the hard metal. This spin current can be measured by the inverse spin Hall effect, which generates a longitudinal dc voltage across the bilayer structrure. SAW's have also been used to detect delayed magneto-dynamic modes [Förster, 2016] or magnon-phonon dynamics [Berk, 2019], or even to measure the intrinsic Gilbert damping of a single nanomagnet .

This doctoral thesis is organized in nine chapters. Chapter two covers the experimental techniques employed to fabricate and characterize samples. Chapter three collects the results of the measurements performed in $\mathrm{Fe} / \mathrm{Gd}$ multilayers, consisting in ferromagnetic resonance and VSM measurements. Chapter four presents the results of the simulations performed by the OOMF software in cylindrical-shaped nanowires with a chemical constraint that studied the DW behaviour in them. Chapter five focuses on structural characterization of the piezoelectric layers $(\mathrm{ScAlN})$ and X-ray diffraction characterization of thin films whose structure and crystalline properties are analogous to the nanostripes'. Chapter six presents 
VSM measurements in thin films whose cyrstalline properties were studied in chapter five in order to obtain the anisotropies directions and constants. Chapter seven covers the description of the straintronics device by the characterization of the SAW resonator and the magnetoresistance of the different nanostripes as well as their DW injection efficiency. Chapter eight presents the effects of the application of the SAW's in the magnetic nanostripes through magnetoresistance measurements. In chapter nine is presented the electroacoustic effect, its consequences and the acoustically driven precession forced by the SAW's. 


\section{EXPERIMENTAL TECHNIQUES}

The goal of the chapter is to summarize the experimental techniques employed during the development of this research work. The experimetnal work was performed in the Institute for Optoelectronic Systems and Microtechnology of the Universidad Politecnica de Madrid (ISOM-UPM). Each section includes a brief description of the experimental techniques and the types of measurment performed. The nanodevices were created by a combination of Electron Beam Lithography and Sputtering deposition. Surface acoustic waves resonators were characterized by a Network analyzer. The structural properties of the samples were characterized by X-ray Diffractometry and the magnetic response was analyzed by Vibrating Sample Magnetometer. Magnetoresistance measurements were performed in a homemade setup in the magnetic devices group at UPM. FMR measurements were performed in the CNRS-THALES facilities in Palaiseau (France)

\section{A. Sample Nanofabrication}

All samples were produced at the ISOM-UPM facilities, which inclue a 100-100 class cleanroom where substrates preparation and nandovices were fabricated. The devices were fabrictaed over one type of silicon substrate, thermally oxidiezed $\mathrm{Si}(111)$, with a $\mathrm{SiO}_{2}$ thickness of $25 \mathrm{~nm}$. The silicon substrates were cut from commercial wafers using Kulicke and Soffa Precision Dicing System Model 780. Substrates 10 x 5 mm were used. In preparation to thin film fabrication, substrates were cautiosly cleaned with acetone ultrasonic cleaning and isopropyl alcohol (IPA).

\section{Magnetron Sputtering}

Sputtering is a physical deposition technique known since its discovery in 1852 . With the introduction of unbalanced magnetrons in the late 1980s, sputtering undergoes a rapid commercial diffusion, thanks to great performance improvements. The main advantage of magnetron sputtering is the high growth-rate achievable, which makes it one of the most convenient choice amongst thin film coating techniques. A target plate is kept at a negative potential of hundreds of volts in presence of glow discharged plasma. The plasma is produced by the ionization of an inert gas, which is generally Ar. However, it is also possible to use a 


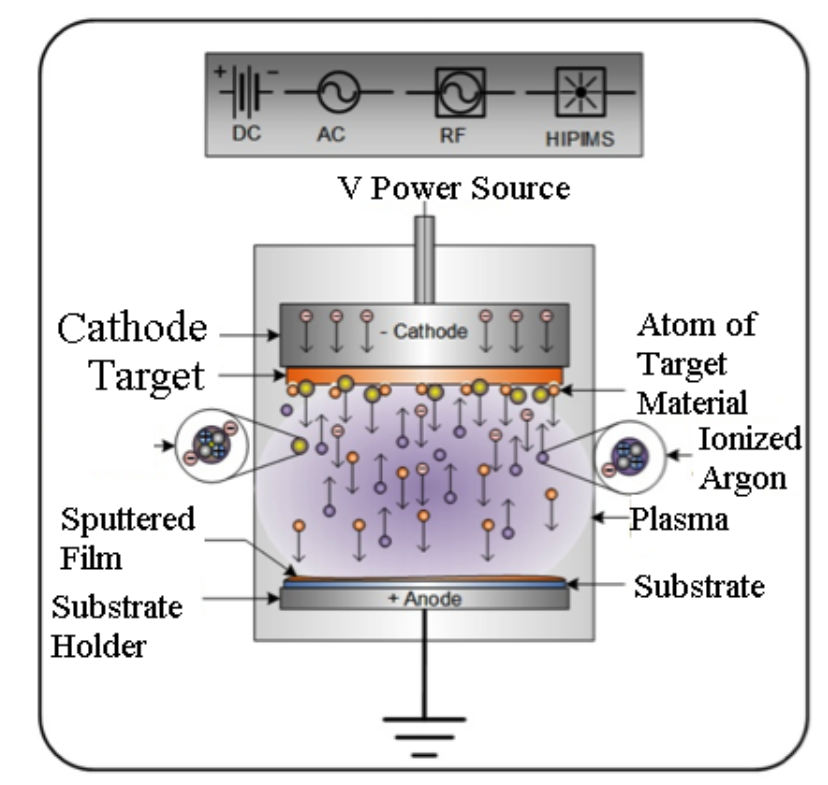

FIG. 2: Schematics representation of a magnetron Sputtering system [www.semicore.com]

non inert gas such as oxygen or nitrogen either in place of, or (more commonly) in addition to the inert gas (argon). When this is done, the ionized non inert gas can react chemically with the target material vapor cloud and produce a molecular compound which then becomes the deposited film. This is called, reactive sputtering. In order to deposit a non-conductive target, a radiofrequency $(\mathrm{RF})$ magnetron is required, since the applying of a DC negative potential will quickly induced a positive charge on the non-conductive target surface, a RF power allows to balance the surface charges when the positive potential is applied. The ionization of the plasma is increased by the presence of a magnetic field parallel to the target, produced by permanent magnets properly placed underneath the field lines, increasing the probability of electron-atom collisions by an order of magnitude. The energetic ions of the plasma are accelerated towards the target and the collisions result in the removal of target atoms, which eventually deposit on the substrate.

All the samples in this work were grown and deposited in two different sputtering systems in the ISOM-UPM facility. The first system consists of a main chamber which reaches a base pressure of $10^{-6}$ mbar and a comercial RF magnetron where a ScAl target is placed. The magnetron is positioned at the top of the vacuum chamber. The first step is the deposition of the ScAlN on our silicon oxide substrates. We deposit this piezoelectric material through the RF reactive sputtering. Nitrogen gas is pumped inside the chamber and its flux is 
regulated with a mass flow controller in order to control the pressure of Nitrogen insde. The target plate is ketp to a potential of hundreds of volts and the Nitrogen gets ionized in a plasma. The ionized Nitrogen can reacts chemically with the target material vapor cloud of ScAl producing a molecular compound which then becomes the deposited film of ScAlN.

The second system also consists in a main vacuum chamber which can reach a base pressure of $10^{-8}$ mbar with five DC magnetrons and a RF magnetron properly separated to avoid contamination among the targets. The magnetrons are located on top of the chamber, beneath them, the sample holder can rotate freely in order to be placed under the desired target of the material to be deposited. Two permanent magnets are present at opposite edges of the sample holder so to generate a magnetic field parallel to substrate surface. This magnet field is about 300 Oe and it is suffiecient to induce magnetocrystalline anisotropy in the sputtered magnetic materials.

Both systems have load lock chambers which allows to reach the base pressure in few minutes when loading a substrate. There is a large dependence between the growth rates of the materials and their distance to the sample holder. This may explain the reason why these growth rates are relatevely high compared to other works. Table I summarizes most of the materials deposited during this thesis, their growth conditions and their growth rates.

Stepping Motor in the Sputtering Chamber In this thesis we carried out the fabrication of multilayered samples. The thicknesses of individual layers fabricated are thin enough for us to require a high precission method able to rotate fast the position of the sample holder inside the sputteing chamber. In order to achieve this control, the sample holder is attached through a stem inside the chamber to a stepping motor, which is placed and handled from the outside of the chamber. The stepping motor is connected to a Stögra position control, model WSERS xx.230 AC V01, programmable via standard Windows-PC. This allows a precission in time of $0.1 \mathrm{~s}$ and $1^{\circ}$ in the position of the stepping motor. 


\begin{tabular}{||l|l|l|l|l||}
\hline \multicolumn{5}{|c|}{ Sputtering Targets and Growth Conditions } \\
\hline Target & $\begin{array}{l}\text { Magnetron } \\
\text { Type }\end{array}$ & Power $(\mathrm{W})$ & $\begin{array}{l}\text { Ar } / \mathrm{N} \quad \text { Pressure } \\
\left(10^{-3} \text { mbar }\right)\end{array}$ & $\begin{array}{l}\text { Grwoth rate } \\
(\mathrm{nm} / \mathrm{s})\end{array}$ \\
\hline \hline $\mathrm{Cr}$ & DC & 80 & 7 & 0.31 \\
$\mathrm{Ga}$ & DC & 60 & 7 & 1.21 \\
$\mathrm{Au}$ & DC & 60 & 7 & 1.06 \\
Fe & DC & 80 & 7 & 0.24 \\
FeCoB & Pulsed & 100 & 7 & 0.08 \\
Ni & DC & 80 & 7 & 0.18 \\
Pt & DC & 60 & 7 & 0.67 \\
Ta & DC & 60 & 7 & 0.52 \\
ScAlN & RF & 500 & 4 & 1.27 \\
\hline
\end{tabular}

TABLE I: List of materials deposited by sputtering in the thesis and their growth conditions and rates.

\section{Electron Beam Lithography}

Electron-beam lithography (often abbreviated as e-beam lithography) is the practice of scanning a focused beam of electrons to draw custom shapes on a surface covered with an electron-sensitive film called a resist. The purpose, as with photolithography, is to create very small structures in the resist that can subsequently be transferred to the substrate material. The underlying physical mechanism relies on the fact that the recording medium, typically a thin organic polymer film (resist), is altered by the passage of fast electrons. The electron beam changes the solubility of the resist, enabling selective removal of either the exposed (positive resist) or non-exposed (negative resist) regions of the resist by immersing it in a solvent. The process of the removal of the selected resist is called developing. The result is a patterned film, which acts as a binary mask for further processing.

After the developing process with a positive resist, the usual procedure is the growth/deposition of the materials which the nanodevices are made of. Throughout this 
thesis all growths after the e-beam procedure are carried out by sputtering deposition. By superimposing multiple pattern layers, an enormous variety of useful devices can be fabricated. The primary advantage of electron-beam lithography is that it can draw custom patterns (direct-write) with sub-10 nm resolution. This form of maskless lithography has high resolution.

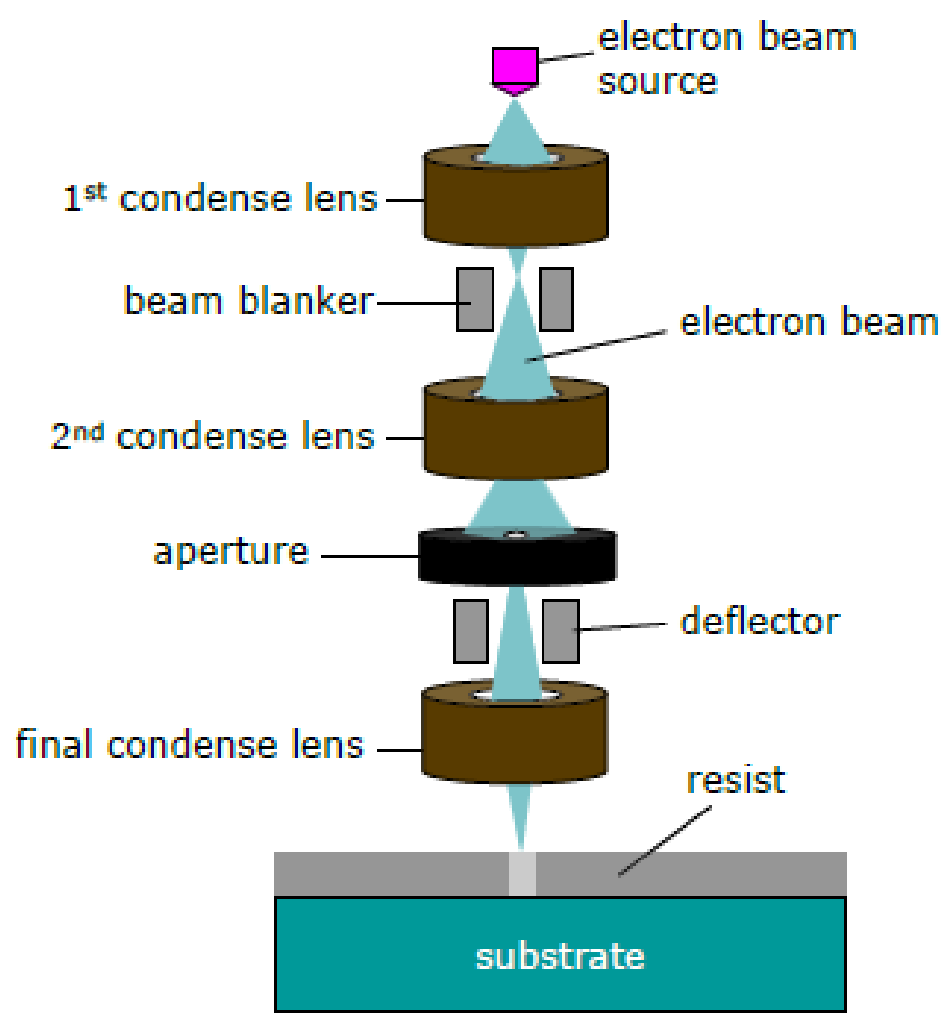

FIG. 3: Schematics diagram of an electron optical column to produce, blank and focus the electron beam. [Pimpin, 2012]

The electron beam lithography system used in this thesis is a CRESTEC cabl-9000C series $50 \mathrm{kV}$ system and is comprised of several subsystems, including:

1. An electron optical column, to produce the focused e-beam.

- E-beam lithography systems use electric and magnetic electron lenses to form a sharply focused e-beam, which is scanned over the writing surface. 
2. Analog electronics to produce, focus, blank/unblank, and scan the beam.

- The e-beam scans over the writing surface using electric fields, turning on and of while scanning. Fig. 3 presents the optical column with its magnetic lenses and blankers needed to focus and control the diameter of the electron beam.

3. Digital electronics to store and transmit the pattern data.

- The pattern data are typically created using CABL-2000 software for computer design. These data must then be turn into a format usable by the e-beam writer. A digital electronic data path automatically converts and sends the data to the e-beam writer.

4. A high-precision mechanical XY stage to position the writing substrate relative to the e-beam.

- The XY stage moves under the optical column in order to draw the pattern in the resist. The stitching accuracy depends on the precision of the stage in XY.

5. A high-vacuum system, with provision to move the writing substrate in and out of the vacuum.

- A prevaccuum chamber is part of the system in order to introduce the samples and not breaking the high vacuum in the internal chamber.

6. High-speed computers and microprocessors, to automatically perform all of the necessary tasks.

7. An extensive software system

A considerable engineering effort is needed to make all of these components work reliably together. 


\section{Nanofabrication Procedure}

As we stated before, the advantage of fabricating by EBL system is the possibility of an infinite variety of devices made by different metals by superimposing different nanolithography phases. In this section we will explain which is the procedure to follow during this thesis to the growth phase of the device. The procedure of fabricating devices from start is as follows:

First, we spin coated a resist, PMMA/ZEP, which are positive resists, over a substrate of $\mathrm{SiO} 2 / \mathrm{ScAlN}$ at $5000 \mathrm{rpm}$. Then, the specimen is baked in order to evaporate the solvant of the resist. As the substrate has insulating behaviour and in order to avoid that it gets charged during the exposure in the electron beam lithography, a conductive polymer is spin coated over the resist. The sample undergoes an electron exposure with a defined pattern in the EBL system, which alters the solubility of the polymer. Once the pattern is drawn in the resist sample, this is immersed in a liquid called developer, whose task is remove the exposed (in case of positive resist) polymer. When the exposed pattern is removed a binary mask is obtained. Then the growth procedure is performed and the metals are deposited by sputtering deposition. Once the deposition of the metal is performed. The excess of resist and metal is eliminated by immersing the sample in an organic disolvant, which is acetone in case of PMMA resist and 3-metylpirrolidone in case of ZEP resist.

a)

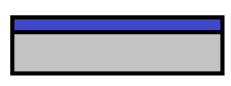

d)

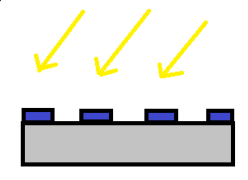

b)

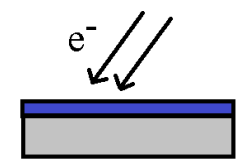

e)

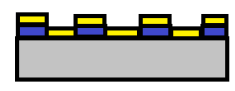

c)

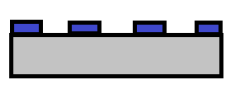

f)

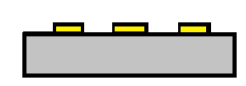

FIG. 4: Representation of the nanofabrication steps: a) The resist is spin coated over the substrate, b) The resist is exposed by electron beam c) The exposed resist is removed, developing step, d), e) The metal is deposited by sputtering and f) The resist and excess of metal are removed by immersing the sample in an organic disolvant (lift-off) 


\section{B. Structural Characterization}

The magnetic properties of the nanodevices studied trhoughout this thesis are dependent on their structural characteristics. The study of samples through X-ray diffraction relates the crystallographic properties of the samples with their magnetic properties.

\section{X-ray Diffraction}

X-Ray crystallography is an experimental technique used for studying structural order, based on diffraction of X-rays by long range ordered atoms. The interaction between X-rays and atoms are mostly elastic scattering due their electronic shells. Scattered X-rays add constructively when the material has crystallographic ordered and they cancel out if the atoms in the material are randomly positioned by desctructive interference. The specific directions, where they add constructively are determined by Bragg's law:

$$
n \lambda=2 d \sin \theta
$$

where $d$ is the lattice spacing of the crystal, $\theta$ is the incidenet angle, $n$ is any integer, and $\lambda$ is the wavelength of the incident $\mathrm{X}$-rays.

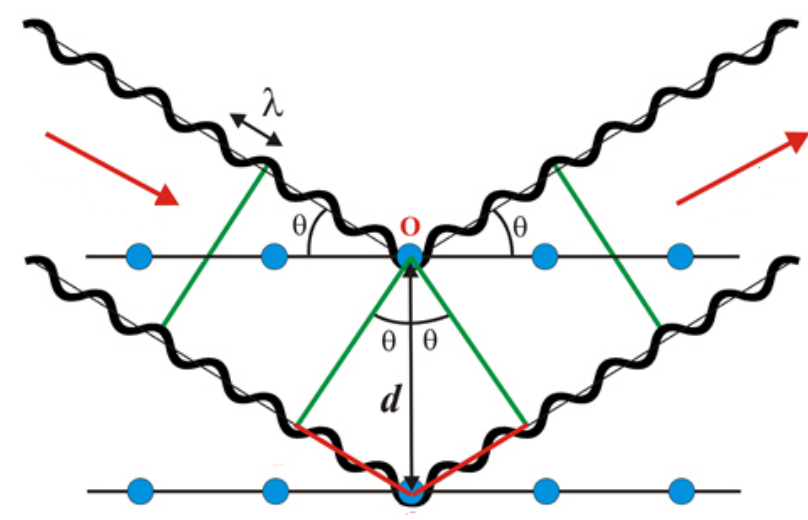

FIG. 5: Schematic diagram of Bragg's diffraction

Structural characterization with an X-ray diffractometer consists in determining the lattice spacing $d$ of a crystalline sample by using X-rays of known wavelength and measuring for which $\theta$ is fulfilled. 


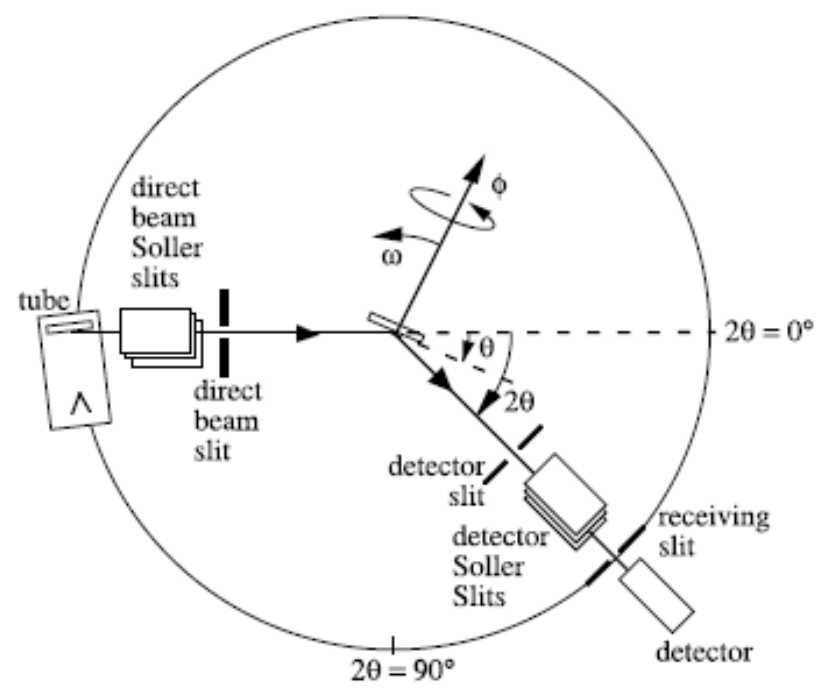

FIG. 6: Schematics diagram of a X-ray diffractometer [Fultz, 2013]

A schematic representation of an X-ray diffractometer (XRD) is shown in Figure 6. The $\mathrm{XRD}$ is mounted on a goniometer which allows both source and detector to rotate around the sample holder. Coherently with the Braggs notation, the incident angle is $\theta$ and the diffraction angle with respect to the incident direction is $2 \theta$. Note that when source and detector rotate at the same speed but in opposite direction, the Bragg plane is fixed with respect to the sample holder. On contrary, when they move at the same speed and in the same direction, the Bragg plane rotates with respect the sample holder, while the Bragg angle is kept constant. The sample holder is mounted on a goniometer with six degrees of freedom. The relative angle $\omega$ describes the rotation of the sample holder around the diffractometer axis. The rotation around the out-of-plane axis is described by the angle $\phi$, while $\psi$ is the tilting angle. The beam source is an X-ray tube, where highly energetic electrons are accelerated toward a metal target, which then emits X-ray according to the characteristic spectrum of the metal. The incident beam optics consists of a series of slits which control the divergence angle and the spot size of the X-ray beam. A monochromator is often used to select one emission peak of the characteristic spectrum. The beam is directed to the sample at the center of the diffractometer and the diffracted signal is collected by the detector. 
There are several types of techniques of X-Ray measurements, that depend on the geometry and angles between the incident and reflected or diffracted beams and the sample, which allows the structural characterization of thin films. Here,only the relevant ones are presented.

Out-of-plane $\theta / 2 \theta$ scan This is a standard characterization for thin films. It consists in rotating both source and detector at same speed and opposite directions. By this method, Bragg planes are kept constant. If the system is aligned with the sample, Bragg planes will be parallel to the sample surface. This technique will analyze the crystal texture in the out-of-plane direction. By this configuration the volume of the sample that is irradiated, diminishes as the incident beam angle increases.

Grazing Incident Angle (GID) In this measurement the incident angle is kept constant, around 1 degree and we perform a $2 \theta$ scan with the detector. The idea is to obtain the diffraction of the planes that are not parallel to the sample surface. Doing this we can rotate the Braggs planes as the detector moves. The sample surface is kept still and is never parallel to the Bragg planes. This technique is suitable for non-textured polycrystalline materiales. By this technique the irradiated volume is larger, because it only depends on the incident angle, which is very low. Out-of-plane $\theta / 2 \theta$ and GID measurement give complementary information about the structure of materials.

Rocking curves It consists in keeping both the beam source and the detecter at fixed positions while "rocking" the sample holder by varying the angle $\omega$. The system is firstly located at Bragg condition in the sample while the sample is being rotated around the bragg condition. This measurements gives information about how the planes are spread around the direction parallel to the sample substrate. This is known as the mosaicity.

X-Ray reflection In order to measure the thickness or thin films, the sample is placed with a very low incident beam angle below the critical angle, where no beam is diffracted by the sample. The detector is also located in a ver small angle so that the Bragg's condition is maintained. The information obtained can be usefuel in the measurment of bilayer thickness repetition 
The system used for XRD measurements during this research work are a PANalytical X'pert Pro diffractometer of ISOM-UPM. This system use a $\mathrm{Cu}$ target which emits $K \alpha 1$ $\mathrm{X}$-rays whose wavelength is $1.541 \mathrm{~A}$

\section{Network Analyzer}

A network analyzer is an instrument capable to measure properties of electrical networks, specially those associated with reflectivity and transmission of electrical signals known as scattering parameters or S-parameters. They are often used to characterize two-port networks such as amplifiers and filters, but they can be used on networks with an arbitrary number of ports. Network analyzers can measure and display a device under tests (DUT) magnitude and phase information across a frequency range. In essence, a network analyzer characterizes a DUT in terms of scattering parameters, or S-parameters.

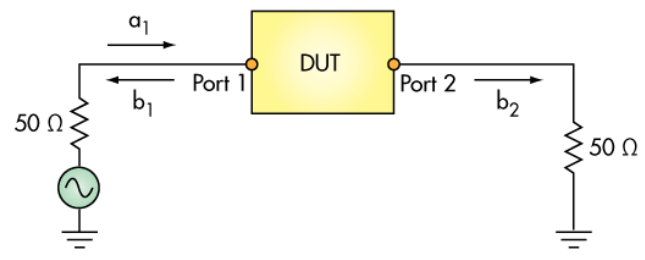

FIG. 7: Schematics diagram of network analyzer

The network analyzer used to characterize the resonator in this work is Agilent PNA-L model N5230A with four ports and a range spectra of $300 \mathrm{kHz}-20 \mathrm{GHz}$. The Network analyzer is embeded in a system that consists in an analytical probe-station Cascade Microtech 9000. Coaxial cables W.L. Gore 3GW40 connect the ports with the probe heads. The probe heads are locked/placed in a micropositioner with one inch movement in all axes and captured cross-roller bearings to prevent accidentally moving the tip.

S-Parameters S-parameters are used to characterize performance at RF and microwave frequencies in terms of incident and reflected waves. S-parameters are vector quantities, meaning they contain both magnitude and phase information. A scalar network analyzer (SNA) can only measure magnitude, while a vector network analyzer (VNA) can measure both magnitude and phase. 


$$
\left(\begin{array}{l}
b_{1} \\
b_{2}
\end{array}\right)=\left(\begin{array}{ll}
S_{11} & S_{12} \\
S_{21} & S_{22}
\end{array}\right)\left(\begin{array}{l}
a_{1} \\
a_{2}
\end{array}\right)
$$

where $a_{i}$ is the singal applied to the DUT from the port $i$ and $b_{i}$ is the signal measured in the port $i$. S-parameters are complex matrix that show Reflection/Transmission characteristics (Amplitude/Phase) in frequency domain. Two port device has a four S-parameters. As stated earlier, S-parameters contain both magnitude and phase information. Magnitude is typically expressed in decibels $(\mathrm{dB})$. This is mathematically defined as:

$$
S_{i j}(d B)=20 \log \left|S_{i j}\right|
$$

For the goal of this thesis work, the Reflection coefficient $\left(S_{11}\right)$ is the only coefficient measured in the fabricated devices, which is the reflection of the device connected to port 1. As only one port is used the reflected signal is $b_{2}=0$, therefore $S_{11}=b_{1} / a_{1}$. The dB representations of $S_{11}$ is known as return loss, which is the ratio in dB between the reflected signal and the incident signal. The usual representation of this parameter is by a graph that features power versus frequency. Figure 8 is an example chart of a typical resonator device measured throughout this project.

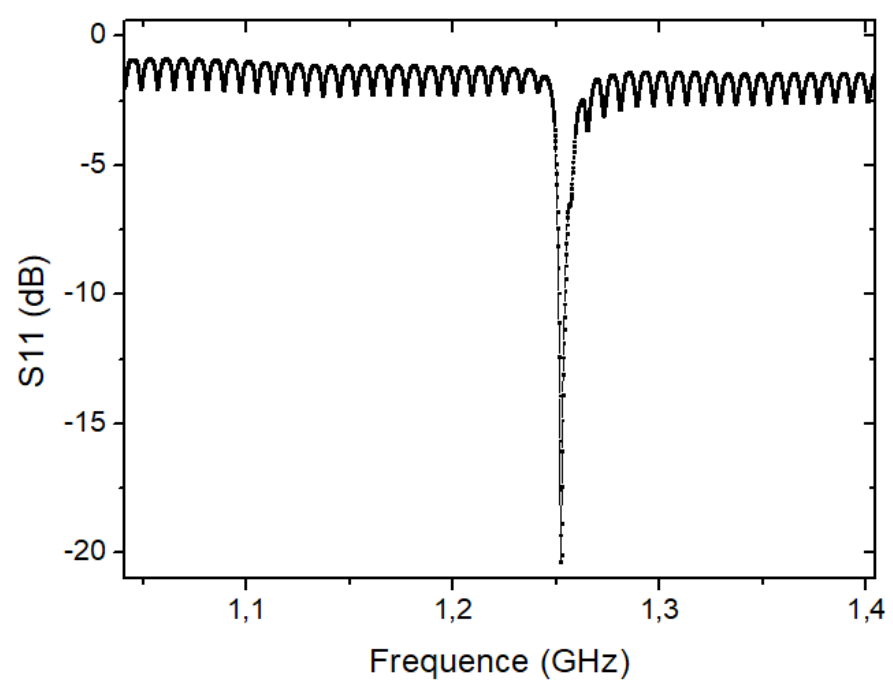

FIG. 8: Typical measurement of $S_{11}$ parameter of a SAW resonator.

We notice that there is a valley on $S_{11}$. That valley represents the frequency range at 
which the device reflects the least amount of power.

Smith Chart The network analyzer uses a Smith chart to show complex impedance over a specific range of frequencies. Smith chart can be used to show all kinds of complex quantities. If we look at a Smith chart for complex impedance (see figure 9), from left to right, we have 0 ohms going all the way up to infinity ohms. This line represents pure resistance impedance. Phase angles that lie above this line represent inductive quantities and phase angles that lie below this line represent capacitive quantities.

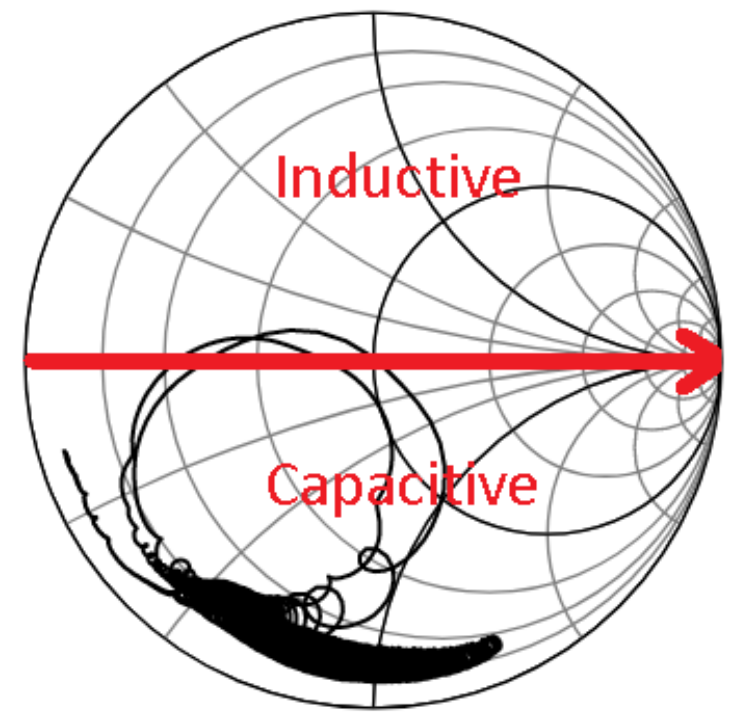

FIG. 9: Typical Smith Chart measured of one resonator during this thesis. The red line is the pure resistance impedance line, which divides the chart in two hemispheres of capacitive and inductive behaviour.

Close loops in a Smith Chart for a range of frequencies represent a valley or resonance in the reflection parameter $S_{11}$. The point of intersection of the loop corresponds to the two frequency having the same impedance. The point directly opposite to the knot of the loop represents the frequency of resonance of the DUT. As two frequencies have the same value of impedance and then the value of impedance changes (increases/decreases) and then return back to the same value results in the loop formation. The larger the difference between the frequencies whose impedance is same, greater will be the bandwidth. 


\section{Magnetic Characterization}

The line of work followed in this thesis was encouraged by a simulation study that described the DW dynamics in Ni nanowires. The software used for the study as well as a description of the simulations is commented in this section. For the straintronics part, we have analyzed the anisotropy of the grown-by-sputtering nanostripes by measuring the hysteresis loops of identically structured thin films, with a vibrating sample magnetometer (VSM). We also described the resonance modes in multilayered Fe/Gd samples by ferromagnetic resonance (FMR). Finally, by carrying out magnetoresistance measurements (AMR) in the magnetic nanostripes the magnetization states of them can be infered. The descriptions of the setup experiments of these techniques are presented in each subsection.

\section{Micromagnetic Simulations}

Micromagnetic simulations is an useful way to understand the processes that govern the magnetization dynamics observed in experiments. At micro- and nanoscale these are described through the Landau-Lifshitz-Gilbert equation (LLG), which is an ordinary differential equation in time. We perform the simulations using the object oriented micromagnetic framework (OOMF) software. This software allows to mesh the space in several cells of defined-by-user dimensions, define the magnetization $(\vec{M})$, exchange constant between cells $(A)$, magnetocrystalline anisotropy $\left(K_{c}\right)$ and let the system reach the stability through the LLG equation from an initial defined-by-user magnetization state. The simulations carried out in this work were performed in a $50 \mathrm{~nm}$ circular-section and $1 \mu \mathrm{m}$ long nanowires with parameters of saturation magnetization $\left(M_{s}=4.9 \cdot 10^{5} \mathrm{~A} / \mathrm{m}\right)$, exchange energy $\left(A=9 \cdot 10^{-12}\right.$ $J / m)$ and anisotropy constant $\left(K=5.7 \cdot 10^{3} \mathrm{~J} / \mathrm{m}^{3}\right)$ that emulates the Ni. Mid length in the nanowire there is a section with different composition (chemical constraint). For a Ni nanocylinder $50 \mathrm{~nm}$ in diameter, there is no a clear energetically favored type of DW, and both transverse and vortex DWs can be present. By fixing the spins at the edge of the NW at the start of the simulations it is possible to choose the type of DW initially nucleated. This simulation work studies the pinning and depinning fields at the chemical constraint as a function of the nature of this and the type of DW pinned in the constraint. Results of this study were published in [Castilla, 2017]. 


\section{VSM measurements}

Vibrating sample magnetometer (VSM) is an instrument able to measure the magnetization of a sample as a function of an external applied magnetic field. The principle behind its functioning is based in the Faraday's law of induction.

$$
\varepsilon=-N \frac{d \phi_{B}}{d t}
$$

Where $\varepsilon$ is the electromotive force induced in a coil of $N$ turns, when it experiences a variation of the magnetic flux $\phi_{B}$ with time t. The instrument is composed by these elements:

- Four detection coils

- A non-magnetic rod

- A water-cooled electromagnet

- A 360 degrees rotatable table

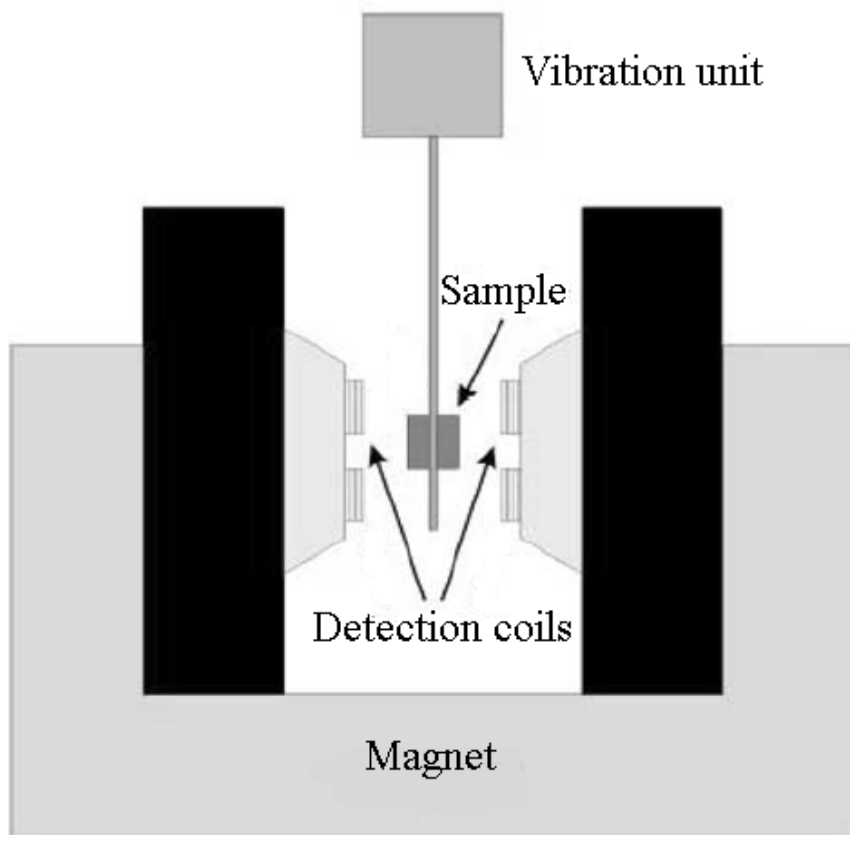

FIG. 10: Schematics diagram of a VSM [J. Toniolo, 2006] 
In presence of a magnetic sample, the magnetic flux that the coil experience is proportional to the magnetic moment of the sample. The principle of operation of a VSM is based on the observation of the voltage induced in a detection coil as the flux changes when the sample position oscillates. The induced voltage in the detection coil is proportional to the sample's magnetic moment, but does not depend on the strength of the applied magnetic field. A uniform DC magnetic field is generated by a water-cooled electromagnet. Four detection coils are placed in a fixed position to avoid unwanted vibrations. The sample is placed at the end of a rod and positioned in the center of the magnetic field. The rod is connected to a vibrator head which generates a sinusoidal oscillation perpendicular to the magnetic field lines. The oscillation of the magnetic sample generates a field distortion and hence a change in the magnetic flux experienced by the detecting coils. The voltage output from the coils is processed to extract a value of the magnetic sample. The rod can rotate freely, allowing to measure in-plane and out-of-plane directions. A LakeShore Model 7304 of the ISOM-UPM was used for hysteresis loop measurements at room temperature. This system hs a sensitivity of $5 \cdot 10^{-6} \mathrm{emu}$ and can reach $1.5 \mathrm{~T}$.

\section{Ferromagnetic Resonance}

Ferromagnetic resonance, or FMR, is a spectroscopic technique to probe the magnetization of ferromagnetic materials. It has been carried out using microwave cavities since the late 1950s, typically on bulk samples. Over the last decade, the advent of broadband FMR spectroscopy, using either stripline or coplanar waveguides (CPWG), has allowed for measurements continuously spanning several 10s of GHz. Such broadband measurements allow for significant improvements in accurately extracting the material parameters including the gyromagnetic ratio $(\gamma)$, damping $(\alpha)$, inhomogeneous broadening $\Delta H_{0}$, exchange stiffness (A). The classical description of microwave magnetization dynamics is provided by the Landau-Lifshitz-Gilbert (LLG) equation (10) shown in Figure 11,

$$
\frac{d \vec{m}}{d t}=-\gamma \vec{m} \times \mu_{0} \vec{H}_{e f f}+\alpha \vec{m} \times \frac{d \vec{m}}{d t}
$$

where $\vec{M}$ is the magnetization vector, $\gamma$ is the gyromagnetic ratio, $\mu_{0}$ is the permeability 


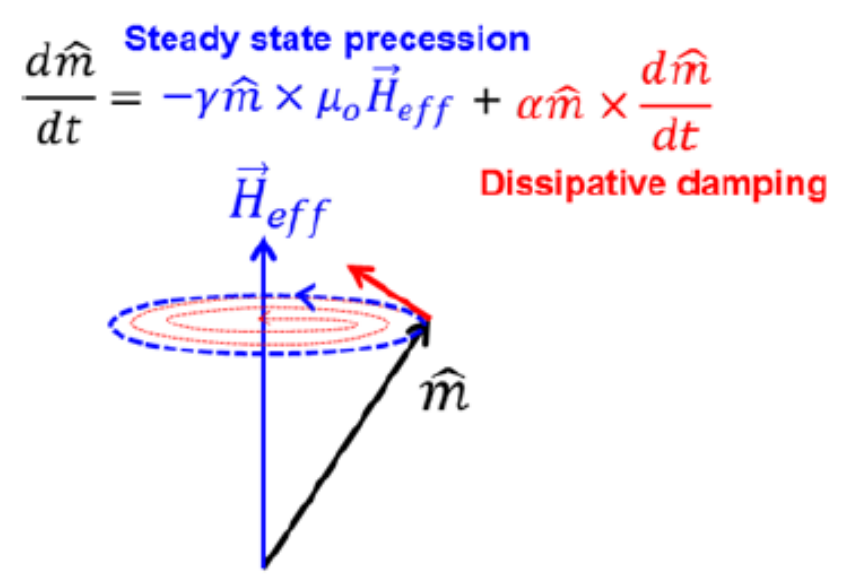

FIG. 11: Magnetization dynamics as described by the Landau-Lifshitz-Gilbert equation.

of free space, $H_{e f f}$ is the effective magnetic field, and $\alpha$ is the phenomenological Gilbert damping coefficient. FMR arises from the precessional motion of the magnetization $\vec{M}$ of a ferromagnetic material in an external magnetic field $\vec{H}$. The magnetic field exerts a torque on the sample magnetization which causes the magnetic moments in the sample to precess. The precession frequency of the magnetization depends on the orientation of the material, the strength of the magnetic field, as well as the macroscopic magnetization of the sample. The dissipative forces represented by Gilbert damping coefficient $\alpha$ causes the magnetization to spiral inwards and eventually point parallel to $H_{\text {eff }}$. By providing a microwave RF stimulus, the magnetization is pumped to keep precessing while absorbing the RF signal.

All FMR measurements were made in a Quantum Design Physical Property Measurement System (PPMS). The setup of the FMR spectroscopy measurements is formed by a coplanar wave guide (CPWG) whose role is to transmit a microwave signal from a radiofrequency (RF) source over a range of frequencies. Samples are glued side down to the CPWG in order to maximize the absorption of the microwave RF magnetic field created by the RF signal. In order to avoid CPWG and the sample shortout each other, samples are glued with a spin coating photoresist. When an external magnetic field $H_{D C}$ is swept through the resonance condition, the magnetization will begin to resonantly precess and will absorb energy from 
the CPWG.

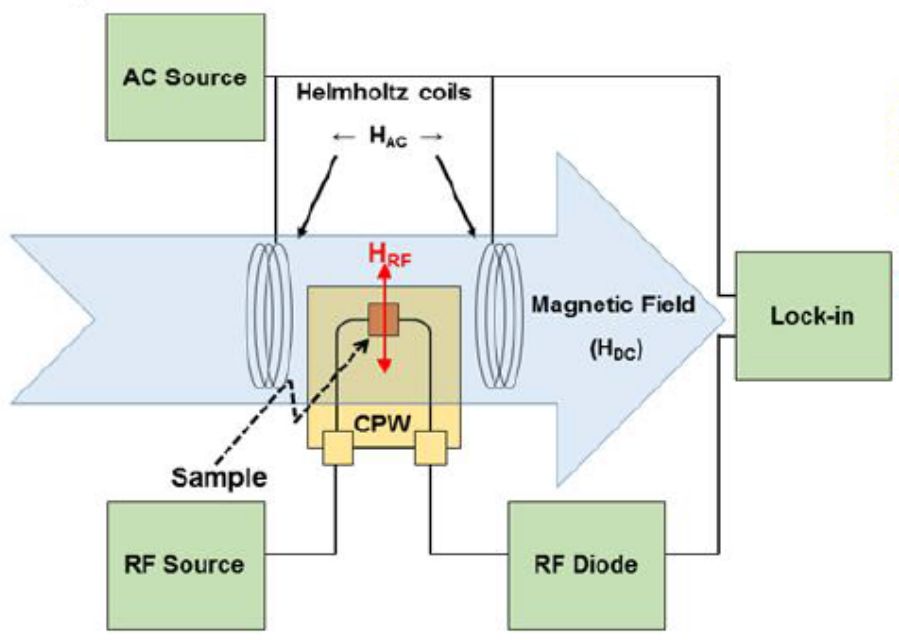

FIG. 12: Schematic of the FMR measurment set up with the critical components are highlighted, [qdusa.com]

This decrease in transmitted RF energy when sweeping through the resonance field is converted to a $\mathrm{DC}$ voltage by a broadband $\mathrm{RF}$ diode. In order to improve the signal-to-noise ratio (SNR), a lock-in detection technique is often employed, which requires modulating the signal at a known frequency. This modulation is provided by an additional set of Helmholtz coils powered by an AC source. This measurement scheme actually measures the derivative of the transmitted power.

\section{Magnetoresistance measurements}

The magnetoresistance is the tendency of a ferromagnetic material to change its resistance in presence of an external magnetic field. Anisotropic magnetoresistance (AMR) is the change of magnetization due to the angle between the magnetization of the sample and the electrical current that flows through it. This effect arises from the simultaneous action of magnetization and spin-orbit coupling and its detailed mechanism depends on the material. All the magnetoresistance measurements are performed in a circuit board (CB), where the sample is previously glued with a photosensitive resist at $150{ }^{\circ} \mathrm{C}$. The $\mathrm{CB}$ consists in 
sixteen gold contacts, electronic circuits, which are printed directly onto the CB, and SMA connectors. The printed circuits connect the gold contacts with the SMA connectors. The nanodevices in the sample and the gold contacts in the $\mathrm{CB}$ are connected via wire bonding with $17 \mu m$ diameter gold wires. The sample on the CB is placed between two small homemade electromagnets, which are able to generate magnetic fields up to 1000 Oe. The electromagnets are powered by a current source KEPCO bipolar operational power supply. A DC voltmeter (Keithley model 2002) and DC current source-meter (Keithley model 6430) are connected in parallel to the device to measure the resistance. Resistance of the device is measured by making pass through the device a known current and acquiring the voltage with the nanovoltimeter. In order to inject current pulses into the device, a constant voltage pulse generator (Agilent Pulse-Pattern Generator, model 81130A) is used. Bias tees (Picosecond Pulse Labs, model 5547A, 5540B) is used as required.

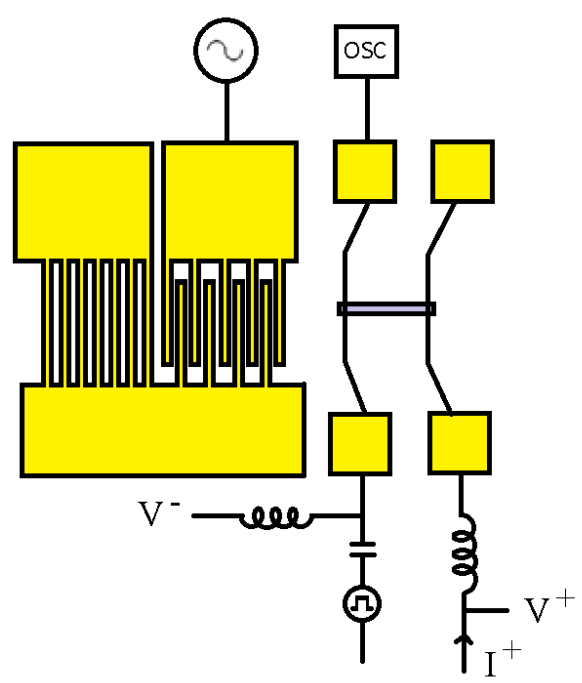

FIG. 13: Schematics of a set up for the magnetoresistance measurements, which includes the device formed by the nanostripe in front of the SAW resonator. Nodes in the connections of V, I and the pulse signal represent the Bias tees.

The SAW resonators are connected to a MXG Analog Signal Generator Keysight model N5181B, with a frequency range $9 \mathrm{KHz}-6 \mathrm{GHz}$. A digital storage osciloscope Agilent infiniium DSO90254A is used to display the injection pulses as well as the input surface acoustic wave signal. A schematics of the complete device and all of its connections for AMR measurements and pulse injection is depicted in Figure. 13 


\section{Measurements of the induced voltage.}

Complete study of the consequences of the application of SAW's to the magnetic nanostripes requires measurements in absence of any applied current to the nanostripes. For these measurements, our samples are glued to the circuit board in a similar setup as the magnetoresistance measurements. We observe that the sole application of the SAW's induces a voltage in the nanostripe, therefore it is necessary to isolate this effect from the voltage signal due to the nanostripe resistance. Lock-in amplifiers are used to detect and measure very small AC signals, all the way down to a few nanovolts. Accurate measurements may be made even when the small signal is obscured by noise sources many thousands of times larger.

Lock-in amplifier Stanford research systems, model SR830 DSP is used to enhance the sensitivity of the acquisition of data. Once the signal goes through the lock-in amplifier is picked by the nanovoltimeter for the posterior acquisition of the induced voltage. The RF source signal applied to the SAW resonator in this experiment is modulated at $1 \mathrm{kHz}$. Figure 14 shows a schematics of the set up used for this type of measurements.

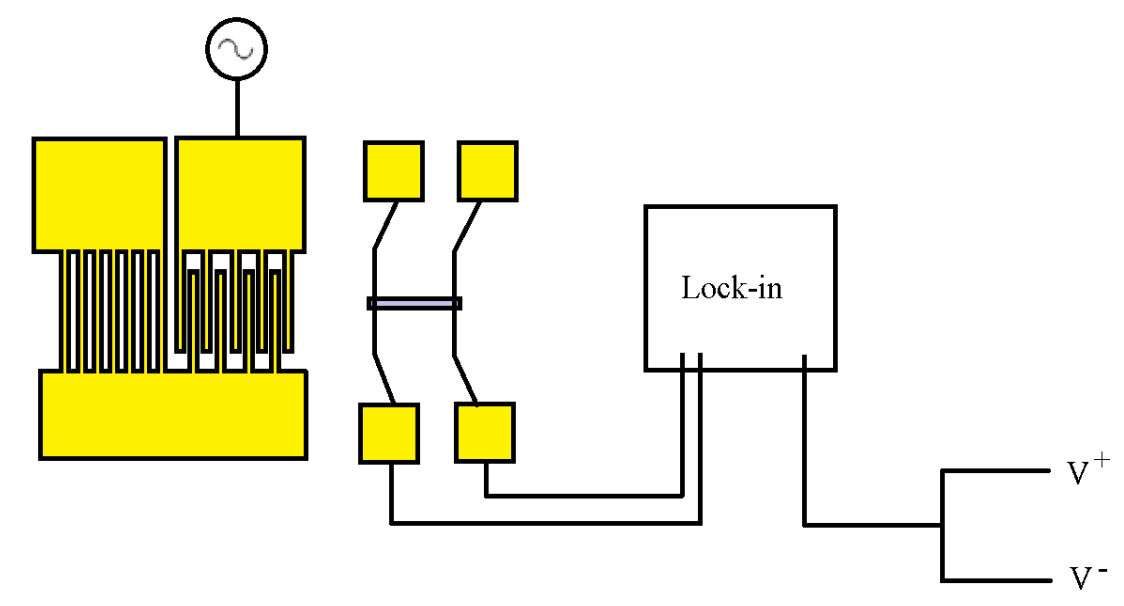

FIG. 14: Schematics of the set-up for the measurement of the effect of the application of SAW's on the nanostripes. A lock-in amplifier is used to improve the acquisition of the signal. 


\section{FERROMAGNETIC RESONANCE ON FE/GD MULTILAYERS}

Magnetic layered structures have been an object of extensive research in the last decades. The periodic stacking of two distinct ferromagnetic materials gives rise to a variety of magnetic exchange interactions. Magnetic multilayers composed of a rare-earth (RE) element, such as $\mathrm{Gd}$, and a transition metal (TM) like Fe, Co and $\mathrm{Ni}$ are interesting example systems. Due to their very different ordering temperatures, magnetic configurations depending on the structural parameters may occur. The Fe/Gd is one of the most interesting systems since it posseses a very rich magnetic state diagram. Depending on the temperature $T$, the external magnetic field applied $H$ or the ratio between the thickness of the different layers, these systems have different magnetic orders, based on which of both materials alings with the external magnetic field, since the coupling between the different layers is antiferromagnetic.

In weak magnetic fields antiparallel coupling between Fe and Gd prevails, with either the Fe layers magnetization oriented in the direction of the external field ( $\mathrm{F}$ state) or the Gd layers magnetization oriented along the external magnetic field ( $\mathrm{G}$ state). On the other hand, when the magnetic field applied becomes stronger there must be a compromise between the minimization of the Zeeman energy and the AFM coupling in the interlayer. In this situation only the magnetization of the bulk of the layers lies parallel to the external magnetic field, except for very thin films. Nevertheless generally, magnetization in adjacent layers are tilted from the external magnetic field direction. This is called the twisted state ( $\mathrm{T}$ state). The magnetic state of the system is dependent on the ratio of the total magnetic moment of the layers $\left(m^{F e}: m^{G d}\right)$. Notice that this relation is thickness-dependent. Studies have been carried out in order to describe the relative orientation of the magnetization as a function of the thickness ratio of the layers ([Van Aken, 2004], see figure 15).

Studies have also found that with changes in temperature the prevalent magnetic state may switch. This is basically due to the Curie temperature of the $\operatorname{Gd}\left(T_{C}^{G d}\right)$, that is reported to be close to room temperature and because of the change of the ratio $M_{s}^{F e}: M_{s}^{G d}$, since the Gd saturation magnetization decreases very rapidly with the temperature, and therefore a change in the ratio $m^{F e}: m^{G d}$ occurs. In this work we have studied both variables, since we characterize different samples with different thickness ratios but also work at different tem- 


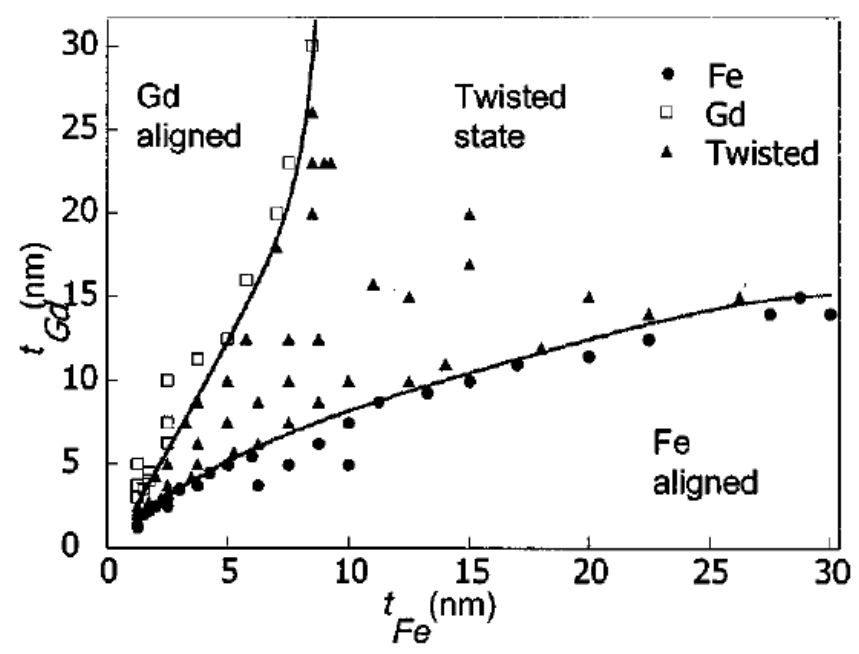

FIG. 15: Diagram of the magnetic states as a function of the Fe layer thickness (bottom axis) and the Gd layer thickness (left axis). The drawn lines indicate the boundaries between the G (open squares), the twisted (triangles) and the F (circles) states. The corresponding layer thicknesses are given on the top, [Van Aken, 2004]

peratures, what changes the total magnetization ratio through the change in the saturation magnetization of Gd.

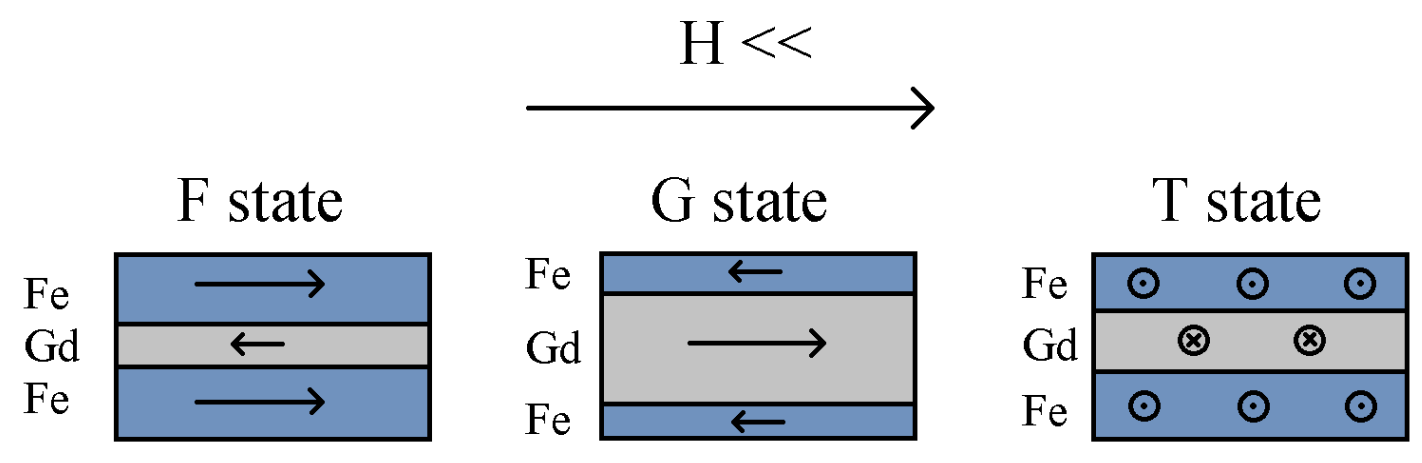

FIG. 16: Schematics of the different aligment states of the Fe/Gd multilayers at weak fields. Magnetization direction is depicted by arrows. 


\section{A. Ferromagnetic Resonance}

Ferromagnetic resonance (FMR) is a well known technique for the characterization of magnetic systems. As stated in Chapter II, section D., at a certain external static magnetic field $\left(H_{D C}\right)$ and under the application of a RF signal of frequency $f$, the magnetic sample starts absorbing power. As $H_{D C}$ is swept the magnetization will begin to resonantly precess and will therefore absorb energy. The static magnetic field of maximum absorption is called the resonance field $\left(H_{r e s}\right)$. This is the resonance condition and is dependent on the shape of the specimen. In the particular case of thin films, that relation can be analytically calculated. The derivation of the resonance condition on a thin film was made by [Kittel, 1947] and is:

$$
f=\frac{\gamma \mu_{0}}{2 \pi} \sqrt{H_{\text {res }}\left(H_{\text {res }}+M_{s}\right)}
$$

FMR consists in the measurement of the power absorbed by the sample at every resonance condition, associating each frequency with a resonance field in order to obtain the relation $f(H)$. Due the setup experiment, the DC voltage measured represents the derivative of the absorbed power as a function of the external magnetic field, whose equation is:

$$
\frac{d P}{d H_{D C}}=K_{1} \frac{4 \Delta H\left(H-H_{r e s}\right)}{\left[4\left(H-H_{r e s}\right)^{2}+(\Delta H)^{2}\right]^{2}}-K_{2} \frac{(\Delta H)^{2}-4\left(H-H_{r e s}\right)^{2}}{\left[4\left(H-H_{r e s}\right)^{2}+(\Delta H)^{2}\right]^{2}}+\text { slope }+ \text { off set }
$$

After the FMR measurements, it is possible to fit the data to a curve of the form $\frac{d P}{d H_{D C}}$, by optimizing the parameters $H_{\text {res }}, \Delta H, K_{1}, K_{2}$, offset and slope. Figure 17 shows an example of one of our multilayers, whose spectra has been studied.

Once the condition of resonance has been obtained for different frequencies, we display $H_{r e s}$ as a function of the frequency (Kittel curve), which allows to extract the saturation magnetization out of the fitting curve of the Kittel relation. In figure 18 we can see the relation between the frequency and $H_{\text {Res }}$ in an example.

The field linewidth $\Delta H$ is defined as the full width at half amplitude of the absorption curve. There are two main contributions to the linewidth : (I) the intrinsic linewidth, due to damping, is a fundamental property of the material and (II) the extrinsic linewidth is 


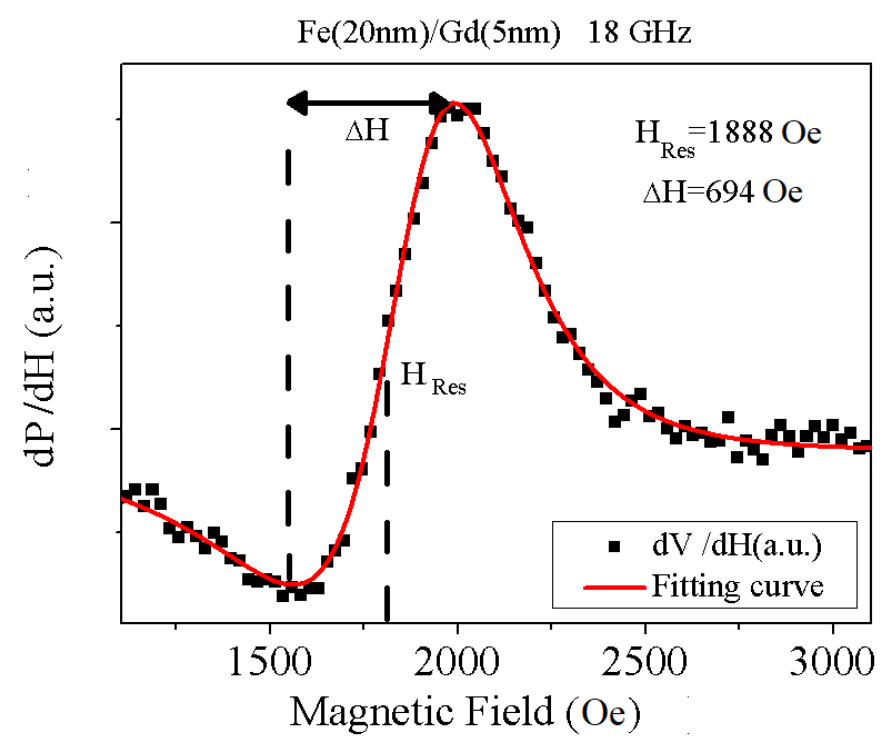

FIG. 17: Example of $d P / d H_{D C}$ measured in a multilayer of $\left.\mathrm{Fe}(20 \mathrm{~nm}) / \mathrm{Gd}(5 \mathrm{~nm})\right]_{5}$ at $18 \mathrm{GHz}$

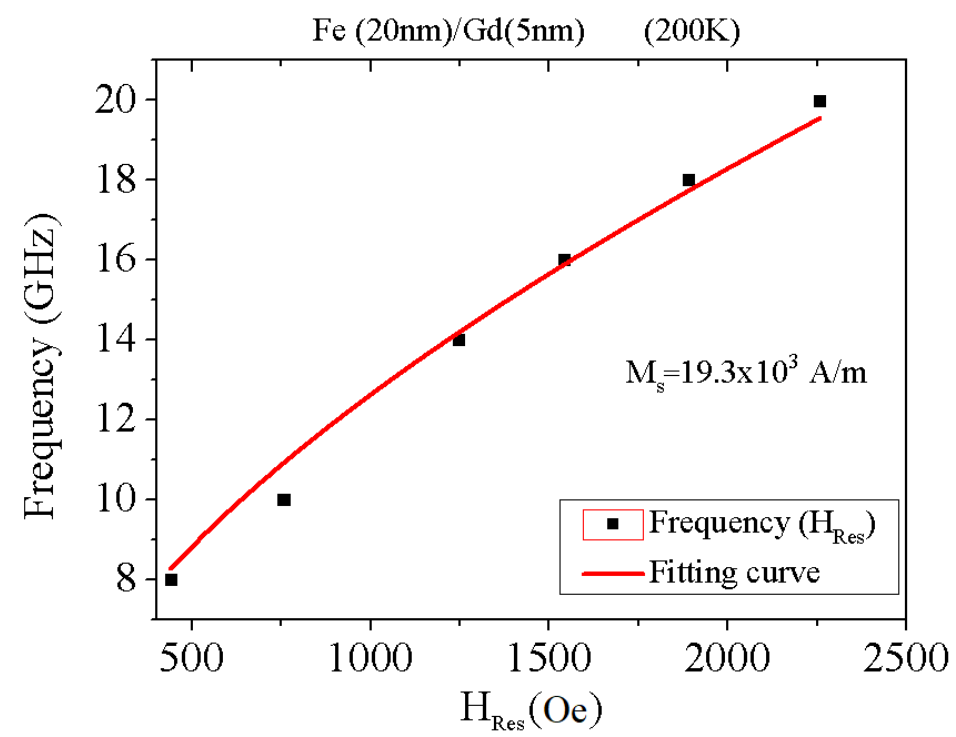

FIG. 18: Kittel Curve fitted obtained from resonance fields $H_{\text {Res }}$ measured at different frequencies.

associated with magnetic inhomogeneities within the sample and the anisotropy dispersion in the film. The interest in FMR linewidth is due in part to the role the damping phenomenon plays in spintronic devices. For a simple system such as a single thin film and when using the absorption derivative curve, the intrinsic linewidth has a linear variation with the frequency and is given (for a saturated sample) by : 


$$
\Delta H=\frac{2 \omega \alpha}{\sqrt{3} \gamma}
$$

where $\omega$ is the angular frequency, $\gamma$ is the gyromagnetic ratio and $\alpha$ the damping constant.

Fe/Gd multilayer samples are grown in the ISOM facilities, deposited by sputtering method. In order to control their thickness we used a controlled- by-software stepper motor that allowed the control of the sample holder inside the sputtering chamber with a precission in time of $0.1 \mathrm{~s}$ and angle precission of $1^{\circ}$. Samples have the following structure : Substrate/Cr $(4 \mathrm{~nm}) / F e\left(t_{F e} \mathrm{~nm}\right) / G d\left(t_{G d} \mathrm{~nm}\right)(\mathrm{x} 5) / F e\left(t_{F e} \mathrm{~nm}\right) / \operatorname{Pt}(2 \mathrm{~nm})$. FMR measurements where performed in a Physical Proeperty Measurment System (PPMS) in the CNRS/THALES facilities in Palaiseau (France). 


\section{B. Fe (20)/ Gd (5) Multilayer Sample.}

We present the FMR results of a sample whose structure is: Substrate/Cr(4)/[Fe(20)/Gd(5) $]_{5} / \mathrm{Fe}(20) / \mathrm{Pt}(2)$. We perform FMR measurements at a range of temperature that goes from $300 \mathrm{~K}$ to $50 \mathrm{~K}$. In this structure the width of the $\mathrm{Fe}$ layers are thicker, therefore, measurements are carried out in a range of frequencies (10 $\mathrm{GHz}-20 \mathrm{GHz}$ ) suitable for this material. Fe/Gd multilayers that fulfill the condition $m^{F e}>m^{G d}$ present a $\mathrm{F}$ state, which are characterized by the alignment at weak magnetic fields of magnetic moments of the Fe layers and the external magnetic field, whereas the magnetic moments in Gd layers align opposite to the external $H$. Figure 19 plots FMR curves at $f=18 \mathrm{GHz}$ for different temperatures.

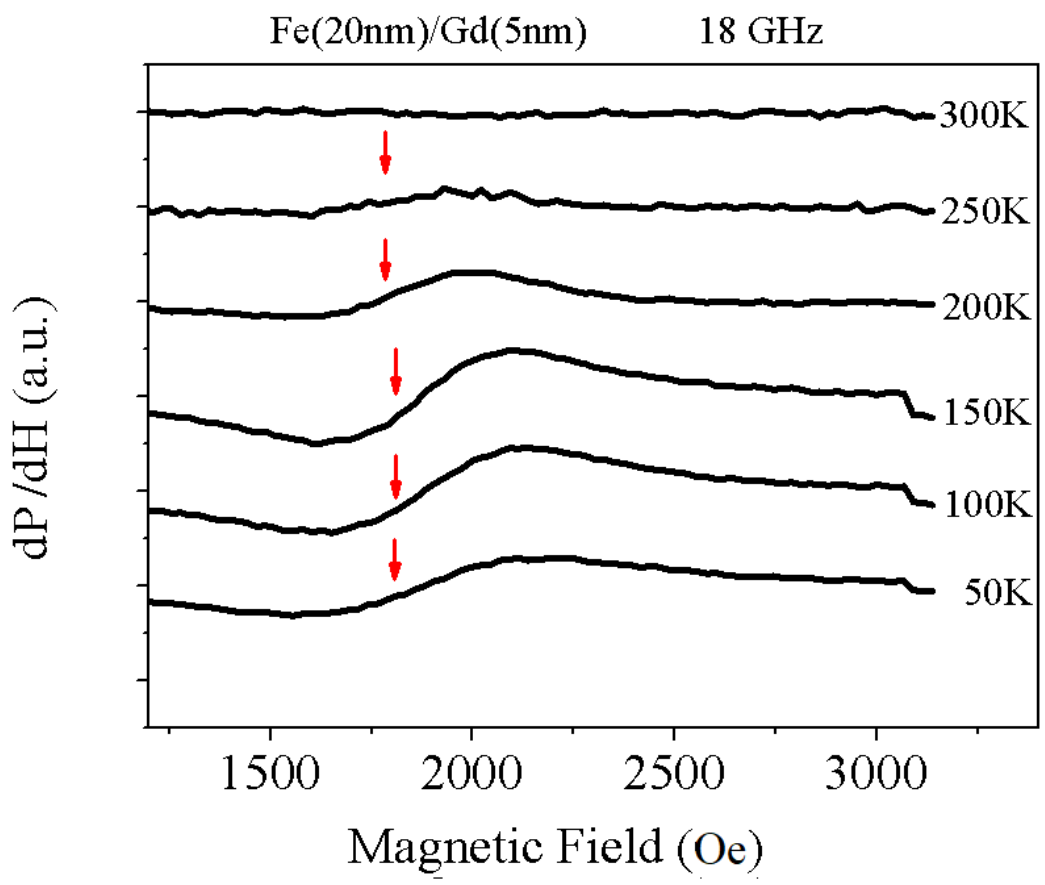

FIG. 19: Resonance spectra at $f=18 \mathrm{GHz}$ and different temperatures $(50-300 \mathrm{~K})$ shown in the plot. $H_{r e s}$ at every temperature is marked by arrows.

$H_{\text {res }}$ is marked by red arrows at every temperature. At $300 \mathrm{~K}$ this sample did not show any visible resonance at a frequency of $18 \mathrm{GHz}$. Nevertheless, the resonance condition is achieved at lower temperatures. For the sake of clarity, we present a comparison of the resonance spectra at two different temperatures $(300 \mathrm{~K}$ and $50 \mathrm{~K}$ ) with the same offset in figure 20 . 


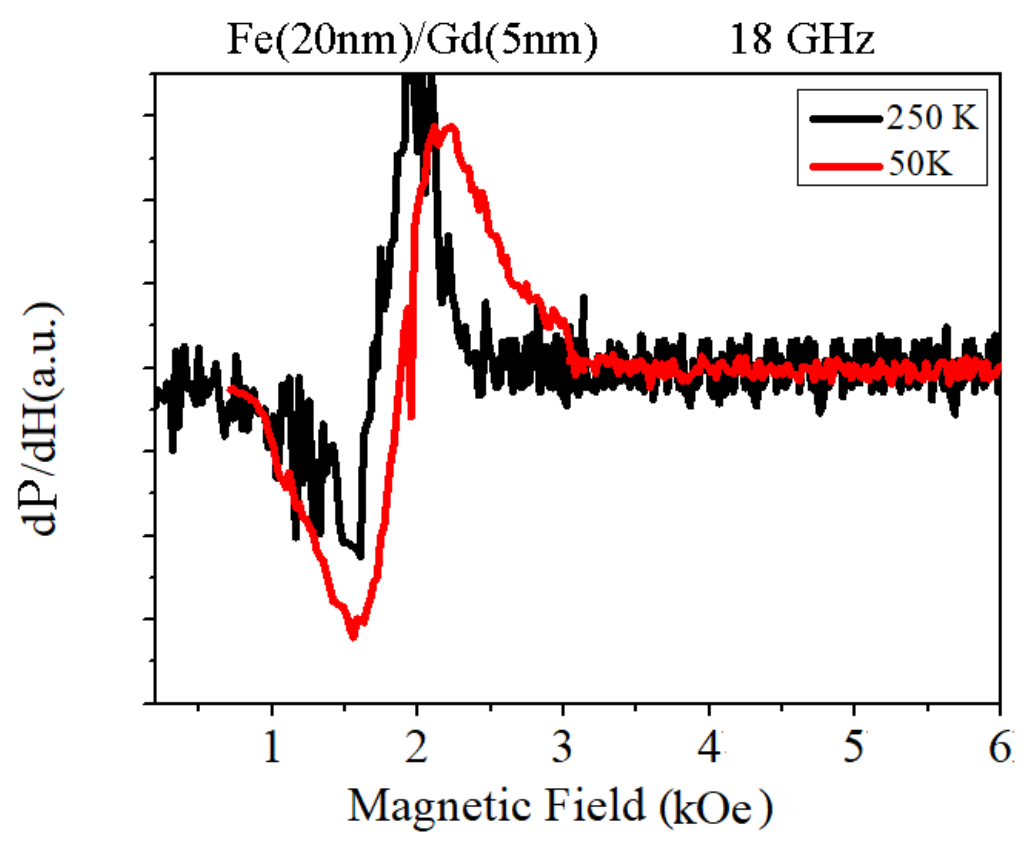

FIG. 20: Comparison of the spectra of the sample $\mathrm{Fe}(20 \mathrm{~nm}) / \mathrm{Gd}(5 \mathrm{~nm})$ at $f=18 \mathrm{GHz}$ at $250 \mathrm{~K}$ and $50 \mathrm{~K}$.

We note that $H_{\text {Res }}$ varies little with the temperature $(T)$ for this sample and without any tendency as a function of $T$ (figure 21 a) ). On the other hand, as the temperature decreases the resonance peak broadens. The resulting is a temperature dependency of the linewidth $\Delta H(T)$ as shown in figure $21 \mathrm{~b})$
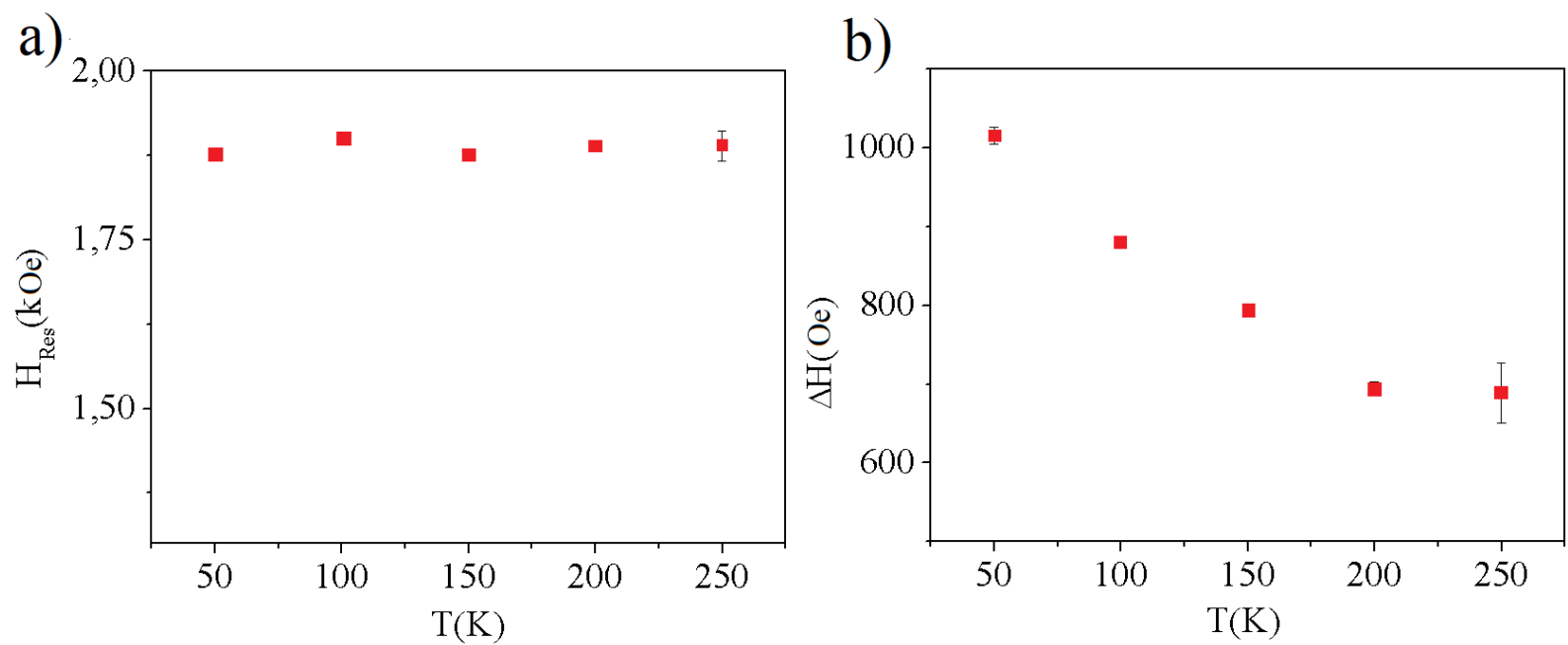

FIG. 21: a) Resonance field and b) linewidth as a function of temperature at $f=18 \mathrm{GHz}$ for a supperlatice of structure $[\mathrm{Fe}(20) / \mathrm{Gd}(5)]_{5}$. 
$H_{\text {Res }}$ is related through the equation (6) to the saturation magnetization of the sample, therefore, this remains the same at all temperatures. In the case of the linewidths, it is linearly correlated with the damping parameter $\alpha$ through equation (10), so these results show how $\alpha$ increases as the temperature decreases. The resonance fields remain similar for all temperatures at every frequency, which can be observed by comparing the Kittel curves. In figure 22 we compare the Kittel curves fitted from the data at $300 \mathrm{~K}$ and $50 \mathrm{~K}$. All fitted curves in between at different temperatures, $\mathrm{T}=[250 \mathrm{~K}, 200 \mathrm{~K}, 150 \mathrm{~K}, 100 \mathrm{~K}]$ present similar values of $M_{s}$. We can conclude that at all temperatures the same resonace mode is present, which is associated with the $\mathrm{F}$ state, where the magnetization in the Fe layer aligns with $H$.
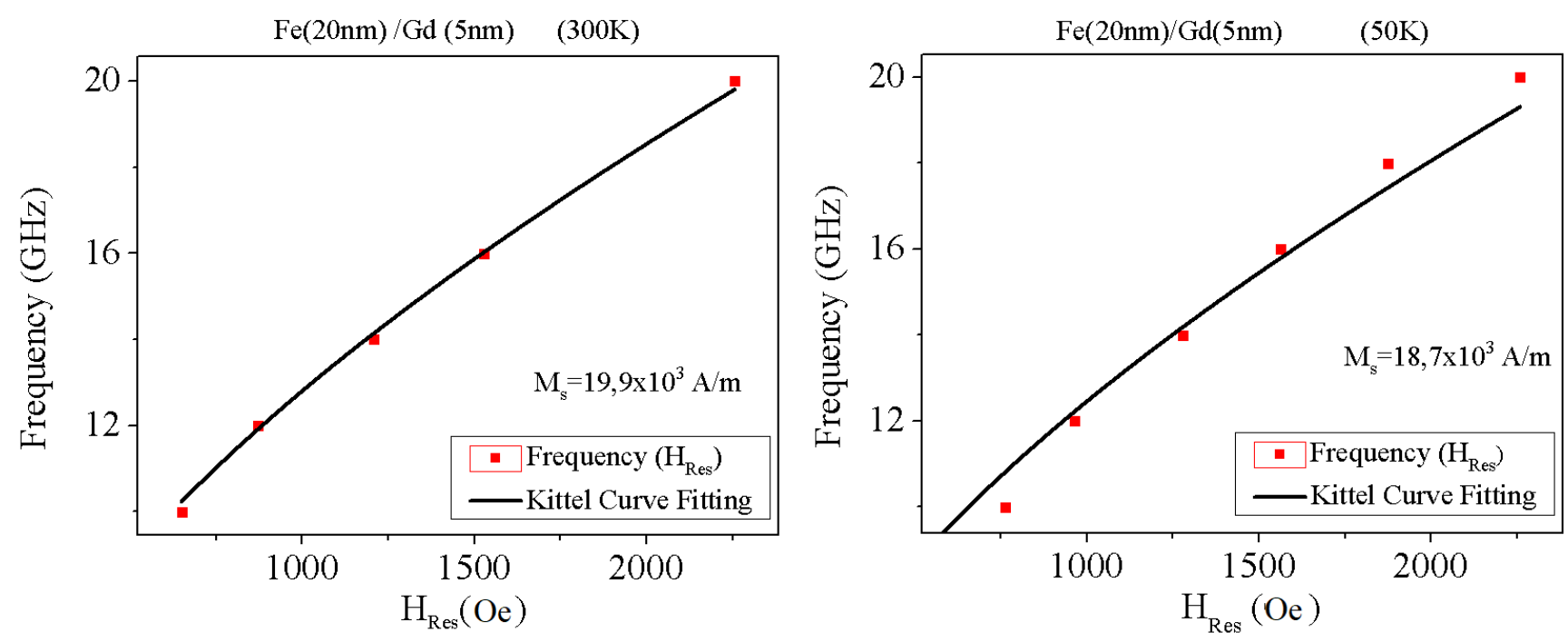

FIG. 22: Plot of the $H_{\text {Res }}$ as a function of the frequency. Fitting curves of the Kittel equation are represented over the data.

By computing the best approximation for the Kittel curve for $H_{R e s}$ at every temperature, the saturation magnetization can be calculated as a parameter. At all temperatures $M_{s}$ remains within a narrow range. The gyromagnetic factor used in the computations is $\gamma / 2 \pi=2.94(\mathrm{GHz} / \mathrm{kOe})$. As stated before, with this thickness ratio between Fe and Gd layers, the $\mathrm{F}$ phase is expected to be found. Therefore these results of $H_{\text {Res }}$ and $M_{s}$ can be associated to the $\mathrm{F}$ state. As no significant change is observed in the results at different temperatures, we conclude that the $\mathrm{F}$ state is always prevalent in this sample and the $\mathrm{Fe}$ layers align with the external magnetic field, so only one mode is observed. 


\section{Fe (5)/ Gd (15) Multilayer Sample.}

We now describe FMR measurements of samples whose structure is: Substrate/ $\mathrm{Cr}(4) /[\mathrm{Fe}(5) / \mathrm{Gd}(15)]_{5} / \mathrm{Fe}(5) / \mathrm{Pt}(2)$. This structure is expected to present a $\mathrm{G}$ alignment state upon a weak external magnetic field, due to the larger thickness of the Gd layers. In this state, the Gd layers aling with $H$, whereas the Fe layers align opposite to $H$ because of the AFM coupling in the interlayer. Nevertheless at high fields this sample it is expected to present a twisted state. The Curie temperature of the $\mathrm{Gd}$ is $T_{c}^{G d}=293 \mathrm{~K}$, therefore, a transition is expected to occur at this temperature. However, different works, e.g. [Hosoito, 2012], reported that in this type of samples, G state is present for lower temperatures $T<120 K$ and $\mathrm{F}$ state prevails for higher temperatures $T \geq 140 K$. Spectra at different temperatures at a frequency of $18 \mathrm{GHz}$ is shown in figure 23 .

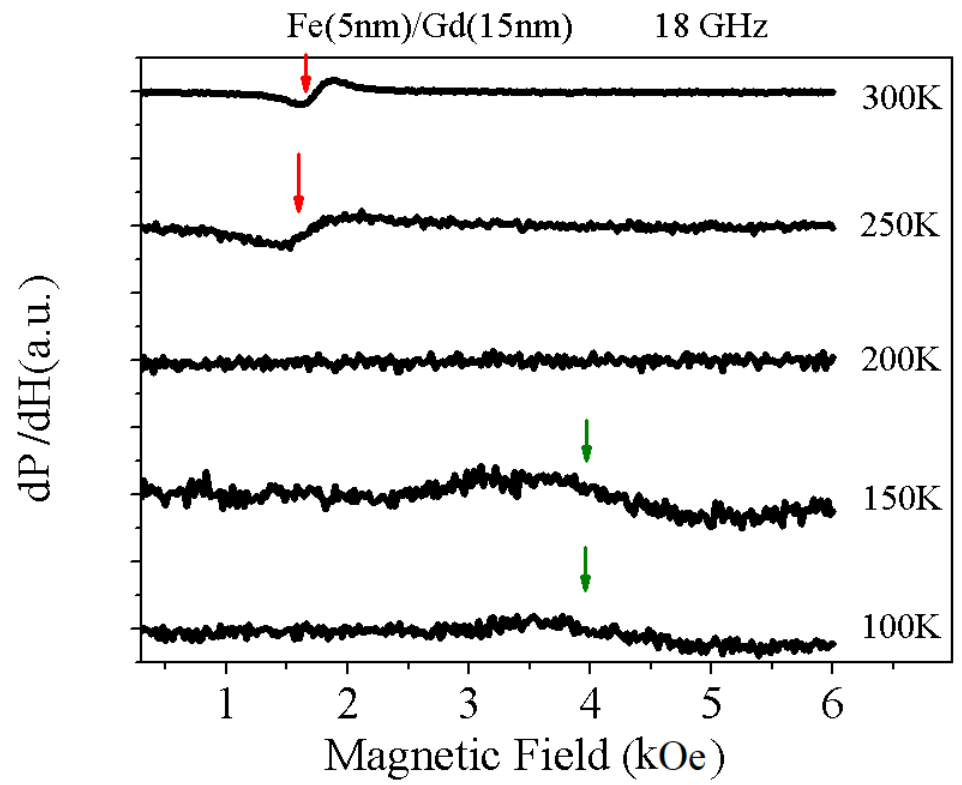

FIG. 23: Resonance spectra at $f=18 \mathrm{GHz}$ and different temperatures $(100-300 \mathrm{~K})$. $H_{\text {res }}$ of the first and second resonance modes are marked by red and green arrows respectively.

At $300 \mathrm{~K}$ and $250 \mathrm{~K}$ the sample presents a resonance at 1737 Oe and 1607 Oe. This mode is related with the one seen in the previous sample, which is associated with the F state. At $250 \mathrm{~K}, H_{R e s}$ shifts to lower fields, plus the resonance peak $\Delta H$ broadens with respect to $300 \mathrm{~K}$. The same behaviour was observed earlier for different types of TM/Gd multilayers ([Partin, 2006], [Demirtas, 2010]). This effect can be qualitatively described, considering a 
strongly coupled layered ferrimagnet, so this behaviour can be considered as normal. We can observe this at all frequencies for this mode in this sample, as shown in figure 24 .

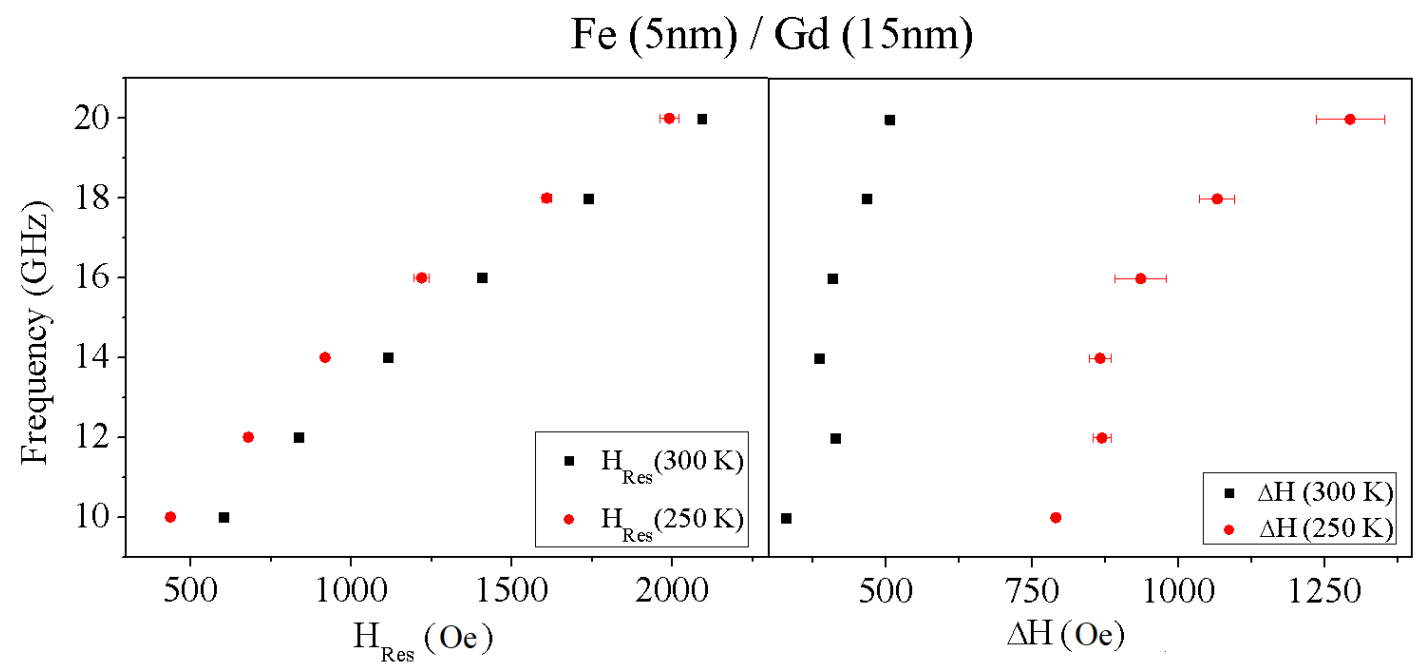

FIG. 24: a) Comparison of the first resonance mode at $300 \mathrm{~K}$ and at $250 \mathrm{~K}$. b) Comparison of $\Delta H$ at $300 \mathrm{~K}$ and at $250 \mathrm{~K}$ for all frequencies

At the temperature of $200 \mathrm{~K}$ we observe no resonance at any frequency. This indicates that a transition occurs in the sample. At $150 \mathrm{~K}$ a second resonance appears, which corresponds to another resonance mode (figure 23 marked by green arrows). The resonance fields of this second mode are present at higher fields, with a larger linewidth $\Delta H$ than the first resonance mode. We address the first mode as the high temperature mode $(\mathrm{H}-\mathrm{T})$, whereas we address the second mode as the low temperature mode (L-T). We display a close up of the comparison of the resonances at $300 \mathrm{~K}$ and $100 \mathrm{~K}$ in figure 25

At all frequencies under study, the H-T mode disappears below $T=250 \mathrm{~K}$. The activation of the $\mathrm{L}-\mathrm{T}$ mode below $200 \mathrm{~K}$ is caused by the transition from paramagnetic to ferromagnetic in the Gd. The reported Curie temperature of the $\mathrm{Gd}$ is $T_{C}^{G d}=293 \mathrm{~K}$, however, it seems that the system needs the temperature to be further decreased in order to reach the compensation point $\left(T_{\text {comp }}\right)$, where the magnetic moments in the Fe layers are comparable to the magnetic moments in the Gd layers $\left(m^{F e} \sim m^{G d}\right)$. The resonant fields of the L-T mode appear in the region of high fields. Previous works have shown that, for similar structures, at magnetic fields this high, Fe-Gd multilayers present a twisted state, 


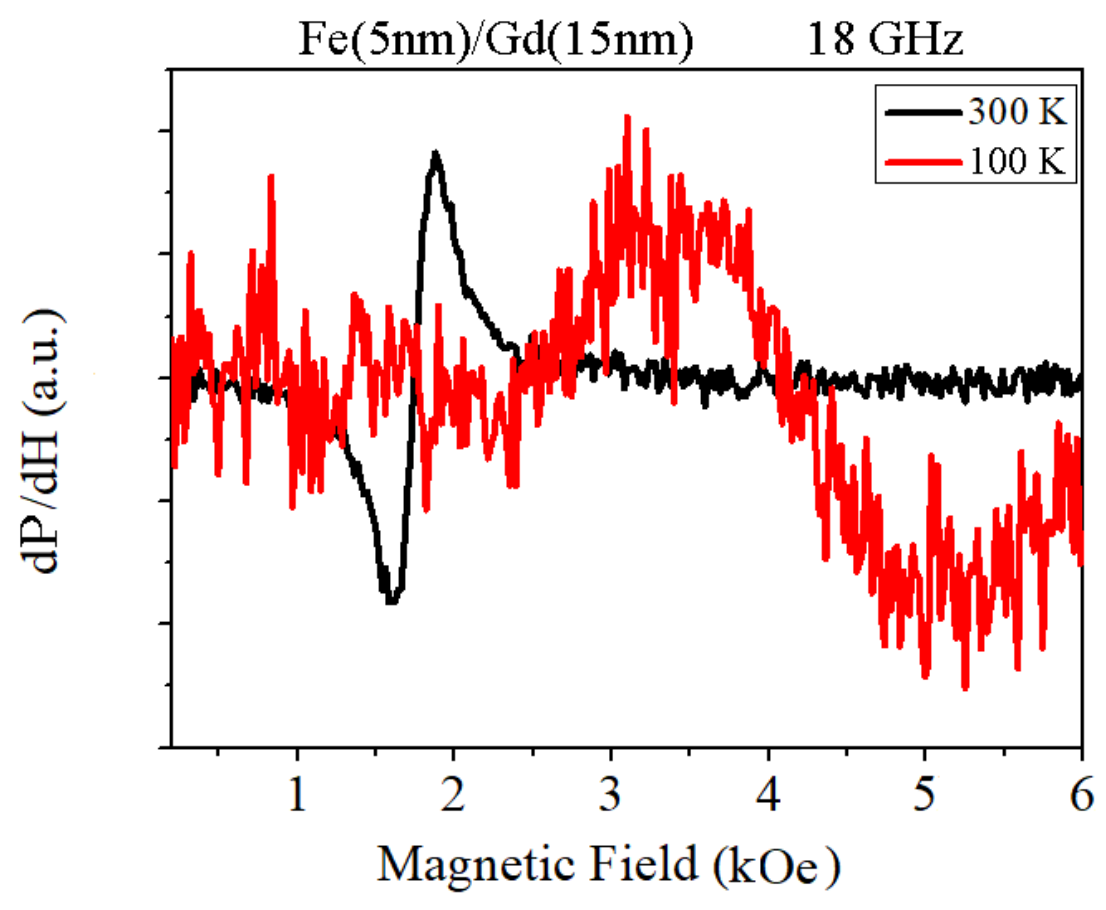

FIG. 25: Comparison of the resonance spectra at $18 \mathrm{GHz}$ of the $\mathrm{Fe}(5 \mathrm{~nm}) / \mathrm{Gd}(15 \mathrm{~nm})$ at $300 \mathrm{~K}$ (black curve) and at 100K (red curve). First resonance mode are marked by red arrows and a second resonance mode is marked by green arrows.

which is observed by a smooth transition in the measurements of the magnetic moment [Drovosekov, 2017]. We measure these modes for a range of frequencies as in the previous case so both modes can be compared and we plot $f(H)$, as shown in figure 26 .

At room temperature the $\mathrm{f}(\mathrm{H})$ relation for the high-temperature mode follows qualitatively the Kittel formula for a ferromagnetic thin film. However at lower temperatures the shape of the $\mathrm{f}(\mathrm{H})$ curve changes dramatically and the Kittel's formula becomes inapplicable. This agrees with the the model presented by [Drovosekov, 2019], which predicts the existence of two spectral branches with different types of magnetic precession inside Gd layers. Therefore, in the L-T mode the approximation of uniform magnetization precession within the structure is not valid. Taking into account a large exchange stiffness of Fe layers and a strong coupling at Fe-Gd interface, we can suppose that inhomogeneous precession occurs inside the Gd layers. We conclude that two modes are observable in this sample, a first mode at higher temperatures that is related to a collinear state that vanishes below $250 \mathrm{~K}$ and a second mode associated with a twisted state, that appears below $200 \mathrm{~K}$. 


\section{$\mathrm{Fe}(5 \mathrm{~nm}) / \mathrm{Gd}(15 \mathrm{~nm})$}

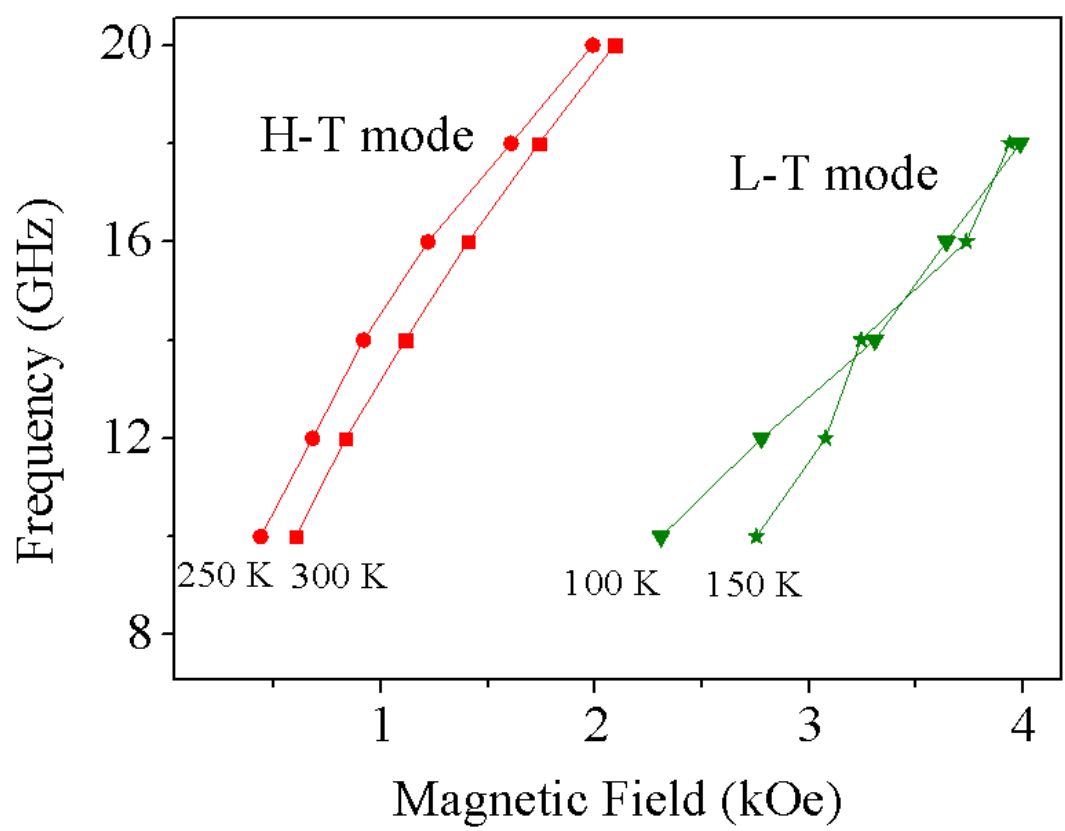

FIG. 26: Resonance frequency as a fuction of applied field $\mathrm{f}(\mathrm{H})$, at different temperatures for a sample whose structure is $[\mathrm{Fe}(5) / \mathrm{Gd}(15)]_{5} / \mathrm{Fe}(5) / \mathrm{Pt}(2)$. Two modes are observed, H-T mode, which is related to a collinear $\mathrm{F}$ state and follows the Kittel relation for a thin film and L-T mode associated with a twisted state, which does not follow the Kittel relation.

\section{Fe (10)/ Gd (10) Multilayer Sample.}

We perform FMR measurements in a sample whose structure is: Substrate $/ \mathrm{Cr}(4) /[\mathrm{Fe}(10) / \mathrm{Gd}(10)]_{5} / \mathrm{Fe}(10) / \mathrm{Pt}(2)$. Samples with this structure are expected to present a fundamental twisted state. In the twisted state, no layer is completely aligned with the external magnetic field, instead, both set of layers are tilted away from this preferential direction. This state is a compromise between the minimation of Zeeman energy, (because none of the magnetic moments dominates $m^{F e} \sim m^{G d}$ ) and the AFM coupling that prevails in the interlayer, where the moments of adjacent layers present opposite magnetization directions. We present the FMR measurements for $18 \mathrm{GHz}$ frequency at different temperatures in figure 27. 


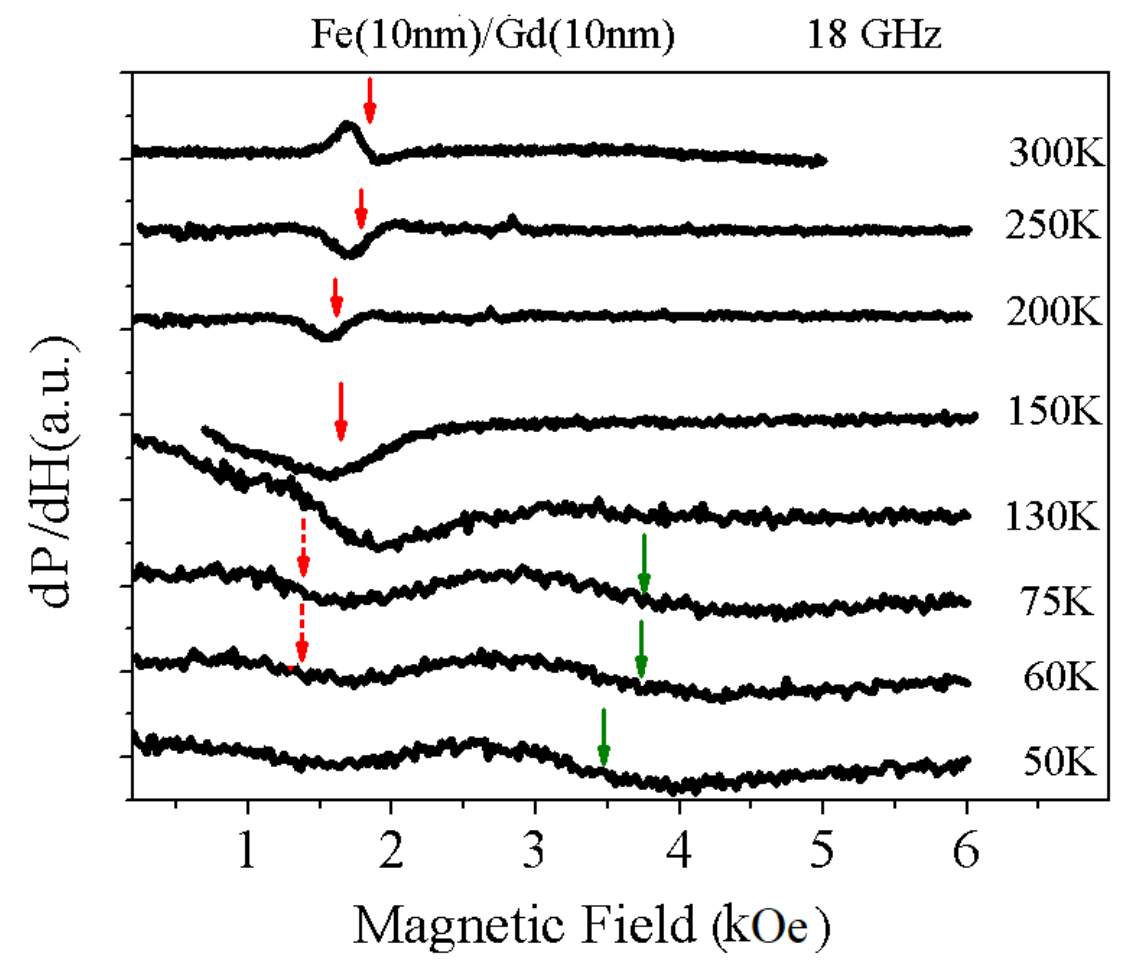

FIG. 27: Resonance spectra at $f=18 \mathrm{GHz}$ and different temperatures (50- $300 \mathrm{~K}$ ) shown in the plot. Two resonance modes are shown by arrows.

From $300 \mathrm{~K}$ down to $150 \mathrm{~K}$, only one resonance is visible, whose resonance conditions match the ones in the H-T mode found in the previous samples, which is associated to a collinear state. As the temperature decreases from room temperature, the resonances of the $\mathrm{H}-\mathrm{T}$ mode shift towards lower resonance fields with larger linewidth $(\Delta H)$. Below $130 \mathrm{~K}$ a second mode (L-T), starts to appear. At frequencies above $16 \mathrm{GHz}$, the $\mathrm{H}-\mathrm{T}$ mode is present even at low temperatures (marked by dashed red arrows in figure 27), however, most of the resonance fields of the $\mathrm{H}-\mathrm{T}$ mode at low temperatures are impossible to compute due to the proximity of the L-T mode. In figure 28 , we display a comparison of the spectra $(f=18$ $\mathrm{GHz}$ ) at $250 \mathrm{~K}$ and $60 \mathrm{~K}$.

Figure 28 show that the $\mathrm{H}-\mathrm{T}$ mode is obviously present at $250 \mathrm{~K}$, nevertheless it is also present at low temperatures, where coexist with the L-T mode. This only occurs at frequencies above $16 \mathrm{GHz}$, since below that frequency the $\mathrm{H}-\mathrm{T}$ mode is not observed at low temperatures. This is clearly different from the previous sample, where the H-T mode vanishes below $\mathrm{T}=250 \mathrm{~K}$. Since we have associated the $\mathrm{H}-\mathrm{T}$ mode with a collinear state and 


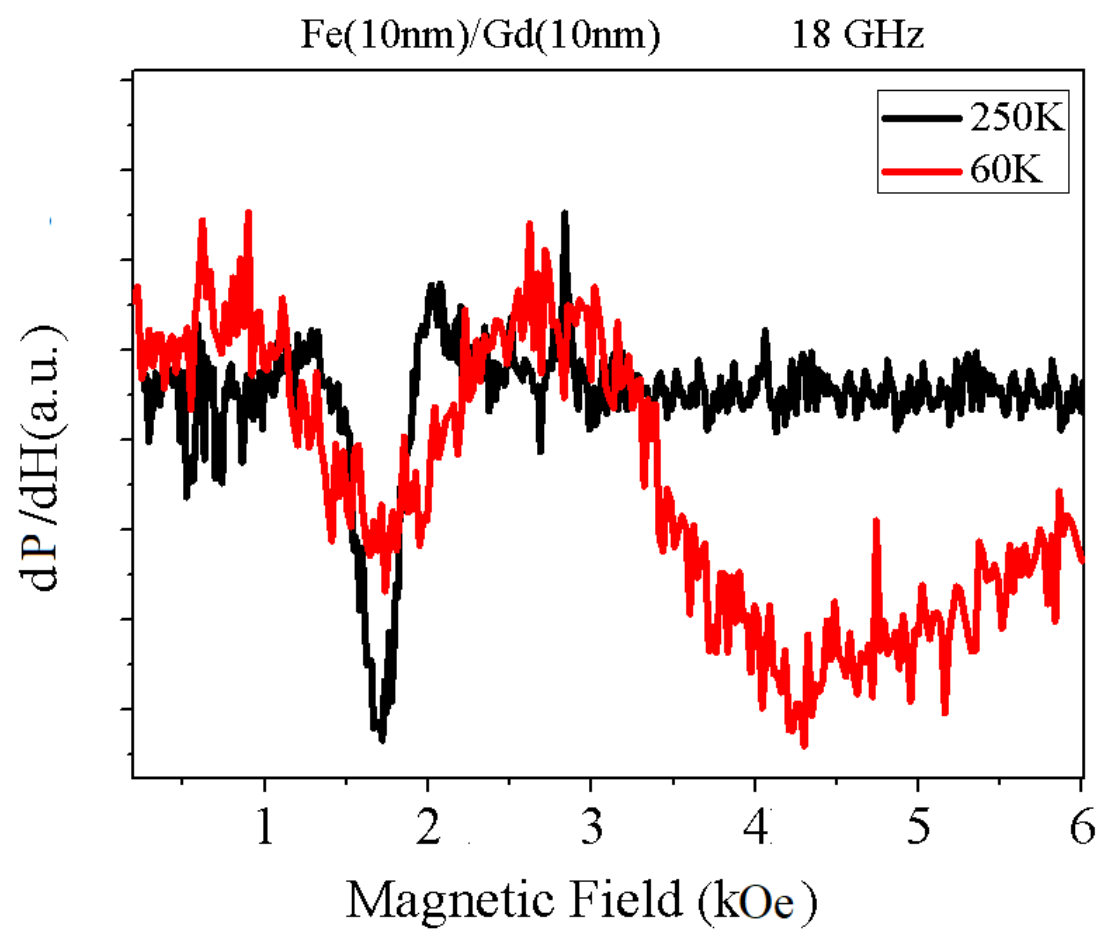

FIG. 28: Comparison of spectras at $f=18 \mathrm{GHz}$ for temperatures $\mathrm{T}=250 \mathrm{~K}$ with one mode and $\mathrm{T}=60 \mathrm{~K}$ two modes.

the L-T mode with a twisted state, it is possible that in this sample the FMR measurement show the transition between both alignment states, which according to [Drovosekov, 2017] for a similar sample, occurs above $1 \mathrm{kOe}$.

We now represent the $\mathrm{f}(\mathrm{H})$ relation in figure 29, where the resonance frequencies are displayed as a function of the applied field at different temperatures. For clarity purposes we only represent the resonance fields that are clearly calculated. The H-T mode can be qualitatively described by the Kittel formula for a thin film close to room temperature, however it is not applicable for the L-T mode. As the temperature decreases, the resonances of the H-T mode are only measurable at higher frequencies, (for instance, no resonance is observed at $150 \mathrm{~K}$, up to $16 \mathrm{GHz}$ ). This agrees with the model presented in [Drovosekov, 2019] for a $\mathrm{Fe} / \mathrm{Gd}$ multilayer, which predicts a gap in the $\mathrm{H}-\mathrm{T}$ mode close to the critical temperature, which is related to a strongly non-uniform precession inside the Gd layers. Finally we can conclude the observation of two resonance modes (H-T and L-T), associated with different alignment states and different precession profiles. 


\section{$\mathrm{Fe}(10 \mathrm{~nm}) / \mathrm{Gd}(10 \mathrm{~nm})$}

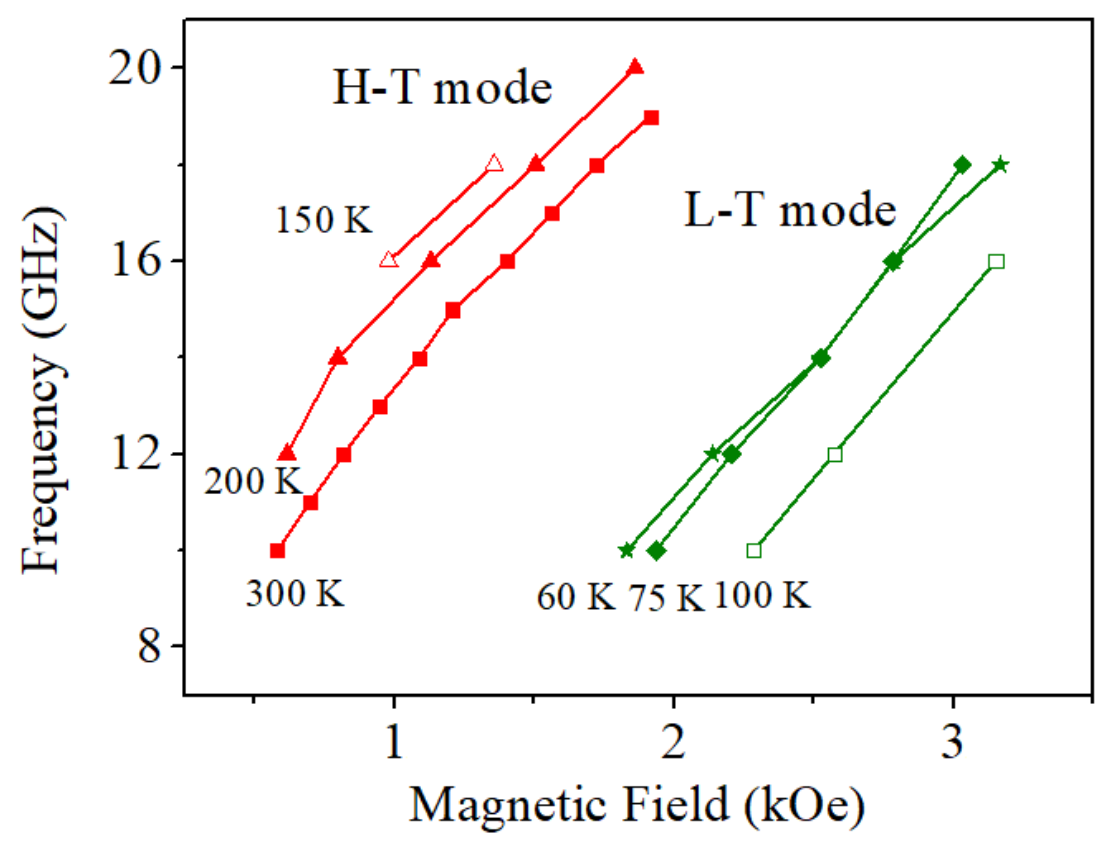

FIG. 29: Plots of the resonance fields as a function of the frequencies at high temperature with one mode, and at low temperatures with two simultaneous modes.

\section{E. Conclusions}

Ferromagnetic resonance (FMR) measurements are carried out in three Fe/Gd multilayers with different ratios of adjacent layers thicknesses. In these structures, the spins at the interlayers have an antiferromagnetic (AFM) coupling and present different states depending on which material aligns with the external magnetic field applied. The magnetic configuration depends on the ratio of the total magnetization between Fe and Gd. Modifying the temperature changes the ratio between the magnetization of the layers, which allows a multilayer sample to present different magnetic configurations at different temperatures.

Results of FMR measurements in a multilayer sample whose structure is $\mathrm{Cr}(4) /[\mathrm{Fe}(20) / \mathrm{Gd}(5)]_{5} / \mathrm{Fe}(20) / \mathrm{Pt}(2)$, show a defined resonance mode that is associated to a collinear state. The resonance condition is measured at different temperatures $(300 \mathrm{~K}, 250$ $\mathrm{K}, 200 \mathrm{~K}, 150 \mathrm{~K}$ and $100 \mathrm{~K}$ ), and for all of them the relation $f(H)$ is very similar, following the Kittel relation (6). It is expected that this sample shows a $\mathrm{F}$ aligned state, since the 
thickness of the Fe layers are larger than the Gd ones. Therefore the total magnetic moment of the Fe layers is larger at every temperature $\left(m^{F e}>m^{G d}\right)$, which forces a $\mathrm{F}$ aligned state.

Results of FMR measurements in the multilayer sample with structure $\mathrm{Cr}(4) /[\mathrm{Fe}(5) / \mathrm{Gd}(15)]_{5} / \mathrm{Fe}(15) / \mathrm{Pt}(2)$, show a resonance mode at high temperatures (H-T) $(300 \mathrm{~K}, 250 \mathrm{~K})$ which matches the one observed in the previous sample. This mode is associated with a collinear $\mathrm{F}$ state. In the vecinity of room temperature, the H-T mode shift towards lower resonance fields and larger linewidth with decreasing temperature. Due to the paramagnetic-ferromagnetic transition that the $\mathrm{Gd}$ undergoes at $T_{C}^{G d}=293 \mathrm{~K}$ and the larger thickness of the Gd sublayers in this sample, a tranisition is expected to occur. However this transition does not happen at $T_{C}^{G d}$, but at $\sim 200 \mathrm{~K}$ where no resonances are observed. At this point the magnetic moments in the Fe layers are comparable to the magnetic moments in the Gd layers $\left(m^{F e} \sim m^{G d}\right)$. Below that temperature a second mode $(\mathrm{L}-\mathrm{T})$ at low temperatures arises. This second mode appears in the region of the high fields, where a twisted state is expected in the sample. Although the H-T mode follows qualitatively the Kittel relation for a thin film the L-T mode does not, which is explained by an inhomogeneous precession of the magnetic moments inside the Gd layers

Finally we presented the results of FMR measurements in a multilayer sample whose structure is $\mathrm{Cr}(4) /[\mathrm{Fe}(10) / \mathrm{Gd}(10)]_{5} / \mathrm{Fe}(10) / \mathrm{Pt}(2)$. Similar to the sample whose Gd layer thickness was predominant, this sample presents two resonant modes at different range in temperatures (H-T and $\mathrm{L}-\mathrm{T})$, however at larger frequencies (16 and $18 \mathrm{GHz}$ ) and low temperatures, both modes can be observed in the same measurement. This may be due to the larger thickness of the Fe layers, which would imply that a collinear Fe state is present even at low temperatures at low fields, however, as the magnetic field is increased, a twisted state is reached in the multilayer and the second mode (L-T) can be observed. 


\section{MICROMAGNETIC SIMULATIONS ON NI NANOWIRES}

Nanowires are proposed to be the basic elements of new information storing devices in development. Since the patent of S. Parkin of the racetrack memory (RM), [Parkin, 2004], the study of the magnetization dynamics of these and analogous nanostructures has grown rapidly in the last decade. The main concept behind this memory is the sequential storage of the information in magnetic nanowires (NW's). The bits of information are magnetic domains separated by domain walls (DW's). In order to set the bit size, the DW's have to be pinned and depinned reliably at artificial defects placed at regular intervals throughout the magnetic NW. Here, we considered nickel (Ni) NW's where we have inserted a NiCu chemical defect with different Ni contents [Chen, 2006], [Matei, 2013]. One of the main reasons to use $\mathrm{NiCu}$ segments in the middle of a Ni NW is because of the simplicity of their fabrication process, furthermore, adding a non-magnetic element and/or segment to a magnetic NW has proved to be a good method to tailor the magnetic properties of such NW's [Chen, 2003], Carignan, 2007],[Susano, 2016]. In this simulation work the DW dynamics is studied in 50 $\mu m$-diameter cylindrical-shaped with a length of $1 \mu \mathrm{m}$. For a Ni nanocylinder with $50 \mathrm{~nm}$ in diameter, there is not an energetically favoured type of DW, and both transverse and vortex DW's can be present [Hertel, 2004]. This software used to simulate the DW dynamics is the object oriented micromagnetic framework (OOMF)[Donahue, 1999]. The software allows to mesh the space in cells of defined-by-user dimensions, define the saturation magnetization $\left(M_{s}=4.9 \cdot 10^{5} \mathrm{~A} / \mathrm{m}\right)$ at each cell, the exchange coupling $\left(A=9 \cdot 10^{-12} \mathrm{~J} / \mathrm{m}\right)$ and the anisotropy constants $\left(K=5.7 \cdot 10^{3} \mathrm{~J} / \mathrm{m}^{3}\right)$. The dimensions of the cell will be necessarily smaller than the characteristic exchange length $l_{e x}=\sqrt{A / \mu_{0} M_{s}^{2}}=7.72 \mathrm{~nm}$, which is the size of the smallest feature of interest, so that sampling at that length scale is sufficient to capture all relevant details [Abo, 2013]. For micromagnetics and other modeling involving details of magnetic domain formation and motion, the typical feature detail is the domain wall. The stopping condition chosen for the simulations was $|d m / d t|=1 \mathrm{deg} \cdot \mathrm{ns}^{-1}$ with a damping factor of 0.015 [Coey, 2009]. The initial conditions set the magnetic moment in each cell parallel to the long axis of the NW (z-direction), which is the easy axis of magnetization $\left(m_{0}=(0,0,-1)\right)$. In order to nucleate an initial vortex DW, the magnetic moments at one end of the NW are reversed $\left(m_{0}=(0,0,1)\right.$, figure $\left.30(\mathrm{~b})\right)$. For the transverse DW, the magnetic moments at the end of the NW are also reversed but forming a small angle with 
the NW axis as depicted in figure $30(\mathrm{~b})\left(m_{0}=(0.1,0.1,1)\right)$.

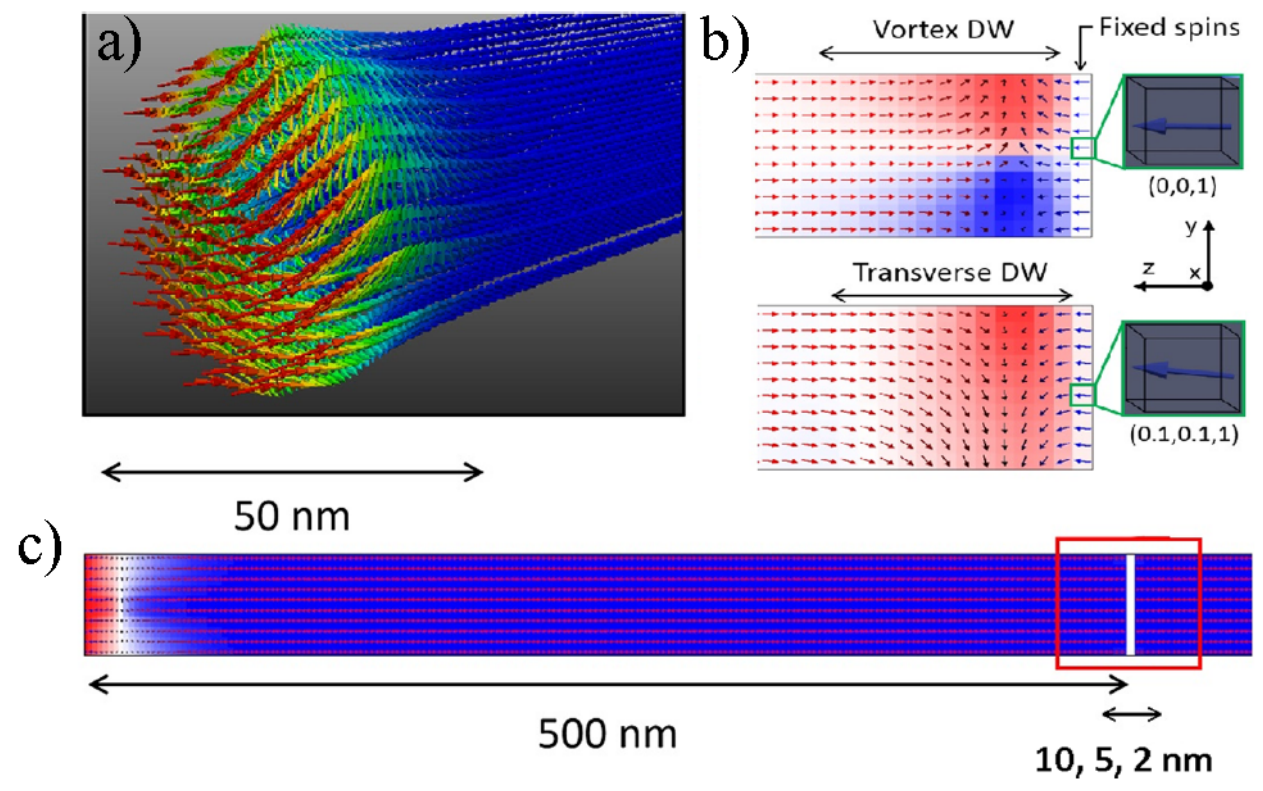

FIG. 30: (a) 3D schematic representation of a vortex domain wall nucleated at the edge of a cylindrical ferromagnetic nanowire (made with Paraview software). (b) Schematic representations of the nucleation process of a vortex and a transverse domain wall (DW). (c) 2D schematic representation of a cylindrical ferromagnetic nanowire with a chemical constraint [Castilla, 2017].

\section{A. Non-magnetic constraint}

First simulations consist in studying the dependence of the depinning field on the width of the chemical constraint when it is non-magnetic. We have used three different thicknesses for the constraint, 10, 5 and $2 \mathrm{~nm}$. The unit cell was $5 \times 5 \mathrm{~nm}^{2}$ in the $\mathrm{x}-\mathrm{y}$ plane, and 5 , 2.5 and $1 \mathrm{~nm}$ along the $\mathrm{z}$ axis, depending on the thickness of the chemical constraint under study (10, 5 and $2 \mathrm{~nm}$, respectively). We have checked that, by reducing the cell size along the $\mathrm{z}$ axis from 5 to $2.5 \mathrm{~nm}$, the simulations give only negligible differences $(<0.2 \%)$. First, we introduce a vortex DW at 0 Oe. This DW remains at one end of the wire until the applied external magnetic field is strong enough to depin it from the NW's end and it moves until it reaches the chemical constraint (figure 31). The propagation field has the same value $\left(H_{\text {prop }}=310 \mathrm{Oe}\right)$ regardless of the thickness of the constraint, as expected. As the DW moves through the NW, it keeps its vortex structure (figure 31 (a) and (b)). However, 
when the DW reaches the non-magnetic spacer, it gradually evolves to a transverse DW (figure 31 (c)). The transverse DW remains pinned at the spacer as the applied magnetic field increases, until it reaches a certain value $\left(H_{d e p}\right)$ at which the DW depins from the nonmagnetic constraint and moves towards the opposite end of the NW. After depinning, the DW moves forward with a mixed vortex-transverse structure, rotating as it travels. The depinning field increases with the thickness of the chemical constraint. The values obtained are 670,540 and 420 Oe for a 10,5 and $2 \mathrm{~nm}$ constraint, respectively.
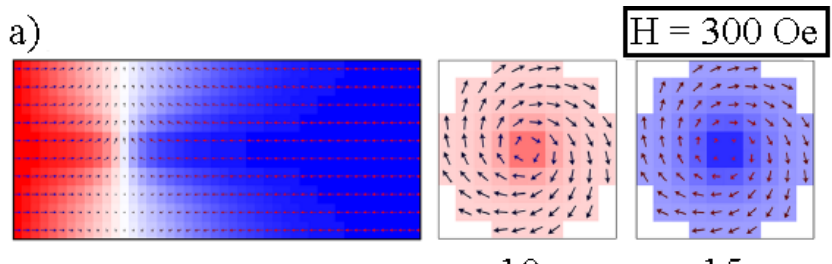

b)

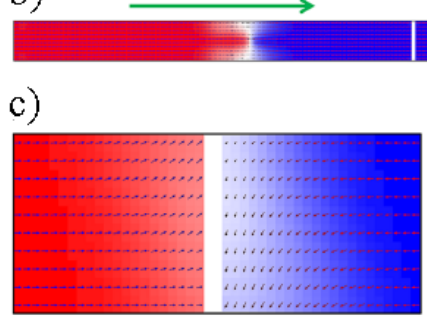

$\mathrm{z}=10 \mathrm{~nm}$

$\mathrm{H}=310 \mathrm{Oe}$

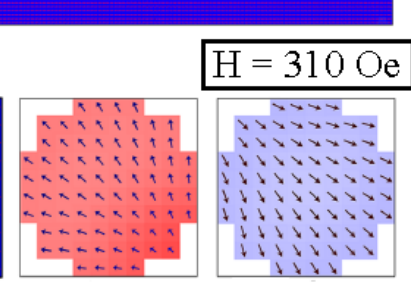

$\mathrm{z}=490 \mathrm{~nm} \quad \mathrm{z}=510 \mathrm{~nm}$ d)

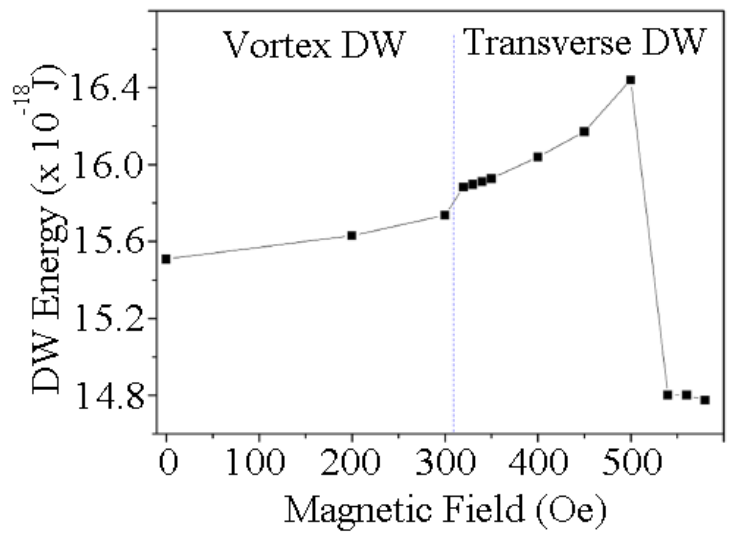

FIG. 31: 2D schematic representations of (a) a vortex domain wall nucleated at the left end of the nanowire; (b) a vortex domain wall moving though the cylindrical nanowire; and (c) the reconfigured transverse domain wall pinned at the $5 \mathrm{~nm}$-thick non-magnetic constraint. (d) Change in the domain wall energy with the applied magnetic field.

\section{B. Paramagnetic constraint}

In this section, the chemical constraint is simulated to be of paramagnetic $\mathrm{NiCu}$. The exchange coupling between the magnetic moments of adjacent cells in the chemical constraint is ignored. The initial nucleated domain wall is again a vortex. Table II summarizes the magnetic moments used for the simulations for the $\mathrm{NiCu}$ alloy with different $\mathrm{Ni}$ percentages25. Note that these are values for bulk. The column with exchange energy can be ignored for this section since $\mathrm{NiCu}$ is considered paramagnetic. Cubic cells of $5 \times 5 \times 5$ 
$\mathrm{nm}^{3}$ are used, smaller than the minimum exchange length for $100 \% \mathrm{Ni}$. In a paramagnetic chemical constraint, the vortex $\mathrm{DW}$ gets pinned at the $\mathrm{NiCu}$, only below $80 \% \mathrm{Ni}$ content (table III). The structure of the DW during the pinning process is the same as the one in the non-magnetic constraint. The injected vortex DW transforms to a transversal DW as it gets pinned at the constraint and it gets depinned in a mixed vortex-transversal structure with a non-centred axis.

\begin{tabular}{|c|cccc||}
\hline$N i(\%)$ & $T_{C}(K)$ & $M_{s}(k A / m)$ & $A(p J / m)$ & $l_{e x}(n m)$ \\
\hline \hline 90 & 529.51 & 411.80 & 7.58 & 8.44 \\
80 & 418.12 & 320.64 & 5.97 & 9.61 \\
75 & 362.28 & 275.67 & 5.17 & 10.41 \\
70 & 306.53 & 231.10 & 4.37 & 11.41 \\
65 & 250.79 & 186.94 & 3.57 & 12.75 \\
60 & 195.04 & 143.17 & 2.77 & 14.67 \\
55 & 139.30 & 99.81 & 1.98 & 17.78 \\
52 & 105.85 & 74.00 & 1.50 & 20.88 \\
51 & 94.70 & 65.42 & 1.39 & 22.74 \\
50 & 83.55 & 56.86 & 1.20 & 24.30 \\
\hline
\end{tabular}

TABLE II: Variation of the Curie temperature $\left(T_{C}\right)$, saturation magnetization $(\mathrm{Ms})$, exchange energy $(A)$ and magnetostatic, exchange length $\left(l_{e x}\right)$ with the $\mathrm{Ni} \%$ in a $\mathrm{NiCu}$ alloy.

The value of the depinning field for a paramagnetic $\mathrm{NiCu}$ constraint, depends only slightly on the Ni content. For the maximum possible Ni content that pins the DW (85\%), the depinning field is 440 Oe (table III). This value increases as the Ni content decreases, towards the value obtained for a $5 \mathrm{~nm}$ non-magnetic constraint (540 Oe). The domain wall energy during the pinning process is plotted in figure 4. Since there is no exchange energy in the constraint like in the case of the non-magnetic defect, the plot is very similar to the one shown in figure $2(\mathrm{~d})$. Indeed, the non-magnetic constraint is a particular case of the paramagnetic $\mathrm{NiCu}$ defect, with $0 \% \mathrm{Ni}$ content. 
a)

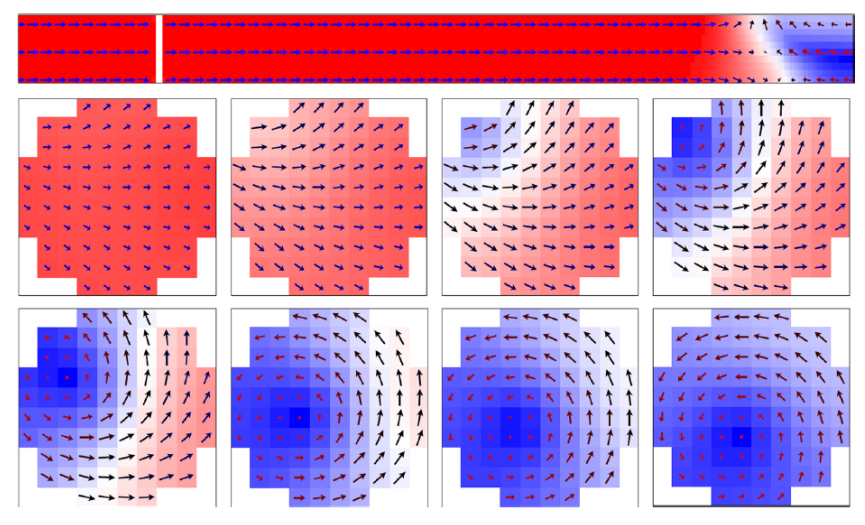

b)

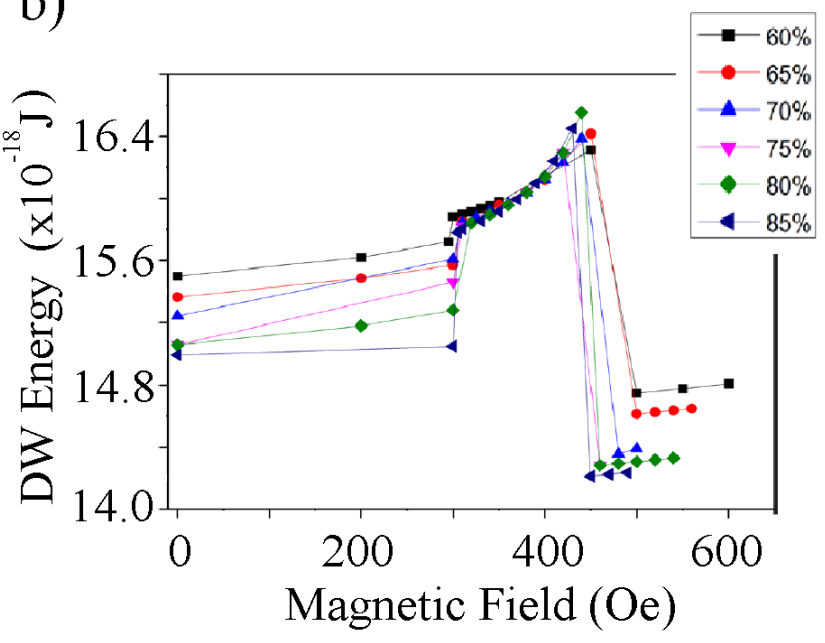

FIG. 32: a) Cross sections of the mixed vortex-transverse domain wall structure moving along the NW. Top image shows a cross-section on the x-z plane and bottom images show a spatial fixed cross-section frames of the x-y plane, taken every $0.1 \mathrm{~ns}$. b) Energy of the domain walls for paramagnetic constraints with different percentages of $\mathrm{Ni}$.

\section{Nucleating an initial transverse DW}

Still in the paramagnetic constraint, we now explore the depinning process when the nucleated domain wall is transversal. The propagation field $H_{\text {prop }}$, is slightly higher for a transversal DW (table III), although the pinning at the chemical paramagnetic defect still happens in a narrow range of external magnetic fields (400-500 Oe) (table III). During the propagation of the transversal DW, in both sections of the NW, its structure is in a mixed vortex-transverse state, although it is purely transversal while pinned. Therefore, the depinning field associated to a $\mathrm{NiCu}$ paramagnetic defect is almost independent of the structure of the nucleated DW because in either case, the structure evolves to a transversal DW while pinned at the defect. 


\begin{tabular}{|c|cc|ccc|}
\hline$N i$ & $H_{\text {prop }} H_{\text {dep }}$ & \multicolumn{4}{|c|}{ Domain wall type at } \\
$(\%)$ & $(O e)$ & $($ Oe $)$ & $1^{\text {st }}$ Part & Spacer & $2^{\text {nd }}$ Part \\
\hline 80 & 310 & 440 & $\mathrm{~V}$ & $\mathrm{~T}$ & $\mathrm{~V}-\mathrm{T}$ \\
75 & 300 & 450 & $\mathrm{~V}$ & $\mathrm{~T}$ & $\mathrm{~V}-\mathrm{T}$ \\
70 & 310 & 470 & $\mathrm{~V}$ & $\mathrm{~T}$ & $\mathrm{~V}-\mathrm{T}$ \\
65 & 300 & 470 & $\mathrm{~V}$ & $\mathrm{~T}$ & $\mathrm{~V}-\mathrm{T}$ \\
60 & 300 & 500 & $\mathrm{~V}$ & $\mathrm{~T}$ & $\mathrm{~V}-\mathrm{T}$ \\
\hline 75 & 440 & 470 & $\mathrm{~V}-\mathrm{T}$ & $\mathrm{T}$ & $\mathrm{V}-\mathrm{T}$ \\
70 & 440 & 490 & $\mathrm{~V}-\mathrm{T}$ & $\mathrm{T}$ & $\mathrm{V}-\mathrm{T}$ \\
65 & 450 & 500 & $\mathrm{~V}-\mathrm{T}$ & $\mathrm{T}$ & $\mathrm{V}-\mathrm{T}$ \\
60 & 440 & 500 & $\mathrm{~V}-\mathrm{T}$ & $\mathrm{T}$ & $\mathrm{V}-\mathrm{T}$ \\
\hline
\end{tabular}

TABLE III: Results obtained for a paramagnetic $\mathrm{NiCu}$ constraint. $H_{\text {prop }}$ and $H_{d e p}$ are the propagation and depinning fields, respectively. The upper half of the table corresponds to a nucleated vortex (V) domain wall (DW). The lower half corresponds to a nucleated transverse (T) DW. Note that the T-DW always propagates exhibiting a mixed vortex-transversal (V-T) structure. .

\section{Ferromagnetic Constraint}

Finally, we have studied the pinning capabilities of a $5 \mathrm{~nm}$ thick $\mathrm{NiCu}$ ferromagnetic constraint. The Curie temperature of $\mathrm{NiCu}$ for small $\mathrm{Ni}$ content is below room temperature (table 1), although a $5 \mathrm{~nm}$-thick constraint may remain ferromagnetic due to the exchange with the adjacent Ni despite its low Curie temperature. Therefore it is worth exploring the pinning capabilities for a ferromagnetic chemical constraint. For an injected vortex DW, the propagation field, $H_{\text {prop }}=300 \mathrm{Oe}$, is quite close to the values obtained in the previous cases. However, due to the magnetic nature of the $5 \mathrm{~nm}$-thick spacer, for $\mathrm{Ni}$ content higher than $55 \%$, the DW does not get pinned at the chemical constraint and it moves unimpeded as a vortex-like until it reaches the opposite end of the NW. The pinning is only achieved for defects with a composition of $\mathrm{Ni} \leq 52 \%$. In those cases, the depinning field is around 900 Oe, considerably higher than the one obtained previously for a non-magnetic spacer. The reasons behind such a high depinning filed can be related to the DW energy. Figure 5 plots its energy during the depinning process. The energy increases abruptly as the DW 
arrives to the chemical constraint due to the change in its shape. Then the energy increases slightly as the structure of the DW is slightly altered under the increasing magnetic field. Finally, the depinning occurs when the field reaches $H_{d e p}$ and the energy drops abruptly to complete the switching.

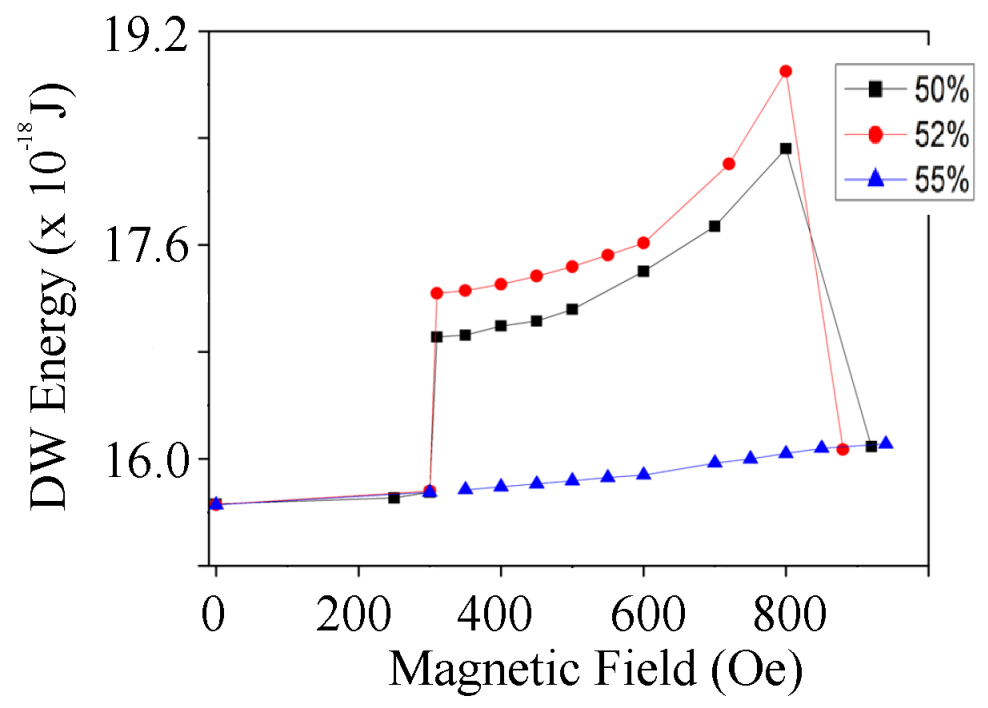

FIG. 33: Plot of the domain wall energy as a function of the external magnetic field applied in the ferromagnetic constraint case. Different curves represent simulations with different percentages of Ni.

\section{E. Consclusions}

To summaraize the results, for constraints much smaller than the width of the DW, the depinning field increases with the width of the defect. For a ferromagnetic constraint, there is no pinning unless the saturation magnetization and the exchange energy of the constraint is reduced notably (for $\mathrm{Ni}$ content smaller than 55\%). However, in this case, when the domain wall gets pinned, the depinning field results quite large (about $900 \mathrm{Oe}$ ). If the constraint behaves paramagnetically, the defect can pin the DW for a much larger saturation magnetization (i.e. Ni content), due to the null contribution of the exchange energy to the energy of the pinned DW. The depinning process in the paramagnetic case is quite similar to the one for a non-magnetic constraint with zero Ni content. The type of the DW injected (vortex or transversal) does not alter the results as the pinned DW adopts always a transversal geometry. 


\section{STRUCTURAL CHARACTERIZATION}

The objective of this thesis is the control of the magnetization dynamics in nanostripes through the application of elastic strain. In order to generate this type of perturbation we use a piezoelectric material (ScAlN), capable to convert electrical signals into strain and viceversa by exploiting the piezoelectric effect. The first part of this chapter is oriented to the structural characterization of ScAlN thin films, whose piezoelectric properties depend on its structural characteristics. X-ray diffraction is performed on thin films of ScAlN samples used as substrate for the fabrication of our devices. Furthermore, piezolectric force microscopy (PFM), Rutherford backscattering (RBS) and measurements of the $d_{33}$ constant are performed on identical ScAlN samples, exclusively fabricated for their characterization.

The goal of the second part of this chapter is to describe the structural characteristics of $\mathrm{Ni}$ thin films that are grown along the magnetic nanostripes that are the core of our study. As both nanostructures are grown simultaneously and nearby each other by sputtering deposition, we assume that their crystalline properties are identical. The magnetic behaviour of the nanostripes is dependent on their crystalline properties, therefore, it is useful to perform X-ray studies in order to achieve a better understanding of the magnetic behaviour of the nanostripes. We perform X-ray diffraction measurements on the Ni thin films. Outof-plane $2 \theta$ scans are carried out in order to obtain the diffraction peaks of the crystals whose Bragg planes are parallel to the surface of the sample. A deeper picture is achieved by analyzing the FWHM of the diffraction peaks in order to compute the mean crystallite size through the Scherrer equation. Grazing incident diffraction (GID) scans are performed to detect signal from randomly oriented grains. This measurement complements the out-ofplane $2 \theta$ scans. Finally, rocking curves are carried out in order to study the dispersion and orientation of the crystals with respect to the sample surface. 
In polycrystalline piezoelectric thin films, the piezoelectric response depends on the microstructural properties of the film, such as grain size, crystallographic dispersion and polarity. Since the introduction of AlN films as piezoelectric materials, it has been used in many applications such as surface acoustic waves (SAW) and bulk acoustic waves (BAW), due to its high acoustic velocity $(6000 \mathrm{~m} / \mathrm{s})$ and high piezoelectric coefficients $d_{31}(2.65 \mathrm{pm} / \mathrm{V})$ and $d_{33}(5.53 \mathrm{pm} / \mathrm{V})$.

Many studies stress that the piezoelectric response of AlN films depends strongly on the crystal orientation. The crystal orientation distribution is measured by the X-ray diffraction method of the rocking curve, which measures the angular dispersion of the grains with respect to the surface normal. These studies base their results in the FWHM of the rocking curves around the peak of (0001) of the AlN. They conclude that the piezoelectric response is enhanced by the decreasing of the FWHM of the rocking curves [F. Martin,2004]. On the other hand, there are also studies which seem to contradict these results by obtaining poor piezoelectric response as the FWHM of the rocking curves is decreased [Sanz-Hervas, 2007].

(a)

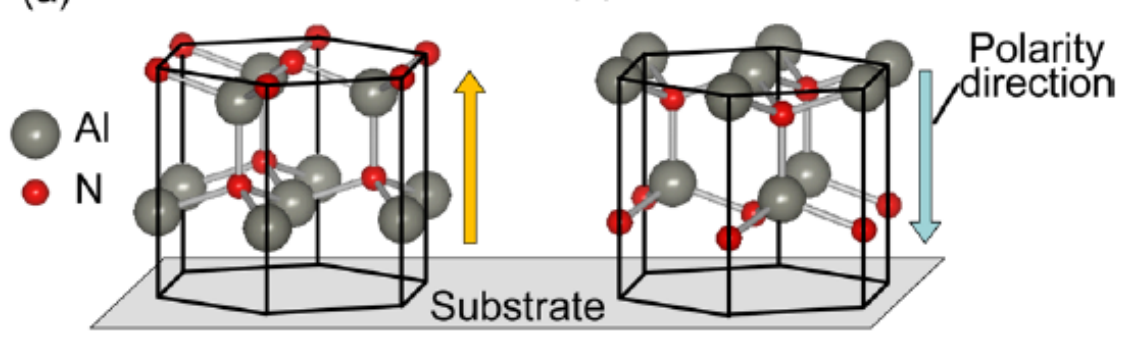

FIG. 34: AlN Wurtzite structure. The c-axis oriented AlN film can be classified as either an (000-1) N-polarity shown (a) or an (0001) Al-polarity shown in (b).

The main characteristic that correlates with a good piezoelectric response is the polarity of the piezoelectric film. The polarity is a vector field that expresses the direction towards which the dipoles of the compound point respect to the surface normal of the sample. Figure 34 show the Wurtzite structure of the AlN compound and its two different polarities according to their definition. In their study, [kamohara et al, 2008] proved that polarity distribution is a significant factor for the piezoelectric response of oriented AlN films, as shown in figure 35. 


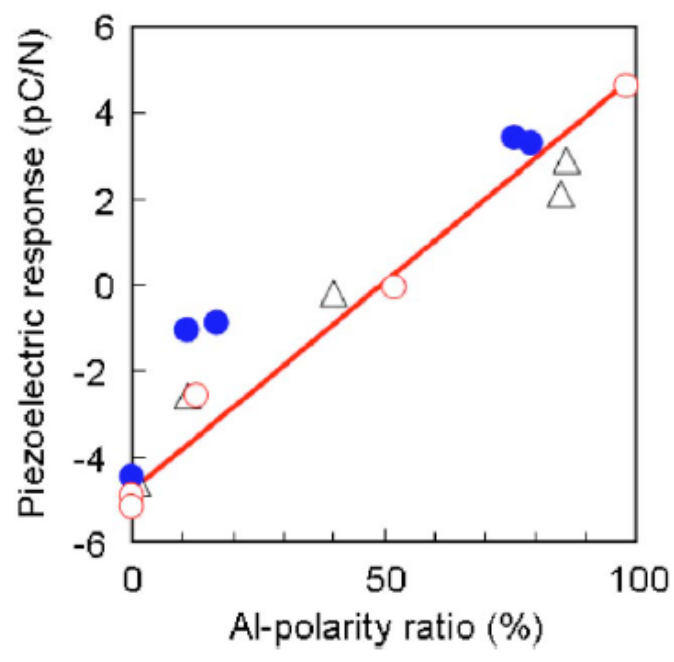

FIG. 35: Piezoelectric response as a function of polarity distribution in oriented AlN films. The sign changes from negative to positive means that the predominant polarity of the AlN films inverts from $\mathrm{N}$ polarity to $\mathrm{Al}$ polarity. Polarity is measured by piezoresponse force microscopy (PFM) [Kamohara et al, 2008]

In Figure 35 the positive sign of piezoelectric response means that the spontaneous polarization of the AlN films is predominantly oriented pointing from the film surface toward the substrate interface(Al polarity). The negative sign means the opposite (N polarity). Following studies have been carried out in order to control the polarity of the AlN films with the goal of controlling its piezoelectric response. For purposes of good piezoelectric response the capability of controlling the polarity is crucial. [Kamohara et al, 2008] proved the crystal orientation and polarity of AlN films grown by magnetron sputtering, is strongly correlated with the sputtering power. The piezoelectric response of AlN films gradually increases from negative to positive sign with increasing sputtering power (Figure 36). This is because there is a dependence between the sputtering power and the FWHM, (the FWHM decreses as the power increases) but also because there is a dependence between the polarity of the film and the sputtering power.

[Milyutin, et al 2010] demonstrated that the polarity can be controlled as well, by the seed layer of the sputtered AlN is grown onto. In this work they were able to control the polarity of the AlN by a seed layer of AlN deposited by molecular beam epithaxy (MBE) or metal organic phase vapour epitaxy (MOPVE) techniques. The low electromechanical coupling coefficient $k_{t}(6 \%-7 \%)$ is a problem in (0001) AlN film resonators, even though 


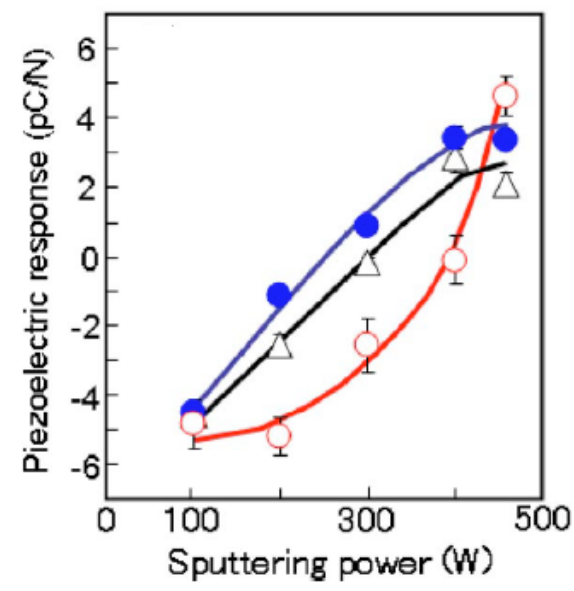

FIG. 36: Dependence of the piezoelectric response of AlN films on sputtering power. Different curves stand for different substrates. [Kamohara et al, 2008]

they possess a high Q factor . Recently, an enhancement of piezoelectricity was found in Sc doped AlN films. The ScAlN film has attracted attention as a potential film bulk acoustic wave resonator (FBAR) material because it has both a high electromechanical coupling value $\left(k_{t}\right)$ and a high Q factor [Winqvist, 2010].

In our work we have utilized as a piezoelectric the ScAlN. The AlN structure stated early is the same for the ScAlN, where the atom of Sc relplace the atom of Al, therefore its composition is $S c_{1-x} A l_{x} N$

\section{A. Characterization of ScAlN Thin Films}

We perform a characterization of the piezoelectric ScAlN thin film before any further nanofabrication process in order to assure its crystal distribution as well as its piezoelectric response. All of our samples are grown on a $\mathrm{Si} / \mathrm{SiO}_{2}$ substrates. The ScAlN is grown on top of it with a thickness of $2 \mu \mathrm{m}$ by reactive sputtering. The ScAl target is kept under a RF power square signal with amplitude of $500 \mathrm{~W}$ and period of $T=161 \mu s$, at a Nitrogen pressure of 3 mtorr. 
Test Samples are grown in order to measure its piezoelectric behaviour. As they needed further procedure, test samples were not suitable for nanodevice fabrication. Measurements on these samples are carried out in the "Groupe de recherche en physique et technologie des couches minces (GCM)", University of Montreal and in the "Emerging materials department, piezoelectric group" (EMDP) at Fraunhofer Institute for Applied Solid State Physics (IAFF)

Rutherford Backscattering spectrometry (RBS) is performed to determine the composition of the ScAlN films. The RBS measurements and analyses of the ScAlN thin films synthesized with the alloy target have been carried out in the GCM, University of Montreal by Martin Chicoine. The results by this technique show that under typical growing conditions the composition is $S c_{0.43} A l_{0.57} N$. Piezoresponse Force Microscopy (PFM) is performed on the test samples. The PFM measurements and analyses have been carried out using a Veeco multimode atomic force microscope (AFM) with a Nanoscope IV controller, by the EMDP group at Fraunhofer Institute for Applied Solid State Physics (IAFF). PFM is done in contact mode by using $5 \mathrm{~V}$, at $650 \mathrm{kHz}$ (contact resonance) frequency, with NSG01-Pt tips for the piezoelectric phase mapping. Periodically poled lithium niobate (PPLN) calibration sample was first measured to ensure $180^{\circ}$ phase difference between opposite polarities in the material. Results, shown in figure 37 (b), show a homogeneous N polarity.

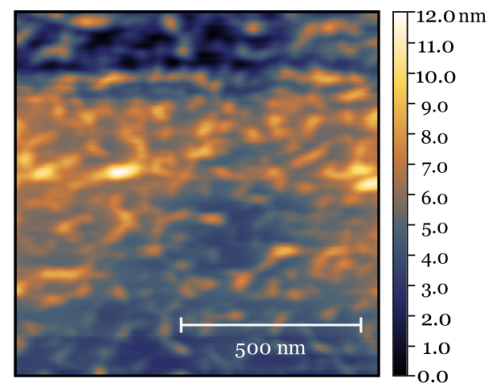

Topography

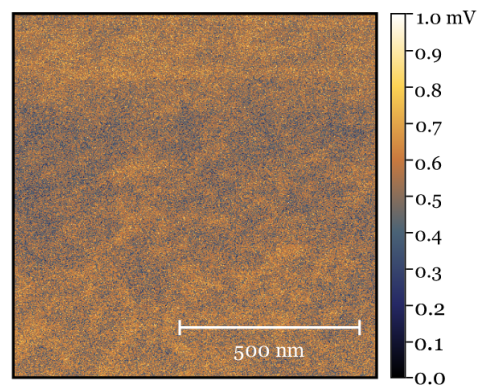

Piezoelectric amplitude

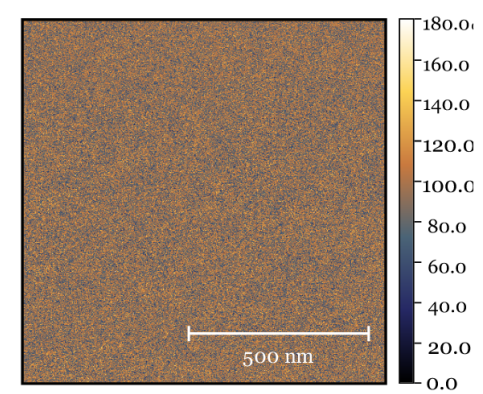

Piezoelectric phase

FIG. 37: a) AFM image of the surface of ScAlN film, b) PFM amplitude of the surface of the ScAlN. Bright contrast indicates N polarity, c) PFM phase

Measurements of the clamped piezoelectric effective constant $d_{33}$ were performed in a Berlincourt piezometer (PM300from Piezotest). The piezoelectric constant $d_{33}$ measure- 
ments were carried out using a Berlincourt piezometer (PM300 Piezotest), by the "Emerging materials department, piezoelectric group" at Fraunhofer Institute for Applied Solid State Physics (IAFF). For contact stability between the sample and the probe, titanium top electrodes were sputtered on top of ScAlN through a shadow mask. The samples were clamped with a constant force of $10 \mathrm{~N}$. An oscillating load of $0.25 \mathrm{~N}$ at a frequency of $110 \mathrm{~Hz}$ was applied and the stress-induced charge was measured and compared to a reference sample. No correction of the experimental $d_{33}$ values for clamping effects was performed. Results for piezoelectric response by this method show a piezoelectric constant whose value is $d_{33}=-24$ $p C / N$

Samples for nanofabrication All piezoelectric films are grown alongside a calibration $10 \times 10 \mathrm{~mm}$ sample with a shadow mask, to form a step on it. That step is later measured in profilometer to assure the thickness grown of ScAlN on the substrate is accurate. After deposition, samples are taken to the X-ray diffractometer to perform out-of-plane $2 \theta / \theta$ scans. In a typical scan we can observe four peaks: at $2 \theta=32.83^{\circ}$ at $2 \theta=35.58^{\circ}$, at $2 \theta=69.13^{\circ}$ and at $2 \theta=75.5^{\circ}$. The first diffraction peak corresponds to the $\operatorname{ScAlN}(0,0,0,1)$ reflection, the second peak corresponds to the $\operatorname{ScAlN}(0,0,0,2)$ reflection, the third one is the $\operatorname{Si}(4,0,0)$ reflection and the last one to the ScAlN $(0,0,0,4)$ [Martin et al, 2004]. This is consistent with previous studies. The hexagonal axis (c axis, [0001]) is the polar direction, and at suitable process conditions, ScAlN grows preferentially with c-axis orientation on SiO2, [Englemark, 2001]. In order to measure the crystal dispersion respect to the sample surface normal of the ScAlN, we perform a rocking curve around one of the peaks. We choose the most intense peak, therefore the rocking curve measurement is performed around the $(0,0,0,2)$ reflection (figure 38).

As stated before, a narrow FWHM of the rocking curves by itself is not enough condition to assure the ScAlN to have a piezoelectric response. However, previous measurements of $d_{33}$ coefficient and PFM measruements of test samples films grown in the same sputtering conditions plus these rocking curves, will be sufficient to suppose the piezelectric beahaviour. The range of FWHM measured of all the ScAlN films were $\left[1.7^{\circ}<\mathrm{FWHM}<7.8^{\circ}\right]$. No sample showed a FHWM larger than $7.8^{\circ}$, and all samples gave evidence of piezoelectric response after the devices nanofabrication, at calibrating the SAW resonators in the Network 

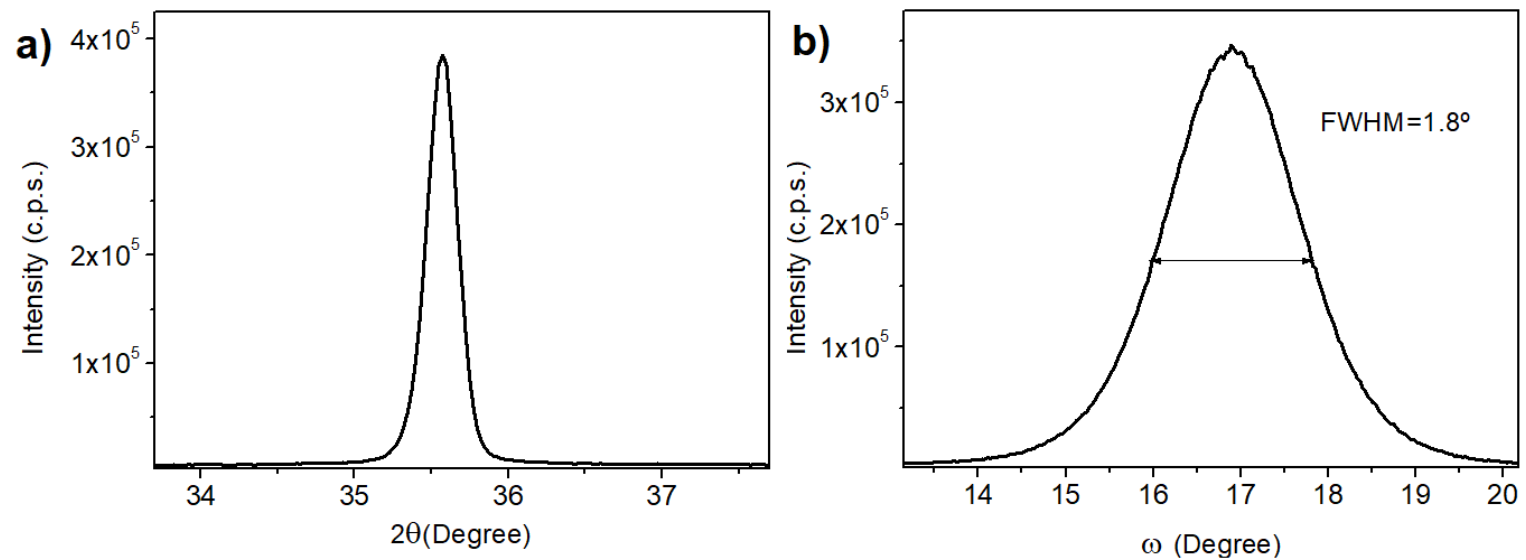

FIG. 38: X-ray diffraction measurements of $2 \mu \mathrm{m}$ thin film of ScAlN grown on top of $\mathrm{SiO}_{2}$, a) is a $\theta / 2 \theta$ scan centered in the $(0,0,0,2)$ reflection and $\mathrm{b})$ is the rocking curve around that reflection

Analyzer.

\section{B. Characterization of Ni Thin Films}

As the core of our devices in this work are the magnetostrictive nanostripes, we perform some structural study of the spontaneous crystallization of Ni. Such X-ray characterization are carried out on $35 \mathrm{~nm}$-thick films of $\mathrm{Ni}$ deposited by DC magnetron sputtering. This section summarizes the results of the structural analysis. The subsections presented here, cover the out-of-plane $\theta / 2 \theta$ measurements where we find which planes are the Ni preferentially grown in the out-of-plane direction, and we also estimate the grain size of the $\mathrm{Ni}$ in the out-of-plane dimension by the Scherrer equation. Grazing incident scans are performed as well in order to determine the possible planes that grow away from the surface normal. Finally we also determine the mosaicity of the samples by performing rocking curves. The Ni nanowires and nanostripes in the literature are known by their polycrystalline structure [Kozlov, 2019] [Rheem,2007] [Pan, 2005] as well as the difficulties in the magnetic measurements that this implies. This reason justify the crystallographic study of the sputtering deposited Ni. 
out-of-plane $\theta / 2 \theta$ scan Samples with structure Substrate/ScAlN $(2 \mu m) / \operatorname{Cr}(4$ $\mathrm{nm}) / \mathrm{Ni}(35 \mathrm{~nm}) / \mathrm{Pt}(2 \mathrm{~nm})$ were deposited alongside the deposition of the nanostripes fabricated via lithography by magnetron sputtering. A Cr layer is deposited as a buffer in order to improve the adhesion of the Ni to the ScAlN piezoelectric. A Ta layer was also tried as a buffer with poor adhesion results for the nanostripes. Samples were taken afterwards to the X-ray diffractometer to perform out-of-plane $\theta / 2 \theta$ scans. The XRD out-of-plane patterns are presented in figure 39. It coincides in all its features with the previous $2 \mu m$ film of ScAlN, plus a peak at $44.6^{\circ}$ which corresponds to the $\mathrm{Ni}(111)$ diffraction peak. Using the Bragg's law, the interplanar spacing of the Ni can be calculated from $2 \theta_{(111)}=44.58^{\circ}, d=2.031 \AA$. Hence the lattice constant $a$ can be derived using the formula for cubic crystals:

$$
\frac{1}{d^{2}}=\frac{h^{2}+k^{2}+l^{2}}{a^{2}}
$$

where $h, k$ and $l$ are the Miller indices of the plane considered. This leads to $a_{N i}=3.516$ $\AA$.

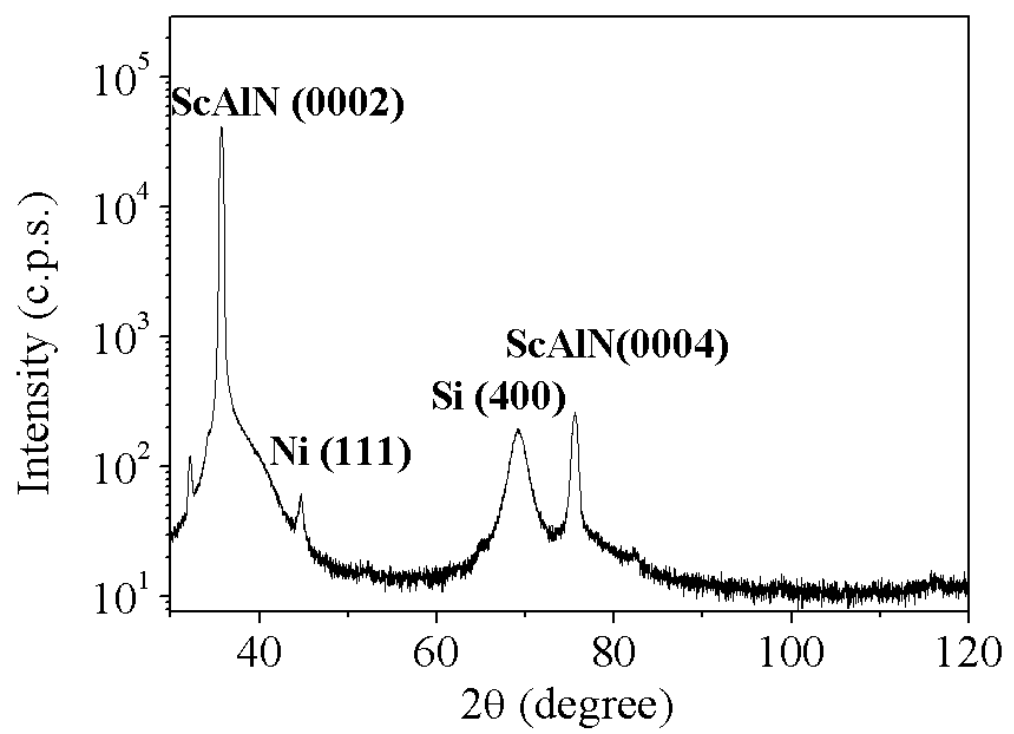

FIG. 39: $2 \theta / \theta$ measurements of $35 \mathrm{~nm}$ Ni film deposited over the piezoelectric $2 \mu m \mathrm{ScAlN}$

Once the $2 \theta / \theta$ scan is performed, we focus on the only peak in this pattern that has the Ni. Considering that the out-of-plane $2 \theta / \theta$ is sensitive only to crystalline planes parallel to the sample surface, we can conclude that $\mathrm{Ni}$ (111) planes grow textured in the normal to the surface direction. 
Scherrer equation The analysis of crystallization process can be improved by using the Scherrer equation, a formula that allows to estimate the mean crystallites dimension, also known as grain size. In a polycrystalline metal or alloy the grain size has pronounced effects on many of its properties. In our case for example, the grain size of Ni crystallites has effect in the coercive field of the studied nanostripes [Loeffel, 1998].

The Scherrer equation is the result of mathematical considerations about the finite periodicity of equally spaced planes and it relates the size of sub-micrometer crystals to the broadening of diffraction peaks in XRD measurements. The Scherrer equation can be employed to estimate the average grain dimension as

$$
t=\frac{K \lambda}{B \cdot \cos \theta_{B}}
$$

where $t$ is the mean crystallite size, $K$ is a shape factor whose value is close to unity, $\lambda$ is the wavelength of the radiation employed, $\mathrm{B}$ is the full width at half maximum (FWHM) of the peak and $\theta_{B}$ is the incident angle which satisfies the Bragg condition. The Scherrer equation gives an estimation of the crystallites dimension along the direction perpendicular to the Bragg plane. Therefore, $2 \theta / \theta$ scan will estimate the grain size along the depth of the film. This is evidently limited by the thickness of the layer.
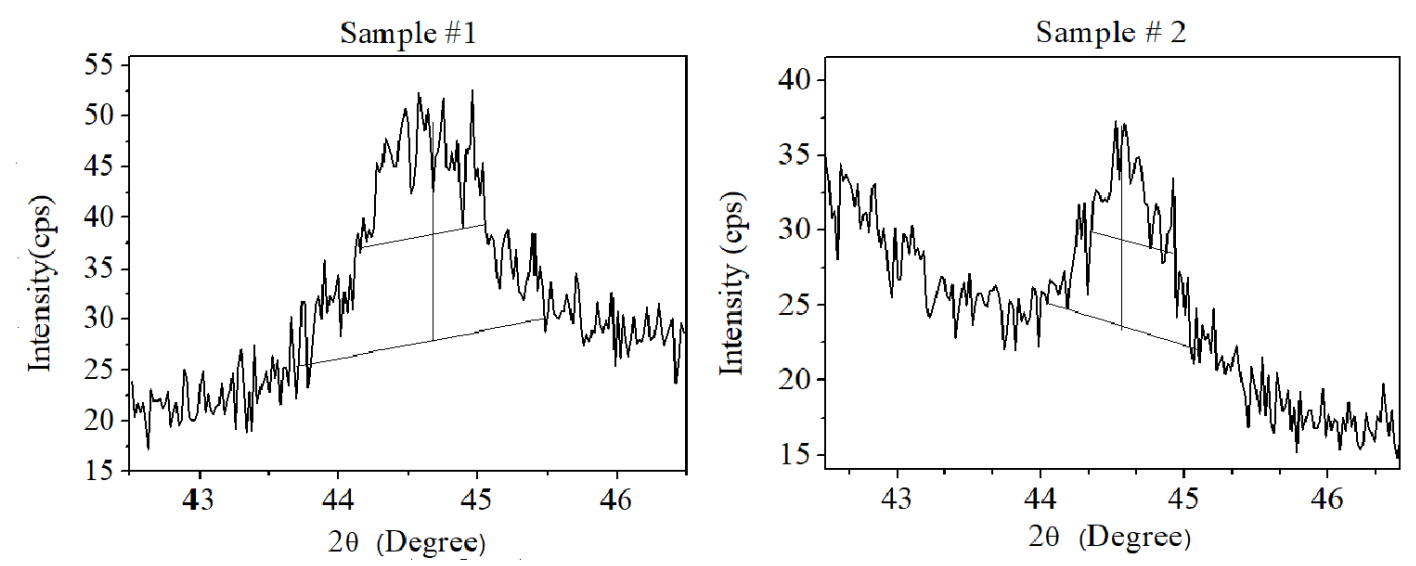

FIG. 40: Peaks of the $\mathrm{Ni}(111)$ in $2 \theta / \theta$ scans. Figure a) shows the peak of the scan of the first set of samples and Figure b) shows the peak for the second set of samples.

We have worked in this thesis with two sets of Ni samples. They are grown out of different targets of $\mathrm{Ni}$ and they are both analyzed. We measured the FWHM of the Ni(111) peak 
in the $2 \theta / \theta$ scan for each sample and estimate the grain size dimension along the normal direction to the surface sample.

$$
\begin{aligned}
& \text { For sample \# } 1 B_{1}=0.92^{\circ} \\
& \text { For sample \# } 2 B_{2}=0.45^{\circ}
\end{aligned}
$$

Having into account that the X-rays wavelength $\lambda=1.451 \AA$ and $\theta_{B}=22.3$. The grain size for each sample is

$$
\begin{aligned}
& t_{1}=11 \mathrm{~nm} \\
& t_{2}=18 \mathrm{~nm}
\end{aligned}
$$

In order to estimate the grain size in all dimensions, we need to perform In-plane $\phi / 2 \theta_{\chi}$ scans. In these measurements the Bragg condition is fixed always perpendicular to the sample surface. So this type of scans complements the $2 \theta / \theta$ scans to the grain size estimation. The PANalytical X'pert Pro diffractometer of ISOM-UPM has not the possibility of performing these kind of scans. However, we have no reason to think that the grains has different sizes in the in-plane dimensions, especially when the grain size in the perpendicular direction is smaller than the thickness of the Ni layer. If the grain size were the same dimension as the film thickness we could have argued that the grain size in the in-plane directions could have been larger. Therefore we conclude these as the sizes of the Ni(111) crystallites.

Grazing Incident Diffraction (GID) scans are suitable for studying non-textured polycrystalline thin films, since at low incident angles the large irradiated volum allows to detect the diffracted signal from randomly disordered grains [Guinebretiere, 2007]. Therefore, out of plane $2 \theta / \theta$ and GID measurments give complemetnary information about the crystalline structure of sample. Figure 41 shows the GID diffractogram of our sample. The complete structure is Substrate/ScAlN $(2 \mu m) / \mathrm{Cr}(4 \mathrm{~nm}) / \mathrm{Ni}(35 \mathrm{~nm}) / \mathrm{Pt}(2 \mathrm{~nm})$. The GID scan presented here was taken with an incident angle $\alpha=0.5^{\circ}$. We can identify several peaks, which not only match with the $\mathrm{Ni}$ peaks, but also with the $\mathrm{Cr}$, Pt and even the ScAlN one. $\mathrm{Ni}(111)$ peak may be found at $2 \theta=44.58^{\circ}$, which is not certain as the peak of $\mathrm{Cr}(111)$ is at a very similar angle reflection. We also find $\mathrm{Ni}(200)$ at $2 \theta=52.01^{\circ}, \mathrm{Ni}(220)$ at $2 \theta=76.46^{\circ}$ and $\mathrm{Ni}(311)$ at $2 \theta=93.07^{\circ}$. 


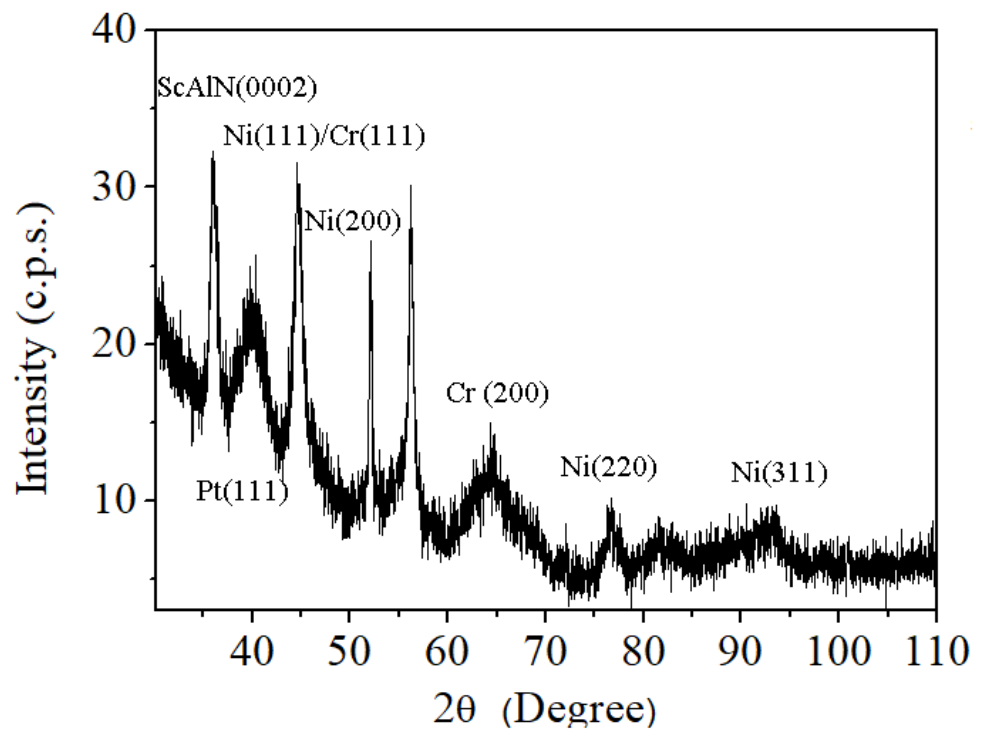

FIG. 41: GID diffractogram of a sample whose structure is Substrate $/ \operatorname{ScAlN}(2 \mu m) / \mathrm{Cr}(4 \mathrm{~nm}) / \mathrm{Ni}(35 \mathrm{~nm}) / \operatorname{Pt}(2 \mathrm{~nm})$. Different peaks from different materials are identified.

The fact that we can identify more peaks of $\mathrm{Ni}$, tells us that $\mathrm{Ni}$ grows polycrystalline, preferentially textured in the $\mathrm{Ni}(111)$, but also crystals with other planes grow in a nontextured way. Peaks from more materials of our sample structure are found. Cr, which is used as a buffer layer is found, and its first peak may be overlapping the $\mathrm{Ni}(111)$ peak at $2 \theta=44.4^{\circ}$. The peak at $2 \theta=64.4^{\circ}$ corresponds to the $\operatorname{Cr}(200)$. We also observe a peak corresponding to $\mathrm{Pt}$, which is used as a capping layer, $\mathrm{Pt}(111)$ is found at $2 \theta=39.8^{\circ}$.

Rocking curves We make a study of the mosaicity of the $\mathrm{Ni}(111)$, by performing rocking curve measurments. Mosaic structure is a kind of substructure where the crystal is divided in tiny blocks which are distributed along the surface in an enormously exaggerated fashion. These blocks are what we call grains. Each grain is disoriented from one another and misaligned with respect to the sample surface. [Cullity, 2014].

A rocking curve consists in measuring the intensity of a given diffraction peak, at a fixed Bragg condition as a function of rotation $\omega$ of the sample. The width of a rocking curve is a direct measure of the range of orientation present in the irradiated area of the crystal, 


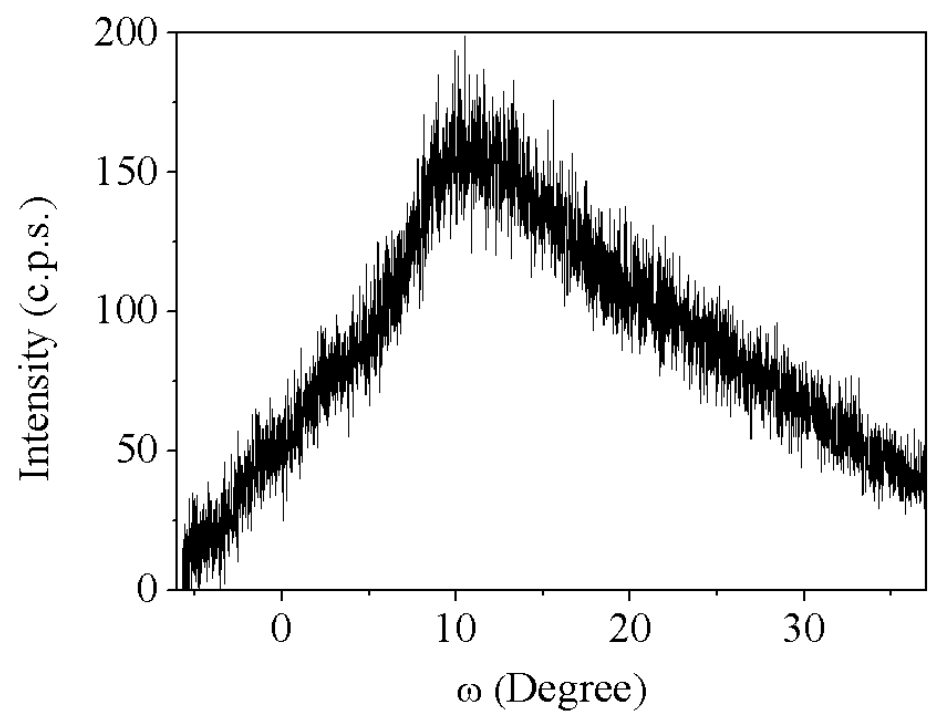

FIG. 42: XRD $\omega$ scans (rocking curves) of the Ni(111) reflection.

because each grain of a typical mosaic crystal successively comes into reflecting position as the crystal is rotated. This way, the scan estimates the out-of plane texture dispersion of the grains. The FWHM of a rocking curve indicates the average misalignment of textured grains with the sample surface. Figure 42 shows the rocking curve of the Ni(111) reflection. The rocking curve is not centered in zero, which means there is not good alignment of the $\mathrm{Ni}(111)$ lattice planes with the sample surface. This implies that the preferential orientation of the $\mathrm{Ni}(111)$ is tilted about $10^{\circ}$ with respect to the direction normal to the surface sample. The FWHM is about $15.05^{\circ}$, which indicates a relatively large texture dispersion of the $\mathrm{Ni}(111)$ crystals.

\section{Conclusions}

This chapter was oriented to the characterization of the ScAlN and the Ni thin films. The structural characterization of the ScAlN thin films was necessary in order to assure its piezoelectric behaviour. A first set of measurements were performed in test samples not suitable for device fabrication. These test samples were grown in an identical fashion as the ScAlN thin films used for the device fabrication. Test samples were sent to the Groupe de recherche en physique et technologie des couches minces, at the University of Montreal and Rutherford Backscattering spectrometry was performed on them in order to determine 
the composition. RBS measurements show that under typical growing conditions the composition is $S c_{0.43} A l_{0.57} N$. Piezoresponse Force Microscopy (PFM) was also performed in this samples in order to check that the polarity of the ScAlN thin films. Result show homogeneous $\mathrm{N}$ polarity in the test samples. The polarity is found in the literature as the main characteristic that relates to the piezoelectric behaviour of the thin films. Finally, measurements of the $d_{33}$ parameter were performed in the test samples. Results for piezoelectric response show a piezoelectric constant whose value is $d_{33}=-24 p C / N$. These measurements were carried out in order to verify the piezelectricity behaviour of the samples grown by our sputtering method. Samples of ScAlN thin films that were used for the fabrication of devices underwent X-ray diffraction measurements, where we could find the peaks corresponding to the $\operatorname{ScAlN}(0,0,0,1),(0,0,0,2)$ and $(0,0,0,4)$. In order to measure the crystal dispersion respect to the sample surface normal we perform rocking curves around the $(0,0,0,2)$ reflection. Rocking curves measurements for this reflection show a full width at half maximum in a range of $\left[1.7^{\circ}<\mathrm{FWHM}<7.8^{\circ}\right]$.

$\mathrm{Ni}$ thin films that are grown alongside the Ni nanostripes in the sputtering chamber, underwent X-ray diffraction measurements. Out of plane $2 \theta / \theta$ measurements show that Ni grow textured in the plane $(1,1,1)$. The Scherrer equation is used to perform a deeper analysis of the $2 \theta / \theta$ scans. By measuring the full width at half maximum of the $\mathrm{Ni}(1,1,1)$ peak, we are able to estimate the mean size of the crystallites in the direction perpendicular to the film plane. Throughout this thesis we have worked with Ni samples grown out of two different Ni targets. Ni thin films show two different grain sizes, $t_{1}=11 \mathrm{~nm}$ and $t_{2}=18$ $n m$. Grazing incident diffraction measurements are performed in the Ni thin films in order to give complementary information to the $2 \theta / \theta$ scan. Due to the GID diffractogram we can identify that besides the $\mathrm{Ni}(1,1,1)$ planes, the crystals grow with other planes in a nontextured way $(\mathrm{Ni}(2,0,0)$ and $\mathrm{Ni}(2,2,0))$. Finally we perform a rocking curve of the $\mathrm{Ni}(1,1,1)$ diffraction peak, which is a direct measurement of how the crystals are oriented with respect to the sample surface. Results show a FWHM about $15.05^{\circ}$, which indicates a relatively large texture dispersion of the $\mathrm{Ni}(1,1,1)$ crystals. 


\section{MAGNETIC CHARACTERIZATION}

In the pervious chapter we studied the crystalline properties of $\mathrm{Ni}$ films whose structures are identical to the nanostripes fabricated through this thesis. This chapter is aimed to the study of the magnetic anisotropy of those thin films, by studying the dependence of the magnetization on the orientation of the thin film. The two types of magnetic anisotropies that are relevant for this work are the shape anisotropy and the crystal anisotropy. In this chapter we describe the study of preferential direction of magnetization of the Ni thin films that are fabricated along with the nanostripes, with the same structure and we describe the VSM measurements performed on the thin films along different directions. Out of these measurements easy and hard axis in the film, as well as anisotropy constants can be calculated.

Magnetocrystalline Anisotropy The magnetocrystalline anisotropy occurs in a crystal lattice if it takes more energy to magnetize in certain directions than in others. These directions usually coincide with the principal axes of the crystal lattice. The magnetocrystal anisotropy energy (MAE) arises from the spin-orbit coupling. This comes from the fact that in a real lattice, the orbital magnetic moments are quenched, and its orientation is fixed very strongly. As there is a coupling between the spin and the orbital motion of the electron, orientating the magnetic moments away from the crystalline direction, requires an external source of energy. The energy required to rotate the magnetization away from the easy direction is called the anisotropy energy and it is defined as the energy necessary to overcome the spin orbit coupling.

Uniaxial anisotropy It refers to the existence of one only easy direction. If the easy axis is defined in the $\mathrm{z}$ direction, thus, the anisotropy energy will be rotationally-symmetric with respect to the $\mathrm{z}$ direction and will depend only on the relative orientation of the magnetization with respect to this axis. The uniaxial anisotropy energy can be expressed as :

$$
E=K_{0}+K_{1} \sin ^{2} \theta+K_{2} \sin ^{4} \theta+\ldots
$$


When $K_{1}$ and $K_{2}$ are both positive, the energy $\mathrm{E}$ is minimum for $\theta=0$, and the $\mathrm{z}$ axis is an axis of easy magnetization. A crystal with a single easy axis, along which the magnetization can point either up or down, is referred to as a uniaxial crystal. Its domain structure in the demagnetized state is particularly simple. This is the case for example, of Co, which is known for having an hexagonal structure and whose preferential magnetic axis is the c-axis.

Cubic anisotropy When a crystal has a cubic anisotropy, all three main directions in the cubic lattice are susceptible to be either a hard or an easy axis simultaneously. The normal magnetocrystalline anisotropy for cubic structures has this form:

$$
E_{k}=K_{0}+K_{1}\left(\alpha_{1}^{2} \alpha_{2}^{2}+\alpha_{1}^{2} \alpha_{3}^{2}+\alpha_{2}^{2} \alpha_{3}^{2}\right)+K_{2} \alpha_{1}^{2} \alpha_{2}^{2} \alpha_{3}^{2}+\ldots
$$

Where $K_{i}$ are the anisotropy constants and $\alpha_{i}$ are the director cosines. We have two main cases in this type of anisotropy, when $K_{1}>0$ then there are six equivalent energy minima corresponding to the $x, y$ and $z$ directions, both positive and negative. Conversely when $K_{1}<0$ a more complex situation arises with eight equivalent minima along the directions pointing the vertices of the cube (e.g. the direction (111)) and the coordinate axes become now hard axes. It is well known that MAE causes the (111) axis in fcc Ni to be the preferential magnetization direction [Halilov,1997]

Anisotropy in polycristalline samples Polycrystalline magnetic samples, which are formed by an aggregate of crystals (grains) randomly oriented in space, will average out the anisotropy, because of the anisotropy of individual grains. If, on the other hand, crystals have a preferred orientation, also called crystallographic texture, then the polycrystalline aggregate itself will have an anisotropy dictated by the weighed average of the individual grains. The kind of texture possessed by a body depends on its shape and how it was formed. In our specific case, we have observed the polycrystalline nature of the grown-by-sputtering magnetic nanostripes, with a textured tendency of the $\mathrm{Ni}$ (111) crystals to grow paralel to the sample surface, therefore it makes sense to measure the anisotropy since the oriented grains will average a global anisotropy. 


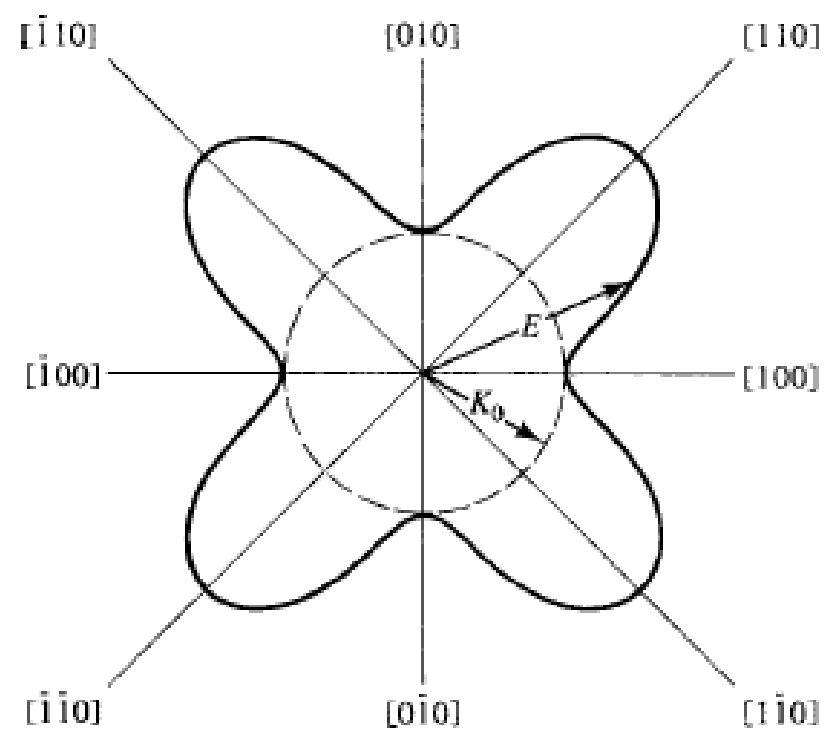

FIG. 43: Polar plot of crystal anisotropy energy as a function of the direction of the planes of a cubic crystal in $z=0$, [Cullity, 2008]. In this plot the easy axis are parallel to (100) and (010) like the case of $\mathrm{Fe}\left(K_{1}>0\right)$. In the $\mathrm{Ni}$ case the preferential axes would be (111) since $K_{1}<0$

Shape Anisotropy The demagnetizing field is the magnetic field $\left(H_{d}\right)$ generated by magnetization within a magnetic sample. The total magnetic field in the magnet is a vector sum of the demagnetizing field and the magnetic field generated by any free or displacement currents. The term demagnetizing field reflects the tendency that it acts on the own magnetization in order to reduce the total magnetostatic energy of the sample. The demagnetizing field in general leads to spatial inhomogeneity of the magnetizaion (e.g. domain formation). This gives rise to the shape anisotropy. When a particle is not perfectly spherical, the demagnetizing field will not be equal for all directions, creating one or more easy axes.

The demagnetizing field and shape anisotropy are usually very complex to compute, but in this case where we study this magnitude in thin films, the computation is easier. For the special case of ellipsoids, which includes objects such as spheres, long thin rods, and thin films, $H_{d}$ is linearly related to $\vec{M}$ by a geometry constant called demagnetizing factor $N$.

$$
\overrightarrow{H_{d}}=-N \cdot \vec{M}
$$


The shape anisotropy creates a favorable energy term for magnetization along the in plane axes and an unfavorable term for magnetization along the surface normal of a (homogeneously magnetized a thin film. For a thin film with magnetization perpendicular to the plane, it approaches unity.

$$
H_{d} \approx-\vec{M}
$$

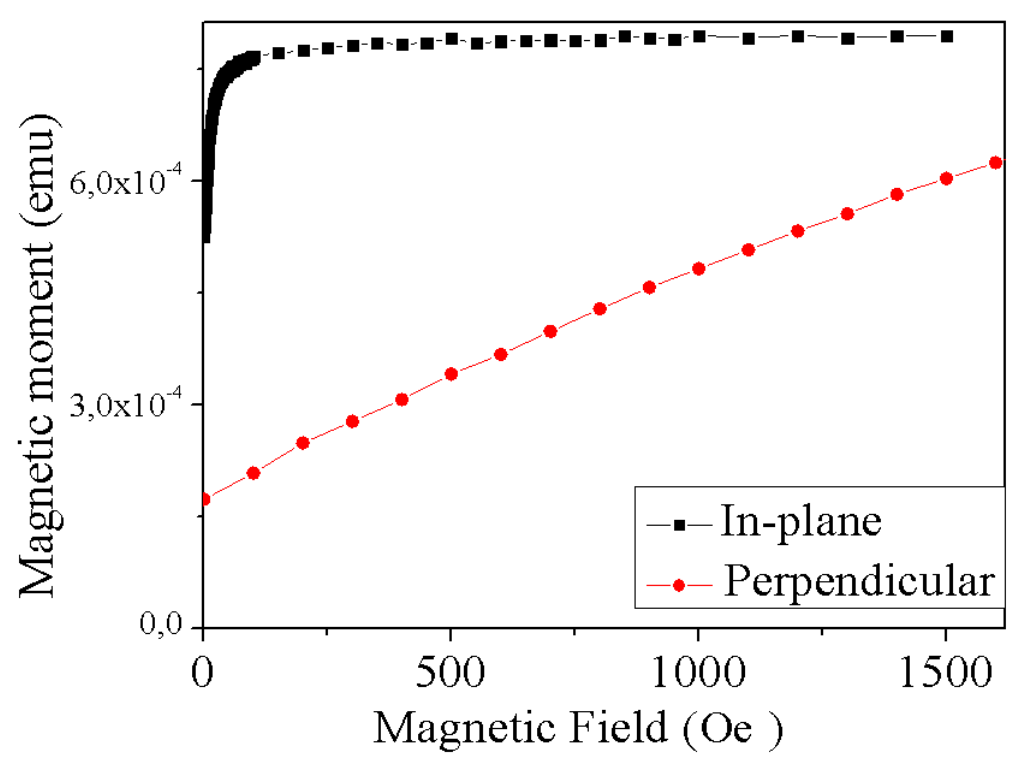

FIG. 44: VSM measurements of a thin film with structure $\operatorname{ScAlN}(2 \mu m) / \mathrm{Cr}(4 \mathrm{~nm}) / \mathrm{Ni}(35 \mathrm{~nm}) / \mathrm{Pt}(2$ $n m$ ). Measurements are performed with the external magnetic field parallel and perpendicular to the surface of the sample.

Figure 44 shows VSM measurements of thin film of $\mathrm{Ni}$ in in-plane and perpendicular directions. We observe the tendency of the film to be magnetized easily in the in-plane direction. This is because of the shape anisotropy.

\section{A. VSM Measurements}

VSM measurements are performed in order to find anisotropy axis as well as anisotropy constants. Dimensions of the substrates where the thin films are deposited are $10 \times 5 \mathrm{~mm}$. Schematics of the in-plane VSM measurements in the Ni thin films is presented in figure 45, 
where $\theta$ is the angle that forms the external magnetic field with the long axis of the sample. Long axis of the magnetic nanostripes is fabricated parallel to the long axis of the sample. For future computations we need the volume of the Ni deposited over the substrate. As the $\mathrm{Ni}$ thin film is $35 \mathrm{~nm}$ thick, the total volume of $\mathrm{Ni}$ is:

$$
V_{N i}=1.75 \cdot 10^{-6} \mathrm{~cm}^{3}
$$

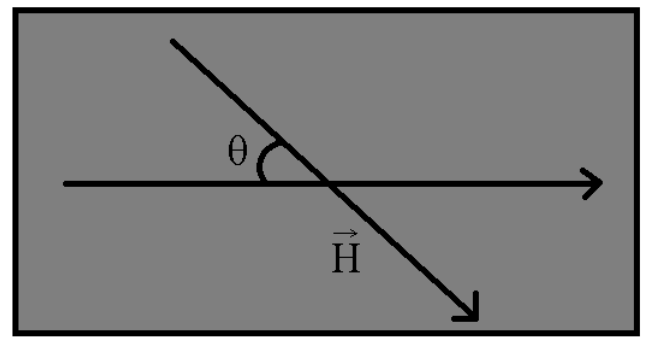

FIG. 45: Schematics of the VSM measurements performed on thin film. $\theta$ is the angle that forms the external magnetic field with the long axis of the sample.

Determining the anisotropy with the area method This method of determining anisotropy constants from magnetization curves is based directly on the definition of the anisotropy energy E, namely, the energy stored in a crystal when it is magnetized to saturation in a non easy direction. If we can calculate W (eq. 17), the work done on the crystal to bring it to saturation, we can equate $\mathrm{E}$ and $\mathrm{W}$ and so compute the anisotropy constants. We determine the energy density by calculating the area that is enclosed between two saturation curves along the two different axes

$$
E=\int_{0}^{M} H d M
$$

As stated in the previous chapter, we have two sets of samples, grown out of different targets of Ni. We perform VSM measurements on these thin films, which are grown simultaneously with the nanostripes of our devices. Our thin films are measured with the same exact structure of the nanostripes, which is Substrate/ScAlN $(2 \mu \mathrm{m}) / \mathrm{Cr}(4 \mathrm{~nm}) / \mathrm{Ni}(35$ $n m) / \operatorname{Pt}(2 n m)$. 
Sample \# 1 The first set of samples with a grain size of $D_{1}=11 \mathrm{~nm}$ is measured. We find the easy axis directions by measuring hysteresis loops in different orientations. In this case the easy axis is found to be forming $45^{\circ}$ with the long axis of the sample and the long axis of the nanostripes, as shown in figure 46. The hard axis is found to be perpendicular to this (still within the plane) at $135^{\circ}$ rotating the thin film $90^{\circ}$ in the plane.

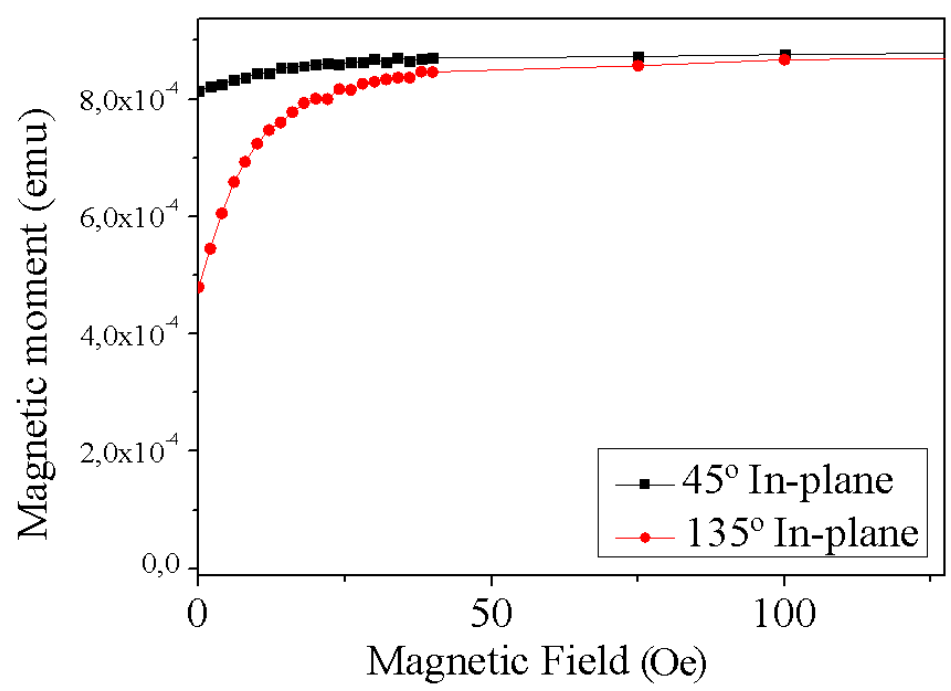

FIG. 46: VSM measurements in the first set of thin films. Black data are taken with an angle of $45^{\circ}$ between the long axis of the sample and the magnetic field, and the red data are taken with an angle of $135^{\circ}$

The area computed out of this first measurement is $0.0048 \mathrm{emu} \cdot G$. In order to obtain the anisotropy energy we have to divide this value between the total volume of the thin film. With proper changes of unities we compute the value of the anisotropy as:

$$
E_{1}=0.3 \cdot 10^{3} \mathrm{~J} / \mathrm{m}^{3}
$$

We treat this anisotropy as an uniaxial anisotropy, which is in fact, an average value of the anisotropy of all the grains (crystals) that form the thin film. As the Ni film grows textured, the Ni (111) planes grow parallel to the sample surface. The value of the anisotropy depends not only on the texture of the sample, but also depends on the crystallites size. 
Sample \# 2 The second set of samples with a grain size of $D_{2}=18 \mathrm{~nm}$ is measured. We find the easy axis directions by measuring hysteresis loops in different orientations. In this case we find the easy axis at $0^{\circ}$ with respect the long axis of the sample and the long axis of the nanostripes. Hard axis is found perpendicular to the long axis at $90^{\circ}$

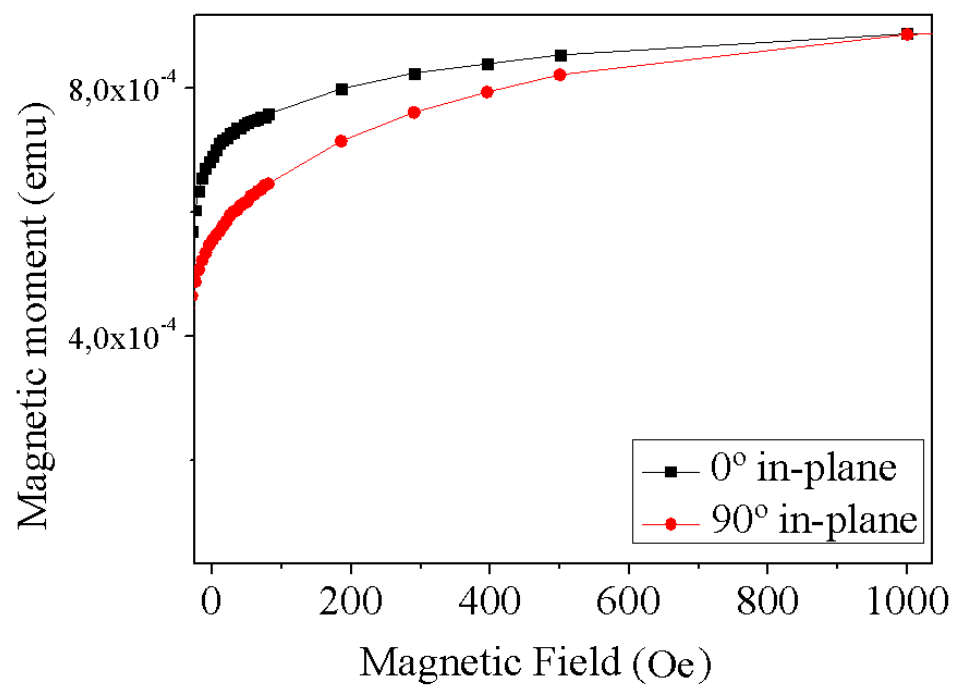

FIG. 47: VSM measurements in the second set of thin films. Black data are taken with an angle of $0^{\circ}$ between the long axis of the sample and the magnetic field, and the red data are taken with an angle of $90^{\circ}$

It is observed in figure 47 that, although there is a preferential axis, there is an anisotropy dispersion, since the approximation to saturation is by rotation, as well in the black curve. The area computed out of this second sample is $0.049 \mathrm{emu} \cdot G$. In order to obtain the anisotropy energy we have to divide this value between the total volume of the thin film. With proper changes of unity we compute the value of the anisotropy as:

$$
E_{2}=2.8 \cdot 10^{3} \mathrm{~J} / \mathrm{m}^{3}
$$

We observe a difference in the anisotropy energy of one order of magnitude. Such a difference in the anisotropy is explained by the different crystal properties, such as the grain size and the dispersion of the Ni crystals. When decreasing the grain size, exchange coupling between neighboring grains may overcome the magnetocrystalline anisotropy of each grain. 
This results in a reduced effective anisotropy $\langle K\rangle$, which represents an average over the magnetocrystalline anisotropies of the correlated grains. For a random orientation of the anisotropy axes of the grains the effective anisotropy is reduced by a factor of $1 / \sqrt{N}$, where $N$ is the number of the magnetically correlated grains. Regarding the crystal dispersion, when crystals have a preferred orientation (crystalographic texture), then the polycrystalline aggregate will have an anisotropy dictated by the weighed average of the individual crystals. Therefore, an enhanced crystal texture turns out as a larger anisotropy.

\section{B. Conclusions}

This chapter was oriented to the measurements of the anisotropy directions and anisotropy constants in $\mathrm{Ni}$ thin films that are assumed to be identical in the crystalline sturcture to the Ni nanostripes which are the core of this thesis. This is carried out by the measurements of hysteresis cycles in a vibration sample magnetometer (VSM) applying the external magnetic field at different orientations. VSM measurements were performed in samples grown out of two different Ni targets by sputtering deposistions, resulting in different characteristics for thin films coming out of different Ni targets. Results show that for the Ni films with smaller grin size the anisotropy energy was noticeably smaller than the thin films whose grain size was larger. This is because when decreasing the grain size, the exchange coupling between neighboring grains may overcome the magnetocrystalline anisotropy of individual grains, resulting in a reduced anistoropy averaged over all the correlated grains. 


\section{STRAINTRONICS DEVICE}

The main straintronics devices fabricated in this thesis are formed by a SAW resonator and a ferromagnetic nanostripe. The SAW resonator is introduced along with the explanation of the generation of surface acoustic waves in the Appendix in page 151. The characterization of the SAW resonator is carried out by the measurement of the $S_{11}$ parameter in a network analyzer. By this, the fundamental excitation modes of the SAW resonator are found. The magnetic dynamics of the nanostripes are studied from anisotropic magnetoresistance measurements (AMR) [Smit, 1951] [Fert, 1968] [Campbell, 1970]. From AMR, the presence of DW's can be directly measured through the resistance of the magnetic layer that carries them, since the AMR effect is a bulk scattering phenomenon. The AMR effect is typically small, a few percents at room temperature compare to GMR [Baibich, 1988], [Pratt, 1991], [Yuasa, 2003]. One particular advantage of a GMR structure, is that the resistance of the nanowire varies almost linearly with the position of the DW along the nanowire so that the position of the DW can be directly determined. This is not the case with AMR which only detects the presence of a DW regardless of its position.

\section{A. Device Description}

The device fabricated is formed by two elements and it is shown in figure 48. The left side half of the device is a surface acoustic wave (SAW) resonator (figure 48 a). The SAW resonator consists in four pads and an interdigital transducer (IDT). The IDT is a periodic array of metallic bars, which are alternatively connected to different pads. When the SAW resonator is at function, the signal is applied to the contact pad number 2, whereas the rest are connected to ground. Contact pads 1, 3 and 4 are shorted between them and half of the metallic bars. The right half of the device includes the ferromagnetic nanostripe and four contact pads in order to carry out four point measurements. The pads and the nanostripe are in contact through two current lines.

A close-up of the IDT is shown in figure $48 \mathrm{~b}$ ). The periodicity is $2.8 \mu \mathrm{m}$ that is the wavelength $(\lambda)$ of the generated SAW's. The nanostripe is placed as close as possible to the IDT $(L=40 \mu m)$, for the SAW's to reach the nanostripe with minimum dissipation. 


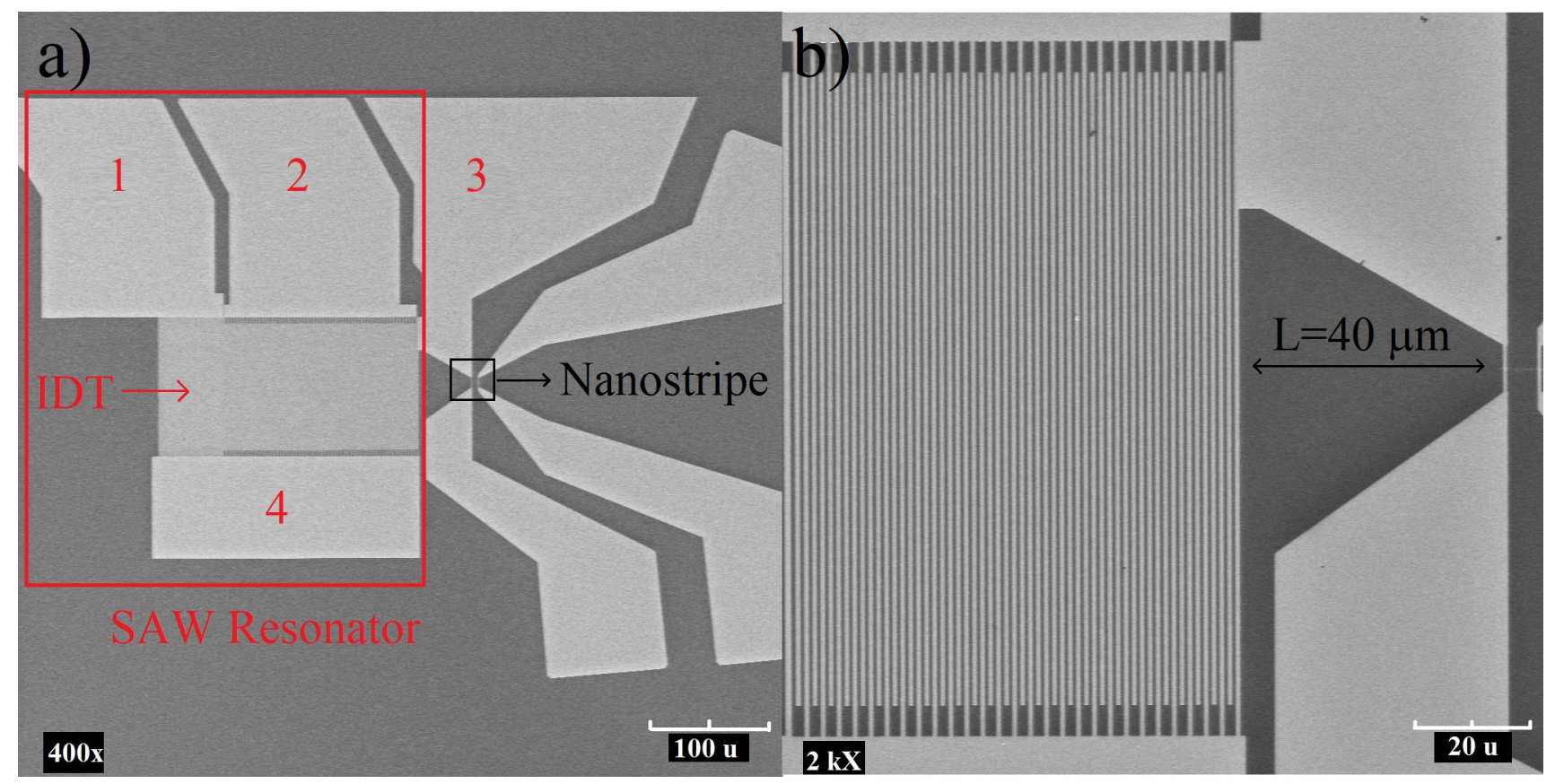

FIG. 48: a)SEM image of the complete device. Left half is the SAW resonator with the IDT and its contacts. In the half right the side contact pads of the nanostripe are visible. b) Close-up SEM image of the IDT and the nanostripe.

The structure of the SAW resonator is $C r(20 \mathrm{~nm}) / A u(120 \mathrm{~nm})$ and it is identical to the structure of the nanostripe pads. The resonator performance depends on its adhesion to the piezoelectric layer $(\mathrm{ScAlN})$ and $\mathrm{Cr}$ is the most suitable material for this task. In the case of $\mathrm{Au}$, this metal is chosen for its good quality at the emission of SAW's and also because the fact that the SAW resonator and the contact pads of the nanostripe have the same structure saves one lithography step.

The Ni nanostripes (presented in figure 49) dimensions are $10 \mu m \times 400 \mathrm{~nm}$ x $35 \mathrm{~nm}$ and its structure over the ScAlN piezoelectric layer is $C r(4 n m) / N i(35 n m) / P t(2 n m) . C r$ is chosen as a buffer layer of the $\mathrm{Ni}$ nanostripes, because of its optimal adhesion. The thickness of the deposited Cr layer is optimized in order to be the minimum necessary for the nanostripe to not to lift off. The nanostripes are triangular-pointed at both ends in order to supress the formation of flux-closure domains and they are connected to the pads by two 600 nm-thick current lines. 


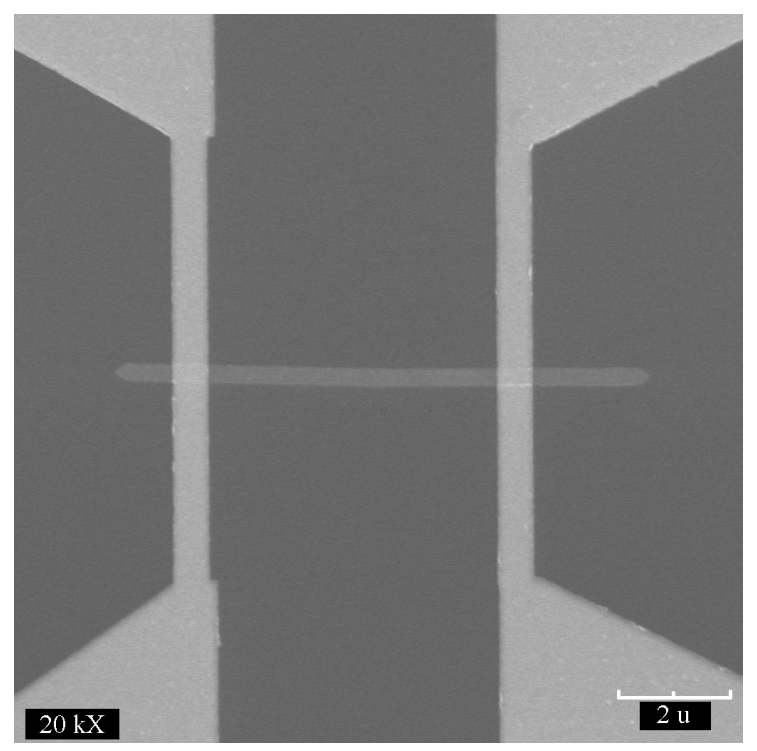

FIG. 49: SEM image of the Ni nanostripe.

In the case of $\mathrm{FeCoB}$ nanostripes, the design of the device is modified. Originally the geometry of the FeCoB nanostripes were identical to the Ni ones, however the degradation observed in FeCoB nanostripes with that geometry forced us to change the width. Apart from the width, the previous description for the Ni nanostripes applies for the FeCoB ones. In figure 50, a SEM image of one of the FeCoB nanostripes fabricated through this thesis is presented, whose dimensions are $10 \mu m \times 2 \mu m \times 35 \mathrm{~nm}$.

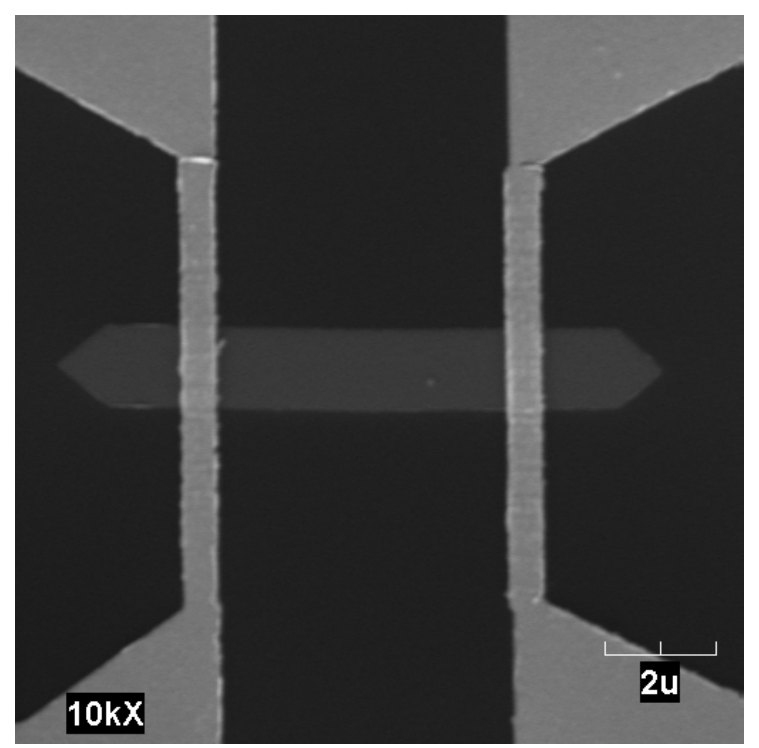

FIG. 50: SEM image of the FeCoB nanostripe. 


\section{B. SAW Resonator Characterization}

The characterization of the IDT of the SAW resonator is performed by measuring its properties of reflectivity and transmission of electrical signals. This is carried out by measuring the S-parameters, which characterize the performance of the device under the application of $\mathrm{RF}$ and microwave frequencies in terms of incident and reflected waves. Only the $S_{11}$ coefficient is measured for the characterization of our devices and it can be represented in two forms. In the first place, the $\mathrm{dB}$ representation of $S_{11}$ is known as the return loss, which is the ratio in $\mathrm{dB}$ between the amplitudes of the reflected and the incident signal. However if a more accurate description of the reflectivity is needed, it is necessary to take into account the complex nature of the parameter $S_{11}$, since it has amplitude and phase. This complexity can be displayed by the Smith Chart.

\section{SAW Resonator Reflected power.}

The description of the SAW resonator is performed by measuring the $S_{11}$ parameter, which indicates the ratio between the amplitudes in $\mathrm{dB}$ of the reflected and incident $\mathrm{RF}$ signals applied to the resonator in the frequency domain. A typical measurement of a $S_{11}$ parameter in a fabricated device is shown if figure 51.

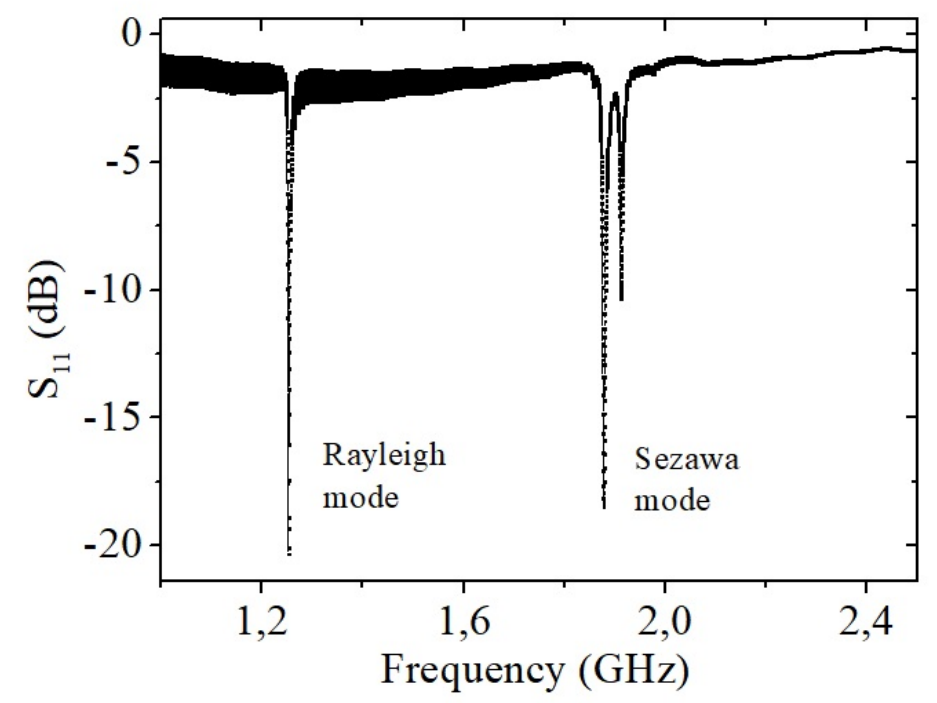

FIG. 51: Typical $S_{11}$ measurement of a SAW resonator with a wavelength $\lambda=1.4 \mu \mathrm{m}$ and a layer thickness of $2 \mu m$. 
The two main resonance peaks that can be observed correspond to the Rayleigh and Sezawa modes. The resonance frequencies $\left(f_{\text {Res }}\right)$ are dependent not only on the wavelength of the resonator, but also on the thickness of the piezoelectric layer below (ScAlN). The frequency of the fundamental modes depends on the relation between wavelength and the speed of the wave on the piezoelectric layer, which finally depends on the thickness of the layer. During this work the thickness of the layer is kept as a constant value for all samples $(2 \mu \mathrm{m})$, so that the frequency of the Rayleigh mode does not change. However, as the layer thickness is not constant throughout the entire sample, the resonance frequencies may shift slightly towards either higher or lower frequencies. Therefore all resonators need to be measured individually.

Besides from the two fundamental modes, the reflected power presents a series of repetitive resonances. These resonances are bulk acoustic waves that propagate in a vertical direction towards the substrate. $S_{11}$ shows a succession of resonances every $8 \mathrm{MHz}$ as shown in figure 52. This effect is due to the vibration of the Silicon substrate beneath the ScAlN layer [Aliev, 2012].
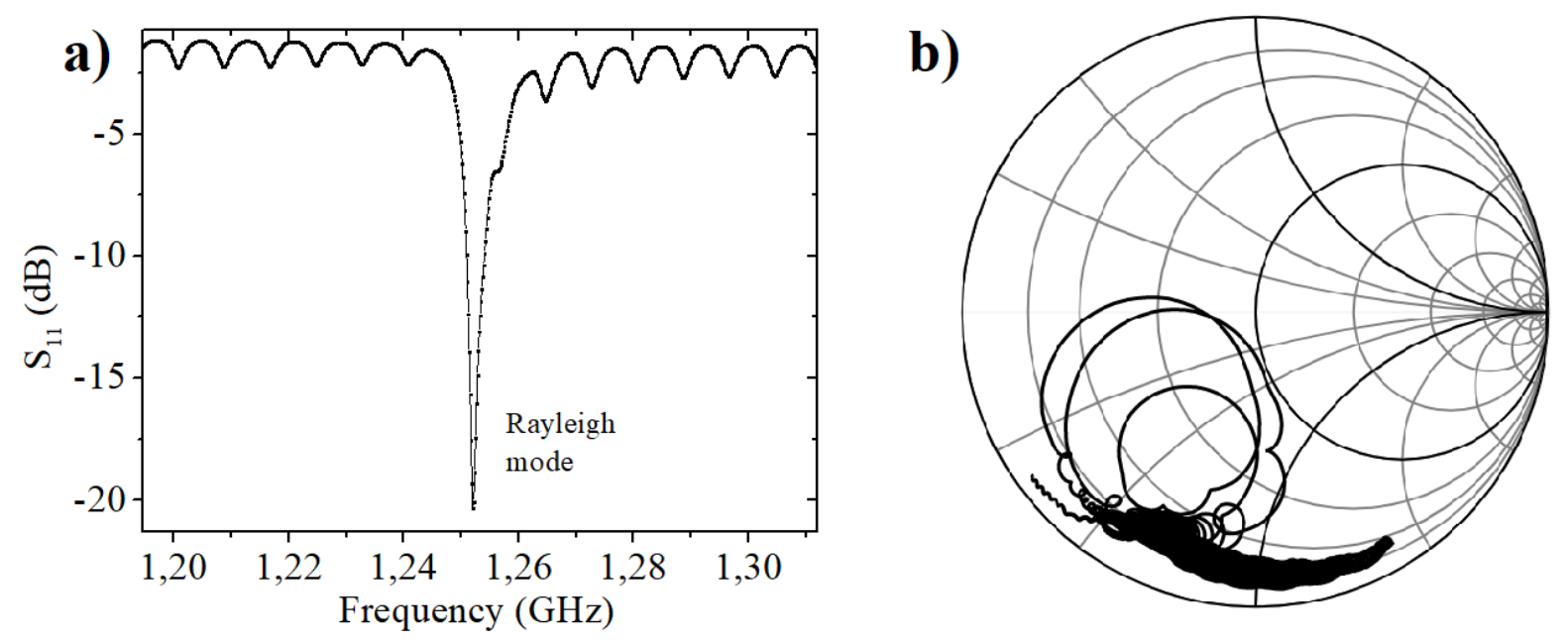

FIG. 52: a) Close up of the Rayleigh mode of the resonator at $1.25 \mathrm{GHz}$. The succession of resonances due to the Silicon can be observed each $8 \mathrm{MHz}$. b) Smith chart measured of the SAW resonator. Two big loops represent the two fundamental modes, Rayleigh and Sezawa, whereas the minor loops represent the resonances in the bulk due to the silicon substrate. 
The succession of resonances along the whole range of frequencies can also be observed in the Smith chart in figure $52 \mathrm{~b}$ ). The loops in the Smith chart represent the resonances that are seen as peaks in the $S_{11} \mathrm{~dB}$ representation.

\section{Nanostripes Characterization}

The characterization of $\mathrm{Ni}$ and $\mathrm{FeCoB}$ nanostripes is carried out by the measurement of the anisotropic magnetoresistance (AMR). In order to determine the magnetization state of the nanostripe, we measure its resistance while a DC current is flowing through it. The resistance of the nanostripe depends on the angle between the magnetization and the DC current $(\phi)$ according to the formula $R=R_{\perp}+\Delta R \cos ^{2} \phi$ ([McGuire, 1975]). In order to obtain a reasonably large signal to noise ratio, materials with large AMR effects would be preferred. However, since we need materials with large magnetostrictive coefficients, we chose $\mathrm{Ni}$ and FeCoB.

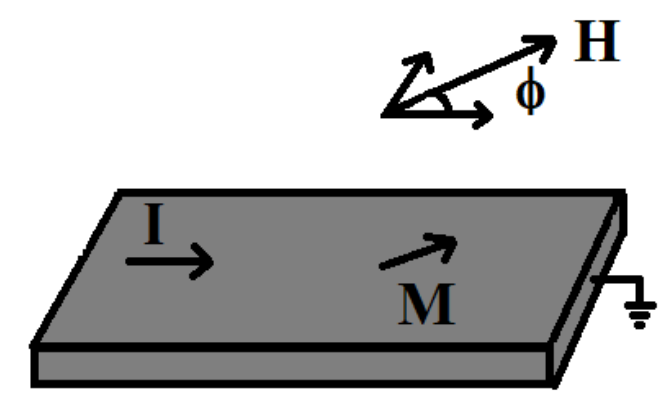

FIG. 53: Definitions of the directions of current flow (I), magnetization (M) and applied magnetic field $(\mathrm{H})$. At high enough magnetic fields, magnetization lies parallel to $\mathrm{H}$.

The AMR effect is typically large in Ni-based metals. Magnetostrictive materials gives rise to magnetic irregularities in nanostripes, such as random localized pinning sites, through magneto-elastic effects since their magnetic properties are sensitive to local strains or stresses. The AMR effect measured in $\mathrm{Ni}$ is typically $1-1.5 \%$, however is much smaller 
in $\mathrm{FeCoB}, 0.1-0.3 \%$

Definitions of the directions of current flow $(I)$, magnetization $(\vec{M})$ and applied magnetic field $(\vec{H})$ are shown in figure 53. The maximum applied magnetic field reached in our setup is 1400 Oe, large enough to saturate the magnetization in $\mathrm{Ni}$ and FeCoB. Thus, at saturation the magnetic field direction coincides with the magnetization direction of the nanostripe. Here, zero degree indicates that the direction of the applied magnetic field and that of the current are parallel.

\section{AMR in Ni Nanostripes}

As evident from figure 54 the device resistance reaches a minimum when the current flows perpendicular to the magnetization direction which is typical in Ni-based systems. Therefore, by measuring the nanostripe resistance, we can infer the amount of magnetization that misaligns from the DC current flow direction.

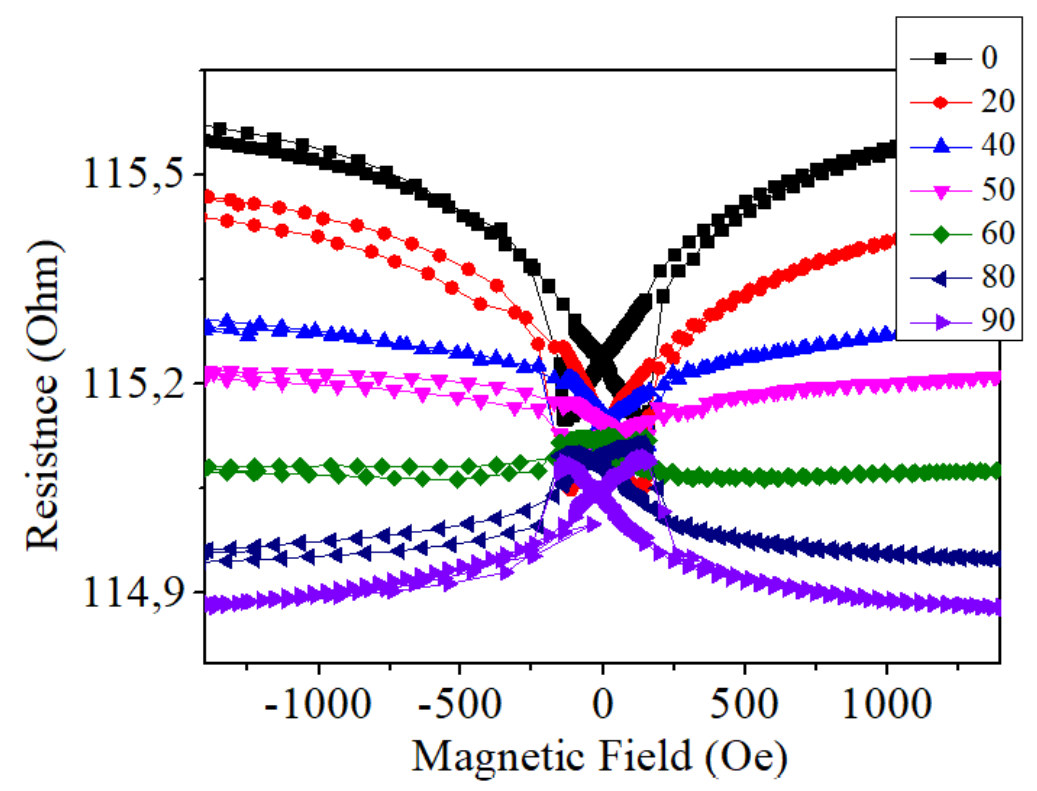

FIG. 54: Magnetoresistance measurements of a $35 \mathrm{~nm}$ thick Ni nanostripe. Different curves are plotted with different angles between the applied DC current and the external magnetic field. At saturation, the resistance is maximum when magnetization lies parallel to the DC current and minimum when both are perpendicular 
Figure 54 shows an example of AMR measurement of a $35 \mathrm{~nm}$-thick Ni nanostripe. For now we only need to look at the resistance values where magnetization is saturated at high fields, since at these fields we assume magnetization $\vec{M}$ and $\vec{H}$ are parallel. The value of the AMR effect is taken as the percentage difference between the maximum when $\vec{M}$ and $\vec{H}$ lie parallel and when they are perpendicular. In the example presented in figure 54 $\mathrm{AMR}=0.8 \%$. As stated before, the typical values for $\mathrm{AMR}$ in $\mathrm{Ni}$ measured are in the range of $1-1.5 \%$, thus, the AMR effect in the nanostripe of the example is slightly small. We plot the values of saturated magnetization resistance as a function of the angle between the applied DC current and the magnetization (Magnetic field). The device resistance scales with the angle $\phi$ as:

$$
R=R_{\perp}+\Delta R \cos ^{2} \phi
$$

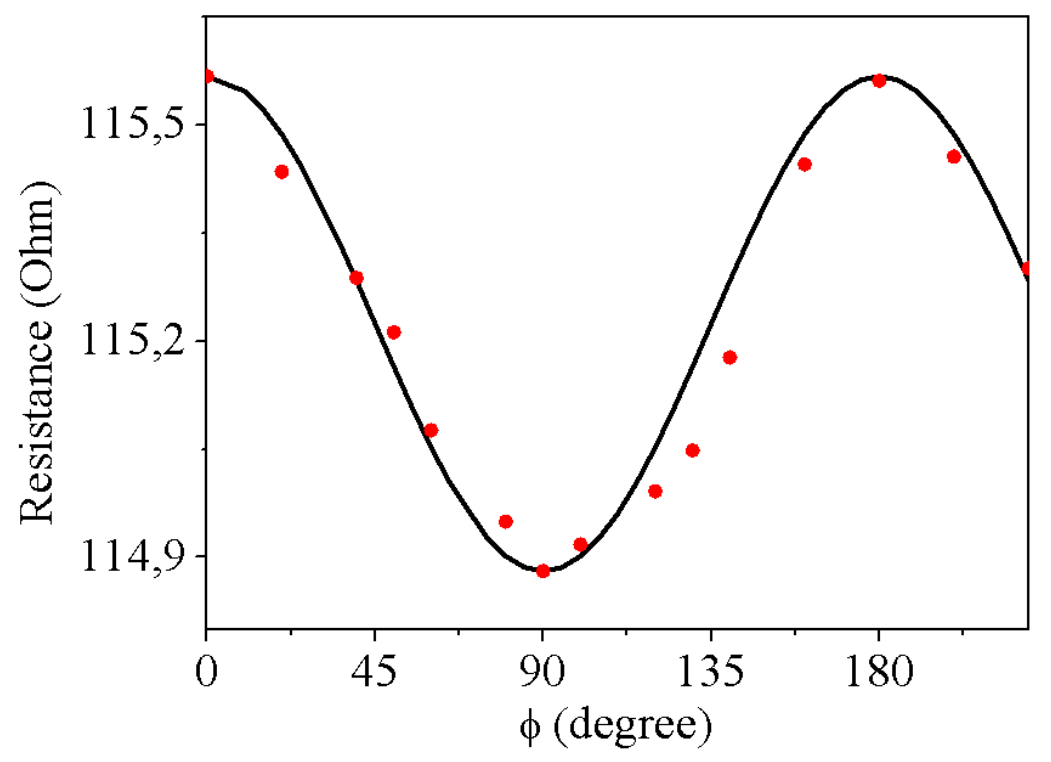

FIG. 55: Dependence of the resistance of a $35 \mathrm{~nm}$ thick Ni nanostripe on the relative angle between the applied magnetic field and current direction. Zero degree indicates that the directions of the applied magnetic field and that of the current are parallel. The applied magnetic field is 1400 Oe, large enough to saturate the magnetization.

In equation (16) $\Delta R=R_{\|}-R_{\perp}$, where $R_{\perp}$ and $R_{\|}$are the resistance values when the magnetization lie perpendicular and parallel to the DC current flow direction respectively. 


\section{AMR in FeCoB nanostripes}

AMR measurements are also performed in $\mathrm{FeCoB} 35 \mathrm{~nm}$ thick nanostripes. Similarly to the case of the $\mathrm{Ni}$, the resistance reaches a minimum when the magnetization $(\vec{M})$ lies perpendicular to the direction of the DC current flow and maximum when both $\vec{M}$ and $I$ are parallel. The AMR effect in this material is considerably smaller than the AMR in Ni. $\mathrm{AMR}$ effect in $\mathrm{FeCoB}$ is obtained by the difference in percentage of the resistance values in saturation when the magnetic nanostripe lies parallel and perpendicular to $\vec{H}$. In the example presented in figure 56, the AMR effect is $0.15 \%$, which is in the range of the typical values measured 0.1-0.2\%. AMR measurements performed on FeCoB nanostripes show that they have an anisotropy direction parallel to the long axis.

a)

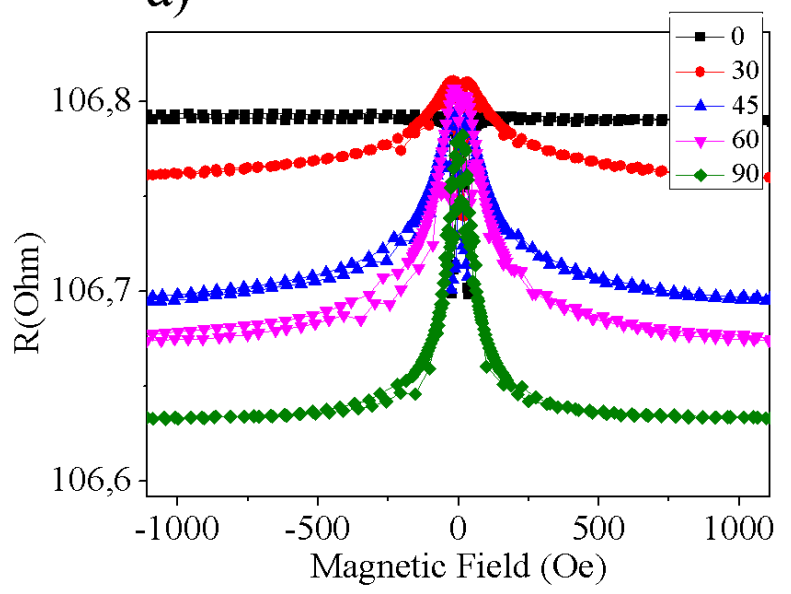

b)

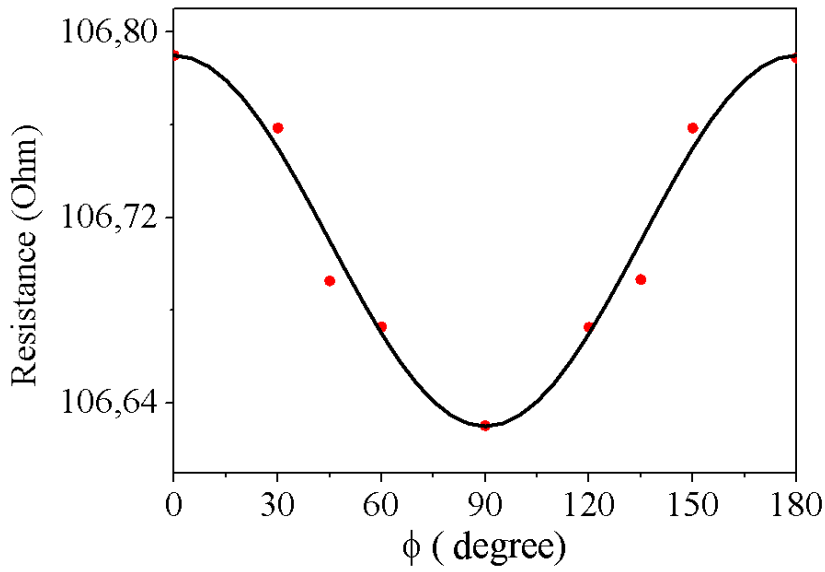

FIG. 56: a) AMR measurements of a $35 \mathrm{~nm}$ thick FeCoB nanostripe. Different curves are plotted with different angles between the applied DC current and the external magnetic field. b) Dependence of the resistance of the nanostripe on the relative angle between the applied magnetic field and current direction. Resistance values are taken at an external field of 1400 Oe. 


\section{Magnetic Structure of Ni nanostripes}

The magnetic structure of the nanostripes is mainly infered by magnetoresistance (AMR) measurements. The experimental setup is shown in figure 72 where the nanostripes are placed parallel to an external magnetic field. As stated in Chapter V, Ni nanostripes are divided in two sets, since throughout this thesis, the nanostripes were grown out of two different Ni targets by sputtering deposition. The differences between nanostripes are mainly crystalline (grain size), which result in different magnetic properties. Nevertheless, both sets of nanostripes present qualitatively identical magnetic structures.

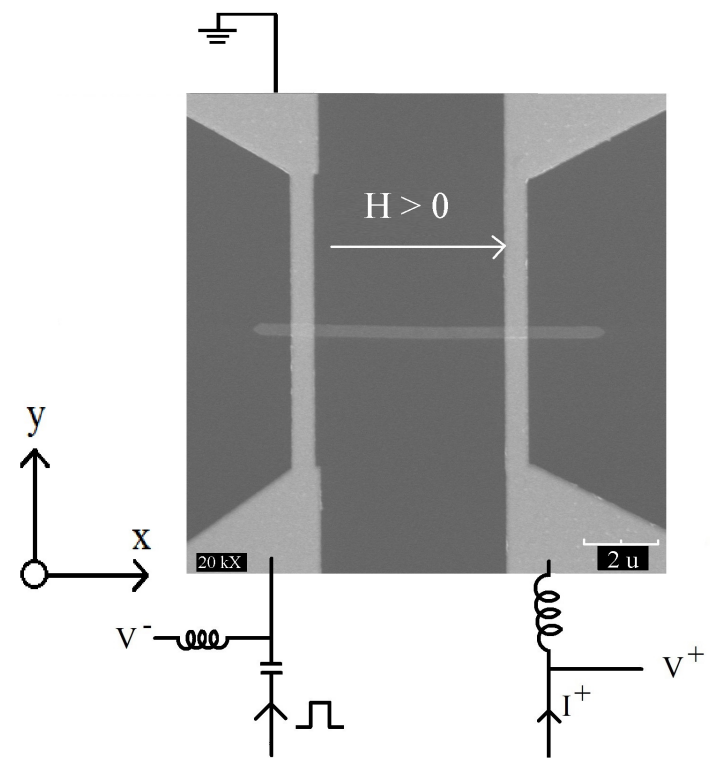

FIG. 57: Close up of the Ni nanostripe and the setup for magnetoresistance measurements. The direction of the external magnetic field applied is depicted by the arrow

Magnetoresistance measurements are performed in the first set of devices, whose nanostripes have a grain size $D_{1}=11 \mathrm{~nm}$. Magnetoresistance curves are shown in figure 58, where it can be observed how from saturation to low fields, the resistance signal decreases as the magnetic field approaches to zero. This is a clear consequence of an averaged uniaxial anisotropy whose direction does not match with the long axis of the nanostripe (DC current flow direction). Because of the polycrystalline structure of the nanostripe, every crystal has its own anisotropy direction, therefore it is expected each crystal magnetization to misalign from the DC current flow direction as the magnetic field does not force the magnetization 
along the long axis of the nanostripe. Thus, a decrease in resistance occurs. As seen in figure 58 the resistance of this nanostripe decreases down to $-0.3 \%$ at zero applied field. Further AMR measurements confirms the anisotropy direction at $45^{\circ}$ away from the long axis of the nanostripe.

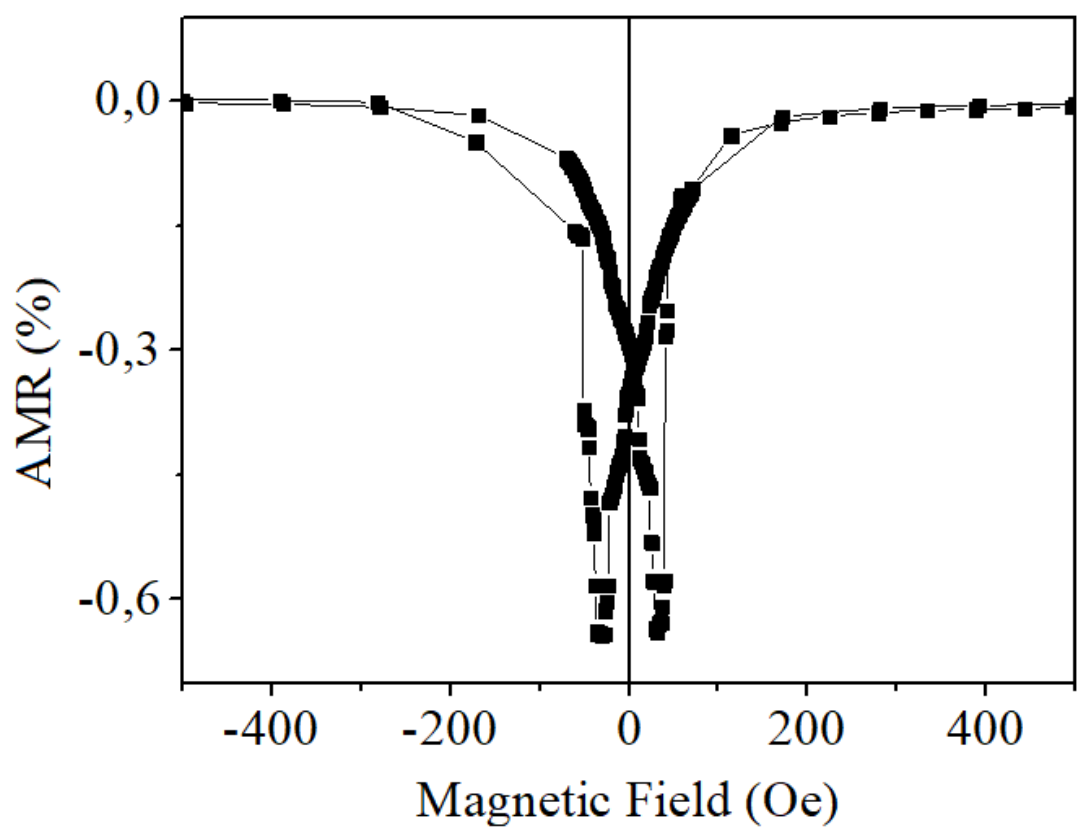

FIG. 58: AMR measurement of the Ni nanostripe of the set \#1 whose grain size is $11 \mathrm{~nm}$. Magnetic field is applied parallel to the long axis of the nanostripe.

The AMR signal has two main contributions which depend on the angle between magnetization and the DC current flow. We can consider the first contribution coming from the rotation of the magnetic moments. As the anisotropy direction is misaligned from the long axis of the nanostripe, it is infered that, the decrease of the applied field from saturation to low fields, generates a rotation of the magnetic moments away from the $\mathrm{x}$ direction (see figure 57), decreasing the resistance. It is expected that the rotation of the magnetic moments is a reversible process. At some point in the AMR loop, a non-reversible transition is expected to occur, typically associated to a domain wall (DW) nucleation [Hong, 1998], which is a magnetic entity that naturally misaligns the magnetization out of the long axis of the nanostripe. The magnitude of the decrease in resistance that would generate the nucleation of a DW $\left(\Delta R_{D W}\right)$ depends on the AMR of Ni. Since AMR in these nanostripes 
are $1-1.5 \%$, the value of $\Delta R_{D W}$ is expected to be between $0.05-0.2 \Omega$.

In order to identify the magnetic field when the AMR loop becomes irreversible, we perform minor loops measurements. In these, the magnetization of the nanostripe is saturated in one direction, then, $\vec{H}$ is decreased down to a magnetic field around zero, which could switch the initial direction of the magnetic field. After that, $\vec{H}$ is increased in the initial direction until saturation. Minor loops studies show if the magnetization is reversible and at which field reversibility breaks. When this occurs and hysteresis appears, typically is the sign of the presence of a DW. The objective of these studies is to find when the resistance signal starts to show hysteresis, therefore, a non-reversible transition has occured (typically a DW nucleation). We perform minor loops measurements for the first set of nanostripes, whose grain size is $D_{1}$ with an anisotropy direction $45^{\circ}$ away from the long axis of the nanostripe. Figure 59 shows minor loops from positive saturation to 3 Oe and -5 Oe.

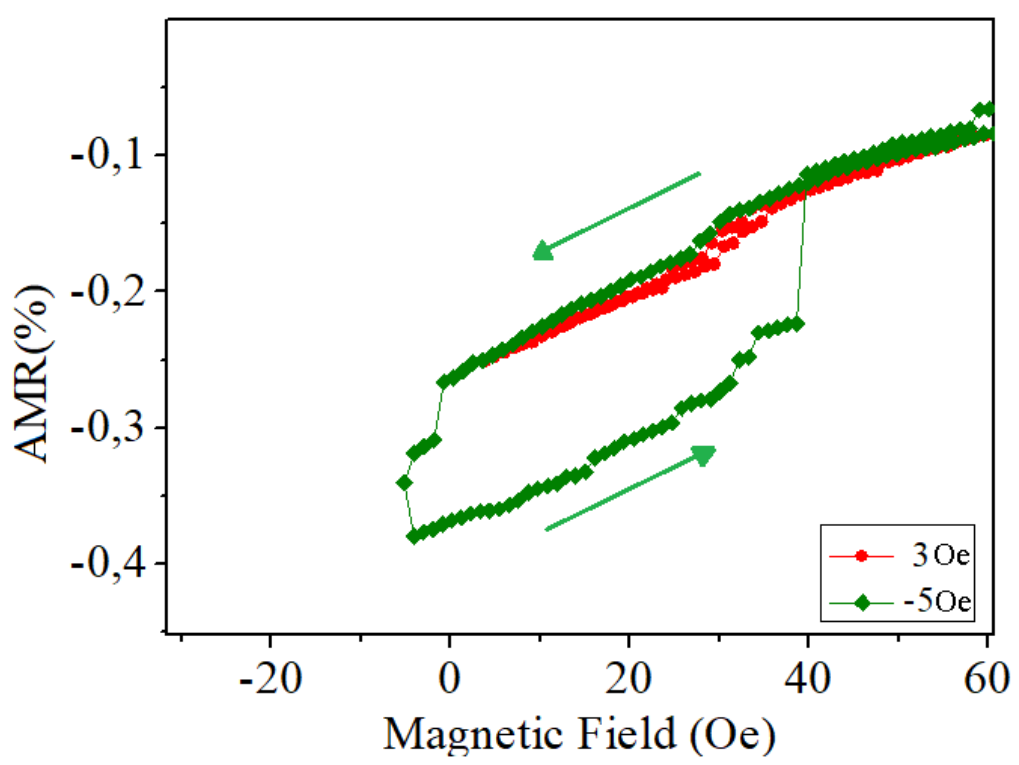

FIG. 59: Comparison of minor loops down to two different magnetic fields.

Two curves are plotted, one of them (red curve) reaches down to 3 Oe from positive saturation. It can be observed that this curve shows reversibility i.e. magnetization states are the same independently the external field is being either decreased or increased. The second curve (green curve), reaches -5 Oe. As it can be seen hysteresis happens due to 
a drop of the resistance value at low negative fields. Besides from the drop in resistance, there is another jump in resistance at 40 Oe, which matches the coercive field observed in the AMR complete loop (figure 58). Several measurements were carried out and the field range where the minor loop turned non-reversible was between -1 and -5 Oe.

Nanostripes grown out of the second Ni target with grain size $D_{2}=18 \mathrm{~nm}$ are measured. Magnetoresistance measurements (figure 60) show that the preferential magnetization direction in the nanostripe misaligns with the long axis, which can be seen by the decreasing in the resistance signal from saturation to low magnetic fields. At zero field the resistance is decreased down to $-0.6 \%$. Further measurements with different angles of application of $H_{\text {ext }}$ shows that the anisotropy direction is $55^{\circ}$ away from the long axis.

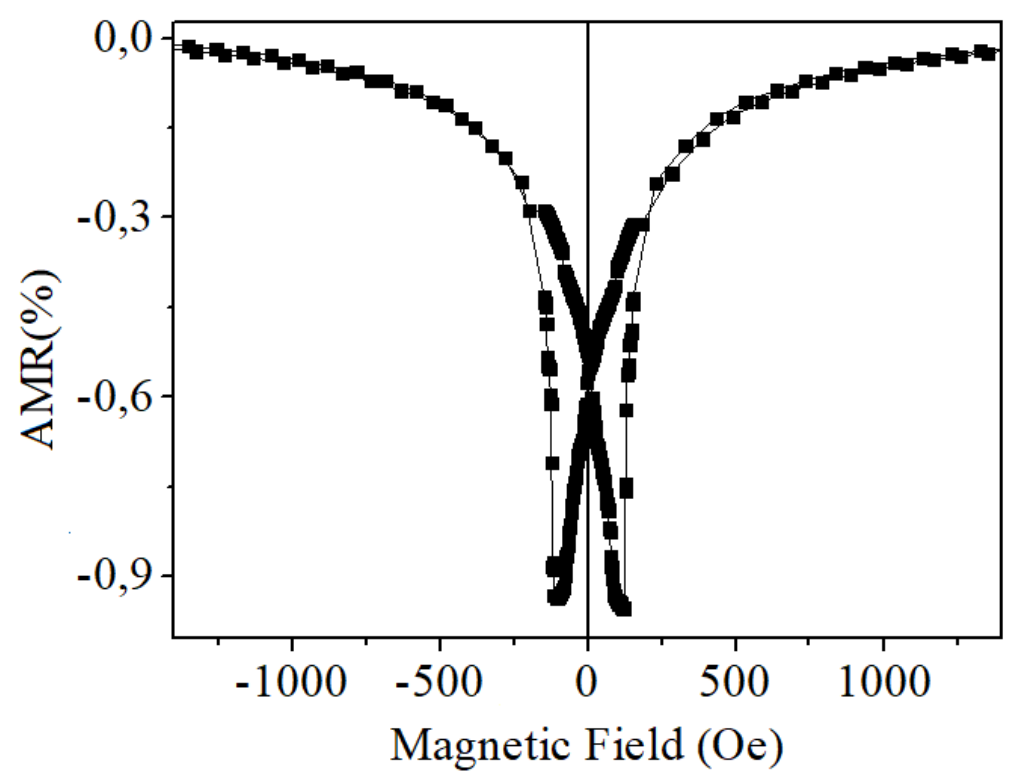

FIG. 60: AMR measurements of a Ni nanostripe with an average grain size of $18 \mathrm{~nm}$.

Qualitatively the magnetoresistance measurement in this nanostripe is analogous to the one presented in figure 58. We now perform minor loops on this nanostripe with a grain size $D_{2}$. Results are presented in figure 61 and they show differences from the previous nanostripe. Once the external field applied switches its direction hysteretic behaviour is present and gradually enhances. As it can be seen from both minor loops, the hysteretic behaviour is achieved gradually, which makes it difficult to identify the precise magnetic 
field where a non-reversible transition occurs. This results are better understood when MFM measurements and the simulations are presented.

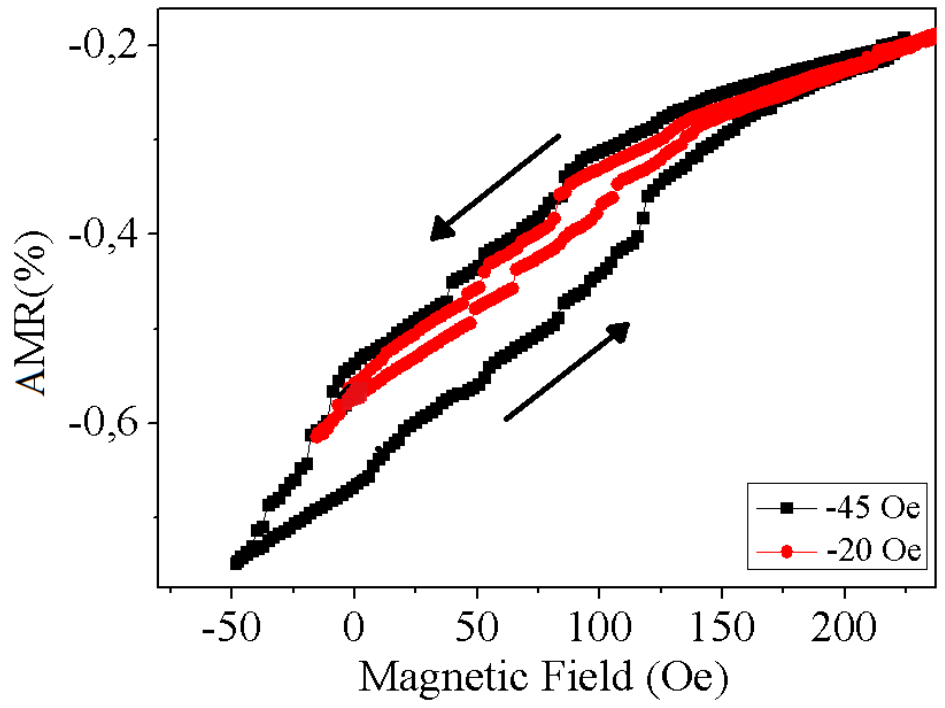

FIG. 61: Comparison of minor loops down to two different magnetic fields in a nanostripe with an average grain size of $18 \mathrm{~nm}$.

In order to achieve a better understanding of the magnetic structure of the Ni nanostripes, magnetic force microscopy (MFM) measurements are carried out in absence of applied field $(H=0)$. In figure $62 \mathrm{a})$ and $\mathrm{b}), \mathrm{AFM}$ and MFM images of the Ni nanostripe at remanence are presented. Different shades in the MFM image indicate the presence different magnetic domains. We observe how the Ni nanostripe is divided in alternated domains, whose magnetization is expected (due to the decrease of the magnetoresistance at remanence) to point away from the long axis of the nanostripe. Furthermore, Mumax micromagnetic simulations are carried out by the Departament of Fisica Aplicada of University of Salamanca on a nanostripe with dimensions of $35 \mathrm{~nm} \times 10 \mu \mathrm{m} \times 400 \mathrm{~nm}$ in order to describe the magnetization reversal process in these nanostripes. We assume a small uniaxial anisotropy of $K=$ $6.0 \cdot 10^{3} \mathrm{~J} / \mathrm{m}^{3}$ in the y direction, a saturation magnetization of $M_{s}=2.38 \cdot 10^{5} \mathrm{~A} / \mathrm{m}$ and exchange constant of $A=1.05 \cdot 10^{-11} \mathrm{~J} / \mathrm{m}$. The polycrystalline grain structure is modelled via a Voronoi tessellation of the system in polygonal regions of average diameter of $D=20$ $\mathrm{nm}$. The external field was ramped in steps of $1 \mathrm{mT}$, maintaining each field for a time interval $t=100 \mathrm{~ns}$ and with $\alpha=0.01$ as damping constant. Snapshots of the process are presented in figure 63. Simulation starts with the application of a large external magnetic 
field $\left(H_{e x t}\right)$ which saturates the magnetization, forcing it to point parallel to the $\mathrm{x}$ direction (1), at this point the resistance is maximum.
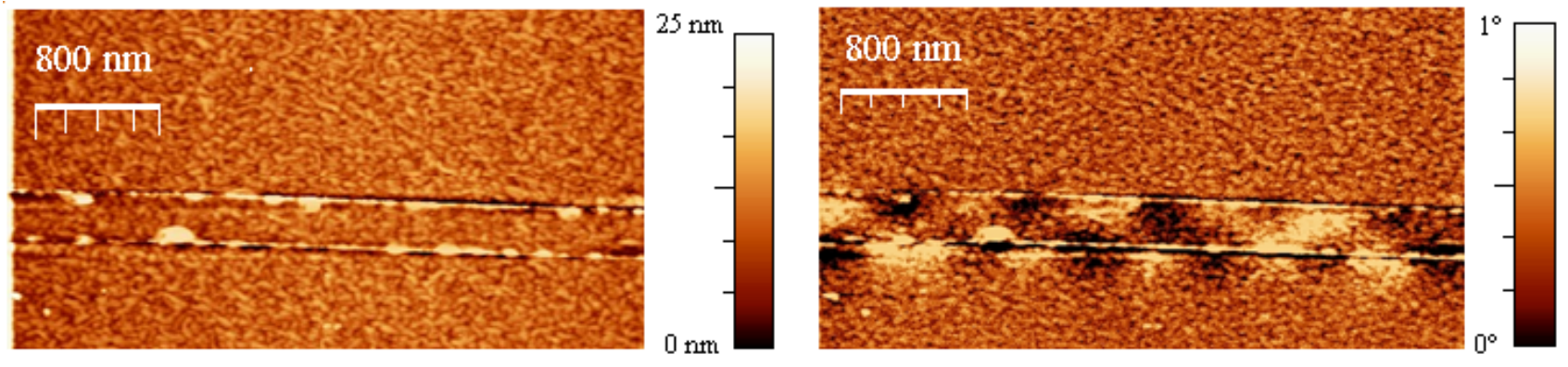

FIG. 62: a) AFM image and b) MFM image of a Ni nanostripe measured at $H=0$.

As $H_{e x t}$ is reduced from saturation, the system breaks into domains oriented along the y direction separated by DW's oriented in the $\mathrm{x}$ direction which decreases the resistance of the nanostripe (3). After $H_{\text {ext }}$ switches its direction, eventually a non-reversible event occurs, where two or more domain walls collapse with each other and/or a new domain oriented in the $-\mathrm{x}$ direction arises (4). As $H_{\text {ext }}$ keeps increasing in the opposite direction to the initial, the new domain is expanded by the pinning of the DW's in different pinning sites (4-5). Finally, a further increase of $H_{\text {ext }}$ saturates the magnetization (7).

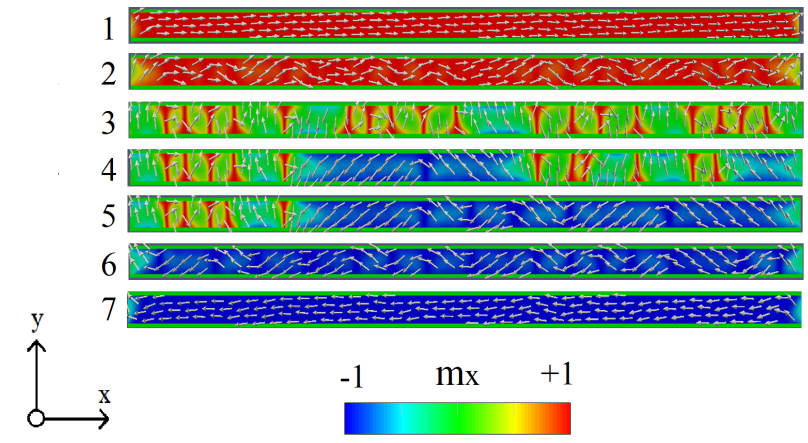

FIG. 63: Images from micromagnetic simulations showing the reversal process in a Ni polycrystalline nanostripe.

Simulations show that x-oriented DW's appear during the reversible part of the AMR loop and they separate the domains oriented in the y direction. When a non-reversible transition occurs, this is related to the emergence of a domain oriented in the $-\mathrm{x}$ direction, opposite to the initial $\mathrm{x}$ direction. 


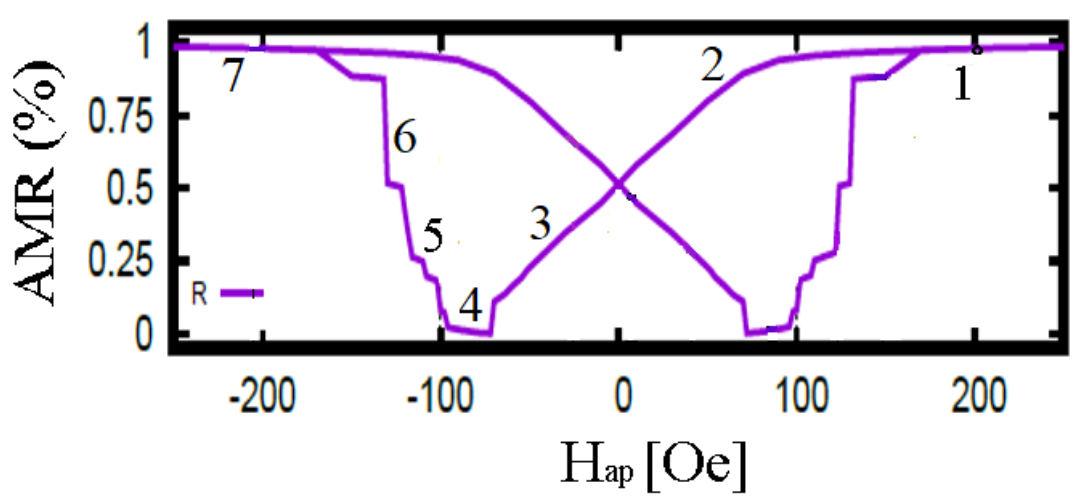

FIG. 64: Simulated magnetoresistance in the Ni polycrystalline nanostripe.

\section{E. Coercive Fields}

The coercive fields is one of the features affected by crystalline properties and therefore susceptible of changing in different samples with different grain sizes. In our magnetoresistance (AMR) measurements, we identify the coercive fields by the fields where the minima AMR signal is found. Two regimes can be found in the literature that relate the grain size of a magnetic nanostructure and their coercive fields. First regime goes from large grain sizes down to sizes where the grain are comparable to the typical DW width of the material, whereas the second regime covers grain sizes below that value. The typical DW width is given by the equation :

$$
\delta_{w}=\pi \sqrt{A / K_{N i, b u l k}}
$$

In $\mathrm{Ni}$, with an anisotropy constant of $K_{b u l k, N i}=5 \cdot 10^{3} \mathrm{~J} / \mathrm{m}^{3}$ and an exchange constant $A=1.05 \cdot 10^{-11} \mathrm{~J} / \mathrm{m}$, the DW width is $\delta_{w}=143 \mathrm{~nm}$. The studies of coercive fields in polycrystalline Fe and Ni carried out by [Loffel, 1998], show a maximum when the grain size of the magnetic sample is a similar dimension of the typical DW width. In the range of a grain size dimensions larger than $\delta_{w}$ an increase in coercivity with decreasing grain size, approximately following a $\mathrm{H} \sim 1 / \mathrm{D}$ behavior, is observed in polycrystalline materials. This is explained by DW pinning at grain boundaries that becomes progressively more efficient as the volume fraction of grain boundaries increases. Below the range of $\delta_{w}$, there is a steep decrease of coercive field towards smaller grain sizes. This decrease can be explained by the random-anisotropy model. In consolidated materials, exchange coupling between 
neighboring grains is possible and may overcome the magnetocrystalline anisotropy of each grain. This exchange coupling results in a reduced effective anisotropy, which represents an average over the magnetocrystalline anisotropies of the correlated grains. For a random orientation of the anisotropy axes of the grains the effective anisotropy is reduced by a factor of $1 / \sqrt{N}$, where $\mathrm{N}$ is the number of the magnetically correlated grains. Consequently, one expects a decrease of the anisotropy energy and therefore also a decrease of the coercive field with decreasing grain size. For our first set of nanostripe samples, whose grain size is 11 $\mathrm{nm}$, which is considerably smaller than the second set of Ni nanostripes. The coercive fields for this set of samples are \pm 40 Oe, shown in magnetoresistance (AMR) measurements in figure 65 a).

a)

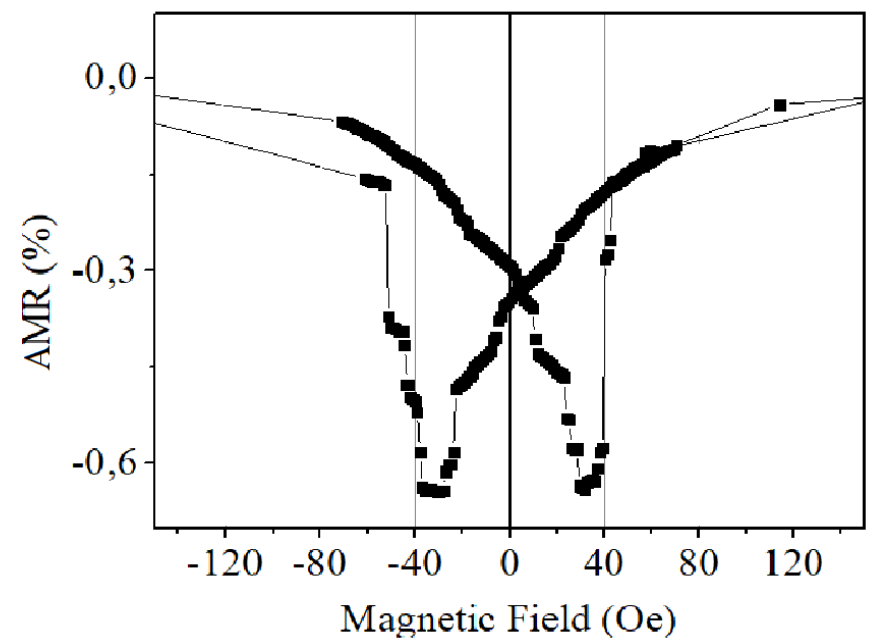

b)

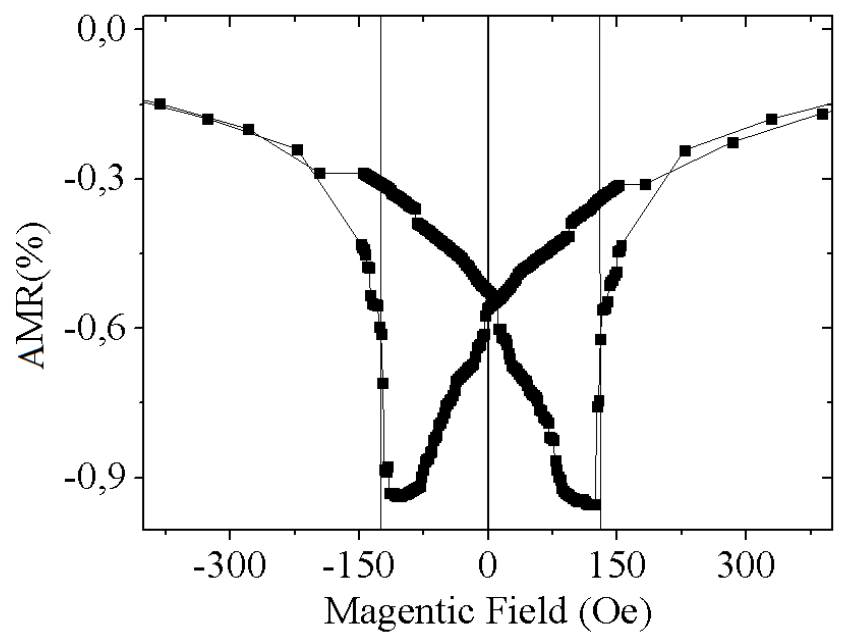

FIG. 65: a) Close up of the magnetoresistance measurements of Ni nanostripes of set \#1 of samples. Coercive fields are \pm 40 Oe. b) Close up of the MR measurements of Ni nanostripes of set \#2. Coercive fields are \pm 120 Oe.

For nanostripes grown out of the second target, the grain size dimensions, obtained by the X-ray diffractograms, is $18 \mathrm{~nm}$, which is larger than the previous one. The MR curves obtained are shown in figure $65 \mathrm{~b}$ ). The coercive fields for this set of nanostripes are around \pm 120 Oe which are clearly larger than in the previous set of nanostripes. As we find the grain size of our samples in a range below the dimensions of $\delta_{w}$, it is expected that coercive fields decrease with decreasing grain size of the nanostripes. 


\section{F. Experiments with pulsed local fields}

By the local field injection method one can simply apply a local magnetic field, large enough to change the magnetization direction locally. From Ampere's law, magnetic fields are generated when a current passes through a conducting wire. The larger the current, the larger the magnetic field. By reducing the size of the conducting wire, it is possible to generate a localized magnetic field around the wire. We initilly used this concept with the objective of injecting DW's in the Ni nanostripes. In figure 66 a) it is presented the setup for the injection of single current pulses.
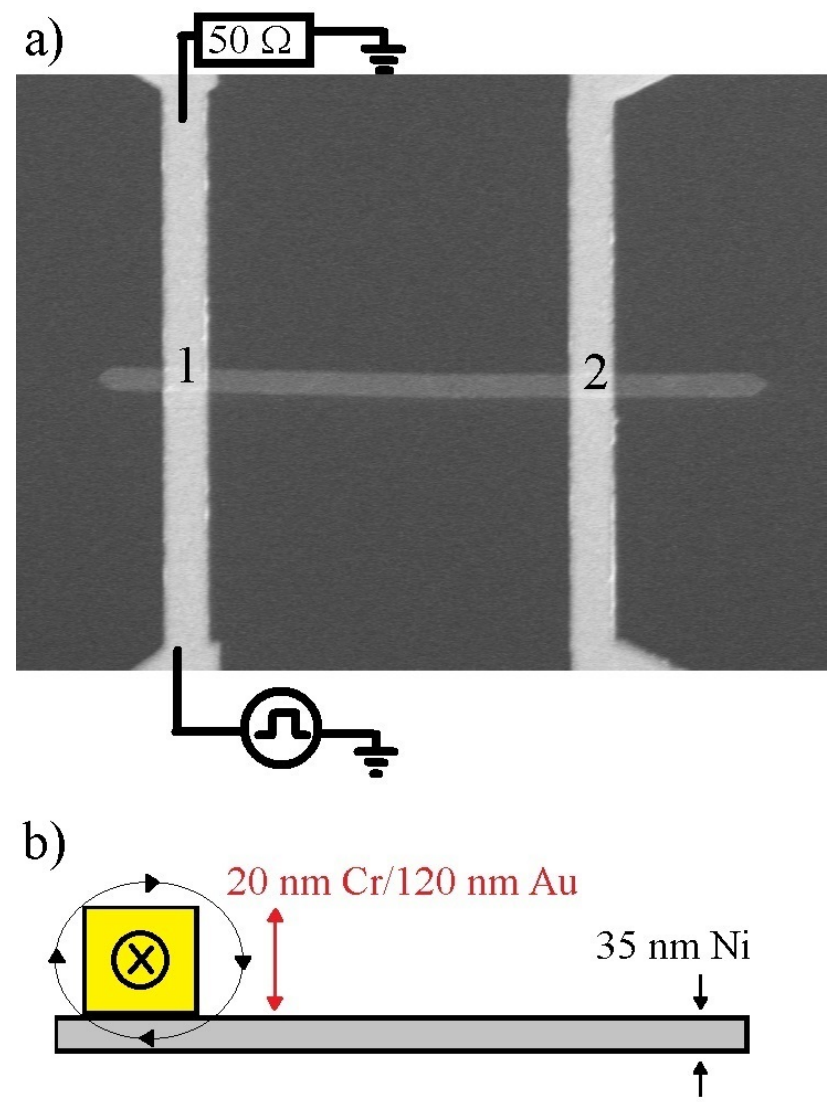

FIG. 66: a) SEM image of a Ni nanostripe (horizontal) and its electrical contacts (vertical lines). A schematics of the experimental setup is drawn on top. b) Section schematics of the local field injection method. The current pulse is applied through line 1 generating a large field in the nanostripe. 
The two vertical lines 1 and 2 are the current lines of the nanowire made of $20 \mathrm{~nm} \mathrm{Cr}$ $120 \mathrm{~nm} \mathrm{Au}$. These two lines are connected to each other via the Ni nanostripe. The width of the current line is set to $600 \mathrm{~nm}$ and the resistance is 15-20 $\Omega$. Current is passed through line 1 to generate a localized magnetic field around the line. In order to generate maximum possible field, it is necessary to pass a large amplitude current through the line. However, this may result in Joule heating which can significantly damage the sample. Thus, to avoid these effects, we use current pulses to minimize the power delivered to the line. In addition, using current pulses to generate magnetic field provides a control of timing the magnetic field application, which is impossible to do when electromagnets are used. In other words, one can time resolve the movement or nucleation of domain walls.

The procedure of injecting current pulses through the local field method starts with the application of a large magnetic field on the nanostripe in order to saturate the magnetization in one direction $\left(H_{S a t}\right)$. After that, the external magnetic field is decreased down to a low magnetic field of opposite direction, which is called injection field $\left(H_{i n j}\right)$. When this process is used for the injection of DW's in the nanostripe, it is important that the reversal process has not yet started at $H_{i n j}$ and therefore, no DW is already nucleated. At this point the current pulse is applied through the current line, which generate a local magnetic field and reverse the magnetization locally in the proximity of the current line. The resistance of the nanostripe is measured $100 \mathrm{~ms}$ before $\left(R_{1}\right)$ and after $\left(R_{2}\right)$ the pulse is applied. The difference between the resistances $\left(\Delta R=R_{1}-R_{2}\right)$ is measured. Notice that to consider a DW injection in the presented setup, the condition needed is $\Delta R>0$. The DW lowers the resistance of the nanostripe since it misaligns the magnetization with the current flow direction.

However, we have observed that at low fields the Ni nanostripes present a multidomain structure with DW's separating the alternated domains. Therefore, a drop in resistance in this experiment do not represent necessarily the injection of a DW, but the triggering of a non-reversible event associated to the nucleation of a domain oriented in the opposite direction to the initial saturating direction. In this section, $\Delta R$ is measured with the same parameters of pulse amplitude, pulse length and injection field, hundreds of times. Then, the measurements of $\Delta R$ obtained with those parameteres are arranged in a histogram table. 
We present histograms results for the first set of nanostripes, with a grain size of $D_{1}$ with an uniaxial anisotropy in the direction at $45^{\circ}$ away from the long axis of the nanostripe. In figure 67 we present the results for measurements performed at injection fields of $H_{i n j}=-1$ Oe and $H_{i n j}=-2$ Oe, with a pulse of 3 ns duration and $2 \mathrm{~V}$ amplitude.

a)

b)

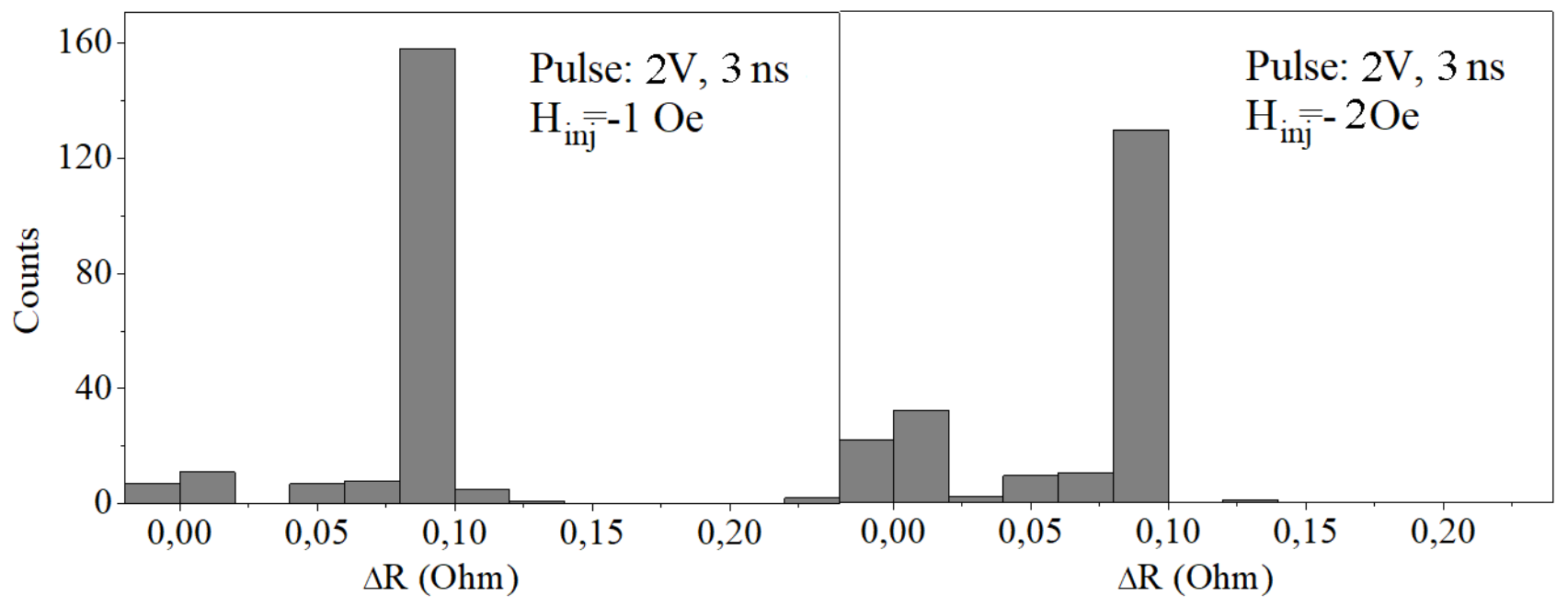

FIG. 67: Histograms of $\Delta R$ for a pulse of $2 \mathrm{~V}$ and $3 \mathrm{~ns}$ for a injection field of -2 Oe.

The pulse parameters of amplitude and duration are optimized for the measurements presented in figure 67, where the injection fields are $H_{i n j}=-1$ Oe and $H_{i n j}=-2$ Oe. These histograms show a preferential drop in resistance at $\Delta R=0.08 \Omega$, plus several counts around $\Delta R=0 \Omega$. In this case, instead of a DW nucleation, the drop in resistance is related to the triggering of a non-reversible transition in the Ni nanostripe. In order to count a successful event, we set a threshold value $(\Delta R=0.05 \Omega)$ considered the minimum value of $\Delta R$ to be equivalent to a DW nucleation. By the histograms results, we do not find probability of 1 of triggering non-reversible events, as several counts around $\Delta R=0 \Omega$ are measured. Results show that with larger injection fields, fewer events are measured that present a drop in resistance, to the point that not even one event present a drop resistance at injection fields $H_{i n j}=-15$ Oe and larger. For this sample, the probability of triggering a non-reversible event is measured for a range of injection fields and amplitudes of the current pulse. By this, we can plot a map contour (Figure 68) presenting the injection of non-reversible events probabilities as a function of these parameters. However, there are no values for these parameters where the probability of 1 is found. 


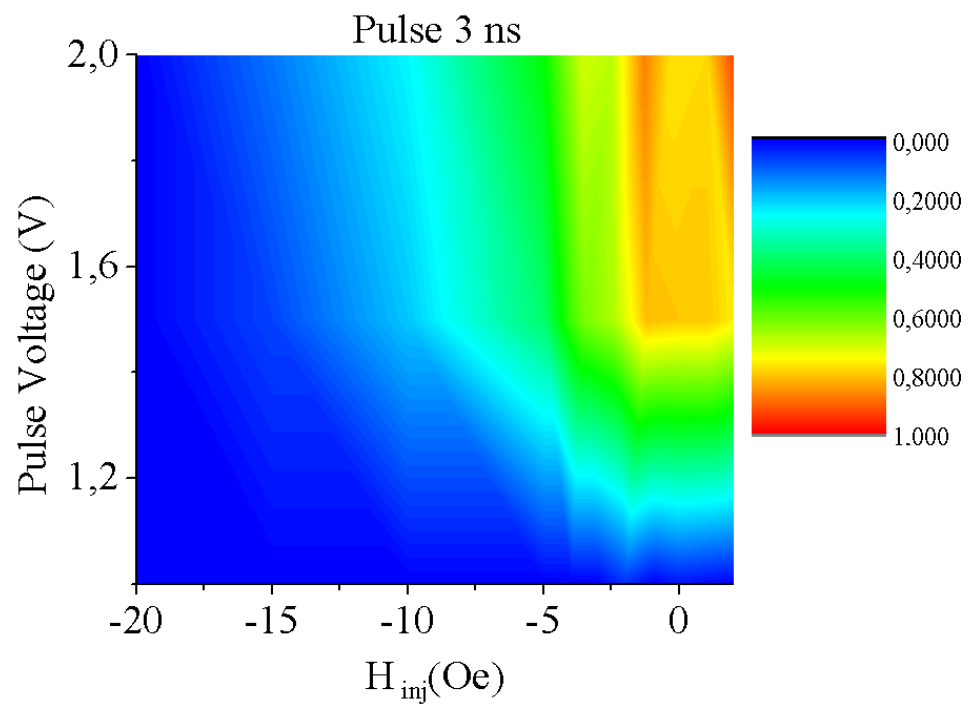

FIG. 68: Map of probability of DW injection as a function of the injection field and the amplitude of the applied pulse.

We now present the results of the injection of current pulses at low injection fields for the second nanostripe, whose average grain size is $D_{2}$. The nanostripe is saturated in the positive direction and the injection fields are $H_{i n j}=-1$ Oe and $H_{i n j}=-2$ Oe. These histograms show that $\Delta R$ counts in this case have more dispersion than in the previous nanostripes. Events are measured in a range of $\Delta R=[-0.01 \Omega, 0.15 \Omega]$ without any discontinuity. The measurements in the histograms are centered around the $\Delta R=0.05 \Omega$ value.

a)

b)

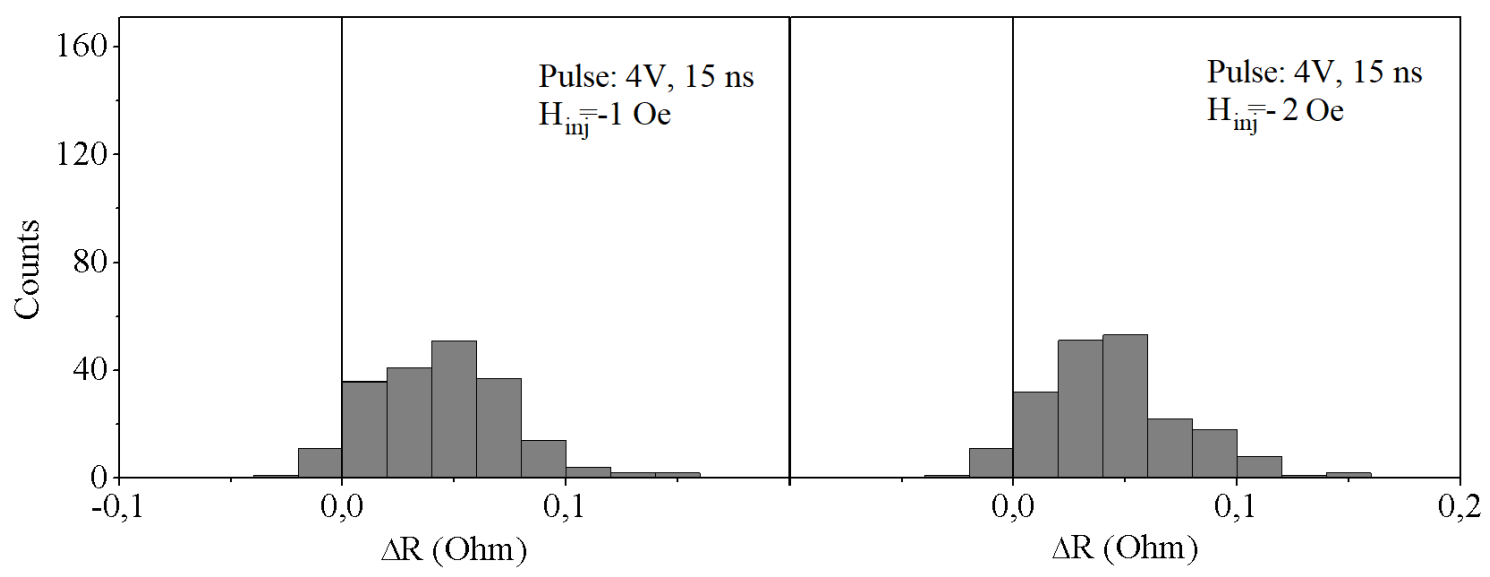

FIG. 69: Histograms of $\Delta R$ for a pulse of $4 \mathrm{~V}$ amplitude and $15 \mathrm{~ns}$ length for a injection field of -1 Oe. A line at $\Delta R=0$ is depicted for clarity. 
The histograms in both nanostripes are comparable to the minor loops measurements (figures 59 and 61). In the nanostripe with grain size $D_{1}=11 \mathrm{~nm}$, the non-reversible events occur in an abrupt manner by sudden jumps, whereas in the nanostripe with grain size $D_{2}=18 \mathrm{~nm}$, the minor loop present irreversibility in a gradual manner that indicates the non-reversible event occurs gradually.

\section{G. Conclusions}

In this chapter we have presented all the measurements performed on the straintronics devices in order to characterize them and collect all the information possible in absence of surface acoustic waves (SAW's). We start this chapter with a visual description of the devices which is formed by a SAW resonator and a ferromagnetic nanostripes with their contact pads.

The SAW resonator is the device that generates strain by the emission of SAW's. It is formed by the IDT's (interdigital transducers), which is an array of metallic bars alternatively connected to different contact pads. The resonators are characterized by the $S_{11}$ parameter, which indicates the ratio between the amplitudes in $\mathrm{dB}$ of the reflected and incident RF signals applied to them in the frequency domain. The measurements of $S_{11}$ in our SAW resonator show two fundamental modes, Rayleigh $(\sim 1.25 \mathrm{GHz})$ and Sezawa $(\sim 1.8$ $\mathrm{GHz}$ ) that propagate along the surface of the piezoelectric layer. Meanwhile it presents a successions of resonances each $8 \mathrm{MHz}$, that corresponds to bulk acoustic waves that propagate along the perpendicular direction of the sample forcing the Si beneath the piezoelectric layer to vibrate.

The second part of this hybrid device is formed by the ferromagnetic nanostripes and its contact pads. The crystalline structure of the Ni nanostripes is presented in previous chapters, and it is important to know it, in order to understand the behaviour of the magnetization of the nanostripes. Ni nanostripes have polycrystalline structure, with different grain sizes $\left(D_{1}=11\right.$ and $\left.D_{2}=18 \mathrm{~nm}\right)$. The AMR values obtained by measurements in the Ni nanostripes are within the range of $1 \%-1.5 \%$. Furthermore, these measurements show that 
the preferential magnetization direction (anisotropy) is misaligned $45^{\circ}$ and $55^{\circ}$ away from the long axis of the nanostripe. The main information about the magnetic structure of the nanostripes are obtained through magnetoresistance (AMR) measurements performed with a magnetic field applied parallel to the long axis of the nanostripe. Two main contributions to the AMR measurements can be observed. The first one depends on the rotation of the magnetization that occurs when sweeping the magnetic field from saturation to low fields. This generates a decrease in the AMR signal, which shows that magnetization is misaligned from the long axis of the nanostripe at remanence $(\mathrm{H}=0)$. Meanwhile, measurements of magnetic force microscopy (MFM) show that at remanence, the Ni nanostripes form a magnetic structure of alternated oriented magnetic domains. Therefore, it is concluded that the nanostripes at low fields present a multi-domain structure, whose magnetization is oriented away from the long axis of the nanostripe. This magnetic structure is also obtained by the micromagnetic simulations performed the Dpto of Fisica Aplicada of University of Salamanca, where y-oriented magnetic domains can be observed separated by x-oriented domain walls (DW's). Minor loop measurements show that the dynamic process from saturation to the multi-domain structure is perfomed by rotation, due to the reversibility presented in the minor loops, whereas irreversibility observed in the minor loops are related to nonreversible transitions associated to the emergence of a domain whose magnetization points in the opposite direction to the initial saturating direction

Current pulse injection experiments are performed in the Ni nanostripes. These experiments are carried out by saturating the magnetization in one direction and then, taking the external magnetic field to a low field of opposite direction, called injection field $H_{i n j}$. At this point a controlled single current pulse is passed through one of the current lines that connect the nanostripe. By this, we can generate a large magnetic field of a controlled amplitude and time span in order to initiate the reversal process. Experiments are performed where several hundreds of single current pulses were injected in the nanostripe in order observe the triggering of non-reversible events. By the measurement of the resistance $100 \mathrm{~ms}$ before $\left(R_{1}\right)$ and $100 \mathrm{~ms}$ after $\left(R_{2}\right)$ the current pulse application, we are able to determine the triggering of non-reversible events. Results presented in the nanostripe with a grain size of $D_{1}=11 \mathrm{~nm}$ show that at very low injection fields, non-reversible events can be triggered, however $100 \%$ effiency is not found with any parameters of the current pulses. The threshold to count as 
positive a non-reversible event is chosen as the resistance drop due to the nucleation of a $\mathrm{DW}$ in $\mathrm{Ni}(\Delta R=0.05 \Omega)$. The second nanostripe with a grain size of $D_{2}=18 \mathrm{~nm}$ present even fewer events that can be counted as positive $(\Delta R>0.5 \Omega)$ and the histograms show a larger dispersion as a function of $\Delta R$ without any discontinuity. This could be related to the gradually triggering of the non-reversible events that in this nanostripe can be observed in figure 61. 


\section{EFFECTS OF SAW'S IN MAGNETOSTRICTIVE NANOSTRIPES}

The effects that generate the application of surface acoustic waves (SAW's) have been studied, up to this day, in several magnetic nanostructures. However, in this thesis we report to our knowledge, the first experiments of application of SAW's in magnetic nanostripes. The expected effects of the application of the SAW'S are reported in previous works carried out on this topic. The SAW's have been proven to induce an additional anisotropy in Co nanorectangles [Davis, 2010] and in Ni nanosquares [Förster, 2016] in the direction of the propagation of the waves. SAW's have also been shown to promote the switching in ferromagnetic thin films [Tejada, 2017] and elliptical Co nano-elements [Sampath, 2016]. Another effect reported of the application of the SAW's is the reduction of coercive fields in thin films of galfenol [Li, 2014] and (Ga,Mn)(As,P) [Thevenard, 2016]. Therefore, it is worth to study the possibility of the SAW's enhancing the DW nucleation, since [Parkes, 2012] showed experimentally that in an epitaxial-deposited Galfenol film, an irreversible $90^{\circ}$ switching of the magnetization, mediated by DW movement, can be achieved in absence of any external magnetic field. We have also studied the enhacement of the DW dynamics in the nanostripes due to the presence of SAW's.

In this chapter the magnetization of magnetic nanostripes is probed, through magnetoresistance (AMR) measurements, under the application of SAW's. We perform complete AMR loops in order to observe the decrease in coercivity and also minor loops in case the SAW's assist the DW nucleation. Furthermore, current pulse injection experiments are performed when a non-reversible event has occured in the nanostripe in order to observe differences in the DW dynamics in absence and in presence of SAW's. 


\section{A. Characteristics of the Applied SAW's}

The power of the RF signal applied to the SAW resonator is set in $\mathrm{dBm}$ by the signal generator, which can be turned into $\mathrm{mW}$ by the equation :

$$
P_{m W}=1 m W \cdot 10^{P_{d B m} / 10}
$$

The power that carries the wave emitted by the resonator depends on the quality of its IDT. The efficiency in the emission is measured by the $S_{11}$ parameter, which usually shows that our devices emit $>90 \%$ of the power applied by the signal generator. In order to calculate the amplitude of the travelling SAW's, we can use the formula for the average power transmitted in 1D travelling waves, given by:

$$
\frac{k_{e}^{2}<P>}{S}=\frac{1}{2} \cdot v \cdot\left(\xi_{0}^{2} \cdot \omega^{2}\right) \cdot \rho
$$

where $\langle P\rangle$ is the power applied by the IDT, $S$ is the surface of the wavefront, where the power is distributed, $v$ is the sound velocity of the wave in this medium $(v=\lambda \cdot f=3360$ $\mathrm{m} / \mathrm{s}$ ), which is very close to the values reported by [Tang, 2016], $\omega$ is the angular frequency of the wave $\left(\omega=2 \pi \cdot 1.2 \cdot 10^{9} \mathrm{~s}^{-1}\right), \rho=3500 \mathrm{~kg} / \mathrm{m}^{3}$ is the density of the material and $\xi_{0}$ is the amplitude of the SAW's.

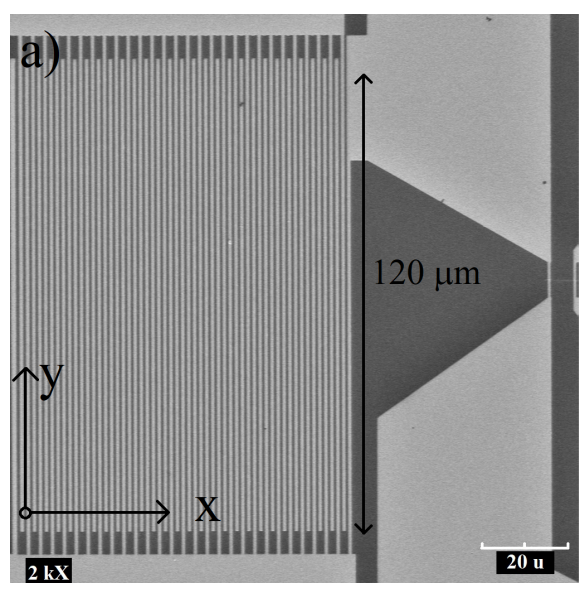

b)

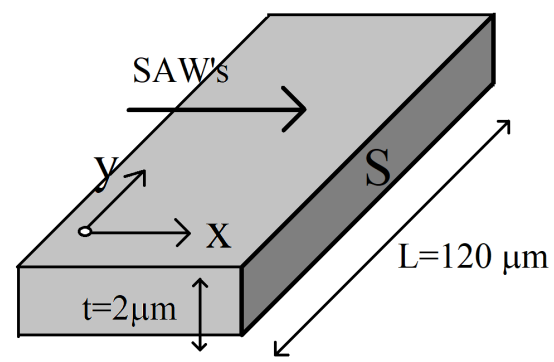

FIG. 70: a) SEM image of the IDT of the SAW resonator in the straintronics device. b) Surface area of the wavefront $(S)$ given the lenght of the IDT and the thickness of the piezoelectric layer. 
The effective electromechanical coupling factor is taken from [Tang, 2016] as $k_{e}=5 \%$. The surface of the wavefront is calculated by multiplying the length of the IDT and the thickness of the piezoelectric layer $\left(S=2.4 \cdot 10^{-10} \mathrm{~m}^{2}\right)$, this is displayed in figure 70 . In table IV, we present $P_{d B m}$, the power in $\mathrm{dBm}$ set to the signal generator, $P_{m W}$ the power in $\mathrm{mW}$ and the amplitude of the SAW's $\left(\xi_{0}\right)$ calculated by equation (19) is in the range of nanometers.

\begin{tabular}{||ccc||}
\hline$P_{d B m}(d B m)$ & $P_{m W}(m W)$ & $\xi_{0}(\mathrm{~nm})$ \\
\hline \hline 19 & 79 & 0.18 \\
18 & 63 & 0.16 \\
17 & 50 & 0.14 \\
16 & 40 & 0.13 \\
15 & 31 & 0.11 \\
10 & 10 & 0.06 \\
5 & 3 & 0.03 \\
0 & 1 & 0.02 \\
\hline
\end{tabular}

TABLE IV: In this table it is displayed the power applied to the SAW resonator in $\mathrm{dBm}$, the adjusted power of the emitted SAW's in $\mathrm{mW}$ and the amplitude of the SAW's in $\mathrm{nm}$.

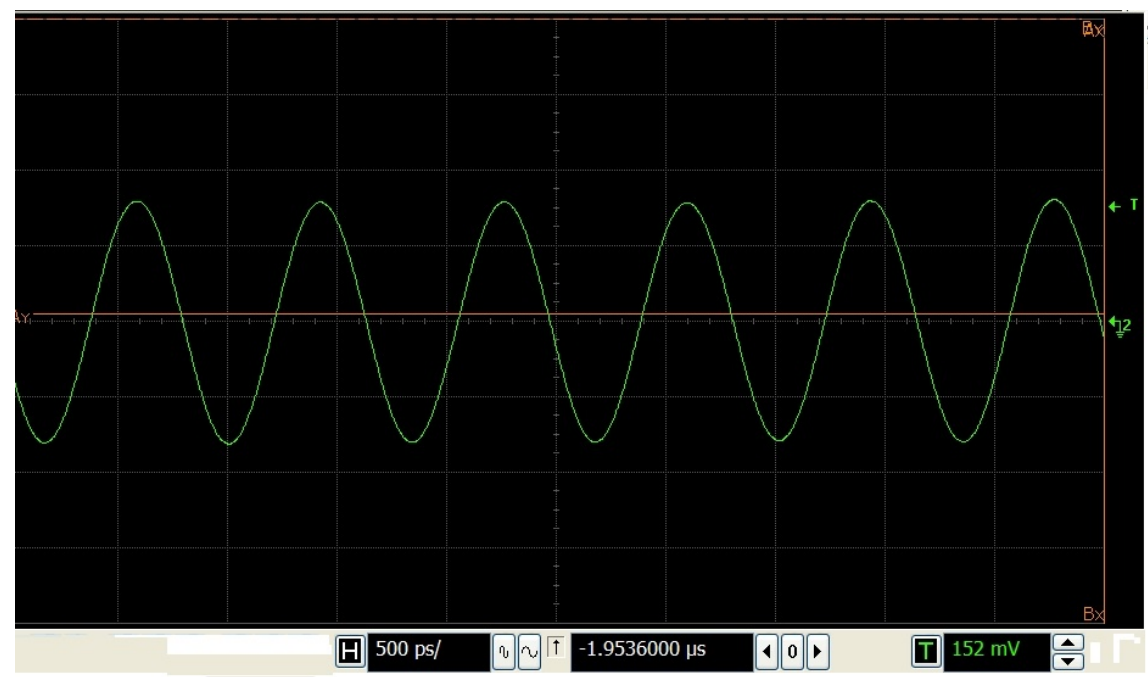

FIG. 71: Screen capture of the RF signal probed from the SAW resonator at a power of $+19 \mathrm{dBm}$. 


\section{B. Effects of SAW's in Magnetoresistance of Ni Nanostripes}

In this section we describe the effects of the application of the SAW's in the magnetoresistance measurements of the Ni nanostripes. The relative geometry between the SAW's and the nanostripe is depicted in figure 72 , where the measurement setup is drawn over a SEM image of the nanostripe.

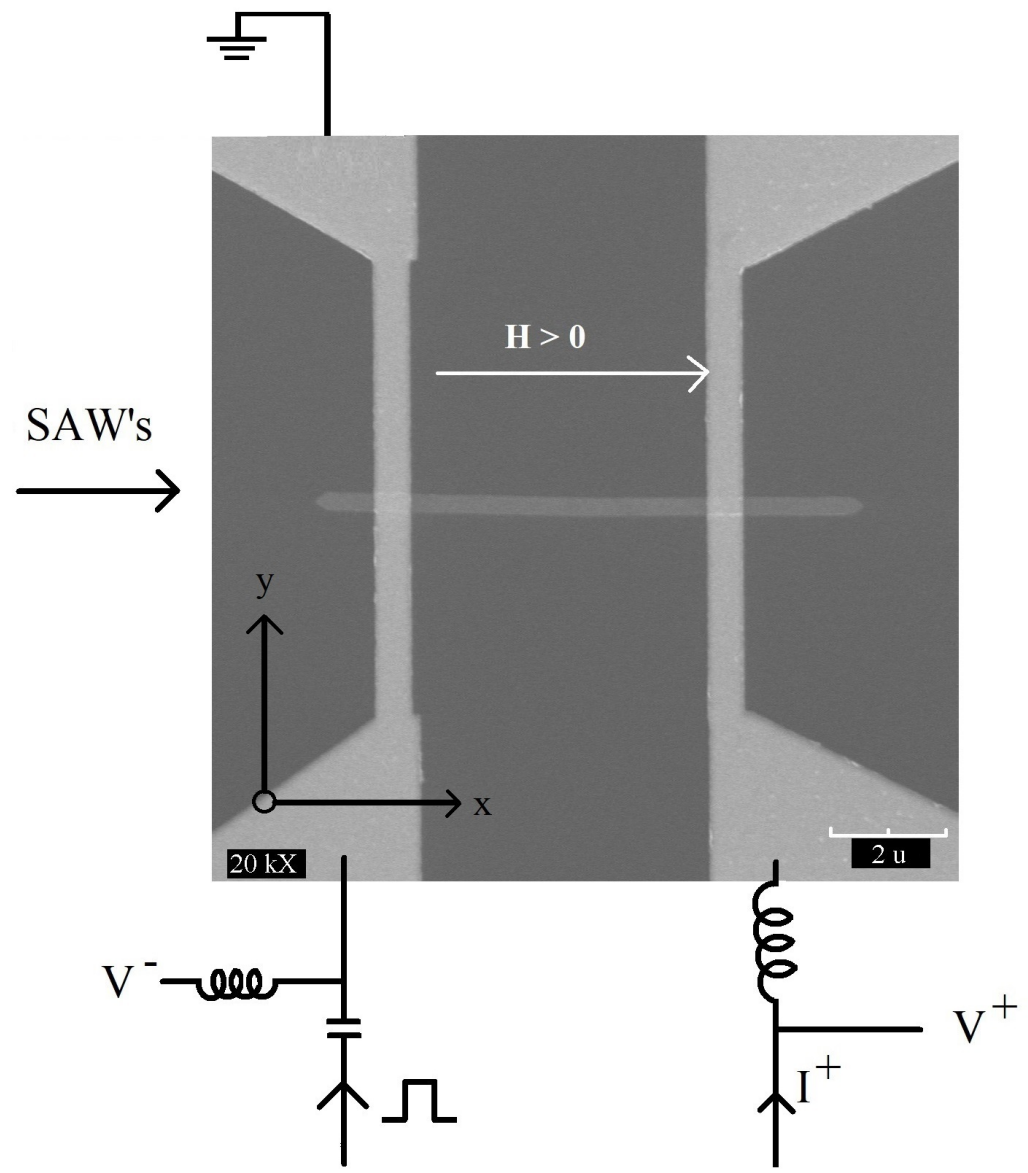

FIG. 72: Schematics of the experimental set up for magnetoresistance measurement and pusle injection drawn on top of a SEM image of the Ni nanostripe. Relative directions of SAW's propagation and external magnetic field are depicted.

Magnetoresistance measurements (AMR) are performed by forcing a DC current $\left(I^{+}=\right.$ $100 \mu \mathrm{A})$ to flow along the nanostripe and probing the voltage in the contact pads $\left(V^{+}-V^{-}\right)$ as a function of an external magnetic field $(H)$. An electrical signal is applied by the signal generator (Keysight model N5181B) to the SAW resonator. Once the signal generator is 
on, we perform AMR measurements. The first visible effect of the application of the SAW's towards the nanostripe is a jump in the resistance. This jump is added as a voltage offset to the AMR curve, and its magnitude depends on the power of the signal applied. Figure 73 shows the AMR curves in presence of SAW's for different signal powers. This resistance offset has two contributions; the acoustoelectric effect and the increase in temperature $(\Delta T)$ that generates the SAW's along their propagation direction. An estimation of $\Delta T$ is calculated in Chapter VIII. section D, whereas the acoustoelectric voltage is studied in Chapter IX. section A.

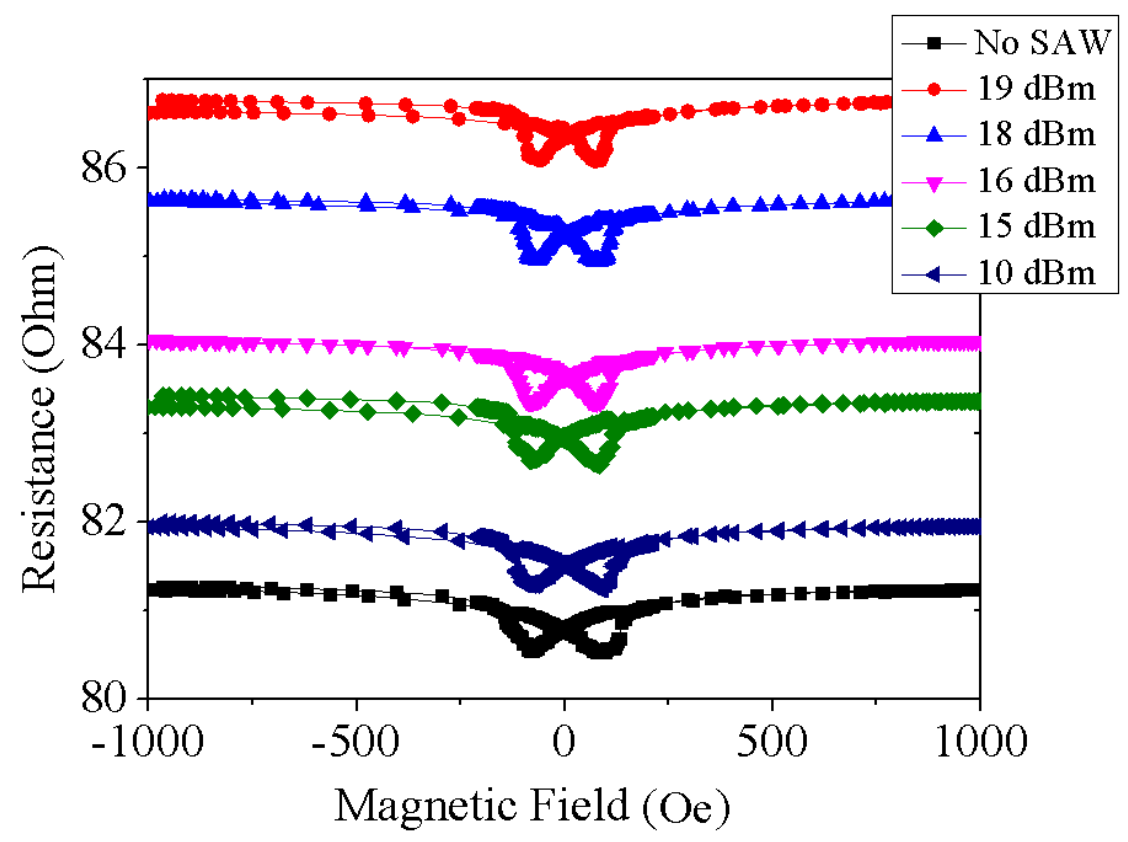

FIG. 73: Magnetoresistance curves for a Ni nanostripe in abscense of SAW's (black curve) and in presence of SAW's with different amplitudes applied. The jump in resistance is dependent on the power of the signal applied.

Magnetoresistance curves present characteristic changes due to the application of the SAW's that can be related to changes in the magnetization state of the nanostripes. Results are presented in figure 74, where AMR curves in absence (black curves) and in presence of SAW's (red curves) are compared. In this case, the signal generator power is set to 19 $\mathrm{dBm}$. For the sake of clarity the curves are displayed in terms of their percentage change in resistance. We present the measurements performed for the set of nanostripes whose grain size is $D_{2}=18 \mathrm{~nm}$ with an anisotropy direction 55 degrees away from the long axis of the 
nanostripe. It can be observed that the slope of the AMR signal from saturiation to low fields is smaller in presence of SAW's. As the SAW's propagation direction is parallel to the DC current flow, we can conclude that SAW's force the magnetization in the direction of the wave propagation. The application of SAW's also decreases the coercive field. In figure 74 it is shown a coercivity reduction of $17 \%$. Another feature the AMR curve presents when SAW's are applied is an asymmetry with respect to the external magnetic field applied, whereas in absence of SAW the AMR curve is symmetric respect to the $H=0$ line (this is discussed in Chapter IX, section B.).

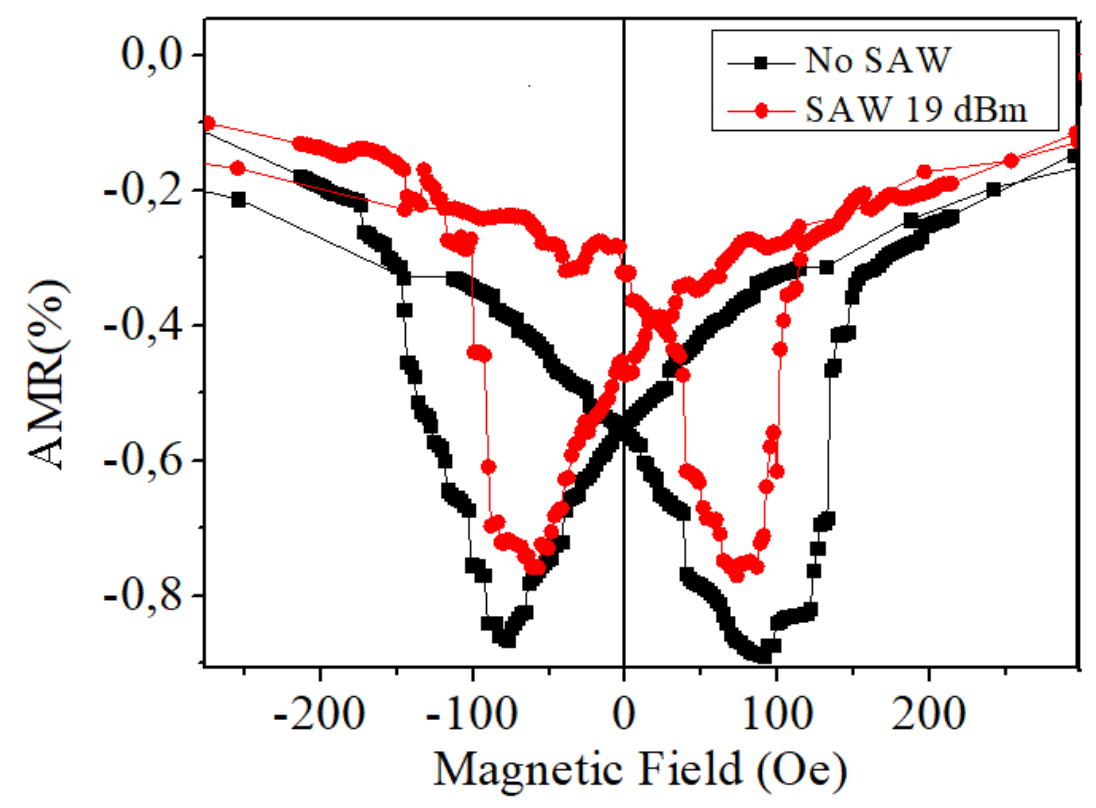

FIG. 74: Comparison of MR measurements in a nanostripes, without (black curve) and with (red curve) applied SAW's with a amplitude of $19 \mathrm{dBm}$ for nanostripes of grain size $D_{2}$. It can be observed how the SAW's decrease the coercive fields.

The effects of the SAW's in the magnetoresistance measurements depend on the power of the signal applied to the SAW resonator. As the power increases, all of these effects are enhanced. At low enough power applied, these effects fade away and below a power of 10 $\mathrm{dBm}$, the effects of the SAW's in the MR curves are not noticeable. 
The fact that the coercive field decreases indicates that the SAW can either promote non-reversible transitions or assist the motion of the DW's along the nanostripe (or both). Magnetoresistance complete loops do not allow to identify the moment at which the magnetoresistance becomes irreversible. Thus, it is necessary to measure minor loops in order to identify at which point the loops turn hysteretic, what allows us to infer that a non-reversible event has occured. Minor loops measurements are performed in absence and in presence of SAW's with a signal power applied of $+19 \mathrm{dBm}$. Both measurements are compared and the results are displayed in figure 75 , where the magnetoresistance is measured from negative saturation down to -4 Oe.

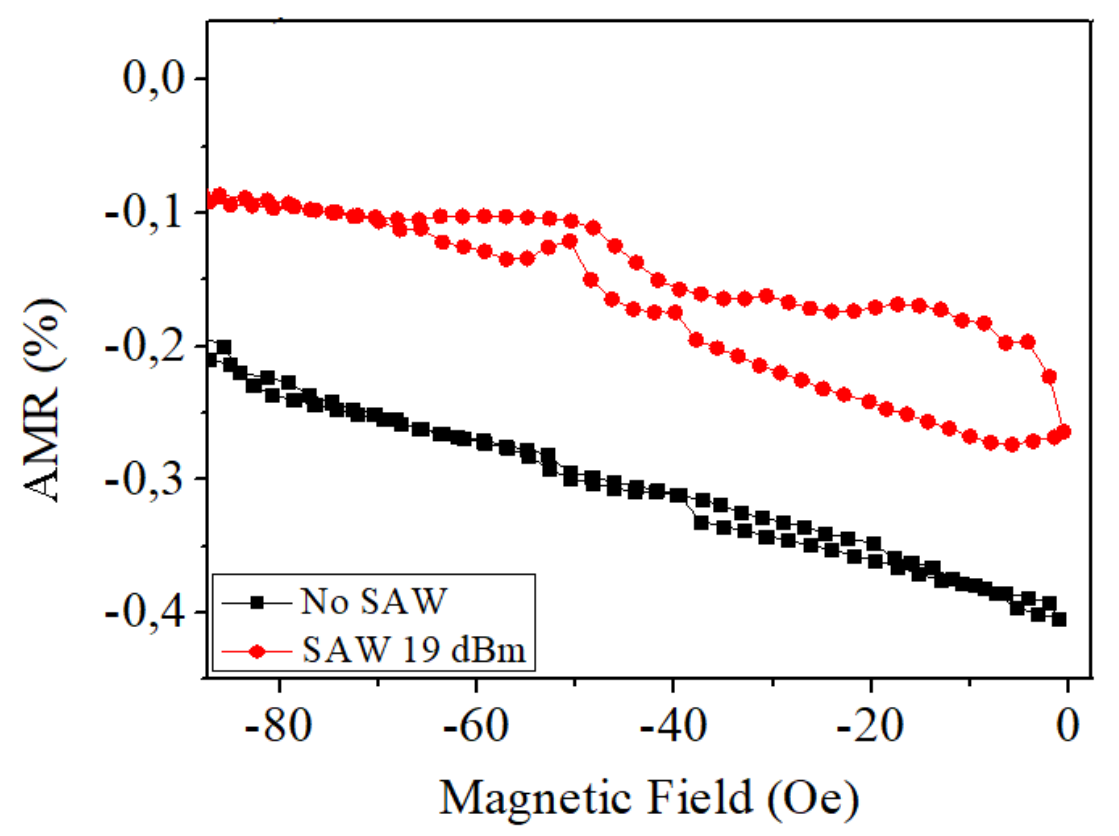

FIG. 75: Minor loops measured from negative saturation down to -4 Oe with SAW's applied at $19 \mathrm{dBm}$ (red curve) and in absence of SAW's (black curve) for nanostripes with grain size $D_{2}$. Hysteretic behaviour is observed when the SAW's are applied despite the magnetic field does not switch its direction.

In figure 75, we show two minor loops, without SAW (black) and with $+19 \mathrm{dBm}$ SAW (red). Results show that hysteresis appears before when SAW's are applied to the nanostripe. The SAW leads to a non-reversible minor loop even if the field does not switch its direction. This can be achieved only for a power larger than $+15 \mathrm{dBm}$ and it happens in all the 
nanostripes measured, even in nanostripes deposited in different conditions with slightly smaller average grain size $(11 \mathrm{~nm})$. This irreversible minor loop indicates that the SAW can assist the nucleation and the movement of magnetic domain walls, even before switching the direction of the external field.

\section{Micromagnetic simulations}

Micromagnetic simulations were performed using the Mumax program [Vansteenkiste, 2017] by the Departament of Fisica Aplicada of University of Salamanca on a nanostripe with dimensions of $35 \mathrm{~nm} \times 10 \mu \mathrm{m} \times 400 \mathrm{~nm}$ and magnetic parameters of exchange coupling $A=1.05 \cdot 10^{-11} \mathrm{~J} / \mathrm{m}$, saturation magnetization $M_{s}=2.38 \cdot 10^{5} \mathrm{~A} / \mathrm{m}$ and uniaxial anisotropy $K=6.0 \cdot 10^{3} \mathrm{~J} / \mathrm{m}^{3}$. The polycrystalline grain structure is modelled via a Voronoi tessellation of the system in polygonal regions of average diameter of $D=20 \mathrm{~nm}$. The external field was ramped in steps of $1 \mathrm{mT}$, maintaining each field for a time interval $t=100 \mathrm{~ns}$ and with $\alpha=0.01$ as damping constant. In the simulations presented in figure 76 a), SAW's of different amplitudes are applied to the nanostripe and the magnetization is displayed as a function of the external magnetic field. As it can be observed in figure 76 a) the simulations reproduce most of the features observed in the experimental data. Most significantly, SAW's promote a reduction of the coercivity. Also, the simulations reproduce that the slope of the initial reversible part of the loop, when the field is decreased from saturation, is smaller in presence of SAW's. Finally, the approach to saturation is more abrupt once the coercive field is surpassed, with fewer but more pronounced jumps. The simulations are compared with the experimental measurements of the magnetoresistance in presence of SAW's for different signal powers (figure 76 b) ). The pressure carried by the SAW's experimentally, is computed from the amplitudes presented in table IV (Chapter VIII. section A) by the Hook's law (20), where $Y$ is the Young modulus of the ScAlN (180 GPa) [Teshigahara, 2012], $k$ is the wavenumber of the SAW's $\left(k=\frac{2 \pi}{2.8 \mu m}\right), \xi$ is the expression for the acoustic wave and $\xi_{0}$ is the amplitude of the wave.

$$
p=Y \cdot \frac{\partial \xi}{\partial x} \rightarrow|p|=Y \cdot k \cdot \xi_{0}
$$

All the features observed in the simulations (figure $76 \mathrm{c}$ )) can be understood considering that, to a first approximation, the effect of SAW's is equivalent to a field along the long 
a)

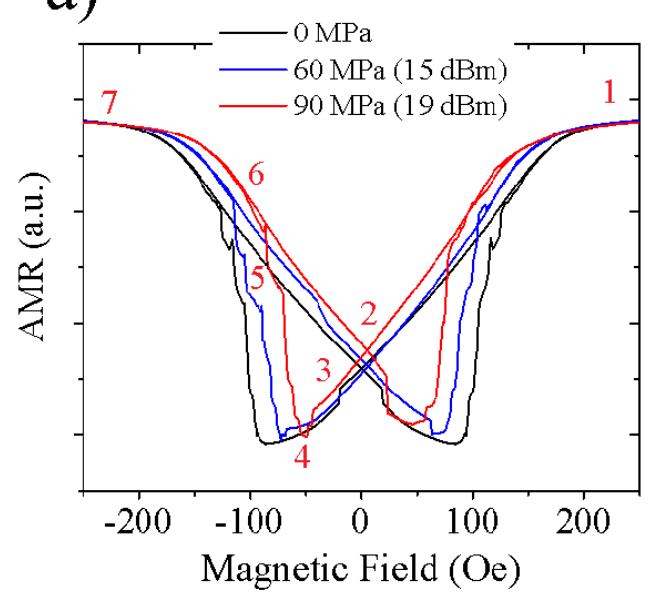

b)

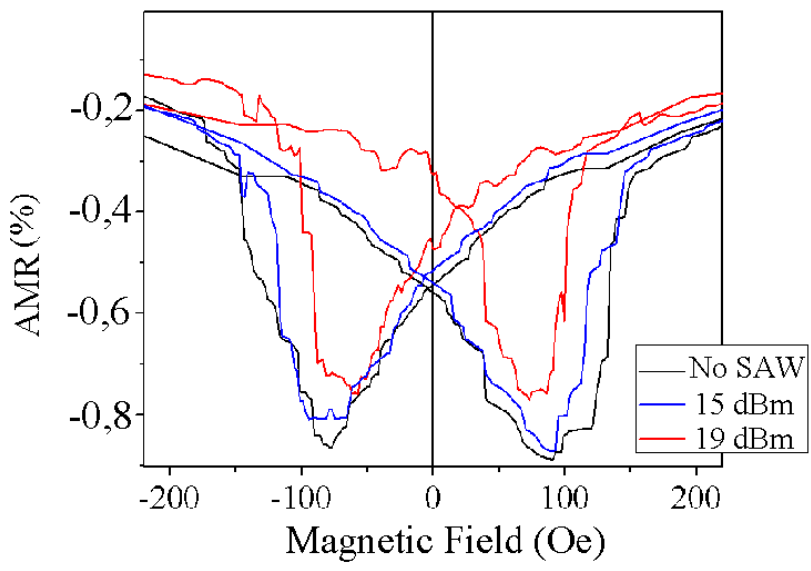

$90 \mathrm{MPa}(+19 \mathrm{dBm})$

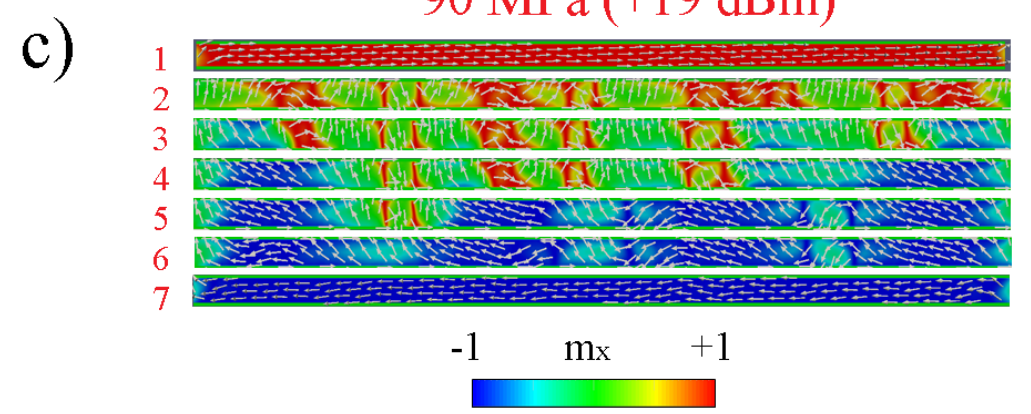

FIG. 76: a) Simulated AMR for different SAW's amplitude defined by the pressure applied to the Ni nanostripe. As the amplitude of the SAW's increase, the coercive field on the nanostripe decreases. b) Experimental AMR measurements for different powers applied to the SAW resonator. c) Snapshots of the magnetization process at different fields with 90 MPa amplitude SAW.

axis direction (x direction), thus favoring longitudinal orientation of the spins. As the field is reduced from saturation and the system breaks into domains oriented along the direction perpendicular to the long axis (y direction), the walls separating them, magnetized along the positive $\mathrm{x}$ direction, are wider with SAW and, when two of them are close together, they tend to merge into a single extended region predominantly oriented along the $\mathrm{x}$ direction. Consequently, fewer y-oriented domains are present in the system with wider x-oriented walls separating them, which leads to a smaller slope in the initial reversible part in the loop and a higher remanence. Also, since there are less domains, irreversible jumps are scarce but more pronounced. In particular, around the coercive field a few large irreversible jumps are observed corresponding to long domain wall displacements and subsequent annihilation. 
SAW's also introduce a periodic excitation in the DW's that favors depinning, which explains the reduction in the coercivity. Finally, once all the irreversible jumps have taken place, SAW also facilitates the alignment of the magnetization with the longitudinal field, which gives a more abrupt approach to saturation.

a)

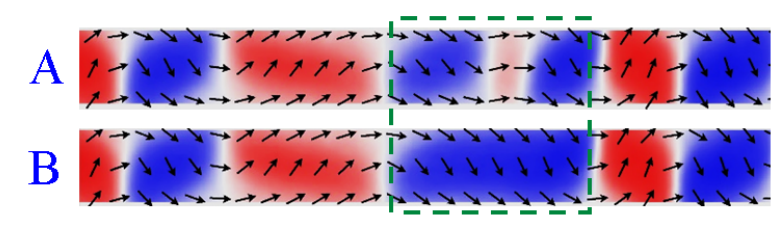

b)

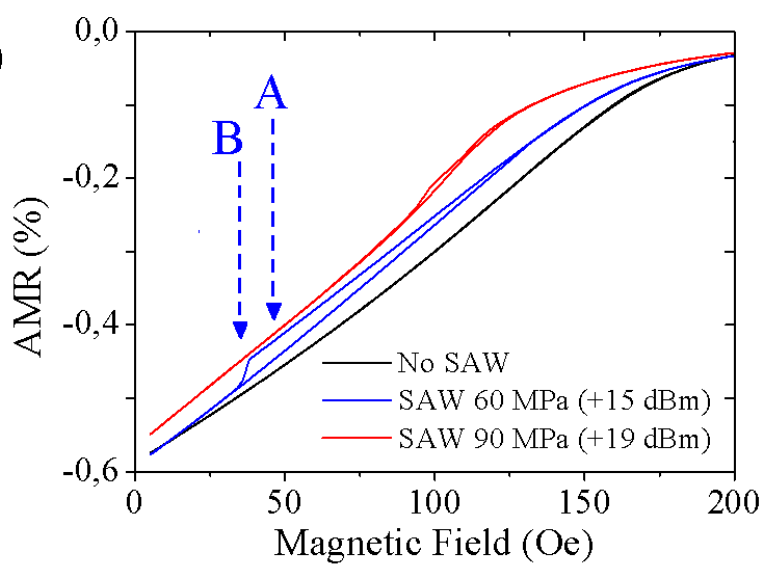

FIG. 77: a) Snapshots of micromagnetic simulations that show the magnetic configuration of a section of the nanostrip before (A) and after (B) the hysteretic jump. b) Example where the simulation could reproduce the experimental hysteretic minor loop of figure 75 . The minor loops run from positive saturation to a positive small field

Simulations are performed with the objective of reproducing the irreversible jumps that occur before the switching of the external magnetic field (figure 75). This is achieved, as shown in figure 77. However, specific simulations conditions are necessary to reproduce this behaviour. In the simulation presented figure 77, the external field was ramped in steps of 2 Oe, maintaining each field for a time interval $t=4 \mathrm{~ns}$ and with $\alpha=1$ as damping constant. The snapshots of a section of the nanostripe (figure 77 a)) show how, under these particular conditions, the SAW is able to make two DW's collapse and disappear (marked with a dashed square), generating the jump in the AMR value. Therefore, the experimental finding shown in figure 75 , seems to be a consequence of the SAW affecting metastable magnetic configurations achieved in the nanostripe as the field is ramped 


\section{DW Dynamics Assisted by the SAW's}

We have shown the enhancement of the non-reversible changes in the magnetization due to the SAW's, but we aim to deepen into the effects that the SAW's generate in the movement and depinning of the DW's. In this section it is reported the effect of current pulses of local field on the nanostripes in presence and in absence of SAW's. The schematics of this experiment is presented in figure 78 .

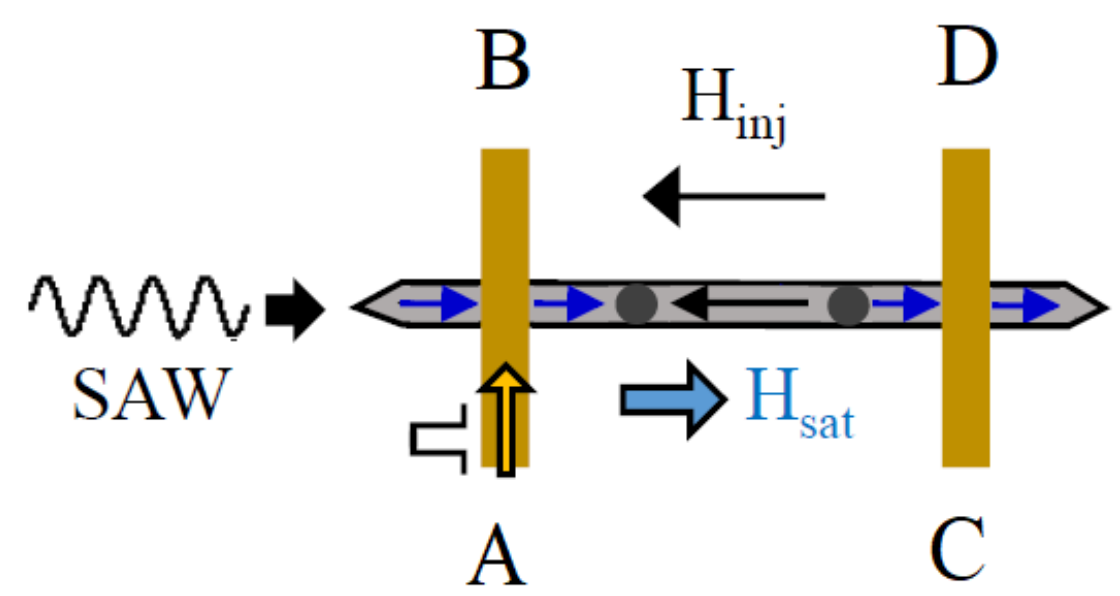

FIG. 78: Schematics of the injection of a current pulse. Saturation and injection field directions are indicated as well as the propagation direction of the SAW's.

The experiment is performed as follows: first we apply a saturating magnetic field in one direction $\left(H_{\text {sat }}\right)$. After that, the magnetic field is taken to a low value in the opposite direction $\left(H_{i n j}\right)$, where the reversal process has already started. At this point, the resistance of the nanostripe is measured $\left(R_{1}\right)$, and a current pulse of local field is injected from contact A to contact B (see figure 78). The Oersted field generated by the current pulse provides a controlled push to the DW's and triggers a sudden irreversible jump of the AMR in the nanostripe $\left(R_{2}\right)$. The amplitude of $\Delta R=R_{1}-R_{2}$ gives an idea of how large is the distance freed by the DW's between two pinning sites. Finally, with the objective to make some statistics, we repeat this sequence hundreds of times for each injection field. This experiment is carried out in presence and in absence of SAW's and the results are compared. 
The current pulses are injected at fields where the reversal process has already become irreversible, therefore, we previously perform minor loops starting from positive saturation, as indicated by the arrows in figure 79. Any minor loop performed in the Ni nanostripe without SAW, would be reversible until reversal fields larger than -30 Oe. This is an indication that, for fields larger than -30 Oe, there are irreversible movements of magnetic domain walls or irreversible rearrangements of the magnetic domains. In figure 79 it is displayed a minor loop measurement from positive saturation down to -45 Oe.

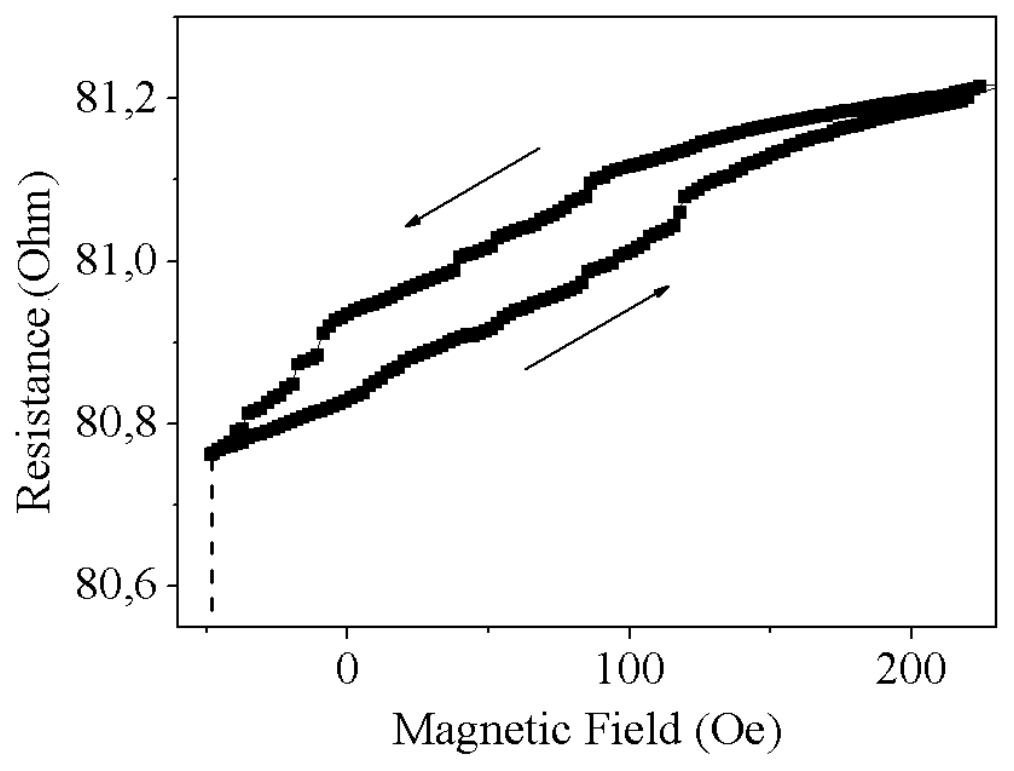

FIG. 79: Minor loop measured between saturation in the positive direction and -45 Oe. Curve shows hysteretic behaviour caused by non-reversible transitions that occur in the magnetization of the nanostripe.

Therefore, we perform this experiment at injection fields larger than -30 Oe $\left(H_{i n j}<-30\right.$ Oe). The current pulses used in this experiment have a duration of $15 \mathrm{~ns}, 4 \mathrm{~V}$ ampliltude and the current density in the current line is $\sim 6 \cdot 10^{7} \mathrm{~A} / \mathrm{cm}^{2}$. The pulses are applied from contact A to contact B, and the local Oersted field generated by the pulse is of the order of 400 Oe just underneath the current line. A screen capture from the oscilloscope of the current pulse of local field applied to the nanostripe while the resonator is on, is presented in figure 80 . 


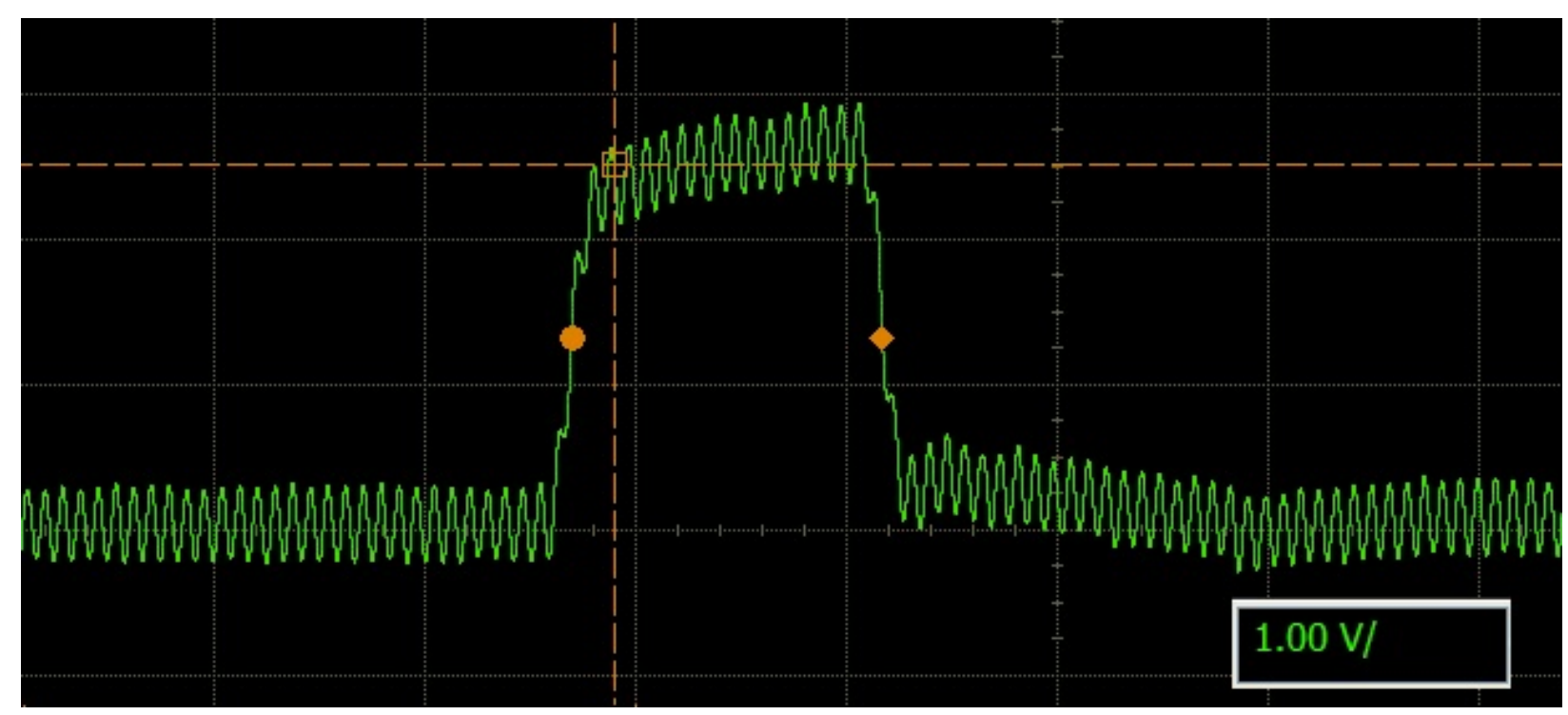

FIG. 80: Screen capture from the oscilloscope of a current pulse applied in presence of the SAW's. The applied pulse is $4 \mathrm{~V}$ of amplitude and $15 \mathrm{~ns}$ of duration. The power of the signal applied to the SAW resonator is $19 \mathrm{dBm}$. Each vertical division is $1 \mathrm{~V}$.

As the injection of a current pulse of local field enhances the reversal process, positive $\Delta R=R_{2}-R_{1}$ are expected, which indicates a final magnetization state more parallel to the long axis of the nanostripe (direction of flow of the DC current) than the initial one $\left(R_{1}<R_{2}\right)$. This process is represented in figure 81 , where the actual magnetoresistance curves under the injection of current pulses are displayed. When the current pulse is injected, the magnetization is taken from a state (1), to another state (2), where state 2 is only reachable at larger fields in absence of the current pulse injection. Simulation snapshots are also displayed, which show the magnetization states 1 and 2 . As it can be seen, the magnetization reversal is performed by the expansion of the blue domains. Therefore, the injection of the current field promotes this expansion which occurs by the movement of the DW's to different pinning sites. The experimental values of the AMR show that $\Delta R$ is larger in presence of SAW's, which is related to a larger expansion of the blue domains due to the movement of the DW's. This makes sense, since the approach to saturation in the AMR curve is more abrupt, and irreversible jumps are scarce but more pronounced when the SAW's are applied, meaning that the SAW's hinder the pinning of the DW's and when the DW's are depinned, they travel larger distances between two pinning sites. 
a)

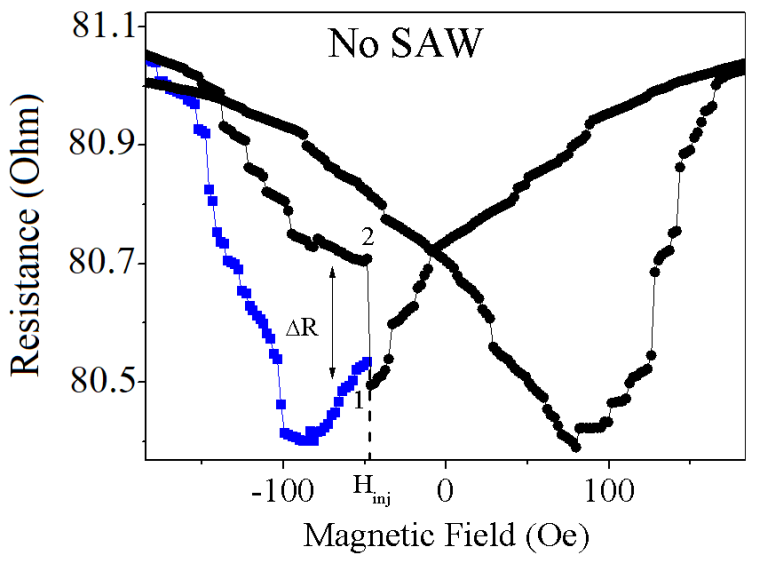

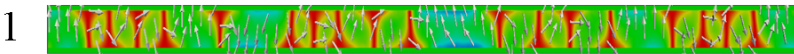

b)

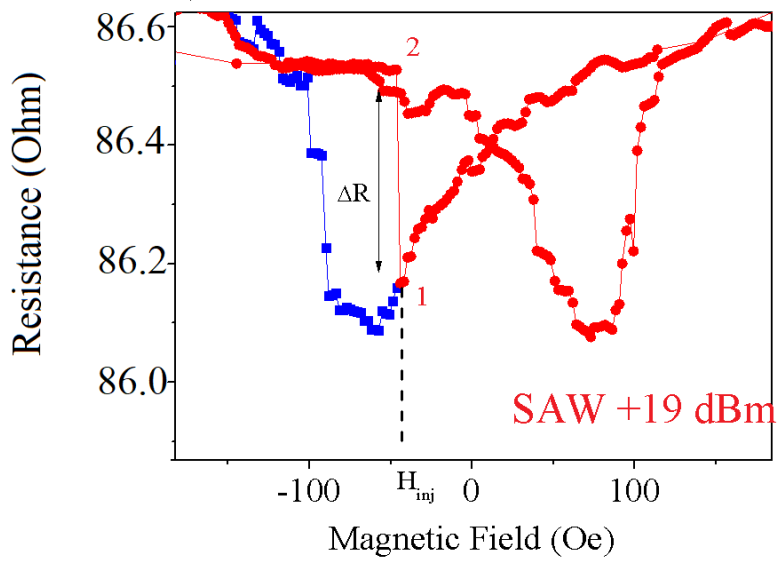

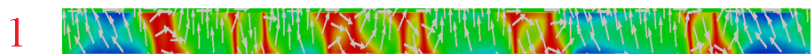

2

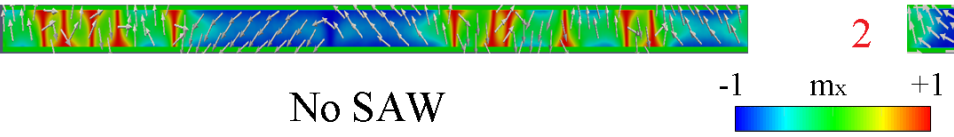

$+1$

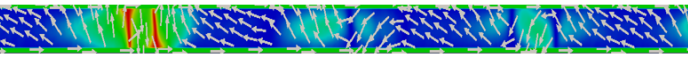

SAW $90 \mathrm{MPa}(+19 \mathrm{dBm})$

FIG. 81: Magnetoresistance measurements where a current pulse is injected at $H_{i n j}=-45 O e$. $\Delta R$ is compared for the case with no SAW's applied a) and with SAW's applied b). Results show a larger $\Delta R$ jump when the SAW's are applied. Blue AMR curves are measurements at same conditions without applying the current pulse at $H_{i n j}$. Snapshots of the magnetization reversal at different states are displayed.

In order to make some statistics this experiment is performed 200 times in presence (red points) and in absence of SAW's (black points) for several injection fields from $H_{i n j}=-30$ Oe to -80 Oe. Figures $82 \mathrm{a}$ ), b) and c) display the number of events as a function of $\Delta R$ at $H_{i n j}=-30,-40$ and $-45 O e$ respectively, where the application of the SAW's shifts $\Delta R$ to larger values. In figure $82 \mathrm{~d}$ ), measurements of $\Delta R$ are presented when the injection field is larger than the coercive field. As it can be seen, when the pulses of local field are applied at $H_{i n j}=-80 O e$ in presence of SAW's, $\Delta R$ has diminished noticeably. This is because the initial state (1), whose resistance value is $R_{1}$ is already close to a saturation state and the effect of the pulse is smaller compared to the effect at lower injection fields. However, at this $H_{i n j}$ in absence of SAW's the resistance value $\left(R_{1}\right)$ of the initial state $(1)$ is at a minimum. Thus, the application of the current pulse generates a large increase in resistance due to the maximum difference in resistance between the initial and the final states . 


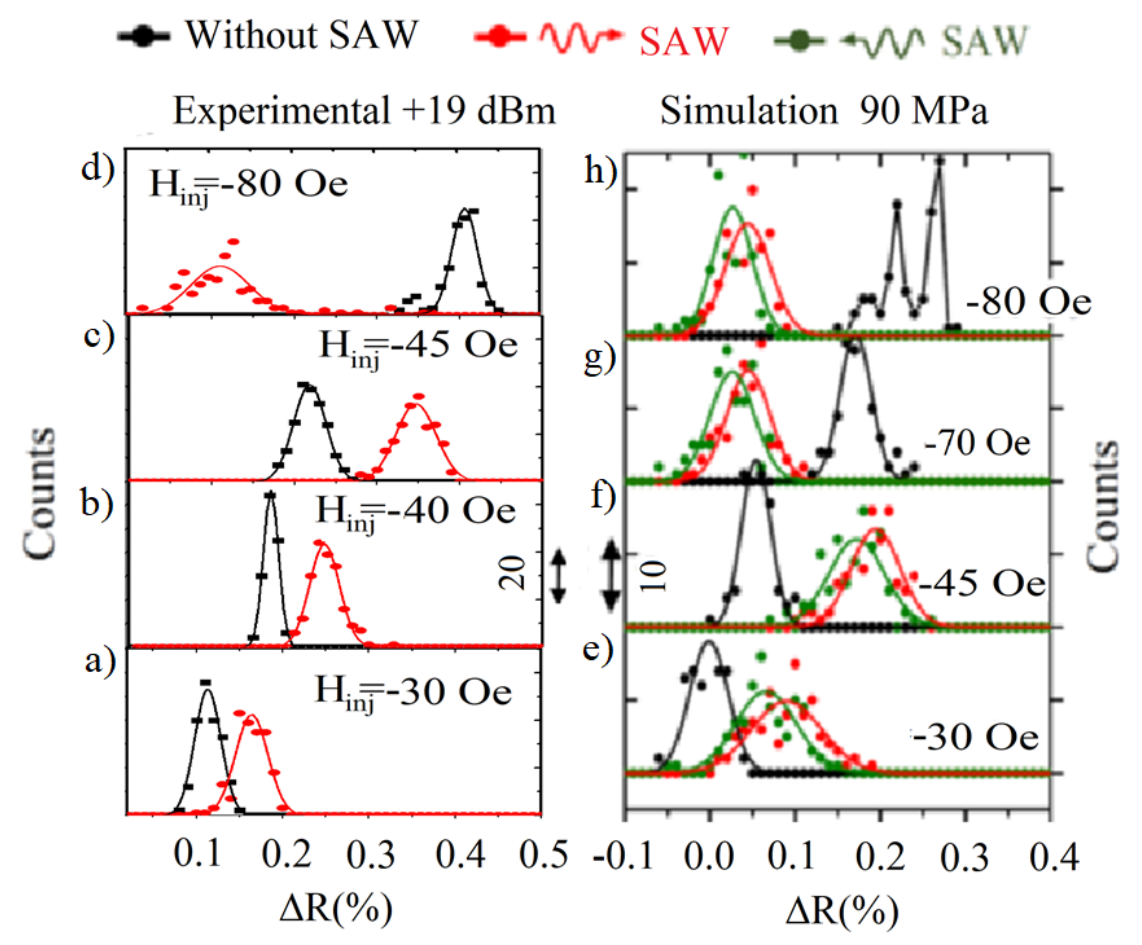

FIG. 82: a)-d) Number of events as a function of the change in resistance $\Delta R$ triggered by the injection of current pulses at different injection fields in presence (red data) and in absence (black data) of SAW's.

Micromagnetic simulations are performed to reproduce the data presented in figure 82 a) - d), following the same sequence as in the experiment. Figure 82 e) - g) shows the main results of these simulations. For each injection field 100 events were computed without SAW (black curves), with $90 \mathrm{MPa}$ SAW travelling in the same direction as in the experiment (red curves) and in the opposite direction (green curves). We considered the same values of $H_{i n j}$ than in the experiment and several features are reproduced. The average $\Delta R$ is larger with SAW, indicating that the SAW induce larger displacements of the DW's. Furthermore, for higher fields the increase $\Delta R$ is smaller with SAW than without SAW, since for those fields the reversal process of the nanostripe is close to saturation. It is interesting the study with the SAW propagating in the opposite direction to the one in the experiment. As it can be observed, a similar statistical distribution is obtained, although slightly shifted towards smaller $\Delta R$ in every case, which means that the direction of travel of the SAW has some effect on the DW displacement. This could be due to transfer of linear momentum between elastic and magnetic subsystems, although this mechanism demands further research. 


\section{Magnetoresitance Measurements on an AC Current}

Up until this point we have presented results of magnetoresistance measured by the application of a controlled DC current through the nanostripe, typically $I=100 \mu A$, which is the current used all along the measurements presented before. In this section we present a series of measurements performed under the application of an AC signal. In this case the resistance is measured by differential signaling. The schematics of the differential measurements setup is depicted in figure 83.

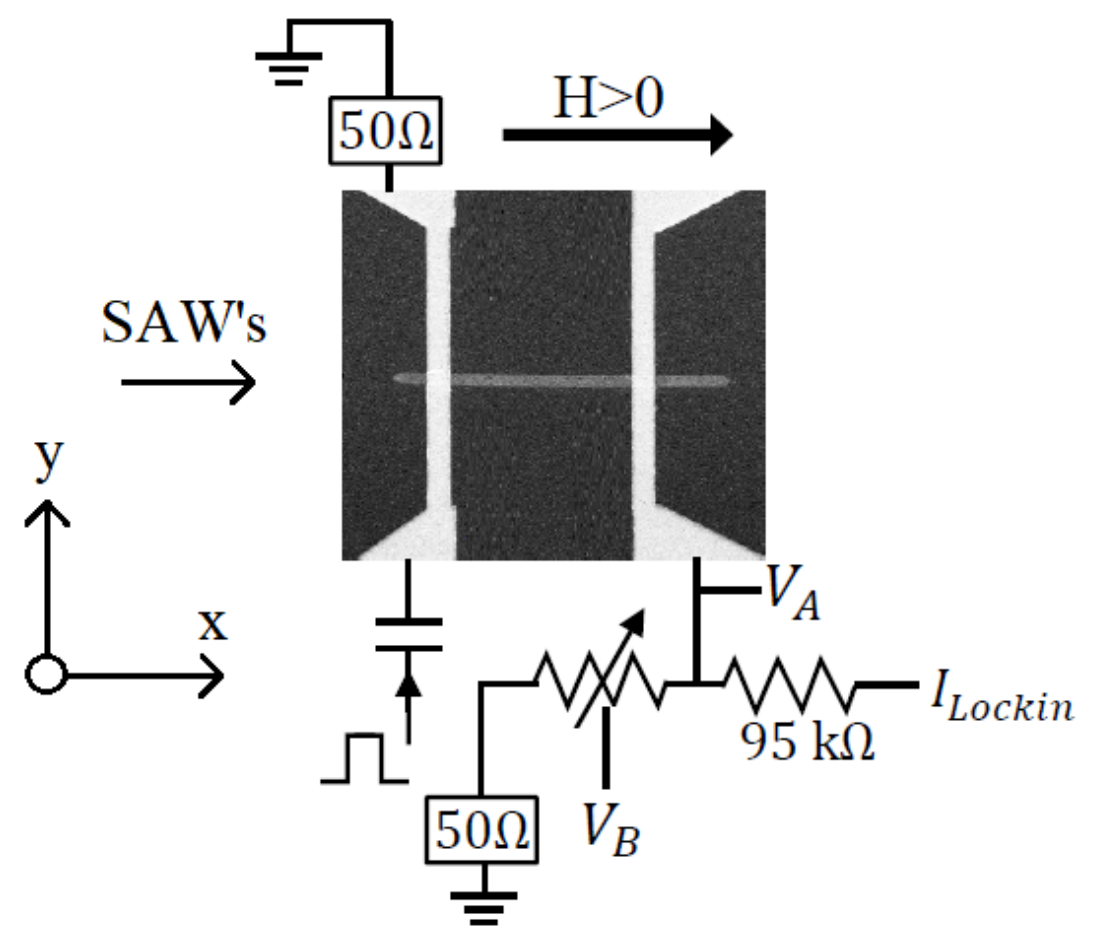

FIG. 83: Experimental setup of AC differential measurements for the magnetoresistance in the Ni nanostripe. A high resistance of $95 k \Omega$ is used to lower the amplitude of the current applied. The potentiometer is used to compensate the voltages measured in $V_{A}$ and $V_{B}$.

In this measurement an $\mathrm{AC}$ input signal from the Lockin amplifier is applied to the nanostripe and the voltages measured in $V_{A}$ and $V_{B}$ are compared. The main advantage of using this method is the diminishing in the electric noise compared to the measurements performed with DC current. The AC input signal applied amplitude is $2 \mathrm{~V}$ at a frequency of $1 \mathrm{kHz}$. In figure 84 it is displayed the comparison of AMR measurements with and without the application of the SAW's at $19 \mathrm{dBm}$. 
a)

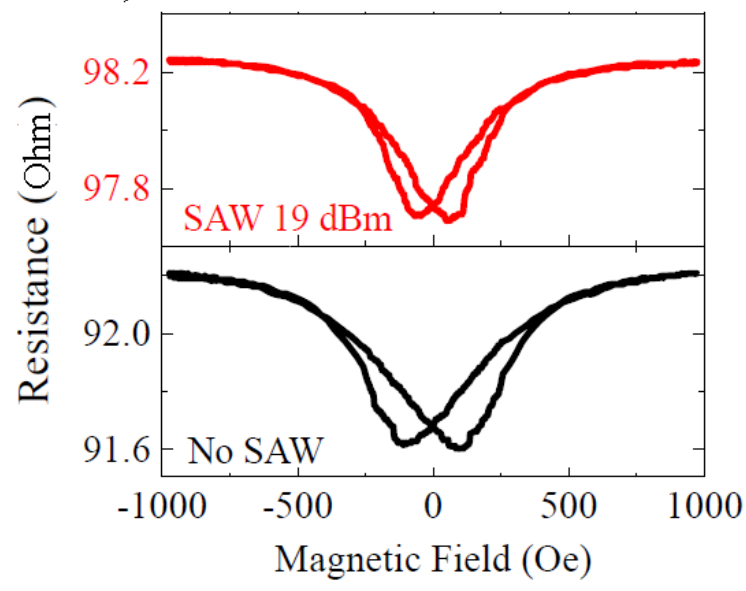

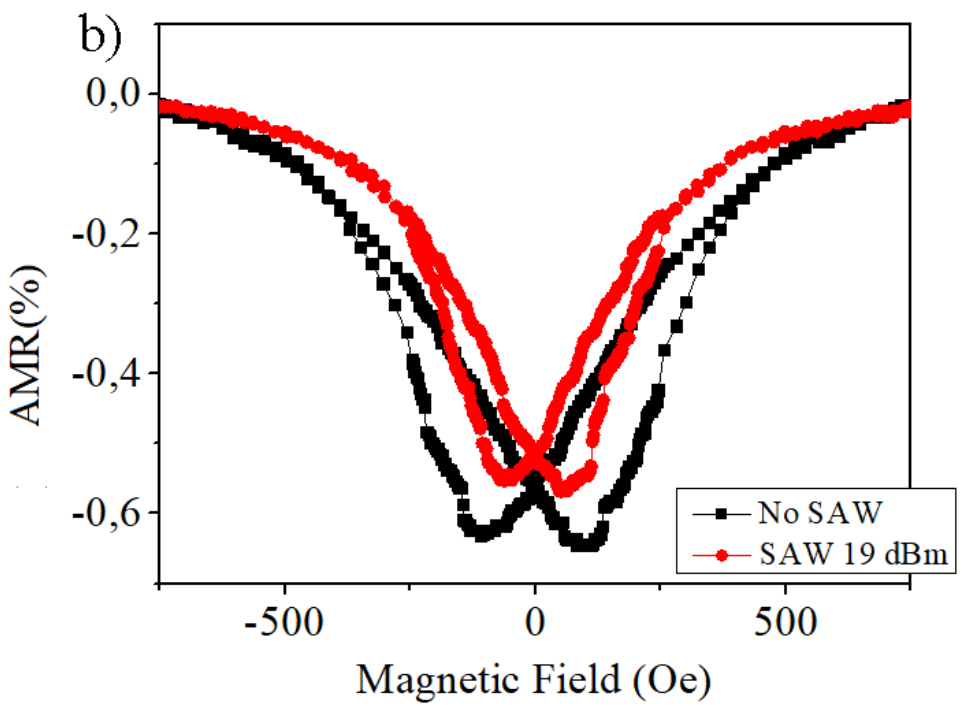

FIG. 84: Comparison of magnetoresistance measurements in a Ni nanostripe without SAW's (black curve) and with SAW's applied to it. Results are presented in terms of the resistance a) and in terms of the change of AMR b).

Magnetoresistance curves are presented in terms of their resistance (figure 84 a)) and in terms of their change of AMR $(84 \mathrm{~b}))$. Results show some of the same effects as before when the DC current was applied along the nanostripe: an increase in resistance, smaller coercive fields and a smaller slope of the signal coming from saturation to low fields. In this measurements the field is ramped one step per second, which is the integration time of the Lock-in, therefore the Barkhausen jumps observed in the DC measurements are averaged. Furthermore, in this case the asymmetry of the AMR curve is not present. The origin of the asymmetry and partially, the increase in the resistance, is the acoustoelectric effect, which is discussed in Chapter IX, section A. As the AC Lock-in resistance measurement filters the DC voltage build up coming from the acoustoelectric effect (see Chapter IX, section A.), we are able to estimate the temperature increase due to the SAW by the change of the electrical resistance in the $\mathrm{Ni}$ nanostripe. The resistance at saturation with no SAW is 92.2 $\Omega$ and with $19 \mathrm{dBm}$ SAW is $98.2 \Omega$. Therefore, if we take the resistivity as a function of the temperature $\rho(T)=\rho_{0}\left[1+\alpha\left(T-T_{0}\right)\right]$ with $T_{0}=300 \mathrm{~K}$ and the temperature coefficient for $\mathrm{Ni}, \alpha=0.006 \mathrm{~K}^{-1}$, we can quickly calculate:

$$
98.2 \Omega=\rho(T) \cdot l / A=\rho_{0}\left[1+\alpha\left(T-T_{0}\right)\right] \cdot l / A
$$




$$
92.2 \Omega=\rho_{0} \cdot l / A
$$

Dividing both equations,

$$
1.065=1+\alpha \Delta T
$$

Therfore $\Delta T=10.9 \mathrm{~K}$

The change in the electric resistance of $\mathrm{Ni}$ indicates that the temperature increase of the nanostripe is not much larger than $10 \mathrm{~K}$ for $+19 \mathrm{dBm}$. This would discard the possibility that the irreversibility of minor loops in presence of SAW'S is induced by heating.

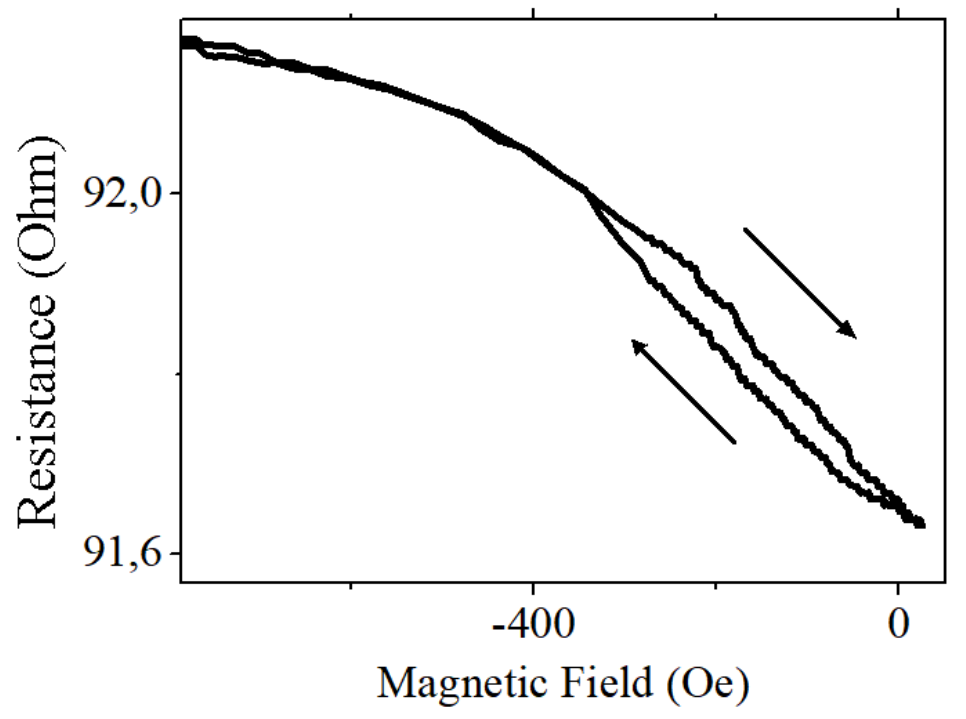

FIG. 85: Minor loops measured in Ni nanostripe with an AC current from negative saturation magnetic field down to 30 Oe. Hysteretic behaviour is presented with a difference of $0.05 \Omega$ between the branches of the loop.

Minor loops are measured under in this setup from negative saturation fields down to 30 Oe. Results are displayed in figure 85. In the minor loop measurement hysteresis is observed. The difference in resistance between the branches of the minor loop is $\Delta R=0.05 \Omega$, a drop in resistance which is caused by a non-reversible transition occured in the nanostripe. Once the presence of the hysteresis at certain fields is confirmed, we performed current pulse injection experiments in order to observe the effect of the SAW's in the DW dynamics. The schematics of the experiment is presented in figure 86, where the relative directions on the 
injection field, saturation field and the current pulses are depicted. We present two different geometries for the injection of the current pulses. The saturation magnetic field is in the negative direction, and the injection field is positive $\left(H_{i n j}=+35 \mathrm{Oe}\right)$, a field large enough as seen in the minor loops to present hysteresis in absence of SAW's. The events are measured 100 times in these two experiments and results are presented in figure 86.

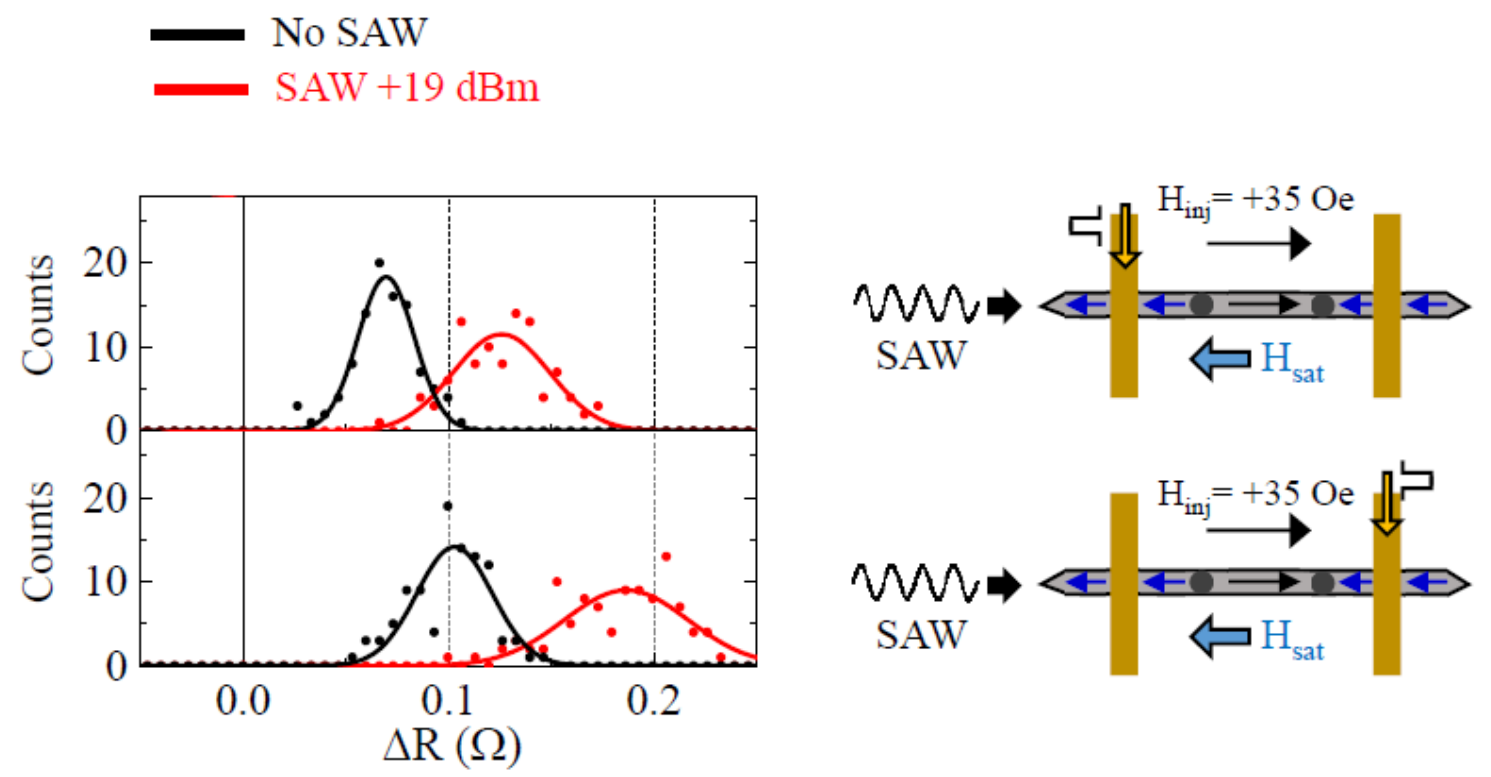

FIG. 86: Changes in resistance due to the application of a current pulse with and without the presence of SAW's. Data results are presented with the most adjusted gaussian curve to the histograms. In the right side the schematics of the geometries of the experiment for each case are depicted.

The results obtained match with the ones from the experiments with DC current. The changes in resistance $\left(\Delta R=R_{1}-R_{2}\right)$ due to the current pulse application are always positive $\left(R_{2}>R_{1}\right)$, because as seen in the minor loop in figure 85 at this injection field $H_{i n j}=+35$ Oe, the DW's are provided with energy to expand, favoring the reversal process. As in the DC experiments, the application of the current pulse in presence of SAW's generates a larger increase of resistance $\Delta R$ than the current pulse application in absence of SAW's, which is evidence of the SAW's providing energy to the DW's. 


\section{E. Effects of SAW's in Magnetoresistance of FeCoB Nanostripes}

We present in this section the magnetoresistance measurements of a FeCoB nanostripe in presence of SAW's. The setup experiment is shown in figure 87, where $\alpha$ is the angle that forms the external magnetic field with the propagation direction of the SAW's.

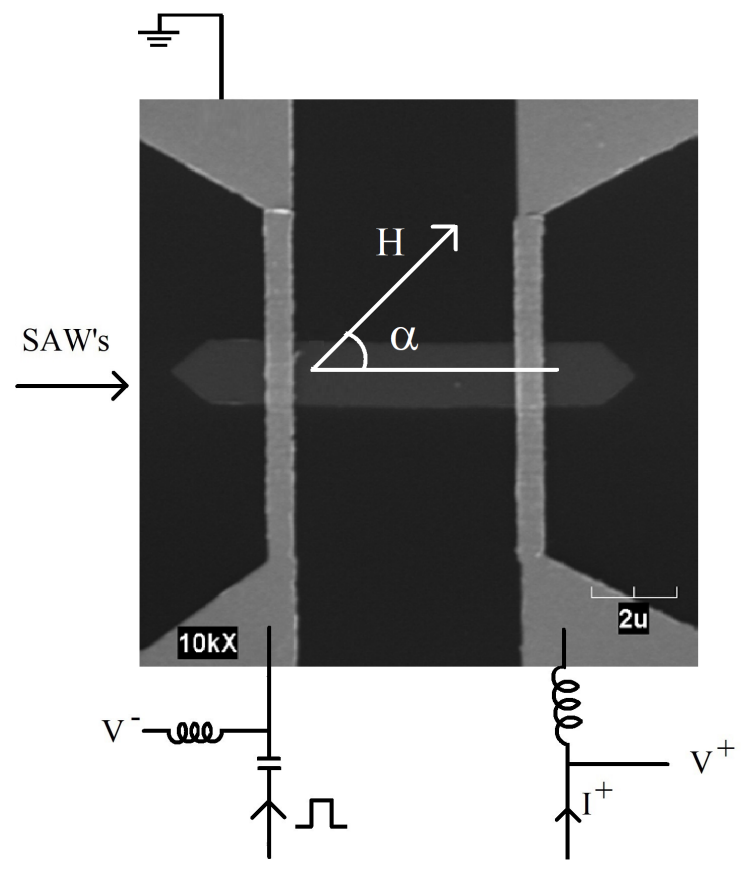

FIG. 87: Experimental setup for a magnetoresistance measurements of a FeCoB nanostripe in presence of SAW's.

In order to understand the results presented in this section it is necessary to take into account the anisotropy direction of this nanostripe. AMR measurements are displayed in figure 88, where magnetoresistance measurements with an external magnetic field applied parallel (black curve) and perpendicular (blue curve) to the long axis of the nanostripe are compared. The AMR measurement performed at $\alpha=0^{\circ}$ show that the resistance signal is almost constant along all the measurement except at \pm 70 Oe where the reversal process takes place. On the other hand, when the measurement is performed at $\alpha=90^{\circ}$ the resistance signal increases progressively from saturation (where the magnetization is perpendicular to the $\mathrm{DC}$ current) to a maximum value at $\mathrm{H}=0$ (where the magnetization is parallel to the $\mathrm{DC}$ current). From these measurements, it becomes clear that the long axis of the nanostripe is the anisotropy direction. 


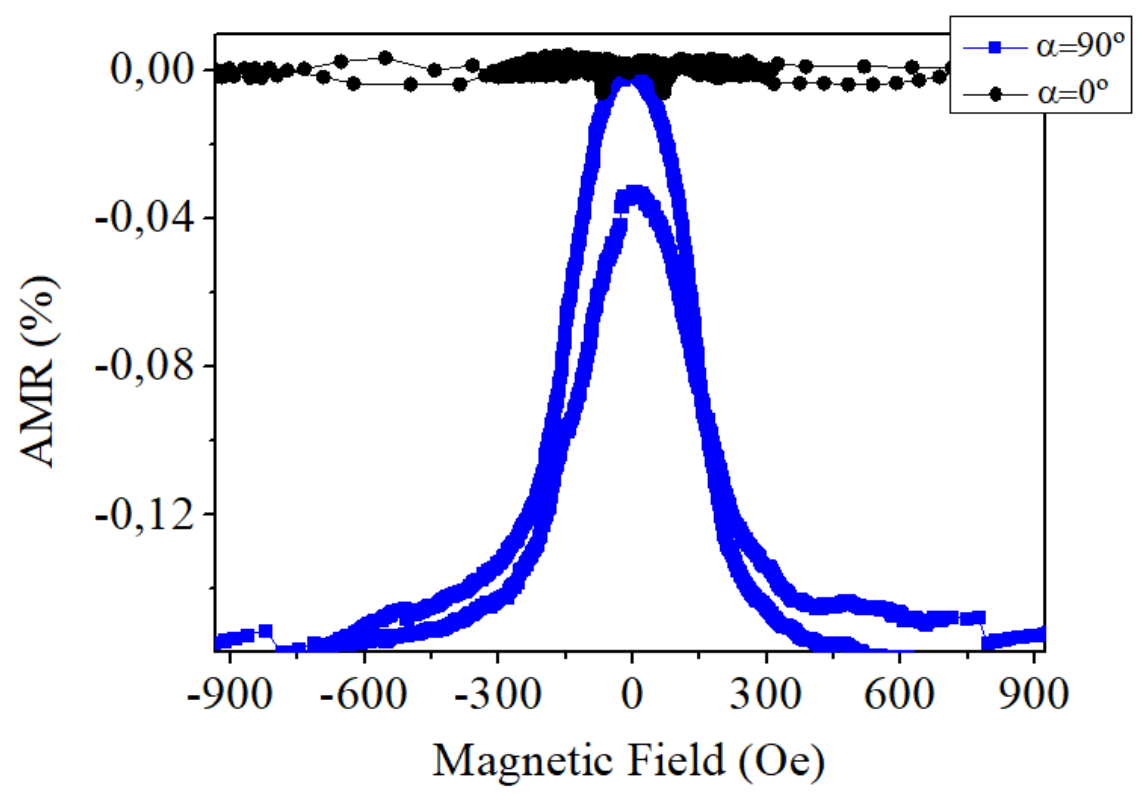

FIG. 88: Comparison of AMR measurements of a $35 \mathrm{~nm}$ thick FeCoB nanostripe with an external magnetic field applied parallel (black curve) and perpendicular (blue curve) .

Magnetoresistance measurements are performed in the FeCoB nanostripe in presence of SAW's with an external magnetic field applied parallel to the long axis of the nanostripe $(\alpha=0)$. As in the case of $\mathrm{Ni}$, SAW's induce a non-resistive voltage that is perceived as a jump in the resistance value in the AMR curve due to the acoustoelectric effect and the increase in temperature. In figure 89, AMR curves in absence and in presence of a SAW $+19 \mathrm{dBm}$ power signal are compared and displayed in terms of the percentage change of their resistance. It is observed that the application of the SAW's generate an asymmetry in the AMR curve with respect to the external magnetic field (this will be discussed in chapter IX, section B.). However, measurements of magnetoresistance do not show any decrease in the coercive fields at any power in this setup geometry.

The computation of this effective field originated by surface acoustic waves propagating along the ferromagnet can be found in the work of [Dreher, 2012]. A brief theoretical calculation of the effective field is exposed in this section. First, the dynamic, magnetoelastic contribution of the SAW's to the free-energy density reads as ([Chikazumi, 1997]): 


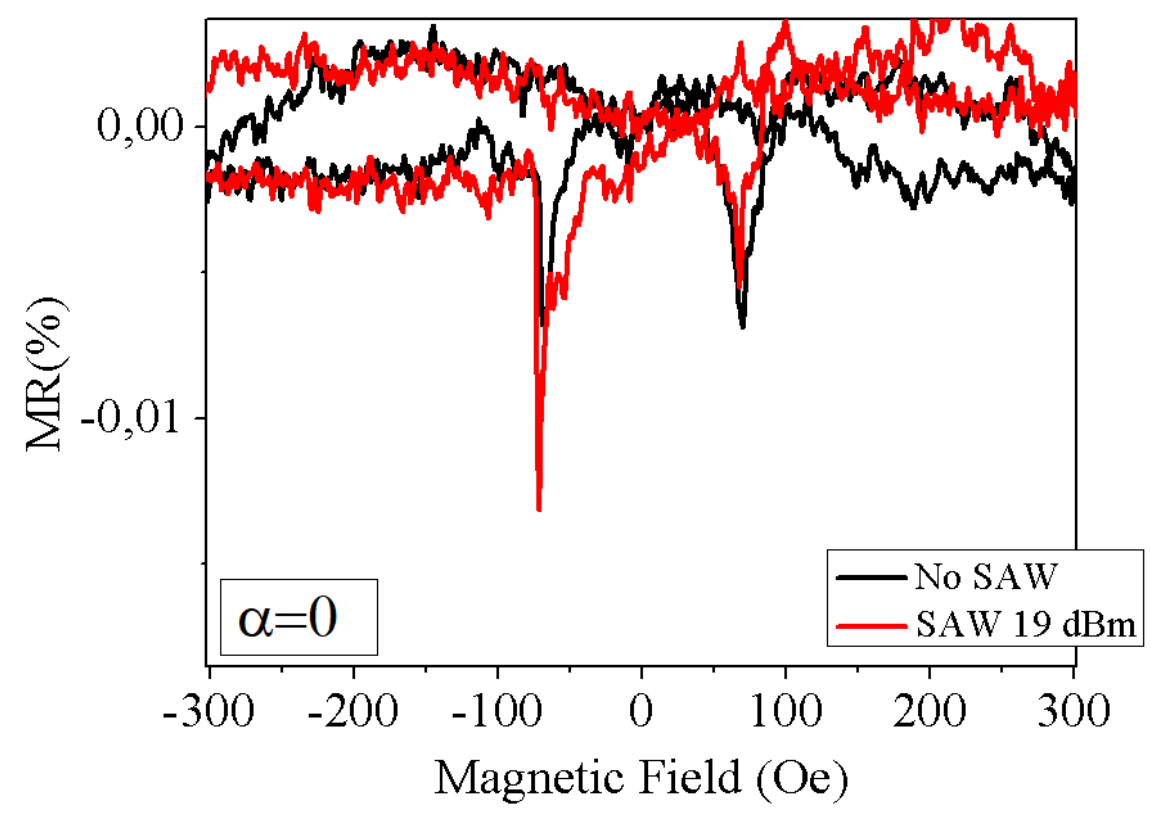

FIG. 89: Comparison of AMR measurements of a $35 \mathrm{~nm}$ thick FeCoB nanostripe with (red curve) and without (black curve) SAW's applied.

$$
E^{m-e}=b_{1}\left[\epsilon_{i j} \cdot m_{i} m_{j}\right]
$$

where $i$ and $j$ are the subscripts for the spatial coordinates, $\epsilon_{i j}=\frac{1}{2}\left(\frac{\partial u_{i}}{\partial x_{j}}+\frac{\partial u_{j}}{\partial x_{i}}\right)$ are the strain tensor components, $u_{j}$ are the components of the mechanical displacement field, $b_{1}$ is the magnetoelastic coupling constant and $\mathbf{m}=\mathbf{M} / M_{s}$ is the direction of magnetization. Equation (21) is valid for cubic symmetry of the ferromagnetic layer, as well as for a polycrystalline film. In this specific case, this expression can be further simplified taking into account that a Rayleigh wave only has $\epsilon_{x x}, \epsilon_{x z}$ and $\epsilon_{z z}$ as non-zero strain components.

From the magneto-elastic contribution to the free energy density, it is possible to compute the effectic magnetic field that generates the acoustic wave:

$$
\mu_{0} \mathbf{h}^{m-e}=-\nabla_{\mathbf{m}} E^{m-e}
$$

where $\nabla_{\mathbf{m}}=\left(\frac{\partial}{m_{1}}, \frac{\partial}{m_{2}}, \frac{\partial}{m_{3}}\right)$ is the vector differential operator with respect to the components of $\mathbf{m}$. The expression of the effective magnetic field when $\mathbf{m}$ lies within the film plane 
is dependent on the orientation of the magnetization:

$$
\mu_{0} \cdot h^{m-e}(t)=-2 \cdot b_{1} \cdot \epsilon_{x x}(t) \cdot \cos (\theta) \cdot \sin (\theta)
$$

where $\theta$ is the equilibrium in-plane angle that forms the magnetization with the direction of propagation of the SAW's. It is necessary to take into account that $\theta$ depends on the compromise between the Zeeman energy and the in-plane anisotropy. In this specific case, figure 89 displays the magnetoresistance measurements for a $\mathrm{FeCoB}$ nanostripe where the magnetic field is applied parallel to the anisotropy direction $(\alpha=0)$ and they both match the propagation direction of the SAW's. Therefore, both magnetization and SAW's propagation direction are mostly parallel, making expression 23 tend to zero.

An experimental solid support for this argument can be found in [Weiler, 2011], where the absorption of SAW's by a ferromagnet is measured as a function of the orientation of an external magnetic field applied. Results show minimum absorption when the magnetic field is either parallel or perpendicular to the propagation direction of the SAW's and maximum absortion in the range of $\alpha=\left[30^{\circ}-60^{\circ}\right]$, which agrees with equation 23 .

\section{F. Conclusions}

This chapter was oriented to describe the effects that the application of a RF signal to the IDT of the SAW resonator generates in the magnetization of the magnetic nanostripes through the measurements of the magnetoresistance. In this chapter most measurements are carried out in Ni nanostripes and only ordinary AMR complete loops in presence of SAW's are performed with a $\mathrm{FeCoB}$ nanostripe.

The first consequence observed after the application of a RF signal to the SAW resonator is an immediate jump of the resistance of the nanostripe. This sudden increase depends on the magnitude of the power applied to the SAW resonator and it is related to the acoustoelectric effect and an increase in temperature $(\Delta T)$. However, under the application of a DC current through the nanostripe it was not possible to compute the contribution of each effect to this increase in voltage (see Chapter IX, section A.). The calculation of the increase in 
temperature is performed under the application of an $\mathrm{AC}$ current. In that case, the increase in resistance is completely associated to $\Delta T$. Results show that under an application of a $+19 \mathrm{dBm}$ power signal, the increase in temperature in a Ni nanostripe is $\Delta T=10.9 \mathrm{~K}$.

Another effect on the magnetization of the Ni nanostripe caused by the SAW's is an additional anisotropy forced in the direction of propagation of the SAW's. This is observed in the magnetoresistance measurements, where, from saturation down to low fields, the slope of the decreasing curve is lower in the presence of SAW's. This effect can be described by the simulations, which show that in presence of SAW's as the field decreases from saturation, there are fewer y-oriented domains with wider x-oriented DW's separating them, which leads to a smaller slope in the initial reversible part of the loop and a higher remanence. The magnetoresistance curves measured in presence of SAW's also present a decrease in the coercivity proportional to the power of the signal applied to the resonator. This decrease is related to an enhancement of non-reversible transitions and related to the addition of energy to the DW's, favoring the DW movement. The enhancement of non-reversible transitions is tested by performing magnetorestance minor loops in presence of SAW's. When a minor loop (sweeping the magnetic field from saturation to low fields and reverse) becomes hysteretic is a sign of a non-reversible transition. Results show that in every case the minor loops become irreversible before in presence of SAW's than in absence of them. It can also be observed that the stress carried by the SAW's is able to trigger an irreversible magnetic transition even before the external magnetic field switches direction. This behaviour can be described through the simulations, where these irreversible jumps are generated by two $\mathrm{x}$-oriented DW's collapsing and dissapearing.

In order to test that the SAW's provide the DW with additional energy to perform the reversal process, we carry out current pulse measurements to assist de DW dynamics while SAW's are applied. The premise is that a single current pulse passing through one of the current lines, generates a large magnetic field in a local area in a defined time gap. This current pulse assists the reversal process, however in combination with the SAW's the assist process is assisted even more. Measurements of the resistance of the nanostripes are taken $100 \mathrm{~ms}$ before $\left(R_{1}\right)$ and $100 \mathrm{~ms}$ after $\left(R_{2}\right)$ the application of the current pulse. The increase in resistance $\left(\Delta R=R_{1}-R_{2}\right)$ is always larger in presence of SAW's, which implies a larger 
change in the magnetization towards the saturation stage, which implies an addition of energy to the DW's that carry out the reversal process.

Finally we measure the magnetoresistance in presence of SAW's of a FeCoB nanostripe, however, not all effects observed previously in the Ni nanostripe were present. The acoustoelectric effect generates an increase in the resistance of the nanostripe. However, no induced anisotropy nor decrease in the coercive fields were observed in this nanostripe. The reason proposed to explain this lack of effects is the preferential orientation of the magnetization, parallel to the propagation direction of the SAW's. 


\section{SAW'S-INDUCED VOLTAGE IN MAGNETOSTRICTIVE NANOSTRIPES.}

In this chapter we have focused on the different effects responsible for the emergence of induced voltages along the nanostripes under the application of the SAW's. These effects are firstly noticeable in the magnetoresistance measurementes carried out in the magnetic nanostripes. The first visible consequence in the magnetoresistance (MR) measurements due to the application of the SAW's is an increase in resistance. The additional voltage related to this increase in resistance is associated with the interaction between the SAW's and the mobile carriers, which is known as the acoustoelectric effect. This occurs because the free carriers in the nanostripe experience a net displacement due to the electrical and mechanical waves and it has been observed in a GaAs nanowire [Buyukkose,2014] and also observed recently in a FeCoB ferromagnetic stripe [Kawada,2019].

As it was stated in the introductory chapter, the application of SAW's to magnetic nanostructures has been proven to promote elastically driven ferromagnetic resonance (FMR) in thin films [Weiler, 2011], [Thevenard, 2014] and to pump spin current [Weiler, 2012] in ferromagnetic/non-magnetic bilayers. This spin pumping could be measured via inverse spin Hall effect (ISHE). We need to explore the possibility that the induced voltage in the magnetic nanostripe has this origin. However, there are more mechanisms that may be involved. For instance, the electrical potential that travels along the SAW's in the piezoelectric substrate can be shorted by the conductive nanostripe, which results in a RF current along the nanostripe. Such SAW-induced RF currents in the conductive nanostripe may give rise to dc voltages due to various mechanisms that result. During this chapter, we measure directly the voltage $\left(V_{D C}\right)$ induced by the SAW's in the magnetic nanostripes to check which one of these mechanisms may be the origin of it. Finally we expose intentionally the nanostripes to RF currents and mesure the resulting mixing voltage in order to compare it with $V_{D C}$. 


\section{A. Acoustoelectric Effect}

The application of the SAW's generates a voltage along the long axis of the nanostripe which we measure as an additional resistance in the magnetoresistance measurements. In order to understand this effect it is necessary to take into account that alongside the acoustic wave, under the application of a RF signal, the IDT of the SAW resonator generates an electromagnetic wave that propagates along the acoustic wave. When a semiconductor or a metal film is subject to the action of the SAW's, the free carriers (electrons) experience an unidirectional net displacement due to the action of the travelling mechanical and electrical waves. This is known as the acoustoelectric effect, and it has been observed in GaAs nanowires [Buyukkose,2014] and also observed recently in a $\mathrm{FeCoB}$ ferromagnetic stripe [Kawada,2019]. This effect is due to the transfer of linear momentum from the SAW's to the free electrons, which in a metal results in a build-up voltage as schematized in figure 90. Therefore, when using a current source for the measurement of the magnetoresistance, the total voltage required to deliver the requested current depends on the resistance of the nanostripe and on the acoustoelectric voltage $\left(V_{A E}\right)$ generated by the SAW's. The corresponding electric circuit is depicted in figure 91 . The total voltage measured is the voltage drop in the resistive nanostrip plus $V_{A E}$.

The magnitude of the acoustoelectric effect is exposed when we compare magnetoresistance measurements in presence of SAW's with the voltage connections in opposite contact pads (opposite DC current). Since the acoustoelectric effect generates a displacement of charge always in the same direction, the voltage applied by the current source depends on whether $V_{A E}$ opposes or favors the DC current flow. In figure 90 we compare the magnetoresesistance curves in a Ni nanostripe in presence of SAW's (19 dBm signal power), with a DC current amplitude of $50 \mu A$ in opposite directions. The acoustoelectric voltage can be calculated from the offset difference in the magnetoresistance measurements measured with opposite DC currents. An equivalent circuit to our experiment in presence of the acoustoelectric effect is schematized in figure 91, where the nanostripe is represented by the load $R$ with a potential drop of $V_{R}$, along with the current source, the additional acoustoelectric voltage $\left(V_{A E}\right)$ and the voltage measured $\left(V_{m}=V_{R}+V_{A E}\right)$. The resistance value measured is the ratio $V_{m} / I_{D C}$. 
a)

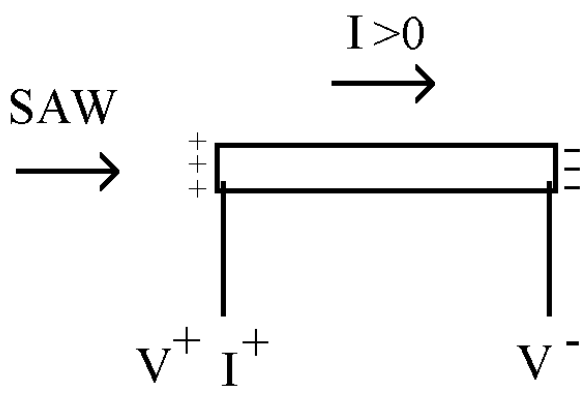

b)
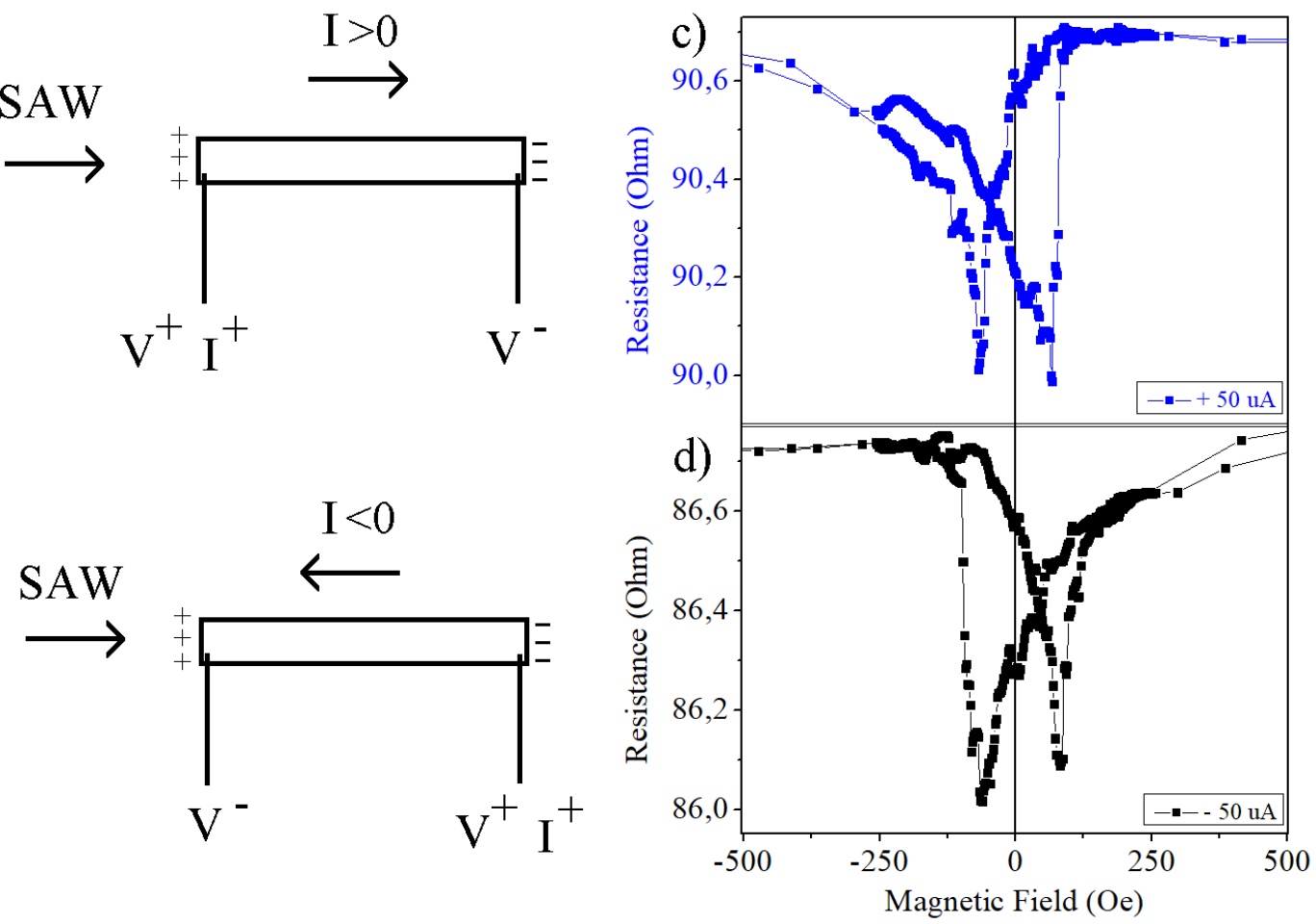

FIG. 90: a), b) Sechematics of the nanostripe, its connections and the defficiencty of electrons originated at the ends. c), d) Comparison of MR curves in Ni nanostripes, with a RF applied signal of $19 \mathrm{dBm}$ of power with a DC current of an amplitude of $50 \mu \mathrm{A}$ and opposite directions.

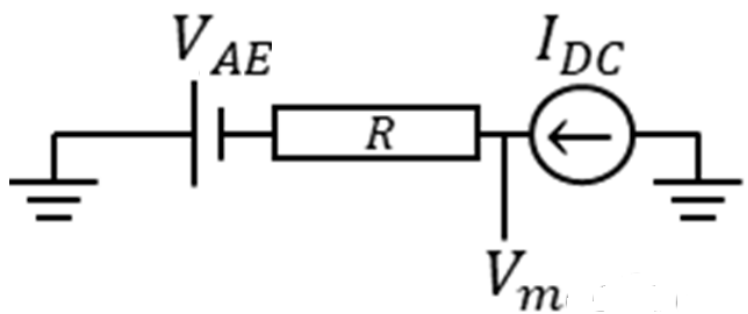

FIG. 91: Schematics of the equivalent circuit of the nanostripe in presence of the acoustoelectric effect. $V_{E A}$ represents the acoustoelectric voltage generated by the charge carriers.

Let us compare the resistance values at saturation in figure 90 for opposite DC currents flow directions. At $90 \mathrm{c}$ ) the acoustoelectric current opposes the DC current, whereas in 90 d) they flow at the same direction. Therefore, the equations for the circuit can be written:

1) $V_{A E}+V_{R}=50 \mu A \cdot R$ 


$$
\text { 2) }-V_{A E}+V_{R}=50 \mu A \cdot(R+3.9 \Omega)
$$

Solving the system, we obtain $V_{A E}=95 \mu V$, which is the additional voltage induced by the acoustoelectric effect in the Ni nanostripe at $19 \mathrm{dBm}$.

Aditionally to the increase in resistance that we relate to the acoustoelectric effect, when we measure the magnetoresistance curves of magnetic nanostripes in presence of SAW's, the MR curves present an asymmetry with respect to the external magnetic field. Results show a clear correlation between the asymmetry and the power of the signal. Figure 92 displays the MR curves for different powers, where it is clear how the larger the power applied, the more pronounced the asymmetry.

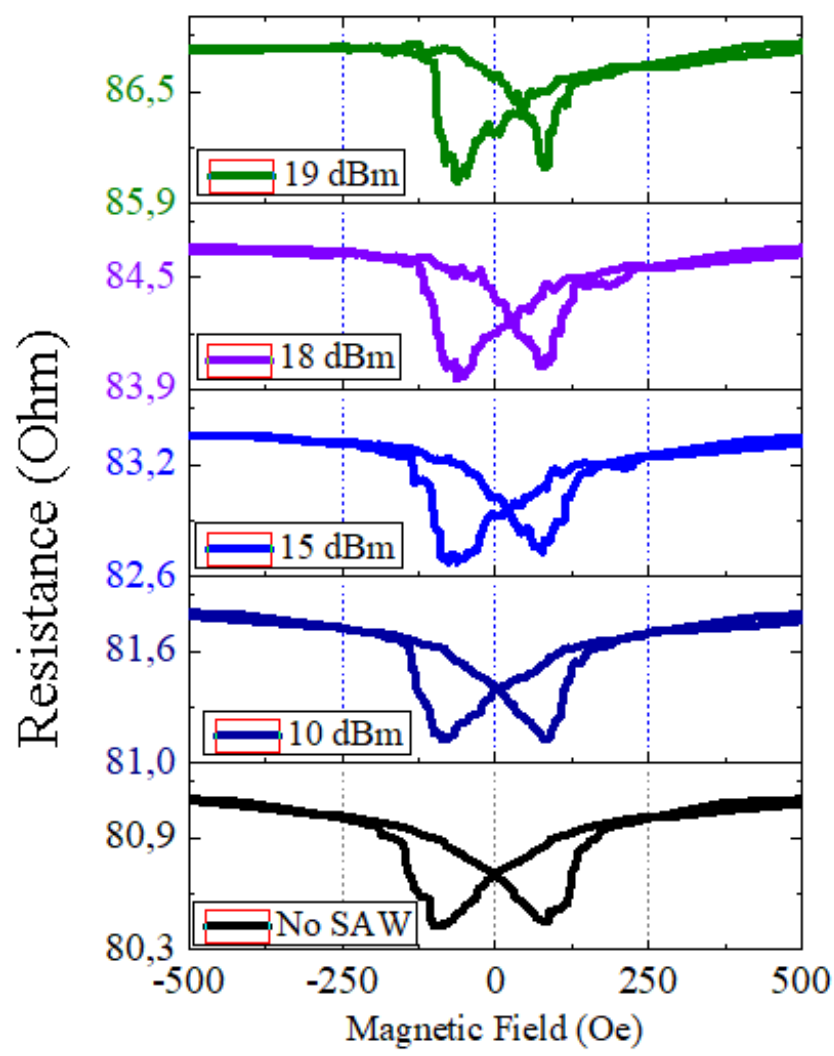

FIG. 92: Comparison of MR measurements in presence of SAW's for different powers of the applied to the resonator. The asymmetry becomes gradually more prominent as the power is increased.

This distorsion is associated to another type of voltage that the SAW's induce in the magnetic nanostripes. The effect of this voltage in the magnetoresistance measurements is 
also dependent on whether this induced voltage opposes or not the DC current flow direction. That is the reason behind the inversion in the asymmetry in figures 90 c) and d). Since it is interesting the relation between the induced voltage and the DC current applied, we perform several measurements of magnetoresistance in presence of SAW's for different amplitudes of the DC current. Figure 93 displays the comparison between MR measurements with a signal power of $19 \mathrm{dBm}$ applied to the SAW resonator, for two DC currents amplitudes flowing through the Ni nanostripe $(50 \mu A$ and $500 \mu A)$. Results show that the asymmetry is enhanced as the DC current decreases. This occurs because the voltage induced by the SAW's has always the same magnitude (at a fixed RF signal power) with independence of the DC current applied by the current source. When the DC current amplitude is lower, the resistive voltage measured is also low, which makes the contribution of the induced voltage to the final measurement comparatively more relevant, therefore, enhancing the asymmetry.

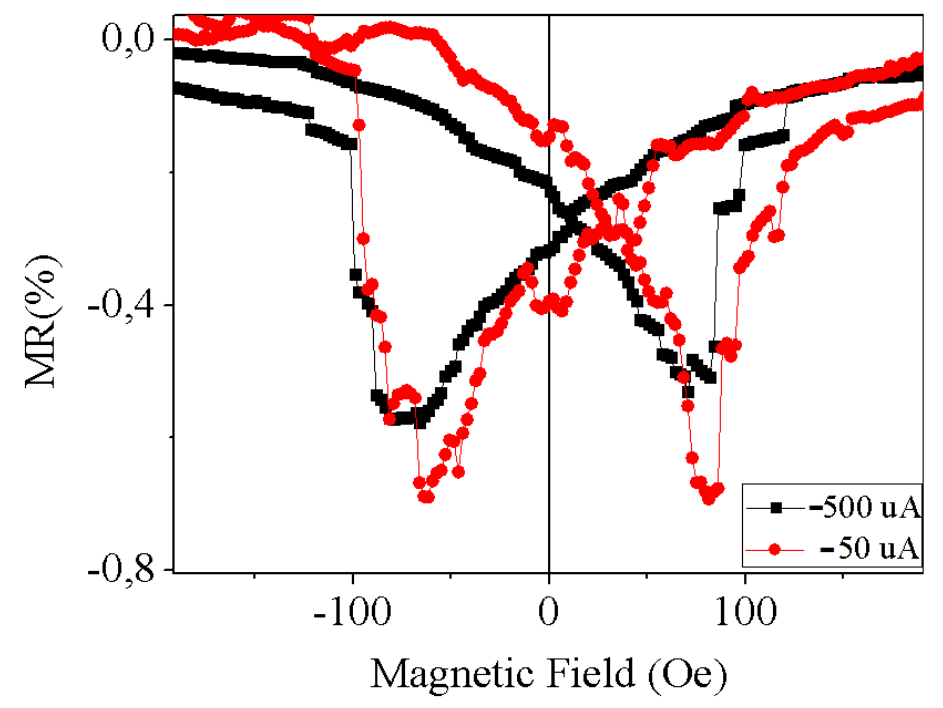

FIG. 93: Plots of the AMR curves in presence of SAW's with $19 \mathrm{dBm}$ power applied to the SAW resonator. Measurements are performed for different amplitudes of the DC current applied to the nanostripe, $50 \mu A$ (red curve) and $500 \mu A$ (black curve). 


\section{B. Direct measurement of the Induced Voltage in the Nanostripes}

We now present the results of the measurements of the induced voltage $\left(V_{D C}\right)$ due to the SAW's in absence of a DC current flowing through the nanostripe. Here we expect to measure the contributions from the acoustoelectric effect, as well as the voltage that the SAW's induce in the magnetic nanostripes and originate the asymmetry in the magnetoresistance measurements. In order to separate both effects, we fabricate devices with nanostripes of $\mathrm{Au}$ in addition to the devices with $\mathrm{Ni}$ and $\mathrm{FeCoB}$ nanostripes. At this point, we measure directly the voltage induced by the SAW's in the nanostripes. A SEM image of one those nanostripes is presented in figure 94, where the schematics of the setup experiment is drawn on top of the image and the directions of the SAW and the magnetic field are depicted. A Lock-in amplifier is used to perform the measurements.

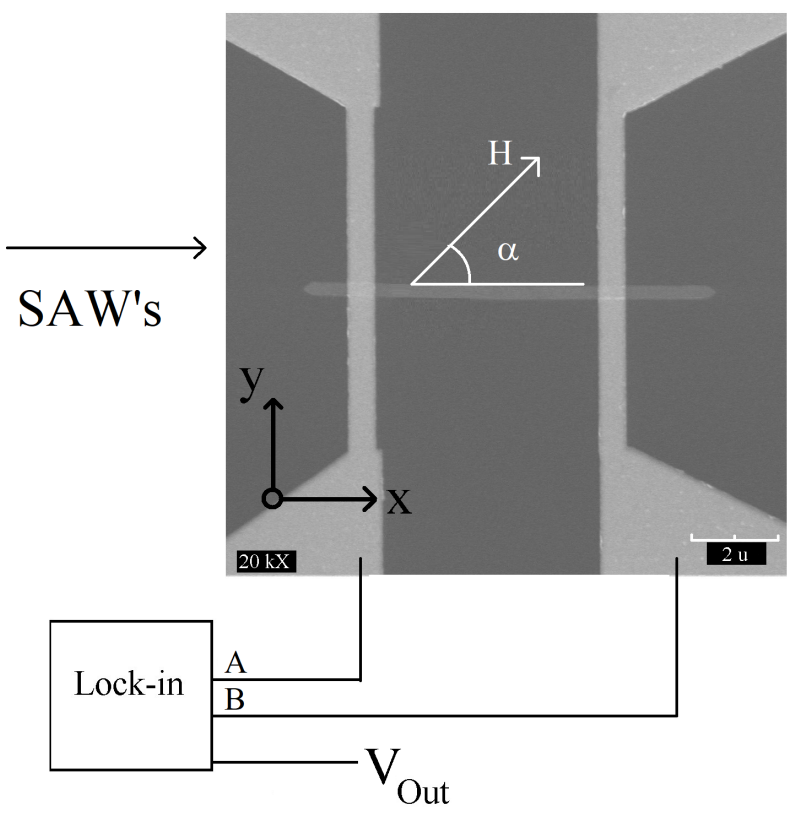

FIG. 94: SEM image of a Ni nanostripe. The schematics of the experiment setup is depicted over the image. SAW's propagation and magnetic field direction are depicted.

In every measurement, the RF signal is set to the Rayleigh frequency of the SAW resonator and the lock-in amplifier (LIA) is locked at the modulating frequency of either $1 \mathrm{kHz}$ or 400 Hz. In order to the LIA to be able to provide a DC signal, this modulation is necessary since its performance relies on the orthogonality of sinusoidal functions. The LIA takes the input 
signal, multiplies it by a reference signal and integrates over a specified time. A screenshot from the oscilloscope that shows the RF modulated signal applied to the SAW resonator is presented in figure 95.

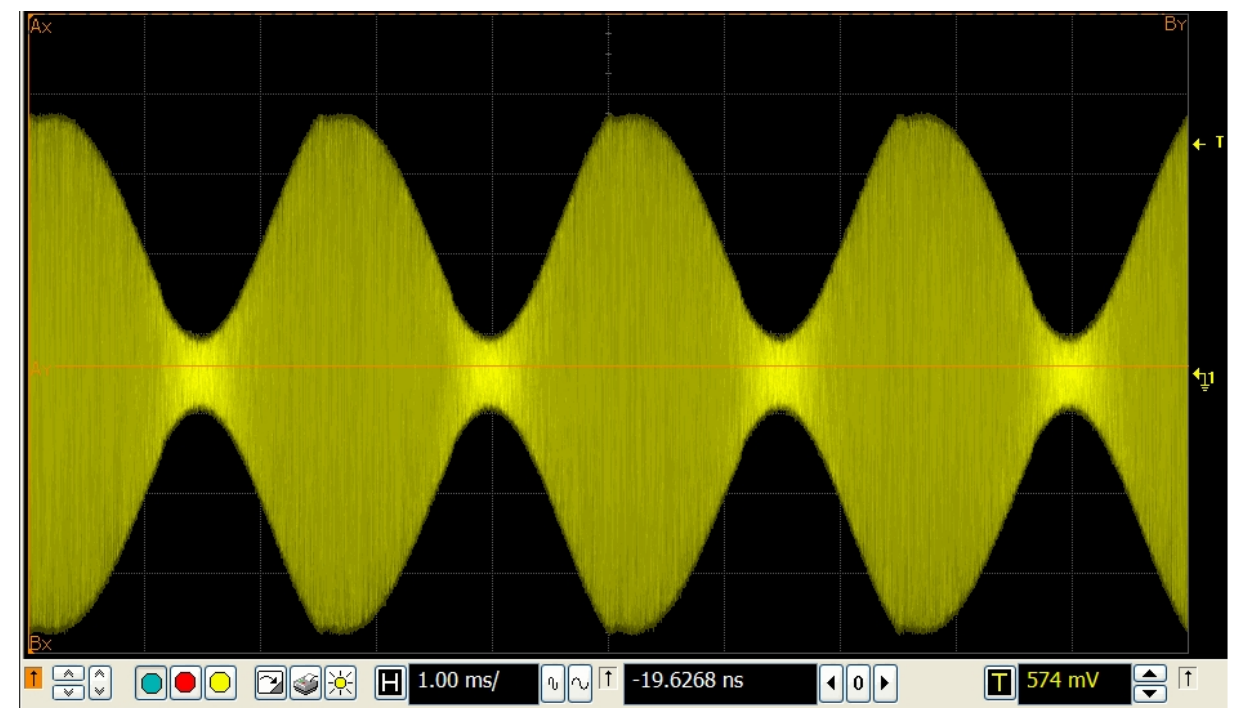

FIG. 95: Screenshot from the oscilloscope connected to the SAW resonator. The original carrier signal at the Rayleigh frequency is typically $\sim 1.2 \mathrm{GHz}$. The modulation envelope has frequency of $400 \mathrm{~Hz}$ and a depth of $80 \%$.

\section{Induced voltage measurements in Au nanostripe}

Straintronics devices were fabricated with nanostripes made out of Au with a buffer of $\mathrm{Cr}$ and the same structure as the contact pads (Cr $20 \mathrm{~nm} / \mathrm{Au} 120 \mathrm{~nm}$ ). The dimensions of the $\mathrm{Au}$ nanostripe are $10 \mu \mathrm{m} \times 300 \mathrm{~nm} \times 120 \mathrm{~nm}$. The RF signal (see figure 95) is applied to the SAW resonator and the voltage induced is measured in the contact pads. Since this is performed in a non-magnetic nanostripe, we assume that the induced voltage $\left(V_{D C}\right)$ does not depend on the external magnetic field. This is indeed the case as shown in figure 94. Nevertheless, the acoustoelectric effect induces a $V_{D C}$ signal measured in the contact pads. This voltage is measured as a function of an external magnetic field $\left(H_{\text {ext }}\right)$ for different powers. However, $V_{D C}$ is constant under the application $H_{\text {ext }}$. We assume that 
this contribution from the acoustoelectric effect to $V_{D C}$ is analogous to the contribution in the magnetic nanostripes.

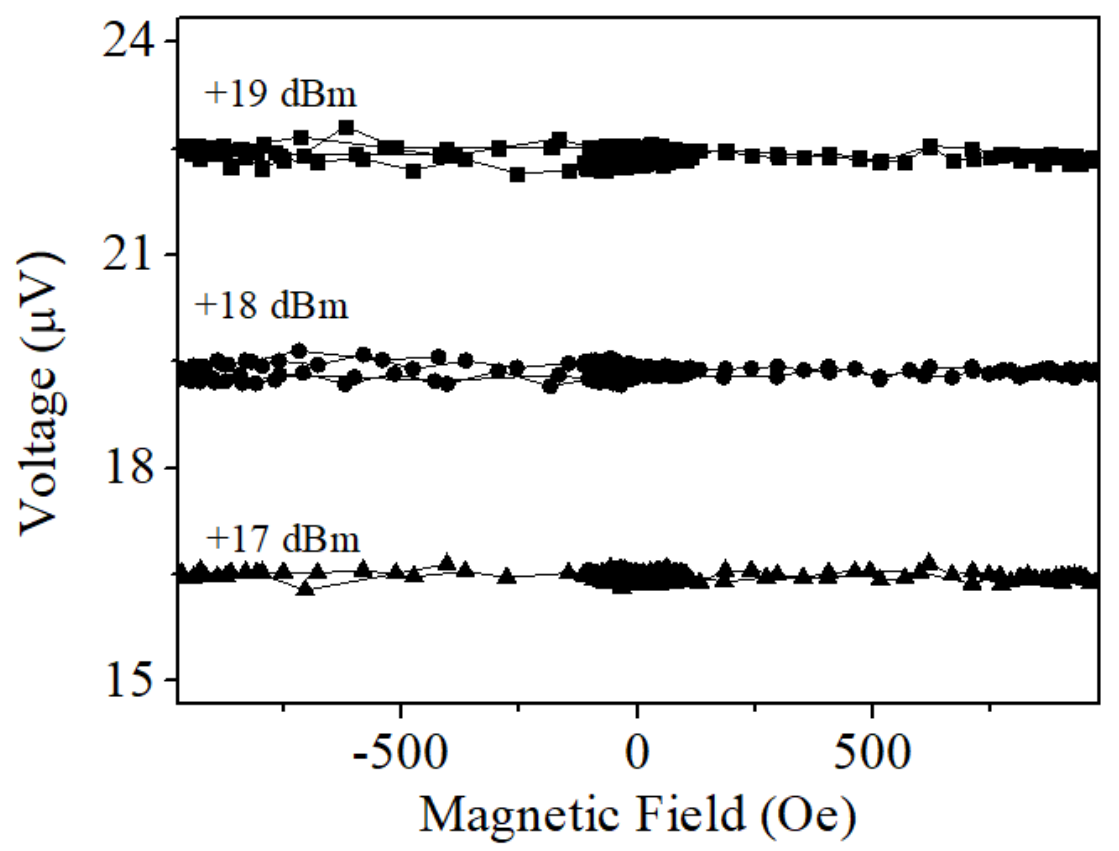

FIG. 96: Voltage $\left(V_{D C}\right)$ induced by the SAW's in a Au nanostripe for different RF signal powers applied to the SAW resonator. Measurements are performed as a function of an external magnetic field applied parallel to the long axis of the nanostripe $(\alpha=0)$.

\section{Induced voltage measurements in magnetic nanostripes}

$V_{D C}$ measurements are performed in devices with magnetic nanostripes of the materials aforementioned during this work, which are $\mathrm{Ni}$ and FeCoB. The experimental setup has been shown in figure 94 and $V_{D C}$ is measured as a function of an external magnetic field for different powers of the RF signal applied to the SAW resonator. As we have mentioned before, the voltage that the SAW's induce in the all type of nanostripes come from the acoustoelectric effect. However, SAW's also generate an additional voltage in the magnetic nanostripes, which depends on the magnetization state of the nanostripes and it is the responsible for the asymmetry observed in the magnetoresistance curves. 


\section{Induced Voltage in Ni Nanostripes}

The voltage $\left(V_{D C}\right)$ induced by the SAW's in the Ni nanostripe depends on the external magnetic field. The schematics of the experimental setup for the $V_{D C}$ measurements were presented in figure 94. The input signal applied to the SAW resonator is set to its Rayleigh frequency and modulated at $1 \mathrm{kHz}$ with $80 \%$ depth. Measurements are performed for different powers applied through the signal generator and the results are displayed in figure 97.

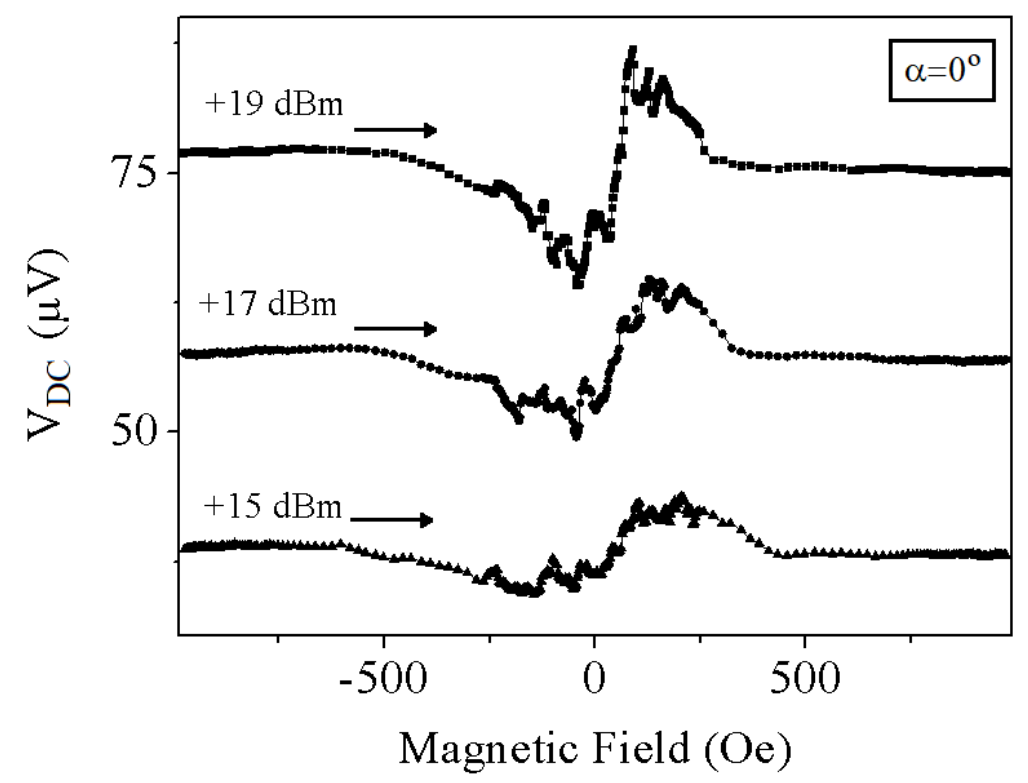

FIG. 97: $V_{D C}$ measurements in the $35 \mathrm{~nm}$-thick Ni nanostripe for different powers of the signal applied to the resonator. The external magnetic field is applied parallel to the long axis of the nanostripe $(\alpha=0)$. Black arrows indicate the direction of the magnetic field sweeping.

$V_{D C}$ is the additional voltage that the SAW's induce in the Ni nanostripe and distorts the AMR measurements, as it can be seen in figure 98. Therefore, this induced voltage is the reason why the AMR is asymmetric in presence of SAW's and why this asymmetry is enhanced by reducing the amplitude of the DC current applied, since the lower the DC current amplitude, the larger relative contribution of the non-resistive voltage to the AMR measurement. In figure $98 \mathrm{a}$ ), AMR is measured by applyig a $50 \mu \mathrm{A}$ amplitude current along the $\mathrm{Ni}$ nanostripe and probing the voltage at its ends. By adding up $V_{D C}$ to the AMR measurements in absence of SAW's, the distorted AMR in figure 98 a) is perfectly 
reproduced. It can also be observed, that for an $80 \%$ depth modulated $\mathrm{RF}$ signal of +19 $\mathrm{dBm}$, the offset voltage measured of $V_{D C}$ is $77 \mu \mathrm{V}$. This matches perfectly with the $80 \%$ of the acoustoelectric voltage computed in section $\mathrm{A}\left(V_{A E}=95 \mu V\right)$.

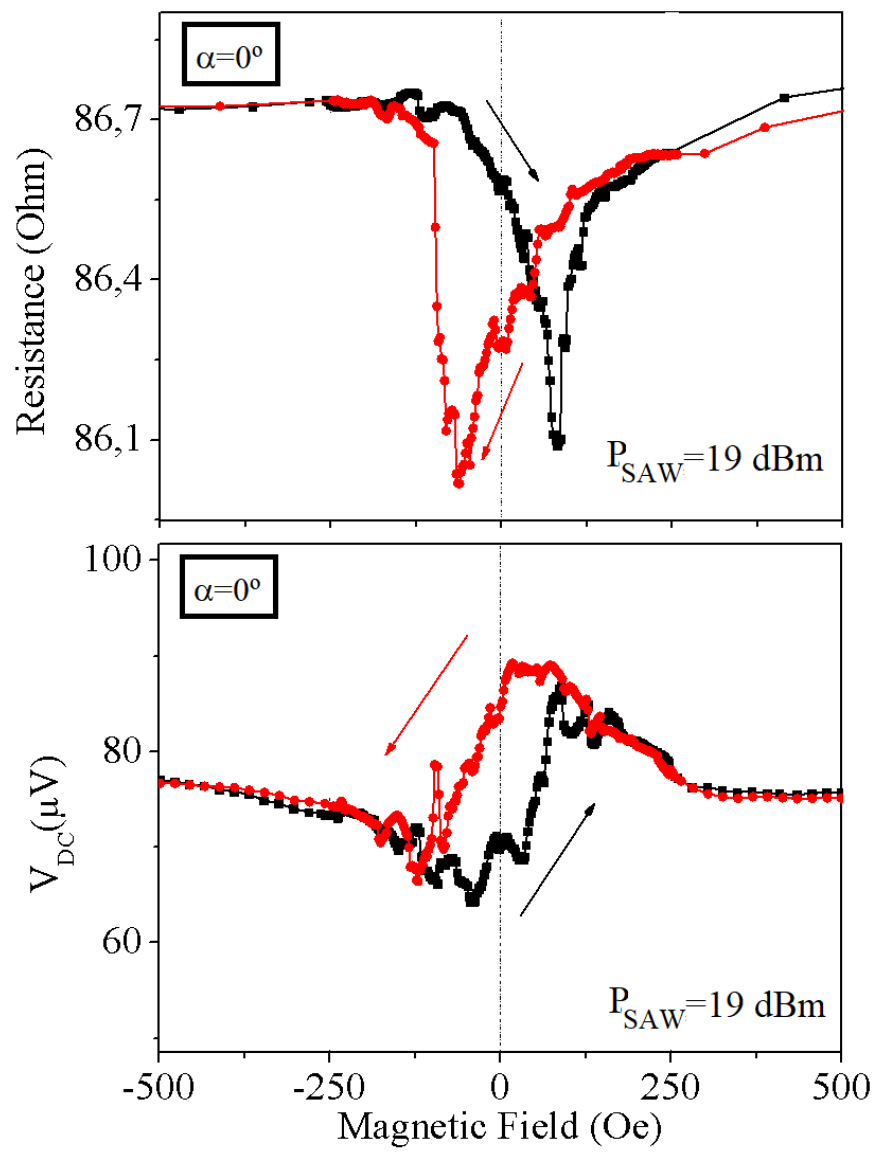

FIG. 98: a) AMR measurements of the Ni nanostripe in presence of SAW's. b) Induced-by-theSAW's voltage in the Ni nanostripe. Color of the curves are dependent on the sweeping direction of the $\mathrm{H}$ field. Both measurements are performed with an external $\mathrm{H}$ applied parallel to the long axis of the nanostripe.

In figure 98 it is observed that $V_{D C}$ signal is correlated to the AMR measurement. It is clear that the main features of the $V_{D C}$ signal appear when the reversal process of the nanostripe takes place. In order to describe the mechanism that give rise to $V_{D C}$, we measure $V_{D C}$ with different orientations of the $\mathrm{H}$ field and check if this correlation repeats. In figure 99, we present $V_{D C}$ with an external magnetic field applied perpendicularly to the long axis of the nanostripe $\left(\alpha=90^{\circ}\right) . V_{D C}$ at low fields has a similar shape to the signal obtained in figure 97 with $\alpha=0$, however, at saturation the induced voltage in this case are not 
equal. The amplitude of $V_{D C}$ at this orientation diminishes noticeably compared to the measurementes in figure 97, this is experienced ocasionally when changing the orientation of the magnetic field, since that involves removing the sample from the experimental setup and wire-bonding again.

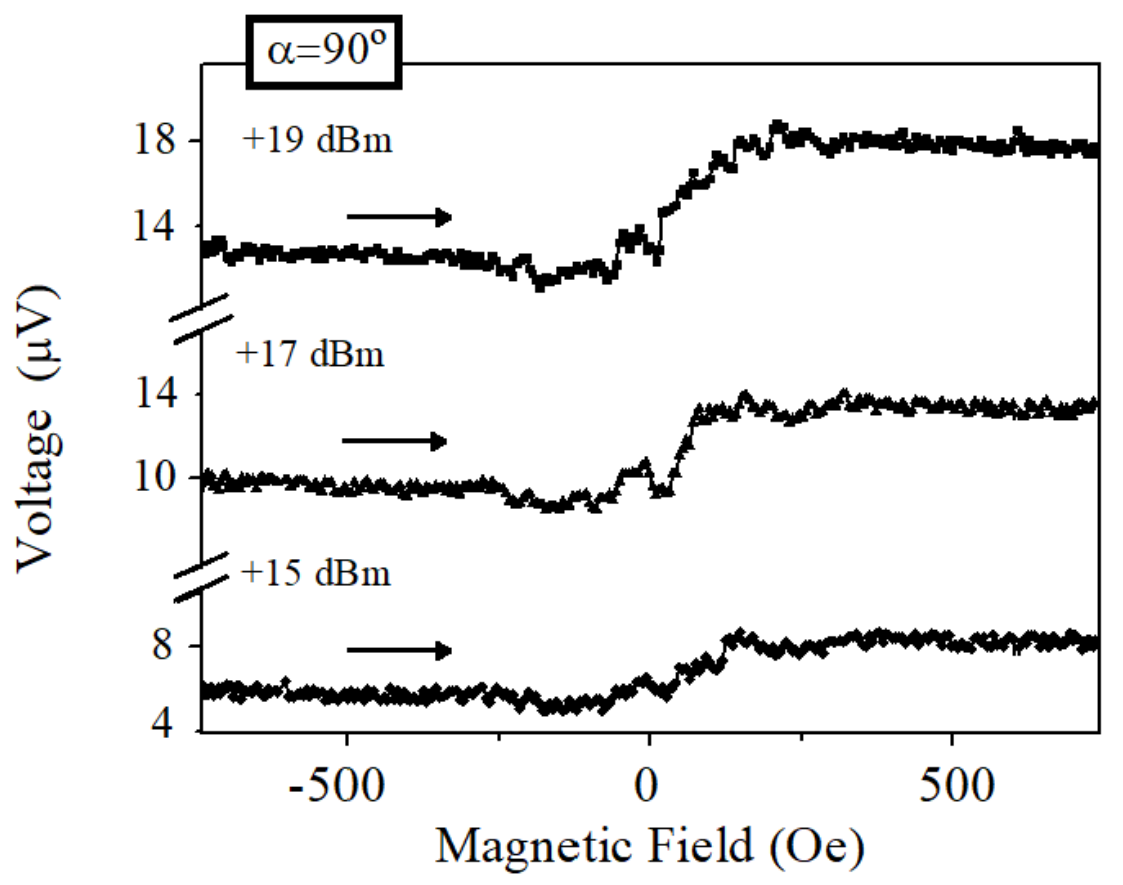

FIG. 99: $V_{D C}$ measured in the $35 \mathrm{~nm}$-thick Ni nanostripe for different powers of the signal applied to the SAW resonator. The external magnetic field is applied perpendicularly to the long axis of the nanostripe $\left(\alpha=90^{\circ}\right)$.

This induced voltage is responsible for the asymmetry found in the AMR measurement at $\alpha=90^{\circ}$ as it can be observed in figure 100 . Once again, $V_{D C}$ present its main features when reversal process occurs, therefore we can state that the $V_{D C}$ signal generated by the application of the SAW's is dependent on the magnetization state of the nanostripe, since the variation of the curve appears when the reversal process takes place, whereas at saturation $V_{D C}$ signal remains constant.

In order to stablish the origin of the induced voltage signal, it is necessary to take into account the electric field that propagates along the SAW's when they travel in a piezoelectric substrate. This RF electric field may generate an electrical RF current in the conductive nanostripe which may give rise to a dc voltage due to several mechanisms because of the 

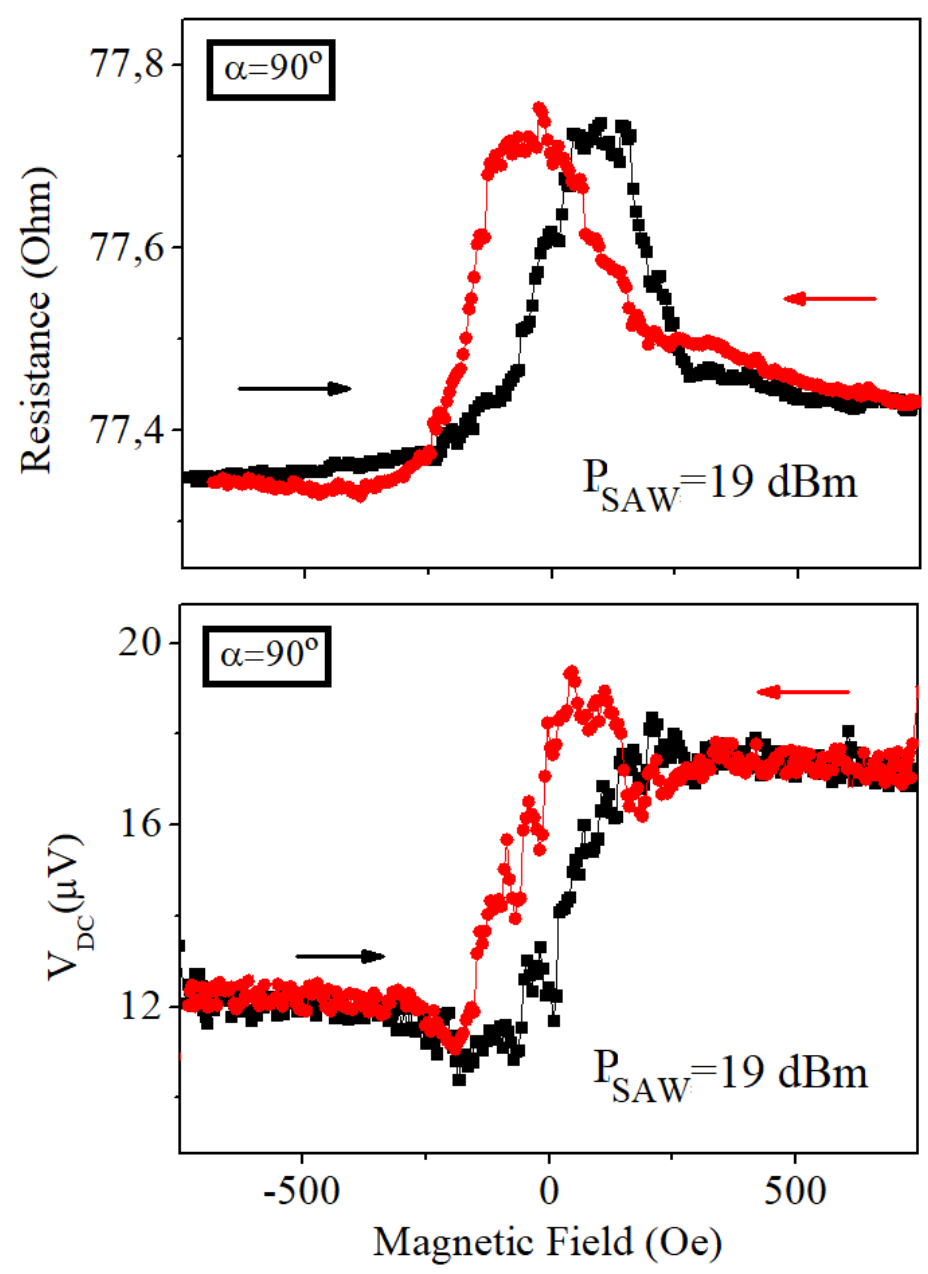

FIG. 100: a) AMR measurements of the Ni nanostripe in presence of SAW's. b) Induced-by-theSAW's voltage in the Ni nanostripe. Color of the curves are dependent on the sweeping direction of the $\mathrm{H}$ field. Both measurements are performed with an external $\mathrm{H}$ applied perpendicular to the long axis of the nanostripe

mixing of the resonant (and non-resonant) magnetization precession and the RF current [Liu, 2011]. The application of a RF current in ferromagnetic/non-magnetic bilayer is capable to force the precession of the spins in the ferromagnet by different mechanisms. In the first place, a longitudinal RF current flowing through the non magnetic metal $\left(J_{C}\right)$ can inject a transversal spin current $\left(J_{S}\right)$ from the non magnetic layer into the ferromagnetic layer (spin Hall effect), which forces the precession of the spins in the ferromagnet when the frequency of the RF current reaches the resonant condition. Plus, the RF current also generates a RF Oersted field, which also is capable to force the spin precession in the ferromagnet at the 
resonant condition. This resonant precession of the spin causes an oscillation of the bilayer resistance due to the anisotropic magnetoresistance (AMR). Mixing of this resistance with the RF current yields a dc voltage. However, we can discard these mechanisms as the origin for $V_{D C}$, since the functioning frequency of the SAW's is relatively low $(\sim 1.2 \mathrm{GHz})$, which requires a static external field so low that the resonant condition is reached when the nanostripe is not saturated. Furthermore, we only observe one resonance-like signal in every magnetic field sweeping instead of two, therefore we can exclude these mechanisms. Nevertheless, there is an additional effect that may have a contribution in the $V_{D C}$ signal of this experiment, and that is the non resonant spin rectification. This spin rectification effect due to the non resonant magnetization motion, yields a dc voltage associated with the magnetization reversal process that occurs in the sample [Zhu, 2011]. This voltage appears in general by the coupling of the dynamic current and the oscillating magnetization at non resonant condition. In order to assure the spin rectification origin to this $V_{D C}$ signal, we expose intentionally our nanostripes to a RF current and compare the measured $V_{D C}$ induced by the SAW's with the mixing voltage $V_{\text {mix }}$ induced by the RF current. The Ni nanostripe was connected to a bias tee as is shown in figure 101 in order to measure the de voltages induced by the RF current applied along the $\mathrm{x}$ axis by a signal generator.

A nanovoltimeter was used to record the dc voltage $V_{\text {mix }}$ and an oscilloscope is connected to the opposite contact in order to obtain the RF current amplitude that is flowing along the nanostripe. A rf current along x direction in the Pt generates an Oersted field $h_{1} \perp I_{r f}$ in the Ni nanostripe, which induces an oscillation in the mixing voltage due to the anisotropic magnetoresistance when the magnetization reversal process takes place. This mixing voltage originated by the motion of the magnetization is compared with the induced-by-the-SAW voltage measured previously. This is shown in figure 102 (blue curve), where the RF current frequency is the same as the SAW resonator functioning frequency $(\sim 1.2 \mathrm{GHz})$ and the external magnetic field is applied at $\alpha=0^{\circ}$ a) and $\alpha=90^{\circ} \mathrm{b}$ ).

It becomes clear the correlation between $V_{m i x}$ and $V_{D C}$ at different orientations of the $\mathrm{H}$ field, since both have similar shapes. In order to assure the non resonant spin rectification as the origin for the $V_{D C}$ signal, we study its angular dependance, which according to the work of [Zhu, 2011] yields a voltage: 


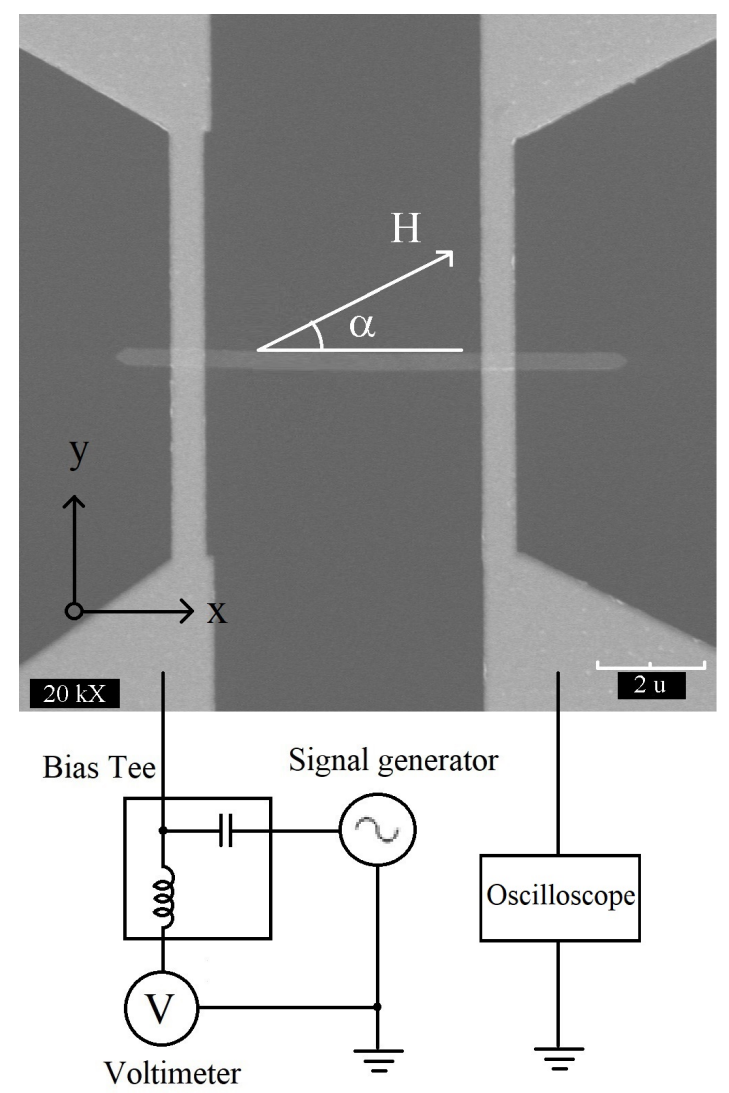

FIG. 101: Schematics of the configuration for the measurement of the mixing voltage, depicted on top of a SEM image of a Ni nanostripe.

$$
V_{S R}=-\frac{\Delta R j h}{H_{A}} \sin (\theta) \cos (\Phi)
$$

where $j$ is the dynamic current density excited by the SAW's flowing through the nanostripe, $\Delta R$ is the difference in resistance when the magnetization is parallel $\left(R_{\|}\right)$and perpendicular $\left(R_{\perp}\right)$ to the longitudinal direction of the nanostripe, $\theta$ is the angle between magnetization and direction of propagation of $j, h$ is the Oersted field generated by $j, H_{A}$ is the anisotropic field and $\Phi$ is the relative phase between the $j$ and the $h$ waves. The important information extracted from equation 24 is that the spin rectification voltage depends linearly on the sine of the angle that forms the magnetization with the direction of propagation of the SAW's. This fits perfectly with measurements presented in figure 102, where maximum values of the $V_{D C}$ signal are observed when the magnetization lies away from the long axis of the nanostripe. 

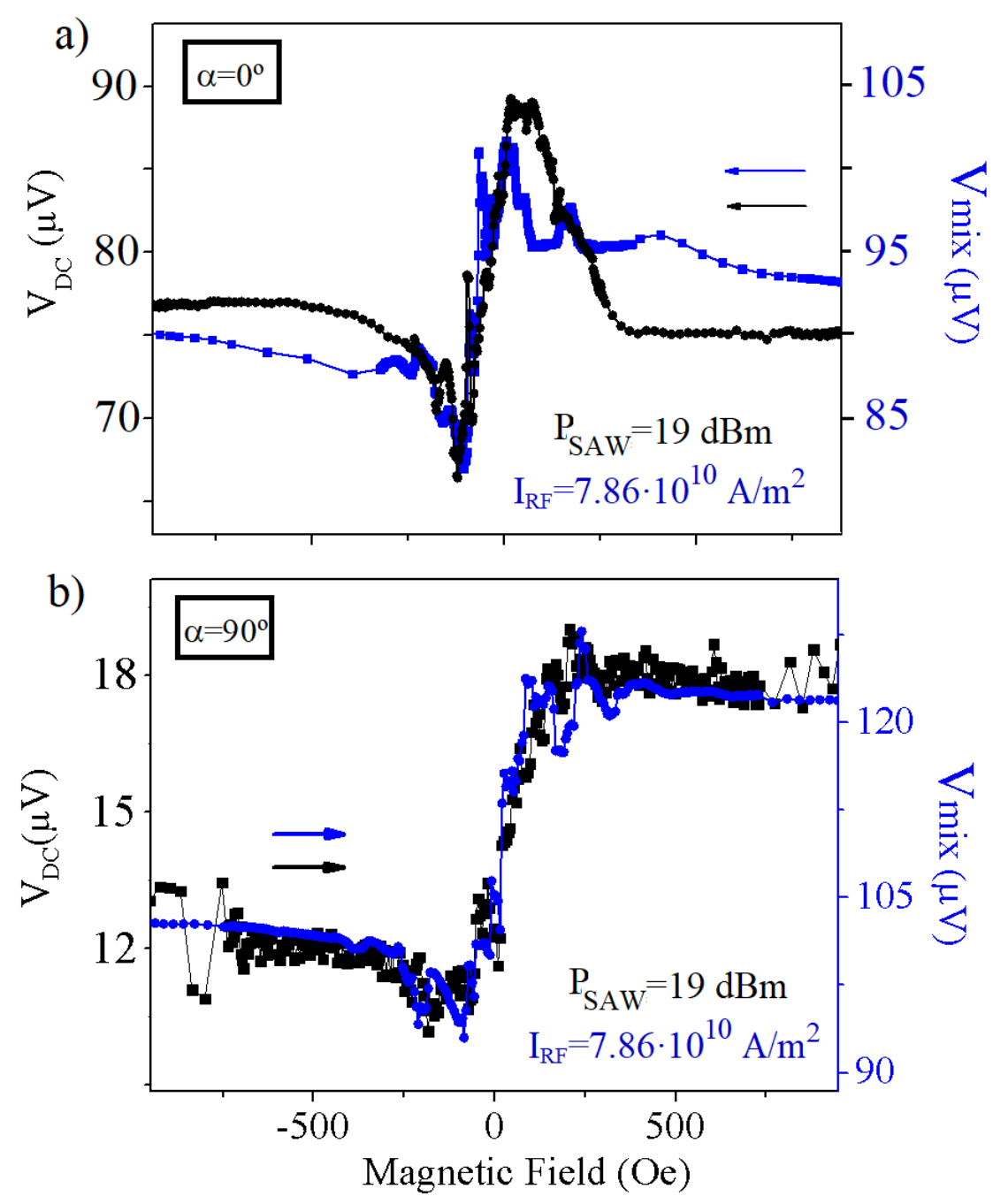

FIG. 102: Comparison of the voltage induced in the Ni nanostripe by the application of SAW's and the mixing voltage originated by the application of a RF current along the nanostripe when the external magnetic field is applied a) parallel and b) perpendicular to the long axis of the nanostripe.

Therefore we can conclude that the origin of the signal in presence of SAW's is due to the non resonant spin rectification that occurs when the reversal proces is taking place in the Ni nanostripe. 


\section{Induced Voltage in FeCoB Nanostripes}

$V_{D C}$ in presence of SAW's is measured in devices with FeCoB nanostripes in order to observe the effects of the SAW's in the AMR curves and to determine their origin. The dimensions of the FeCoB nanostripes are $2 \mu m \times 10 \mu m \times 35 \mathrm{~nm}$ and the experimental setup is described in figure 94. The signal applied to the SAW resonator is set to the Rayleigh frequency and modulated at $400 \mathrm{~Hz}$ with $80 \%$ depth. $V_{D C}$ measurements are presented in figure 103 where curves for RF signals of different powers applied to the SAW resonator are displayed as a function of the external magnetic field applied for an orientation of $\alpha=0^{\circ}$.

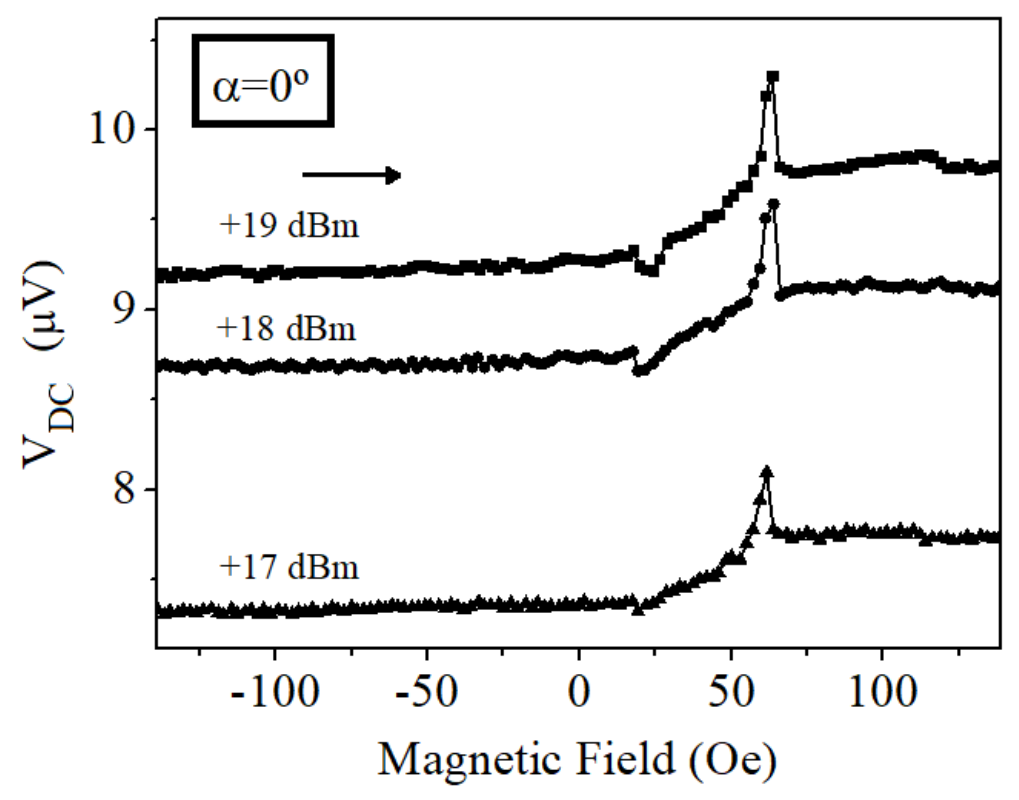

FIG. 103: a) Induced voltage in a FeCoB nanostripe $35 \mathrm{~nm}$ thick for different power of the RF signal and a magnetic field applied at $\alpha=0^{\circ}$. The arrow indicates the sweeping direction of the magnetic field.

Measurements show an antisymmetric lorentzian between +25 and +75 Oe in the upsweeping of the external magnetic field. It is interesting the comparison between the inducedby-the-SAW's voltage $\left(V_{D C}\right)$ and the AMR curves, which is displayed in figure 104 with an orientation of the external magnetic field of $\alpha=0^{\circ}$. In this figure, it becomes clear the distorsion of the AMR curve due to the $V_{D C}$, which is added to the AMR signal, originating the asymmetry. It can be observed also that $V_{D C}$ peaks when the reversal process takes 
place in the nanostripe.
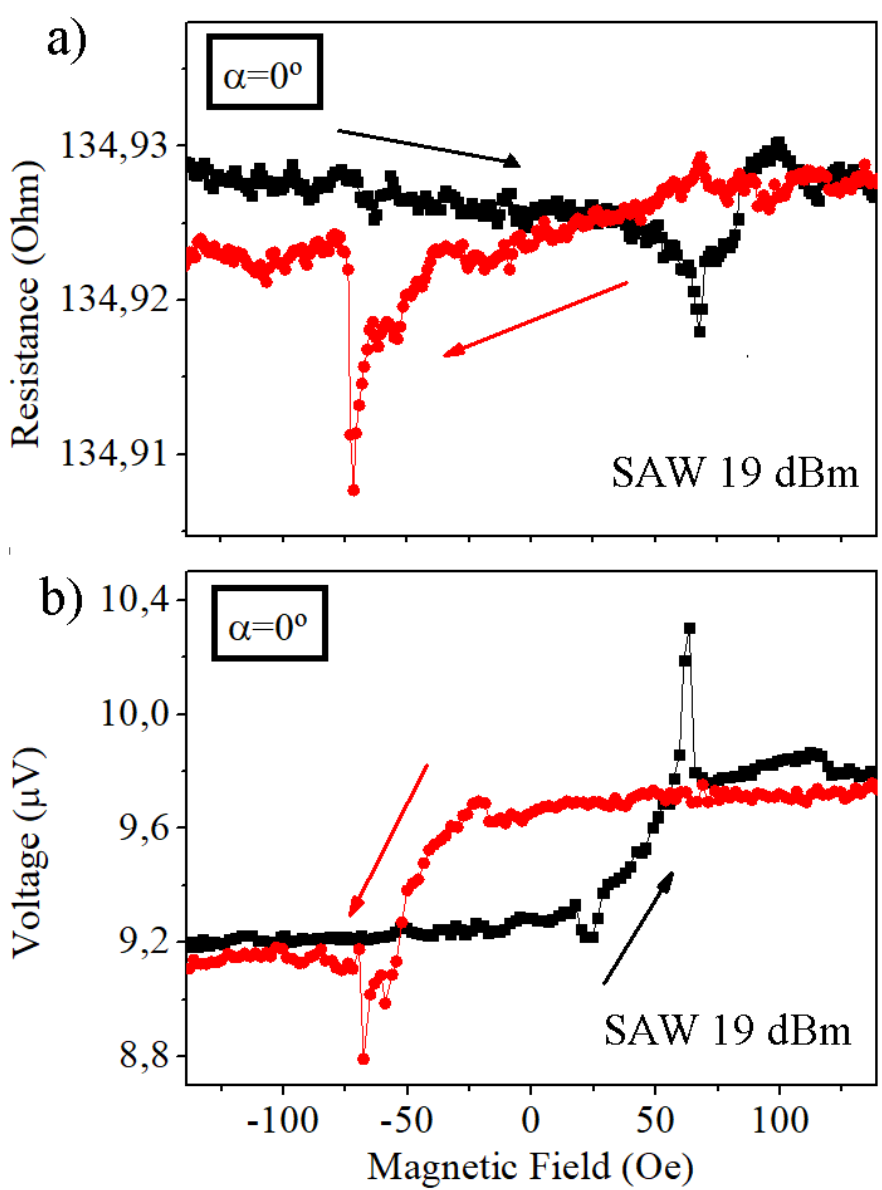

FIG. 104: Comparison between the AMR curve of the $35 \mathrm{~nm}$ thick FeCoB nanostripe when a 19 dbm power RF signal is applied to the SAW resonator (a) and $V_{D C}$ induced by the SAW's in the nanostripe as a function of the magnetic field (b). Black curves are plotted in the upsweeping of the $\mathrm{H}$ field, whereas red curves are plotted when the field is downswept.

In figure $104 \mathrm{~b}$ ) it can be seen that $V_{D C}$ presents a voltage offset which depends on the orientation of the magnetization at saturation. In order to obtain the possible contribution to the $V_{D C}$ signal from the spin rectification effect, ST-FMR measurements are performed. We force a RF current to flow along the FeCoB nanostripe (figure 101) and measure the mixing voltage $\left(V_{m i x}\right)$. Results are presented in figure 105 a), where $V_{\text {mix }}$ presents clear hysteresis related to the magnetization reversal and different offset voltages at saturation. By comparing qualitatively $V_{D C}$ and $V_{m i x}$ we can obtain the contribution of spin rectification to the $V_{D C}$, however we find that the peaks presented between +25 and +75 Oe are not 
reproducible by the application of a RF current.
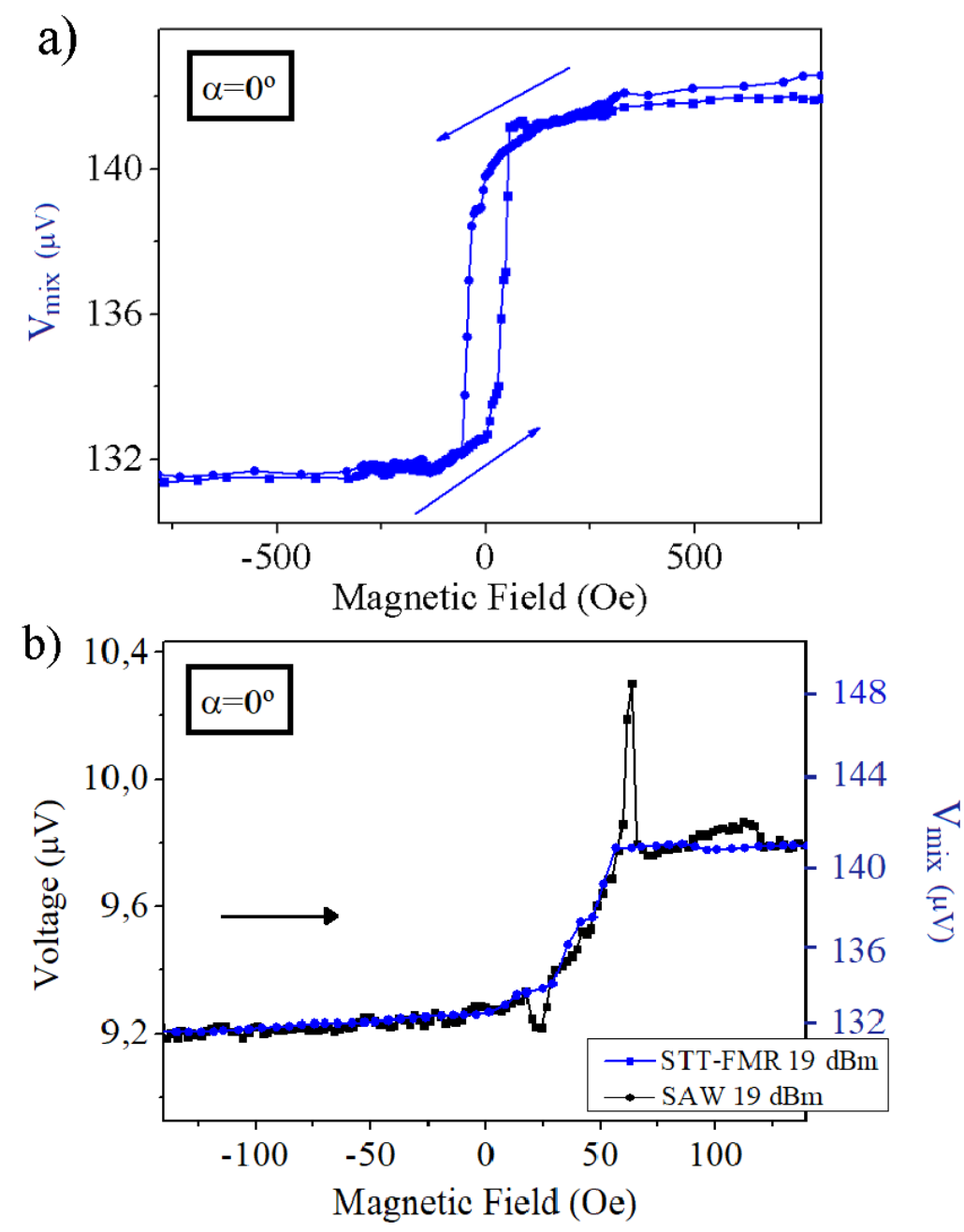

FIG. 105: a) Measurements of $V_{\text {mix }}$ originated by a RF current flowing along the long axis of the FeCoB nanostripe and b) Comparison between $V_{m i x}$ and $V_{D C}$. Magnetic field is applied at $\alpha=0^{\circ}$

We propose that the $V_{D C}$ signal can be partially generated by the inverse spin Hall effect originated by the emission of a spin current from the ferromagnet to the Pt layer on top of the FeCoB. The mechanism generates $V_{D C}$ along the nanostripe triggered by acoustic ferromagnetic resonance of the spins in the magnetic material. This mechanism is, alike non-resonant spin rectification, dependent on the magnetization orientation and therefore occurs when the magnetization reversal takes place. Via magnetoelastic coupling, the SAW excites magnetization $(\vec{M})$ precession. The magnetization precession can relax through the emission of a spin current $\vec{J}_{s}$ into the normal metal $(\mathrm{Pt})$, which acts a capping on top 
of the FeCoB. We detect $\vec{J}_{s}$ along $\mathrm{z}$ via the inverse spin Hall effect, which results in an electric field $\vec{E}_{I S H E} \propto \vec{J}_{s} \times \vec{M}$. More precisely, we measure $V_{D C} \propto \vec{E}_{I S H E} \cdot \vec{x}$. This is depicted in figure 106. The antisymmetric lorentzian does not appear in $V_{\text {mix }}$, therefore we can attribute that feature to the magnetoelastic spin-pumping. From analogue calculations of the photon-driven spin pumping in ferromagnetic-paramagnetic bilayers, we can estimate the resonant precession cone angle $\Theta_{\text {res }}$ of the spins using the scaling law:

$$
\sin ^{2}\left(\Theta_{r e s}\right)=\frac{V_{M S P}}{e \nu P g^{\uparrow \downarrow} \theta_{s h}^{P t} \lambda_{S D} \tanh \left(t_{P t} / 2 \lambda_{S D}\right)}\left(\sigma_{P t} t_{P t}+\sigma_{F e C o B} t_{F e C o B}\right)
$$

where $V_{M S P}=0.45 \mu V$ is the voltage amplitude measured in figure $\left.105 \mathrm{~b}\right), \sigma_{A}$ and $t_{A}$ are the conductivy and the thickness of the A layer respectively, $g^{\uparrow \downarrow}=2 \cdot 10^{19} \mathrm{~m}^{2}$ is the spin mixing conductance, $\nu=1.2 \mathrm{GHz}$ is the frequency of the SAW's, $e$ the elementary charge, $\theta_{s h}^{P t}=0.09$ and $\lambda_{S D}=1.3 \mathrm{~nm}$ are the spin hall angle and the spin diffusion length of the Pt respectively and the ellipticity $P=0.36$ calculated acording to [Mosendz,2010]. This expression can be further simplified in terms of the sample geometry by taking into account that, $\sigma_{P t} t_{P t}+\sigma_{F e C o B} t_{F e C o B}=(R w / L)^{-1}$ where $R=134 \Omega$ is the resistance, $w=2 \mu m$ is the width and $L=6 \mu m$ is the length of the sample. $C \equiv \theta_{s h}^{P t} \lambda_{S D} \tanh \left(t_{P t} / 2 \lambda_{S D}\right)=8.35 \cdot 10^{-11}$ $m$ is a constant at a given temperature. Thus, we can rewrite eq. 25 as:

$$
\sin ^{2}\left(\Theta_{r e s}\right)=\frac{V_{M S P}}{e \nu P C g^{\uparrow \downarrow} R w}
$$

The expression with these data yields a precession cone angle of $\Theta_{\text {res }}=5.3^{\circ}$. We can further calculate the RF virtual driving field by $\mu_{0} h_{M E}=\frac{1}{2} \mu_{0} \Delta H \Theta_{\text {res }}$, with $\Delta H=5 \mathrm{mT}$ as the FWHM of $V_{M S P}$, turns out into a $h_{M E}=230 \mu T$. Equation 26 allows to discern why the acoustically driven spin pumping voltage is not observable in the Ni nanostripe, since with such a small width $(400 \mathrm{~nm})$ and the rest of parameters being similar, the magnetoelastic spin-pumping voltage would be too small to be measured and it is instead masked by the non-resonant spin rectification voltage.

Figure 105 shows that the spin rectification voltage and the acoustically driven spin pumping voltage can overlap as they manifest when the magnetization reversal process takes 


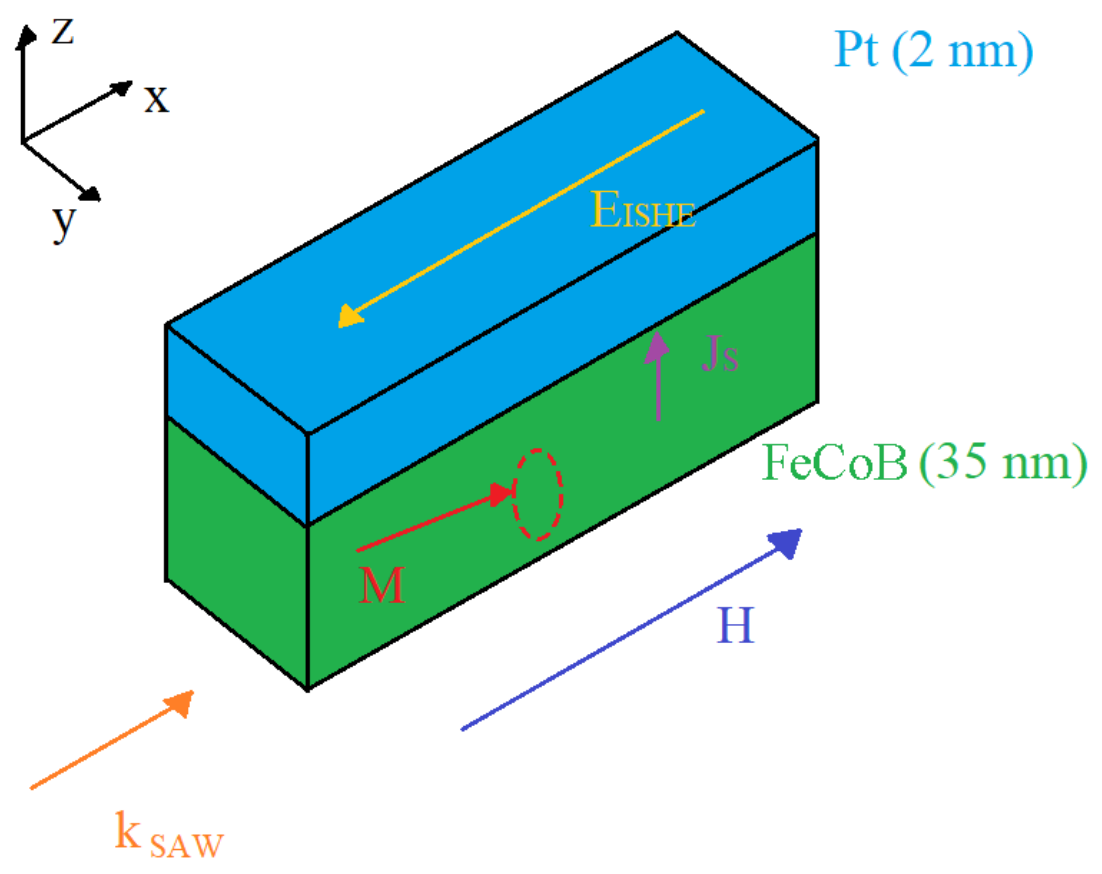

FIG. 106: Schematics of the SAW's driving the resonant $M$ precession that emits a spin current $J_{s}$ into the Pt. $J_{s}$ is detected via inverse spin Hall effect.

place (out of saturation). Since the amplitude of the acoustically driven voltage with this specific geometry is in the range of the tenths of $\mu V$ and and the non resonant rectification voltage can reach tens of $\mu V$, it is probable that the spin rectification contribution masks the magnetoelastic spin-pumping one. However, in this setup with the external magnetic field applied parallel to the long axis of the nanostripe $(\alpha=0)$, the magnetization is mostly parallel to the SAW's propagation direction, which minimizes the contribution of the non resonant spin rectification voltage.

At this point, we perform the measurement of the voltage induced by the SAW's in the FeCoB nanostripe, with the external magnetic field applied perpendicular to the long axis of the nanostripe $\alpha=90^{\circ}$. Measurements for different powers applied to the SAW resonator are presented in figure $107 \mathrm{a}$ ), where, as it was obsered in the case of the Ni nanostripe at $\alpha=90^{\circ}, V_{D C}$ present two different offset voltages at saturation. In figure $107 \mathrm{~b}$ ) it is presented the comparison of the AMR measurement in absence of SAW's and the $V_{D C}$ signal measured with a $\mathrm{H}$ field at $\alpha=90^{\circ}$. It is clear the correlation between both curves, since the $V_{D C}$ signal presents its variation when the magnetization of the FeCoB nanostripe is 
rotating. Which can be observed in the AMR measurement. Along with the 's' type of curve obtained in the measurements, $V_{D C}$ signals present two dips (marked by red arrows), which appear at symmetric fields with respect to $\mathrm{H}=0$.
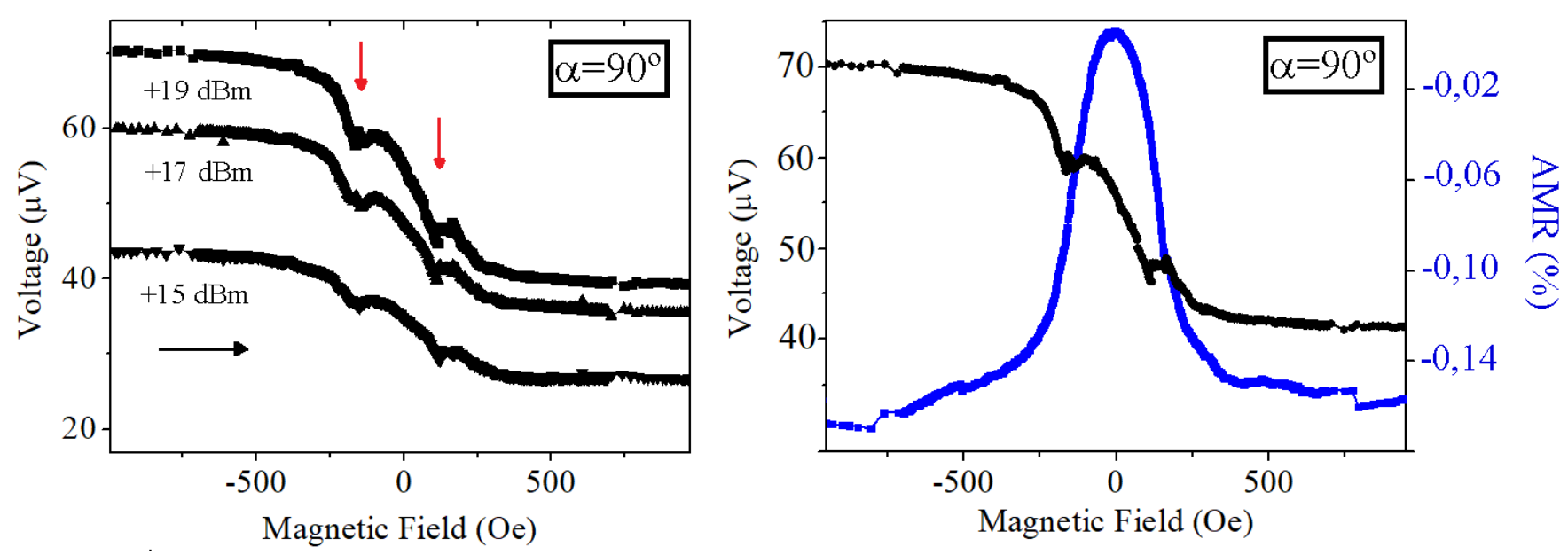

FIG. 107: a) Measurements of the voltage induced by the SAW's $\left(V_{D C}\right)$ performed in the FeCoB nanostripe at $\alpha=90^{\circ}$ for different powers applied to the SAW resonator. b) Comparison of the $V_{D C}$ measurement at $+19 \mathrm{dBm}$ and the AMR in absence of SAW's with the same orientation $\alpha=90^{\circ}$

In figure 108 a) it is presented the AMR in this configuration in presence of SAW's with a RF signal power of $+19 \mathrm{dBm}$ and compared to the voltage $V_{D C}$ induced by the SAW's. It can be observed that the induced voltage is relatively large compared to the resistive AMR signal, which generates a large distortion in the AMR. In figure 108 a) the AMR measurement does not have the original bell-like shape, but instead is more alike to the induced voltage signal, displayed in figure 108 b). It is obvious in this case that in presence of SAW's, the induced voltage is added to the AMR measurements and generates the distorsion. As in the previous cases, we propose that the SAW's are inducing a non resonant spin rectification voltage, which is dependent on the orientation of the magnetization. As the magnetization switchig in the nanostripe is carried out by the rotation out of the longitudinal axis of the spins with an external field applied at $\alpha=90^{\circ}$. In order to confirm the non-resonant spin rectification effect as the origin of the $V_{D C}$ signal, we expose intentionally the FeCoB 
nanostripe to a RF current and measure the mixing voltage that is induced in the nanostripe due to the application of the RF current as it is depicted in figure 101.
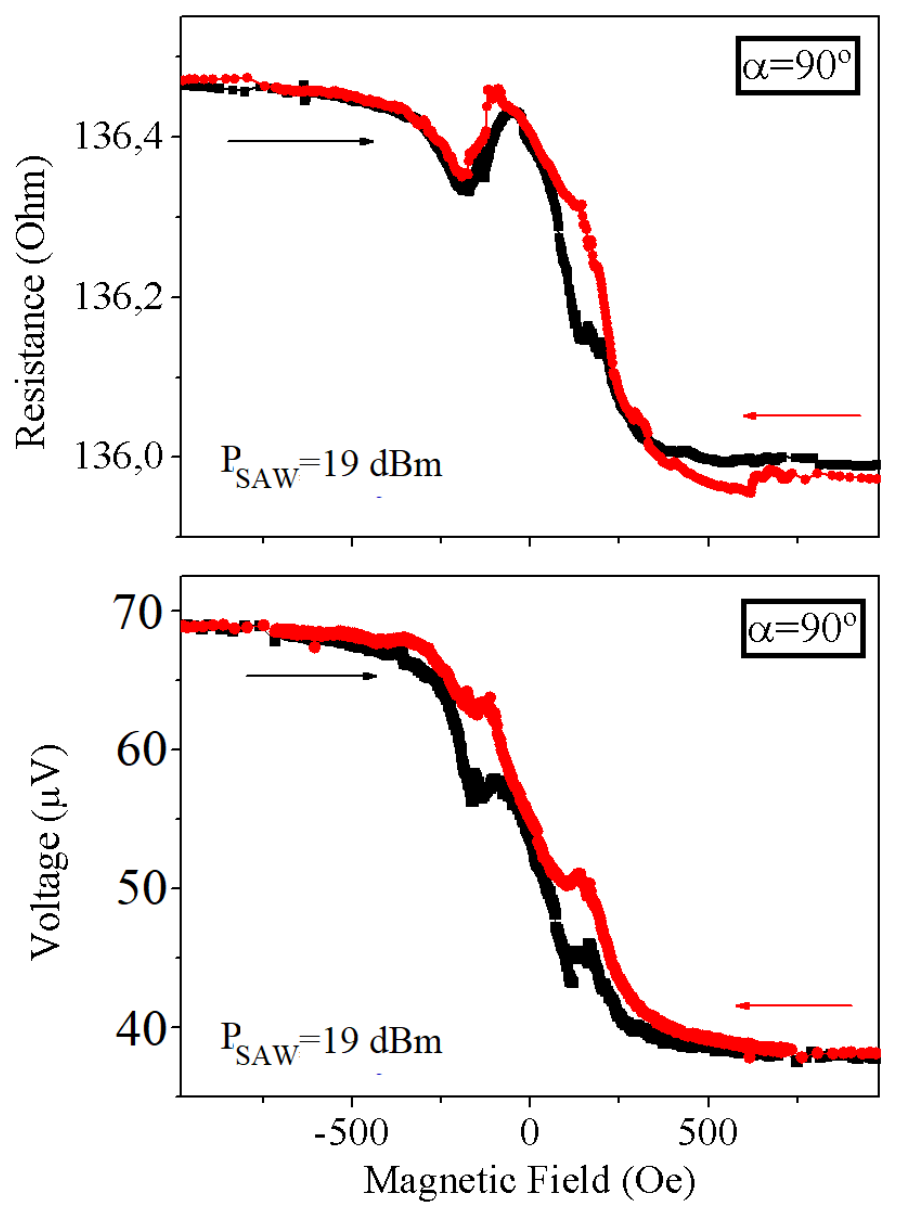

FIG. 108: a) Magnetoresistance measurement for the FeCoB nanostripe with an external magnetic field applied perpendicularly to the long axis. b) $V_{D C}$ Measurement as a function of the external field applied perpendicular to the long axis for a $19 \mathrm{dBm}$ signal power applied to the SAW resonator.

The RF current is applied to flow along the nanostripe and its frequency is set to the fundamental mode of the Rayleigh frequency of the SAW resonator. Measurements are displayed in figure 109, where the voltage induced by the SAW's $V_{D C}$ and the mixing voltage $V_{\text {mix }}$ are compared. The similarities between both curves become clear as both measurements present different offset voltages at saturation, whereas at low fields, where the reversal process occurs $V_{D C}$ and $V_{m i x}$ present their variation. Furthermore, two clear dips are observed 
at fields of \pm 150 Oe, in both curves. These two dips can be related to the ferromagnetic resonance of the sample, since they occur at symmetric fields. This fields are quite low, but it can be consistent since the frequency of the RF current applied is relatively low.

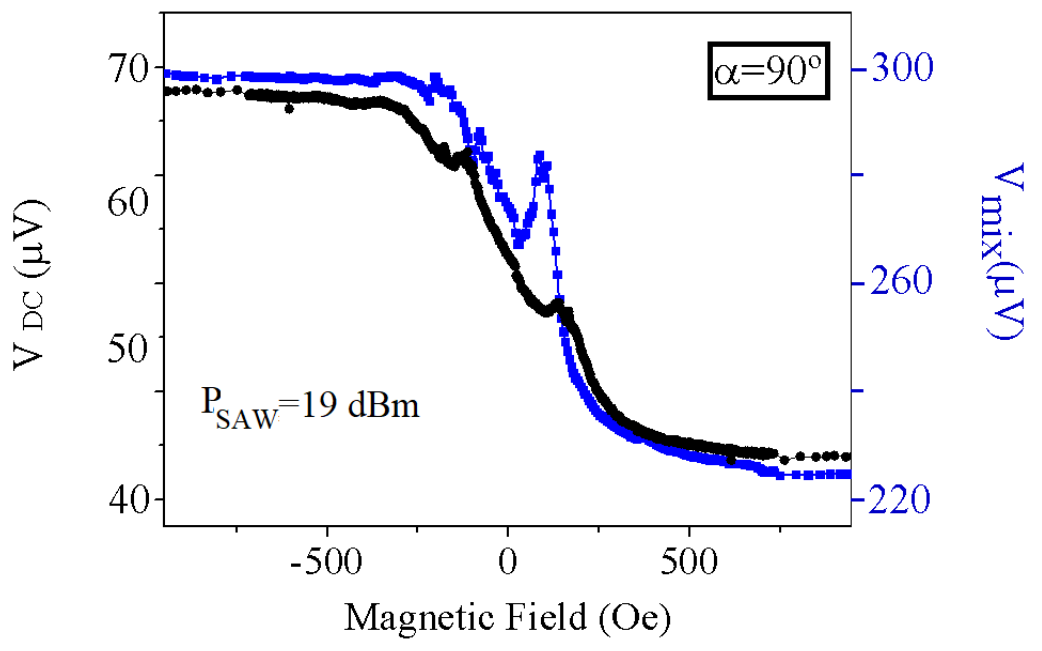

FIG. 109: Comparison of the induced-by-the-SAW's voltage $V_{D C}$ (black curve) and the mixing voltage $\left(V_{m i x}\right)$ generated by the application of a $\mathrm{RF}$ current in a FeCoB nanostripe with an external magnetic field orientation of $\alpha=90^{\circ}$.

\section{E. Conclusions}

The objective of this chapter was to describe the the different voltages that emerge across the long axis of the nanostripes, when they are in presence of surface acoustic waves (SAW's). In this chapter we have distinguish between various mechanisms that give rise to different induced voltages in the nanostripes. The first effect that we associate to an induced voltage is the acoustoelectric effect, which manifests as an offset voltage that is measured in the magnetoresistance measurement of the magnetic nanostripes. This acoustoelectric effect occurs when a semiconductor or a metal film is subject to the action of the SAW's, since the free carriers experience an unidirectional net displacement due to the action of the travelling mechanical and electrical waves. The transfer of linear momentum from the SAW's to the free electrons result in build-up voltage. This is observed firstly in the magnetoresistance measurement of the magnetic nanostripe, however, the electroacoustic effect appear in any 
type of material. We measure directly the voltage induced by the SAW's in a nanostripe made of $\mathrm{Au}$, as a function of an external magnetic field, and we obtained a constant voltage independent of $\mathrm{H}$. This is the expected voltage induced by the electroacoustic effect in every nanostripe.

We also measure directly the voltage $V_{D C}$ induced by the SAW's in the Ni nanostripes. It is observed, once the $V_{D C}$ measurements are carried out, that this voltage is the responsible for the asymmetry in the magnetoresistance measurements measured in presence of SAW's. Furthermore, $V_{D C}$ for different orientations of the external magnetic field present a clear correlation between their features and the AMR measurements, meaning that the $V_{D C}$ present its main variations when the reversal process of the magnetization takes place. In order to understand the origin for this voltage, it is necessary to understand that along with the SAW's, an electrical potential travels in the piezoelectric substrate in addition to the mechanical displacement. This electrical potential can be electrically shorted by the conductive nanostripe resulting in a radiofrequency $R F$ current across the nanostripe. We found that the induced voltage is due to a non resonant spin rectification voltage due to the mixing of the motion of the magnetization in presence of a SAW-induced RF current. In the case of the magnetic nanostripes, this non resonant spin rectification voltage is overlapped with the constant electroacoustic voltage that appears in the nanostripes because its conductive behaviour. Finally the non resonant spin rectification origin is confirmed by the application of a RF current directly to the Ni nanostripes and comparing the mixing voltage generated with the induced-by-the-SAW's voltage. At every orientation they match qualitatively, confirming the origin of the voltage induced by the SAW's.

We have also measured directly the voltage induced by the SAW's $V_{D C}$ in the FeCoB nanostripes and we found again that $V_{D C}$ is the responsible for the asymmetry measured in the magnetoresistance measurements in presence of SAW's. Besides from $V_{D C}$, we measure in this FeCoB nanostripe the voltage that it is originated from the mixing of a RF current with the oscillating magnetization $\left(V_{\text {mix }}\right)$, in order to compare this voltge with the induced-bythe-SAW's voltage and check if they have the same origin. Measurements with the external magnetic field pplied parallel to the long axis of the nanostripe, show that $V_{m i x}$ and $V_{D C}$ show some similiarities, however, $V_{D C}$ present some peaks that can not be related to the mixing 
voltage. Those peaks are instead, related to the acoustically-driven spin pumpin, since the SAW's are capable of forcing the precession of the spins within the ferromagnet and inject a spin current from the ferromagnet into the Pt layer grown on top of the FeCoB layer. That spin current is detected via inverse spin Hall effect, which is the responsible for the final $V_{D C}$ signal that is measured. This contribution as well as the one coming from the non resonant spin rectification effect are overlapped since both occur when the magnetization reversal takes place. We use the comparison between $V_{m i x}$ and $V_{D C}$ to separate each contribution apart. The voltage induced by the SAW's $V_{D C}$, is also measured when the orientation of the external magnetic field is perpendicular to the long axis of the FeCoB nanostripe, however in this case the non-resonant spin rectification voltage is so large that masks completely any voltage due to the acoustically-driven spin pumping. We perform for this FeCoB nanostripe and at $\alpha=90^{\circ}$, measurements of $V_{m i x}$. By comparing $V_{m i x}$ and $V_{D C}$ we conclude that they both have the same origin. Finally, from all the $V_{D C}$ measurements acquired during this chapter, we conclude that the amplitude of the non-resonant spin rectification voltage is dependent on the angle that forms the magnetization with the direction of propagation of the SAW's, being maximum when they are perpendicular and minimum when they are parallel. 


\section{Appendix: Surface Acoustic Waves}

The existence of Rayleigh waves was predicted in 1885 by Lord Rayleigh [Rayleigh, 1885], after whom they were named. He described the behaviour and properties of acoustic waves that propagate on the plane surface of an elastic solid. The particles in Rayleigh waves move in ellipses in planes normal to the surface and parallel to the direction of propagation, the major axis of the ellipse is normal to the surface. At the surface and at shallow depths this motion is retrograde (counterclockwise when the wave travels from left to right). At greater depths the particle motion becomes prograde. In addition, the motion amplitude decays and the eccentricity changes as the depth into the material increases. The depth of significant displacement in the solid is approximately equal to the acoustic wavelength. A visual representation of the Rayleigh wave is displayed in figure 110.

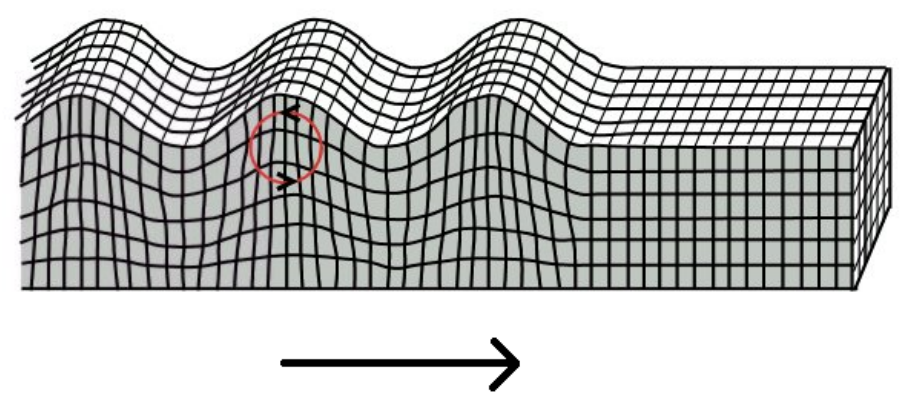

FIG. 110: Picture of a Rayleigh wave. The motion of a surface particle and the propagation direction are depicted [mtu.edu].

In this thesis, the generation of the SAW's that travel towards our magnetic nanostructures is carried out by a SAW resonator. First described in 1965 by [White, 1965], this technique allows the launching and detection of surface acoustic waves. The SAW resonator is a device fabricated on top of a piezoelectric layer characterized by its IDTs, (interdigital transducers) a series of metallic bars alternatively connected to different contact pads. The IDTs convert acoustic waves to electrical signals and vice versa by exploiting the piezoelectric effect. 

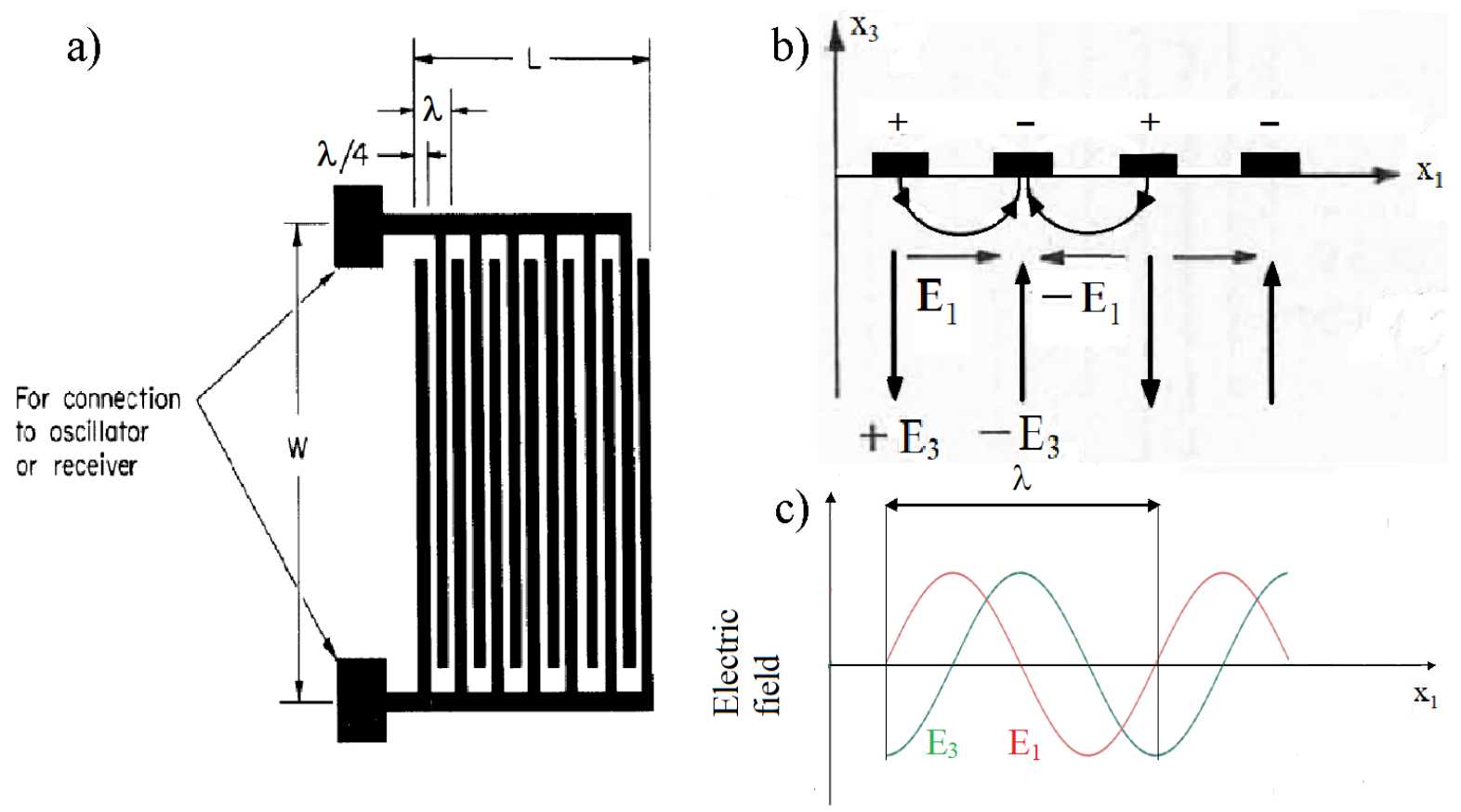

FIG. 111: Electrode pattern of an IDT [White, 1965]. Electric field components generated by the SAW resonator depicted in the spatial coordinates b) and along the propagation direction c)

When a RF potential is applied to an IDT, shown in figure 111 a) it causes a periodic displacement of the surface and also the electric fields components shown in figure $111 \mathrm{~b}$ ) and c) are generated [De Klerk, 1972]. The functioning frequencies of the SAW resonator depend on the design of the IDT (the periodicity length) and the characteristics of the piezoelectric. Any transducer system which is capable of generating the appropriate compressional and shear displacements with a 90 degrees phase relationship will readily launch Rayleigh waves. The reasons for the success of this structure is appreciated when the physical nature of Rayleigh surface waves is understood. The retrograde elliptical particle motion of a Rayleigh wave is due to two components of displacement always present, and 90 degrees out of phase with one another, via a shear displacement normal to the surface and a compressional displacement parallel to the surface. Figure 112 shows the phase relationship between the two displacement. The two wave equations for this type of surface wave are:

$$
\begin{aligned}
& u_{1}=U_{1} \cos k\left(x_{1}-v_{R} t\right) \\
& u_{3}=U_{3} \sin k\left(x_{3}-v_{R} t\right)
\end{aligned}
$$


where $U_{1}$ and $U_{3}$ are respectively the maximum compressional and shear displacements, $k$ is the propagation vector, $v_{R}$ is the Rayleigh wave velocity, $t$ represents time and $x_{1}$ is the distance in the direction of propagation.

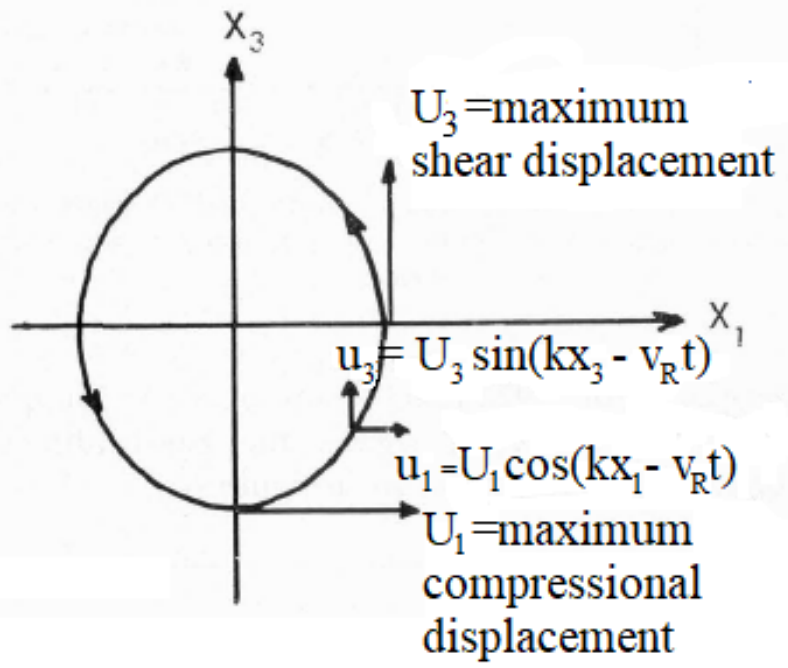

FIG. 112: Displacement components of a Rayleigh wave [De Klerk, 1972]. 


\section{Bibliography}

Aliev, G.N., Goller, B., Snow, P.A., Heinrich, H. and Aigner, R., Porous Silicon Bulk Acoustic Wave Resonator with Integrated Transducer, Nanoscale Research Letters 2012, $7: 378$.

Bai, L., Feng, Z., Hyde, P., Ding, H.F. and Mu, C.-M., Distinguishing Spin Pumping from Spin Rectification in a Pt/Py Bilayer through Angle Dependent Line Shape Analysis, Applied Physics Letters 102, 242402, (2013).

Baibich, M. N., Broto, J.M., Fert, A., Nguyen van Dau, F., Petroff, F., Etienne, P., Creuzet, G., Friedrich, A. and Chazelas, J., Giant Magnetoresistance of (001)Fe/(001)Cr Magnetic Superlattices, Physical Review Letters 61, 2472 - (1988).

Bartoli, F., Aubert, T., Moutaouekkil, M., Streque, J., Pigeat, P., Hage-Ali, S., Boulet, P., M'Jahed, H., Bou Matar, O. and Talbi, A., Theoretical and Experimental Study of ScAlN/Sapphire Structure Based SAW Sensor. IEEE Sensors, (2017).

Bauer, U., Emori, S. and Beach, G.S.D., Voltage-Controlled Domain Wall Traps in Ferromagnetic Nanowires, Nature Nanotechnology 8, 411-416 (2013).

Beach, G.S.D., Nistor, C., Knutson, C., Tsoi, M. and Erksine, J.L., Dynamics of FieldDriven Domain-Wall Propagation in Ferromagnetic Nanowires, Nature Materials 4, 741-744 (2005).

Berk, C., Jaris, M. , Yang, W. , Dhuey, S. , Cabrini S. and Schmidt, H., Strongly Coupled Magnon-Phonon Dynamics in a Single Nanomagnet, Nature Communications 10, 2652 (2019).

Bhatti, S., Ma, C., Xiaoxi Liu, X. and Piramanayagam S. N., Stress-Induced Domain Wall Motion in FeCo-Based Magnetic Microwires for Realization of Energy Harvesting, Advance Electronics Materials (2018), 1800467. 
Binasch, G., Grünberg, P., Saurenbach, F. and Zinn, W., Enhanced Magnetoresistance in Layered Magnetic Structures with Antiferromagnetic Interlayer Exchange, Physical Review B 39, 4828(R), (1989).

Biswas, A.K., Bandyopadhyay, S. and Atulasimha, J., Acoustically Assisted SpinTransfer-Torque Switching of Nanomagnets: An Energy-efficient Hybrid Writing Scheme for Nonvolatile Memory, Applied Physics Letter 103, 232401 (2013).

Bryan, M.T., Dean, J. and Allwood, D.A., Dynamics of Stress-Induced Domain Wall Motion, Physical Review B 85, 144411 (2012).

Büyükköse, S., Hernández-Mínguez, A., Vratzov, B., Somaschini, C., Geelhaar, L., Riechert, H., van der Wiel, W. G. and Santos, P. V., High-Frequency Acoustic Charge Transport in GaAs Nanowires. Nanotechnology 25, 135204 (2014).

Carignan, L-P., Lacroix, C., Ouimet, A., Ciureanu, M., Yelon, A. and Ménard, A., Magnetic Anisotropy in Arrays of $\mathrm{Ni}, \mathrm{CoFeB}$, and Ni/Cu Nanowires, Journal of Applied Physics 102, $023905(2007)$

Chen, M., Chien, C-L. Searson, P.C., Potential Modulated Multilayer Deposition of Multisegment $\mathrm{Cu} / \mathrm{Ni}$ Nanowires with Tunable Magnetic Properties, Chemistry of Materials, 18, 6, 1595-1601, (2006).

Chen, M. and Searson, P.C., Micromagnetic Behavior of Electrodeposited Ni/Cu Multilayer Nanowires, Journal of Applied Physics 93, 8253 (2003).

Collins, J.H., Lakin, K.M., Quate, C.F. and Shaw, H.J., Amplification of Acoustic Surface Waves with Adjacent Semiconductor and Piezoelectric Crystals. Applied Physics Letters 13, 314 (1968).

Davis, S., Baruth, A. and Adenwalla, S., Magnetization Dynamics Triggered by Surface 
Acoustic Waves, Applied Physics Letters 97, 232507 (2010).

De Klerk, J., Past, Present and Future of Surface Elastic Waves, Journal de Physique Colloques, (1972), 33 (C6), 182-188.

Dean, J., Bryan, M. T., Cooper, J. D., Virbule, A., Cunningham, J. E. and Hayward, T. J., A sound Idea: Manipulating Domain Walls in Magnetic Nanowires Using Surface Acoustic Waves, Applied Physics Letters 107, 142405 (2015).

Díaz, J., Quirós, C., Álvarez-Prado, L.M., Aroca, C., Ranchal, R., Ruffoni, R. and Pascarelli, S., Determination of the magnetostrictive atomic environments in FeCoB alloys, Physical Review B 85, 134437 - (2012).

Dreher, L., Weiler, M., Pernpeintner, M., Huebl, H., Gross, R., Brandt, M.S. and Goennenwein, S.T.B., Surface acoustic wave driven ferromagnetic resonance in nickel thin films: Theory and experiment, Physical Review B 86, 134415 (2012)

Drovosekov, A.B., Kreines, N.M., Savitsky, A.O., Kravtsov, E.A., Ryabukhina, M.V., Proglyado, V.V. and Ustinov, V.V., Magnetization and Ferromagnetic Resonance in Fe/Gd Multilayer: Experiment and Modelling, Journal of Physics: Condensed Matter, 29, 11, (2017).

Drovosekov, A.B., Savitsky, A.O., Kholin, D.I., Kreines, N.M., Proglyado, V.V., Ryabukhina, M.V., Kravtsov, E.A. and Ustinov, V.V., Twisted Magnetization States and Inhomogeneous Resonance Modes in a Fe/Gd Ferrimagnetic Multilayer, Journal of Magnetism and Magnetic Materials, 475, 668 (2019).

Edrington, W., Singh, U., Dominguez, M. A., Alexander, J. R., Nepal, R. and Adenwalla. S., SAW Assisted Domain Wall Motion in Co/Pt Multilayers, Applied Physics Letters 112, $052402(2018)$.

Finizio, S., Foerster, M., Buzzi, M., Krüger, B., Jourdan, M., Vaz, C. A. F., Hockel, J., 
Miyawaki, T., Tkach, A., Valencia, S., Kronast, F., Carman, G.P., Nolting, F. and Kläui, M., Magnetic Anisotropy Engineering in Thin Film Ni Nanostructures by Magnetoelastic Coupling, Physical Review Applied 1, 021001 (2014).

Foerster, M., Macia F., Statuto, N., Finizio, S., Hernández-Mínguez, A., Lendínez S., Santos, P., Fontcuberta, J., Hernández, J.M., Kläui, M. and Aballe, L., Direct Imaging of Delayed Magneto-Dynamic Modes Induced by Surface Acoustic Waves, Nature Communications 8, 407 (2017).

Grollier, J., Chanthbouala, A., Matsumoto, R., Anane, A., Cros, V., Nguyen van Dau, F. and Fert, A., Magnetic Domain Wall Motion by Spin Transfer, Comptes Rendus Physique 12 (2011) 309-317.

Grollier, J., Cros, V. and Fert, A., Synchronization of Spin-Transfer Oscillators Driven by Stimulated Microwave Currents, Physical Review B 73, 060409(R) - (2006).

Groves, T.R., Electron Beam Lithography, The Art of Fabricating Nanoelectronic and Nanophotonic Devices and Systems (2014), 80-115.

Halilov, S.V., Perlov A. Y., Oppeneer, P.M., Yaresko, A.N. and Antonov, V.N., Magnetocrystalline anisotropy energy in cubic Fe, Co, and Ni: Applicability of local-spin-density theory reexamined, Physical Review B 57, 16 (1998)

Hertel, R. and Kirschner, J., Magnetization Reversal Dynamics in Nickel Nanowires, Physica B 343 206-210, (2004).

Herzer, G., Grain Structure and Magnetism of Nanocrystalline Ferromagnets. IEEE Transactions on Magnetics, 25, 5 (1989).

Ho, P., Zhang, J., Bono, D. and Chen, J., Oersted Field and Spin Current Effects on Magnetic Domains in [Co/Pd]15 Nanowires, IEEE Transactions on Magnetics 52(6), (2016). 
Hong, K. and Giordano, N., Resistance of a Domain Wall in a Thin Ferromagnetic Wire, Journal of Physics: Condensed Matter 10, 401 (1998).

Hosoito, N., Hashizume, H., Ishimatsu, N., Bae, I.-T., Srajer, G., Lang, C., Ventakaraman, C. and Nelson, C., Magnetic Structures of an Iron-Gadolinium Multilayer at Low Temperatures, Japanese Journal of Applied Physics, 41, 1331 (2002).

Hsu, P-J., Kubetzka, A., Finco, A., Romming, N., von Bergmann, K. and Wiesendanger, R., Electric-Field-Driven Switching of Individual Magnetic Skyrmions, Nature Nanotechnology 12, 123-126 (2017).

Huai, Y., Albert, F., Nguyen, P., Pakala, M. and Valet, T., Observation of Spin-Transfer Switching in Deep Submicron-Sized and Low Resistance Magnetic Tunnel Junctions, Applied Physics Letters 84, 3118 (2004).

Huai, Y., Spin-Transfer Torque MRAM (STT-MRAM): Challenges and Prospects, AAPPS Bulletin (2008), 18, 6 .

Insepov, Z., Emelin, E., Kononenko, O., Roshuchupkin, D.V., Tnyshtykbayev, K.B. and Baigarin, K.A., Surface Acoustic Wave Amplification by Direct Current-Voltage Supplied to Graphene Film. Applied Physics Letters 106023505 (2015).

Kamohara, T., Akiyama, M. and Kuwano, N., Influence of Polar Distribution on Piezoelectric Response of Aluminum Nitride Thin Films, Applied Physics Letters 92, 093506 (2008).

Kawada, T., Kawaguchi, M. and Hayashi, M., Unidirectional planar Hall voltages induced by surface acoustic waves in ferromagnetic thin films. Physical Review B 99, 184435 (2019).

Kozlov, S. N., Skryabina, O. V., Egorov, S. V., Golovchanskiy, I. A., Klimenko, A. A., Napolskii, K. S. and Stolyarov, V. S., Magnetoresistance of a Single Polycrystalline Nickel Nanowire. Journal of Applied Physics 125, 063902 (2019). 
Laur, V., Queffelec, P., Rasoanoavy, F., Lebedev, G., Viala, B. and Thi, M.P., Microwave Magnetoelectric Couplings in FeCoB/Piezoelectric Bilayers, IEEE Transactions on Magnetics 49,3 ,(2013)

Lee, E.W. and Asgar, M.A., The Magnetostriction of Nickel, Proceedings of the Royal Society of London. Series A, Mathematical and PhysicalSciences,326, 1564 (1971), 73-85

Lee, D., Lee, N., Choi, G. and Hee Cho, H., Heat Transfer Characteristics of a Focused Surface Acoustic Wave (F-SAW) Device for Interfacial Droplet Jetting, Inventions 2018, $3(2), 38$.

Lepadatu, S., Mihai, A.P., Claydon, J.S., Maccherozzi, F., Dhessi, S.S., Kinane, C.J., Langridge, S. and Marrows, C.H., The Increase of the Spin-Transfer Torque Threshold Current density in Coupled Vortex Domain Walls, Journal of Physics: Condensed Matter, 24 (2012) 024210.

Li, W., Buford, B., Jander, A. and Dhagat, P., Magnetic Recording with Acoustic Waves, Physica B 448 (2014) 151-154.

Li, W., Buford, B., Jander, A. and Dhagat, P., Acoustically Assisted Magnetic Recording: A New Paradigm in Magnetic Data Storage, IEEE Transactions on Magnetics, 50, 3, (2014).

Liu, L., Moriyama, T., Ralph, D.C. and Burham R.A., Spin-Torque Ferromagnetic Resonance Induced by the Spin Hall Effect, Physical Review Letters, 106, 036601, (2011).

Löffel, J.F., Meier, J.P., Doudin, B., Asermet, J-P. and Wegner, W., Random and Exchange Anisotropy in Consolidated Nanostructured Fe and Ni: Role of Grain Size and Trace Oxides on the Magnetic Properties, Physical Review B 57, 5, (1998).

Mancoff, F.B., Rizzo, N.D., Engel, B.N. and Tehrani, S., Phase-locking in Double-PointContact Spin-Transfer Devices, Nature 437, 393-395, (2005). 
Martin, F., Muralt, P., Dubois, M-A. and Pezous, A., Thickness Dependence of the Properties of Highly c-Axis Textured AlN Thin Films, Journal of Vacuum Science \& Technology A 22, 361 (2004).

Matei, E., Enculescu, I., Toimil-Morales, M-E. and Leca, A., Magnetic Configurations of Ni-Cu Alloy Nanowires Obtained by the Template Method, Journal of Nanoparticle Research, 15:1863, (2013).

McGuire, T. and Potter, R., Aniostropic Magnetoresistance in ferromagnetic 3d Alloys IEEE Transactions on Magnetics 11, 1018 (1975).

Mellnik, A.R., Lee, J.S., Richardella, A., Grab, J.L., Mintum, P.J., Fischer, M.H., Vaezi, A., Manchon, A., Kim, E-A., Samarth, N. and Ralph, D.C., Spin Transfer Torque Generated by the Topological Insulator $\mathrm{Bi}_{2} \mathrm{Se}_{3}$, Nature 511, 449-451 (2014).

Milyutin, E., Harada, S., Martin, D., Carlin, J.F., Grandjean, N., Savu, V., VasquezMena, O., Brugger, J. and Muralt, P., Sputtering of (001) AlN Thin Films: Control of Polarity by a Seed Layer, Journal of Vacuum Science \& Technology 28, 61 (2010).

Montoya, S.A., Couture, S., Chess, J.J., Lee, J.C.T., Kent, N., Im, M-Y., Kevan, S.D., Fischer, P., McMorran, B.J., Roy, S., Lomakin, V. and Fullerton, E.E., Resonant properties of dipole skyrmions in amorphous Fe/Gd multilayers, Physical Review B 95, 224405 - (2017)

Muñoz, M. and Prieto, J.L., Supression of the Intrinsic Stochastic Pinning of Domain Walls in Magnetic Nanostripes, Nature Communications, 2, 562 (2011).

Naik, R.S., Lutsky, J.J., Reif, R., Sodini, C.G., Becker, A., Fetter, L., Huggins, H., Miller, R., Pastalan, J., Rittenhouse, G. and Wong, Y.H., Measurements of the Bulk, C-Axis Electromechanical Coupling Constant as a Function of A1N Film Quality, IEEE Transactions on Ultrasonics, Ferroelectrics, and Frequency Control, 47, 1, (2000). 
Neu, V., Biele R., Singh, A. and Schultz, L., Modeling of Intergrain Exchange Coupling for Quantitative Predictions of $\delta m$ Plots, IEEE Transactions on Magnetics, 46, 6 (2010).

Pai, C-F., Liu, L., Li, Y., Tseng, H.W., Ralph, D.C. and Buhrman, R.A., Spin Transfer Torque Devices Utilizing the Giant Spin Hall Effect of Tungsten, Applied Physics Letters 101, 122404 (2012).

Parkes, D. E., Cavill, S. A., Hindmarch, A. T., Wadley, P., McGee, F., Staddon, C. R., Edmonds, K. W., Campion, R. P., Gallagher, B. L. and Rushforth, A. W., Non-Volatile Voltage Control of Magnetization and Magnetic Domain Walls in Magnetostrictive Epitaxial Thin Films. Applied Physics Letters 101, 072402 (2012).

Parkin, S.S.P., Hayashi, M. and Thomas, L., Magnetic Domain-Wall Racetrack Memory, Science 320, 190 (2008).

Partin, G.S., Vas'kovskii, V.O., Svalov, A.V., Eremin, E.V., Panova, M.A. and Vasil'ev, V.N, Magnetic Resonance in Multilayer Gd/Si/Co Magnetic Films, Journal of Experimental and Theoretical Physics, 102, 1, 131 (2006).

Pimpin, A. and Srituravanich, W., Review on Micro- and Nanolithography Techniques and their Applications, Engineering Journal, 16 Issue 1, ISSN 0125-8281, (2012).

Pfeiffer, H.C., Recent Advances in Electron-Beam Lithography for the High-Volume Production of VLSI Devices, IEEE Transactions on Electric Devices, 26, 4, (1979).

Prieto, J. L., Aroca, C., López, E., Sánchez, M. C. and Sánchez, P., Reducing Hysteresis in Magnetostrictive-Piezoelectric Magnetic Sensors. IEEE Transactions on Magnetism 34, 3913 (1998).

Qin, G. W. and Zuo L. , Tailoring Exchange Coupling between Magnetic Nano-Grains of High Density Magnetic Recording Media, Materials Science Forum, 638-642, 2944-2949, (2010). 
Ralph, D.C. and Stiles, M.D., Spin Transfer Torques, Journal of Magnetism and Magnetic Materials 320 (2008) 1190-1216.

Rippard, W.H., Pufall, M.R., Kaka, S., Silva, T.J., Russek, S.E. and Katine, J.A., Injection Locking and Phase Control of Spin Transfer Nano-oscillators, Physics Review Letters 95, $067203-(2005)$.

Romming, N., Hanneken, C., Menzel, M., Bickel, J.E., Wolter, B., von Bergmann, K., Kubetzka, A. and Wiesendanger, R., Writing and Deleting Single Magnetic Skyrmions, Science 341, 636 (2013).

Roy, K., Ultra-Low-Energy Straintronics Using Multiferroic Composites, Proceedings of SPIE - The International Society for Optical Engineering 03(02):91670U (2014).

Sampath, V., D’Souza, N., Bhattacharya, D., Atkinson, G.M., Bandyopadhyay, S. and Atulasimha, J., Acoustic-Wave-Induced Magnetization Switching of Magnetostrictive Nanomagnets from Single-Domain to Nonvolatile Vortex States, Nano Letters (2016), 16, 9, 5681-5687.

Sankey, J.C., Cui, Y-T., Sun, J.Z., Sloczewski, J.C., Burharm, R.A. and Ralph, D.C., Measurement of the spin-transfer-torque vector in magnetic tunnel junctions, Nature Physics 4,67-71 (2008).

Sanz-Hervás, A., Clement, M., Iborra, E., Vergara, L., Olivares, J. and Sangrador, J., Degradation of the Piezoelectric Response of Sputtered -Axis AlN Thin Films with Traces of non-(0002) x-Ray Diffraction Peaks, Applied Physics Letters 88, 161915 (2006).

Stiles, M.D. and Zangwill, A. Anatomy of Spin-Transfer Torque, Physical Review B 66, 014407 - (2002).

Susano, M., Proenca, M-P, Moraes, S., Sousa, C-T. and Araujo, J-P., Tuning the Mag- 
netic Properties of Multisegmented Ni/Cu Electrodeposited Nanowires with Controllable Ni Lengths, Nanotechnology, 19, 27(33):335301, (2016).

Suzuki, M., Yanagitani, T. and Odagawa, H., Polarity-Inverted ScAlN Film Growth by Ion Beam Irradiation and Application to Overton Acoustic Wave (000-1)/(0001) Film Resonators, Applied Physics Letters 104, 172905 (2014).

Tang, G., Han, T., Teshigahara, A., Iwaki, T. and Hashimoto, K.-Y., Enhancement of Electromechanical Coupling Factor by Mass Loading in Layered SAW Device Structures, IEEE, Frequency Control Symposium and the European Frequency and Time Forum, 416419, (2015).

Tejada, J., Chudnovsky, E. M., Zarzuela, R., Statuto, N., Calvo-de la Rosa, J., Santos, P. V. and Hernández-Mínguez, A., Switching of magnetic moments of nanoparticles by surface acoustic waves. Euro Physics Letters 118, 37005 (2017).

Teshigahara, A., Hashimoto K. and Akiyama, M., Scandium Aluminum Nitride : Highly Piezoelectric Thin Film for RF SAW Devices in multi GHz Range. (2012) IEEE International Ultrasonics Symposium.

Thevenard, L., Gourdon, C., Prieur, J-Y, von Bardeleben, H.J., Vincent, S., Becerra, L, Largeau L. and Duquesne J-Y, Surface-Acoustic-Wave-Driven Ferromagnetic Resonance in $(\mathrm{Ga}, \mathrm{Mn})(\mathrm{As}, \mathrm{P})$ Epilayers, Physical Review B 90, 094401 - (2014).

Thevenard, L., Camara, I., Prieur, J-Y, Rovillain, P., Lemaitre, A., Gourdon, C. and Duquesne J-Y, Strong Reduction of the Coercivity by a Surface Acoustic Wave in an Outof-Plane Magnetized Epilayer, Physical Review B 93, 140405(R) (2016).

Thevenard, L., Camara, I. S., Majrab, S., Bernard, M., Rovillain, P., Lemaitre, A., Gourdon, C. and Duquesne, J.-Y., Precessional Magnetization Switching by a Surface Acoustic Wave, Physical Review B. 93, 134430 (2016). 
Weiler, M., Dreher, L., Heeg, C., Huebl, H., Gross, R., Brandt, M. S. and Goennenwein1, S. T. B. Elastically Driven Ferromagnetic Resonance in Nickel Thin Films, Physical Review Letter 106, 117601 (2011).

Weiler, M., Huebl, H., F. S. Goerg, F.S., Czeschka, F.D., Gross, R. and Goennenwein, S. T. B. Spin Pumping with Coherent ElasticWaves, Physical Review Letter 108, 176601 $(2012)$

White, D.L., Amplification of Ultrasonic Waves in Piezoelectric Semiconductors. Journal of Applied Physics 33, 2547 (1962).

White, R. M. and Voltmer, F. W., Direct Piezoelectric Coupling to Surface Elastic Waves. Applied Physics Letters 7, 314 (1965).

Wu, T., Bur, A., Wong, K., Zhao, P., Lynch, C. S., Amiri, P.K., Wang K. L. and Carman, G. P., Electrical Control of Reversible and Permanent Magnetization Reorientation for Magnetoelectric Memory Devices. Applied Physics Letters 98, 262504 (2011).

Yang, W. G. , Jaris, M., Hibbard-Lubow,D. L., Berk, C. and Schmidt, H., Magnetoelastic Excitation of Single Nanomagnets for Optical Measurement of Intrinsic Gilbert Damping, Physical Review B. 97, 224410 (2018).

Yoshida, K. and Yamanishi, M., Interaction Between Surface Elastic Waves and Drifting Carriers in Layered System. Japan Journal of Applied Physics 7 (1968) 1143-1144.

Zeng, Z., Finocchio, G. and Jiang, H., Spin Transfer Nano-Oscillators, Nanoscale, (2013), 5, 2219-2231.

Zhu, X.F., Harder, M., Tayler, J., Wirthmann, A., Zhang, B., Lu, W., Gui, Y.S. and Hu, C.-M., Non Resonant Spin Rectification in the Absence of an External Applied Magnetic Field, Physical Review B, 83, 140402 (R), (2011). 
Zhukov, A., Blanco J.M., Ipatov, M., Chizhik A. and Zhukov, V., Manipulation of Domain Wall Dynamics in Amorphous Microwires Through the Magnetoelastic Anisotropy, Nanoscale Research Letters (2012), 7:223. 